\title{
3-D Modeling of Coronal Mass Ejections with STEREO/SECCHI Data
}

\author{
Dissertation \\ zur Erlangung des mathematisch-naturwissenschaftlichen Doktorgrades \\ "Doctor rerum naturalium" \\ der Georg-August-Universität Göttingen \\ im Promotionsprogramm ProPhys \\ der Georg-August University School of Science (GAUSS)
}

vorgelegt von

Eckhard Bosman

aus Köln

Göttingen, 2016 


\section{Betreuungsausschuss:}

Prof. Dr. Stefan Dreizler

Sonnenphysik und Stellare Astrophysik, Institut für Astrophysik, Georg-August-Universität Göttingen, Germany

Prof. Dr. Ansgar Reiners

Sonnenphysik und Stellare Astrophysik, Institut für Astrophysik, Georg-August-Universität Göttingen, Germany

Dr. Volker Bothmer

Sonnenphysik und Stellare Astrophysik, Institut für Astrophysik, Georg-August-Universität Göttingen, Germany

\section{Mitglieder der Prüfungskommission:}

Prof. Dr. Ansgar Reiners

Sonnenphysik und Stellare Astrophysik, Institut für Astrophysik, Georg-August-Universität Göttingen, Germany

Prof. Dr. Jörg Büchner

Sonne und Heliosphäre, Max-Planck-Institut für Sonnensystemforschung, Göttingen, Germany

\section{Weitere Mitglieder der Prüfungskommission:}

Dr. Volker Bothmer

Sonnenphysik und Stellare Astrophysik, Institut für Astrophysik, Georg-August-Universität Göttingen, Germany

Prof. Dr. Michael Seibt

Halbleiterphysik, 4. Physikalisches Institut, Georg-August-Universität Göttingen, Germany

Prof. Dr. Andreas Tilgner

Geophysikalische Fluiddynamik, Institut für Geophysik, Georg-August-Universität Göttingen, Germany

Prof. Dr. Manuela Temmer

Physik der Sonne und der Heliosphäre, Institut für Physik, Karl-Franzens-Universität Graz, Österreich 




\section{Contents}

1 Introduction 1

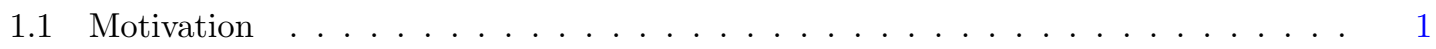

1.2 Scientific Objectives . . . . . . . . . . . . . . . . . . 2

2 The Sun $\quad 5$

2.1 The Structure of the Sun . . . . . . . . . . . . . . . . . 5

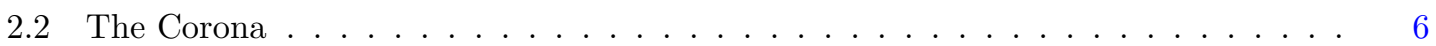

2.3 Coronal Mass Ejections . . . . . . . . . . . . . . . . . . . 8

2.3.1 CME Characteristics and Observational Features . . . . . . . . . . . 8

2.3.2 Models for Coronal Mass Ejections . . . . . . . . . . . . . . . . . . . . 9

2.3 .3 The Origination of CMEs . . . . . . . . . . . . . . . . . 9

2.3.4 The 'Cremades and Bothmer CME Scheme' . . . . . . . . . . . . . 11

2.4 Solar Activity . . . . . . . . . . . . . . . . . . . . . . . . . . . 13

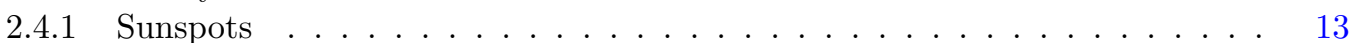

2.4 .2 Prominence Eruptions . . . . . . . . . . . . . . . . . . . . . . . . . . . . . . . . . . . . . . . 14

2.4 .3 Active Regions . . . . . . . . . . . . . . . . . . . . . . . 14

2.4 Post Eruptive Arcades . . . . . . . . . . . . . . . . . . . . 16

2.4 .5 Flares . . . . . . . . . . . . . . . . . . 16

3 Instrumentation and Data $\quad 19$

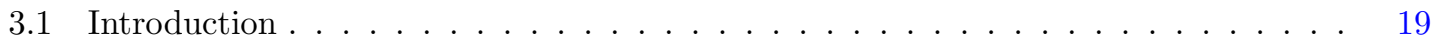

3.2 The STEREO Mission . . . . . . . . . . . . . . . . . . . . . . . . . . . . . . . . . . . . . . . . . . . . . . . . . .

3.3 SECCHI Instruments . . . . . . . . . . . . . . . . . . . . . . . . . . . . . . . . . . . . . . . . . . . . . . . . . . . .

3.3.1 The Coronagraph - COR2 . . . . . . . . . . . . . . . . . 24

3.3.2 The Extreme UltraViolet Imager - EUVI . . . . . . . . . . . . . . . . . . 26

4 Morphological Classification of CMEs $\quad 29$

4.1 Description of CME Classes . . . . . . . . . . . . . . . . . . . . . . . . . . . . . 29

4.2 Comparison with CME Survey by Howard (1985) . . . . . . . . . . . . . . . . 33

4.2 .1 Introduction . . . . . . . . . . . . . . . . . . . . 33

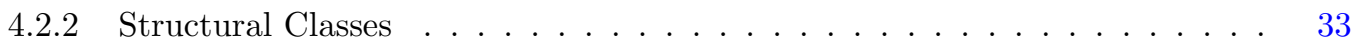

4.2.3 Results from Comparison . . . . . . . . . . . . . . . . 38

5 The Graduated Cylindrical Shell Model $\quad 41$

5.1 Overview of CME Reconstruction Techniques . . . . . . . . . . . . . . . . . . . 41

5.2 The GCS Model . . . . . . . . . . . . . . . . . . . . . . . . . . . . . . . . . . . . . . . . . . . . . . . . . .

5.2.1 Geometry of the GCS Model . . . . . . . . . . . . . . . . 42

5.2.2 Electron Density Distribution . . . . . . . . . . . . . . . . 43

5.2.3 Ray-Tracing Code ... . . . . . . . . . . . . . . . . . . 44

5.3 STEREO/SECCHI Data Acquisition and Data Processing . . . . . . . . . . . . 45

5.3 .1 STEREO/SECCHI Data Provision . . . . . . . . . . . . . . . 46

5.3 .2 Software Requirements and Installation . . . . . . . . . . . . . . . . . . . . . . . . .

5.3 .3 Data Usage for Modeling . . . . . . . . . . . . . . . . . . . . 47

5.4 CME Modeling with the GCS Technique . . . . . . . . . . . . . . . . . 48

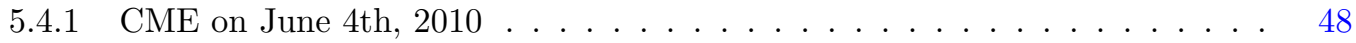

5.4 .2 CME on November 16 th, $2007 \ldots \ldots \ldots$

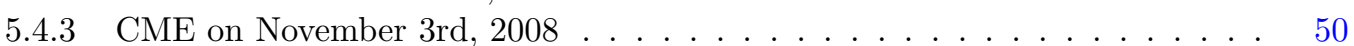

5.4 CME on February 2nd, $2011 \ldots \ldots \ldots \ldots$

5.4.5 CME on April 26th, $2008 \ldots \ldots \ldots$

5.4 .6 CME on March 12th, 2011 . . . . . . . . . . . . . . . . . . . 55

5.4 Sources of Error using GCS . . . . . . . . . . . . . . . . . . . . 55

5.4 The Changing Spacecraft Positions . . . . . . . . . . . . . . . 57

5.5 Identification of the CME's Source Region . . . . . . . . . . . . . . . . . . 63 
6 Selected CME Classes and the Flux Rope Model 65

6.1 CME Class 01 - Halo CME . . . . . . . . . . . . . . . . . . . . . 65

6.2 CME Class 06 - 'Fish'-CME . . . . . . . . . . . . . . . . . . . 65

6.3 CME Class 07 - Classical CME . . . . . . . . . . . . . . . . . . . . . 68

6.4 CME Class 09 - 'Laurel Wreath' CME . . . . . . . . . . . . . . . . . . . . . 69

6.5 Comments and Comparison to the Morphology Study by Vourlidas (2013) . . . . 70

7 Statistical Results from Geometrical Fitting and Parameter Analysis 73

7.1 Introduction . . . . . . . . . . . . . . . . . . . . . . 73

7.1.1 The Overall CME List . . . . . . . . . . . . . . . . . . . . . 73

7.1 .2 The 'Best-of' CME List . . . . . . . . . . . . . . . . . . . . 74

7.1.3 The GCS Modeling Results List . . . . . . . . . . . . . . . . . . . 74

7.2 GCS Modeling Results . . . . . . . . . . . . . . . . . . . . . . . 75

7.2.1 Carrington Longitude . . . . . . . . . . . . . . . . . . 75

7.2 .2 Heliospheric Latitude . . . . . . . . . . . . . . . . . . . . . . 75

7.2 .3 Tilt Angle . . . . . . . . . . . . . . . . . 77

7.2 .4 Aspect Ratio . . . . . . . . . . . . . . . . . . 78

7.2 .5 Half Angle . . . . . . . . . . . . . . . . . . . . . 79

7.2 .6 Height .......................... 81

7.2 .7 GCS Flux Rope Diameter . . . . . . . . . . . . . . . . . 82

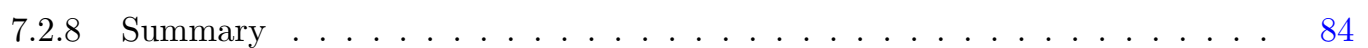

7.3 GCS Modeling Results and CME Classes . . . . . . . . . . . . . . . . 85

7.3.1 GCS Aspect Ratio of CME Classes . . . . . . . . . . . . . . . . . 85

7.3.2 GCS Half Angle of CME Classes . . . . . . . . . . . . . . . . . . 86

7.3.3 GCS Latitude of CME Classes . . . . . . . . . . . . . . . . . 87

7.3 .4 Summary ............................. 88

7.4 Applications for the GCS Modeling . . . . . . . . . . . . . . . . 89

8 Summary and Outlook $\quad 91$

8.1 Scientific Questions and Answers . . . . . . . . . . . . . . . . . . . . 91

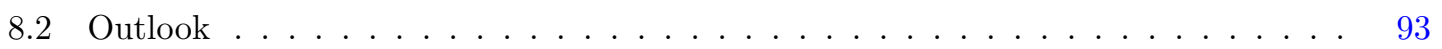

$\begin{array}{lr}\text { A Appendix } & 95\end{array}$

A.1 The IDL Code for Data Processing . . . . . . . . . . . . . . . . . . . . 95

A.2 Selected CME Classes and the Flux Rope Model . . . . . . . . . . . . . . . . . . 97

A.2.1 CME Class 02 - Diffuse CME . . . . . . . . . . . . . . . . . . . . 97

A.2.2 CME Class 03 - Partial Halo CME . . . . . . . . . . . . . . . . . . . . 98

A.2.3 CME Class 04 - 'Ring' type CME . . . . . . . . . . . . . . . . . . 99

A.2.4 CME Class 05 - 'Jet-like' CME . . . . . . . . . . . . . . . . . . . 100

A.2.5 CME Class 08 - Distorted CME . . . . . . . . . . . . . . . . . . 101

A.2.6 CME Class 10 - Non-rated CME . . . . . . . . . . . . . . . . . . . 102

A.3 GCS Aspect Ratio . . . . . . . . . . . . . . . . . . . . . . . . . . . 102

A.4 The 'Best-of' CME List . . . . . . . . . . . . . . . . . . . . . . . . . . . . . . . . . 104

A.5 The GCS Modeling Results List . . . . . . . . . . . . . . . . . . . . . . . . . 108

A.6 The CME Online Database . . . . . . . . . . . . . . . . . . . . . . . . . . . 112

A.7 Acronyms . . . . . . . . . . . . . . . . . . . . . 114

$\begin{array}{lr}\text { B References } & 115\end{array}$ 


\section{Abstract}

This thesis reports on the three-dimensional analysis of coronal mass ejections (CMEs) in order to answer questions about their morphology and to derive their three-dimensional geometry. The questions are about the detailed 3-D structure, orientation and position of CMEs which are observed near the Sun $\left(10-20 r_{\odot}\right)$ with coronagraphs of the STEREO mission.

The Sun as main actor for space weather and in particular as source of CMEs raises many exciting unanswered questions. Some of these about the CME's geometry are treated in this thesis. Getting a better understanding of CMEs is very helpful to improve forecasts of Earth directed CMEs for shielding humans and their infrastructure from the sometimes harmful space weather effects which are caused by CMEs.

Within the scope of this work a study was started for the 3-D analysis of CMEs based on the coronagraph observations of the STEREO twin satellites. Both spacecraft observe the Sun and inner heliosphere from Earth-like orbits, with STEREO-A moving ahead and STEREO-B trailing behind Earth. The coronagraph observations are analysed for the time period starting at the beginning of the STEREO observations in January 2007 until December 2011 when the solar activity increased. 1071 CMEs were identified in the STEREO/SECCHI/COR2 coronagraph observations and summarised in an overall CME list. From this list a 'Best-of' CME list with 264 events was extracted based on the visual appearance of the CME's white-light structure in the coronagraph images. These CME events are analysed in this thesis in detail.

During the inspection of the COR2 coronagraph images it was noticeable that the CMEs occur with different morphologies in their two dimensional white-light appearance. Because several shapes of CME appearances with certain patterns occurred often, ten CME classes are defined and introduced. Subsequently, the 'Best-of' CMEs are categorised according to these CME class definitions. The different CME morphologies which were found during the first inspections of the coronagraph data are investigated in order to find out if CMEs of different white-light appearances can be described as flux ropes with the Graduated Cylindrical Shell (GCS) model. In the second step it is examined how these CMEs look like in terms of this model. It is found that the different appearances and morphologies of CMEs observed with coronagraphs can indeed be fitted with the GCS modelling technique resulting in a 3-D flux rope geometry. The synthetic coronagraph images which are generated from Thomson scattering calculations confirm their 3-D flux rope geometry. Since the CMEs can be described with the Graduated Cylindrical Shell (GCS) model, their 3-D geometry, position and orientation is analysed and discussed in the second part of this thesis. For this purpose the 3-D GCS modeling technique is used to compute the 3-D geometry with STEREO/SECCHI/COR2 coronagraph data for 241 CMEs.

The results from the 3-D GCS CME analysis show that during the phase of low solar activity (January 2007 - January 2010) small-scale CMEs at low latitudes dominate. This is proved by low values of the GCS model parameters aspect ratio $(\kappa \leq 0.4)$ and half angle $\left(\alpha \leq 20^{\circ}\right)$ which describe the spatial expansion of a CME. In contrast, during the second time period with increasing solar activity (February 2010 - December 2011) also large-scale CMEs are observed and CMEs occur also at higher latitudes up to $\pm 60^{\circ}$. The spatial expansion of those CMEs is characterised by a GCS half angle $\alpha>20^{\circ}$ and an aspect ratio $\kappa>0.4$. The analysis of the diameter for GCS modelled CMEs reveals that CMEs observed between 10 and $20 \mathrm{r}_{\odot}$ exhibit a flux rope diameter ranging from 2 to $8 \mathrm{r}_{\odot}$. It is found from GCS modeling that the CMEs are best suitable for fitting within a solar distance of 11 to $17 \mathrm{r}_{\odot}$ when they appear largely expanded and most bright and clear in structure in the COR2 coronagraph's field of view. In comparison to other previous CME studies this one provides a detailed 3-D parametrisation and analysis of a large set of 241 CMEs instead of plane-of-sky measurements which are affected by projection effects. 



\section{Introduction}

Coronal Mass Ejections (CMEs) are eruptions of large plasma clouds, which were discovered in the solar corona. Since hundreds of years the corona is observed during solar eclipses by astronomers. Drawings of the solar corona observed during the solar eclipse in Spain 1860 show already at that time conspicuous coronal structures (Eddy, 1974). Figure 1 presents a drawing with a conspicuous structure in the south-west (bottom right) of the solar corona. It is assumed that this drawing could show a CME.

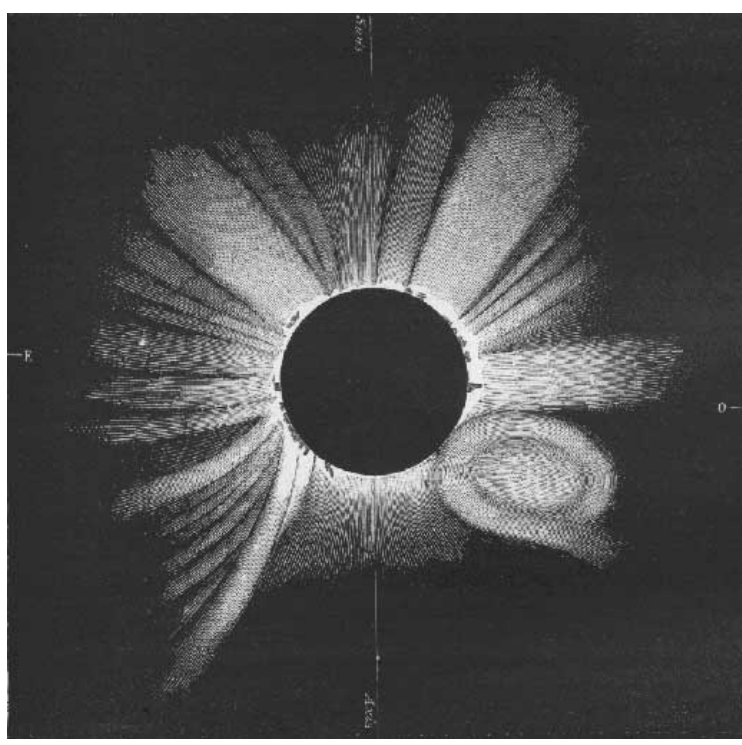

Fig. 1: Drawing of the solar corona, observed during the solar eclipse in 1860, Spain (Eddy, 1974).

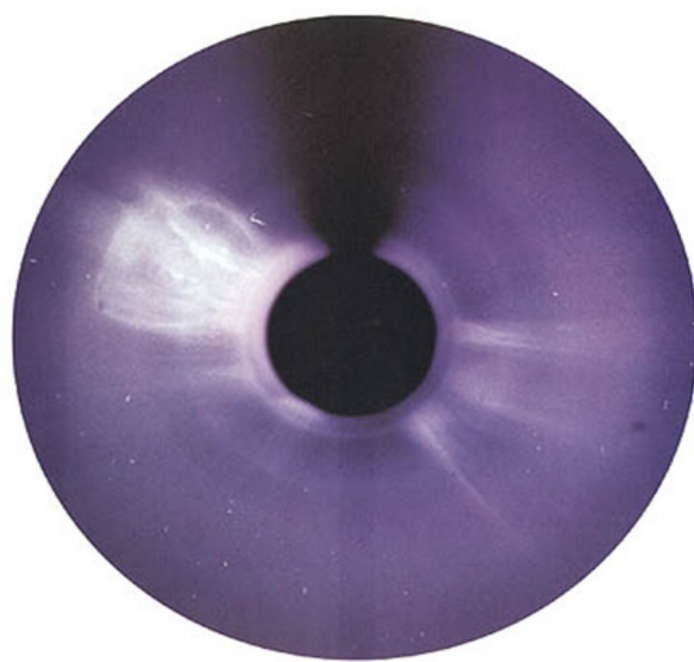

Fig. 2: A CME observation with the coronagraph onboard the 7th Orbiting Solar Observatory mission in the seventies. From OSO-7, NASA http: //history.nasa.gov.

With the advent of satellite-based observations of the Sun in the seventies it was possible to observe the solar corona with space-based coronagraphs. An observation of the corona with the coronagraph of the 7th Orbiting Solar Observatory (OSO-7) mission is shown in Figure 2. This is seen as one of the first observations of a CME. In the following decades coronal mass ejections were continuously examined with more improved satellite-based coronagraphs. Starting with the 7th Orbiting Solar Observatory mission from 1971 - 1973 CMEs were observed thereafter with a film detector on-board the NASA's Skylab (1973 - 1974). During the longer lasting Solwind (1979 - 1985) and Solar Maximum Mission (SMM: 1980, 1984 - 1989) CME observations could be performed over several years. With the launch of the Solar and Heliospheric Observatory (SOHO) in 1995 CMEs are observed to these days with the telescopes of the LASCO (Large Angle and Spectrometric Coronagraph) instrument suite (Howard, 2006).

The white-light appearance of CMEs was investigated with all of these coronagraph observations. The morphology of CMEs was discussed in different ways, for instance as bulbs, loops, clouds or streamer blow-outs (Munro et al., 1979; Wagner, 1984). Models for the CME's structure were introduced like the three-part structure from Illing and Hundhausen (1985) or the flux rope model for CMEs (Chen and Garren, 1993; Rust and Kumar, 1994).

\subsection{Motivation}

Some questions about the CMEs' structure, especially their 3-D structure, could not completely be clarified with the observations of the previous missions. In order to investigate CMEs and their structure the Solar Terrestrial Relations Observatory (STEREO) was developed for this and other scientific objectives. This mission started in October 2006 with two spacecraft. Since mission start 
both satellites observe the Sun and its corona from Earth-like orbits. One spacecraft (STEREOA) is moving ahead of Earth at an orbit of $0.96 \mathrm{AU}$ and the other one (STEREO-B) is trailing behind Earth at 1.04 AU. These trajectories result in an increasing separation angle between both spacecraft. This allows for the first time to watch a CME synchronously from two viewing points in space with the twin observatories and their coronagraphs. For this purpose both spacecraft are almost identically equipped with two coronagraphs which observe the corona with a field of view from 1.5 to $15 \mathrm{r}_{\odot}$. A CME observed with STEREO-A in October 2010 is shown in Figure 3.

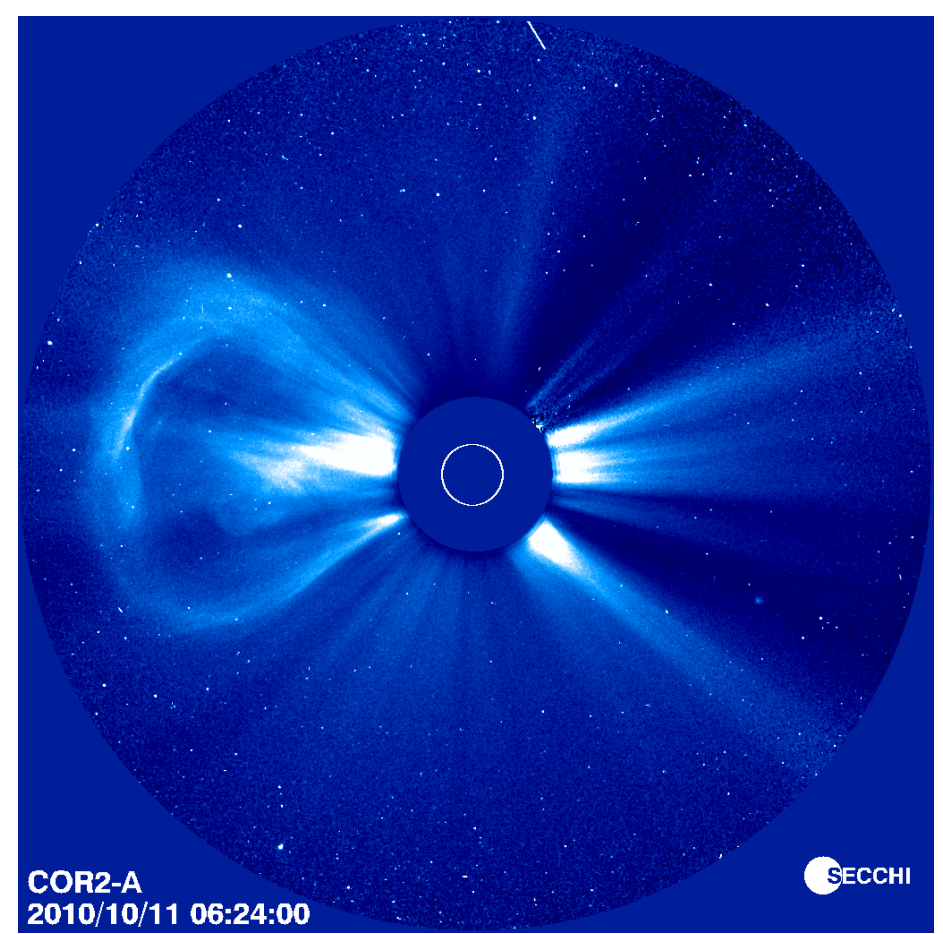

Fig. 3: The white-light appearance of a CME which was observed on October 11th, 2010 with the coronagraph COR2-A of the STEREO-A spacecraft. From http: //secchi.nrl.navy.mil/sccimages STEREO/SECCHI Consortium and NRL.

Coronagraph images which show stereoscopic observations of a CME from both STEREO spacecraft are presented in Chapter 5.4. The STEREO coronagraph observations allow scientists for the first time to examine the CME's white-light appearance from stereoscopic coronagraph images and to deduce the CME's 3-D geometry in agreement with state-of-the-art CME models. The position, orientation, spatial expansion and direction of propagation can be determined quantitatively for CMEs observed near the Sun (2 - 15 solar radii). Also the velocity of CMEs can be calculated by applying the analysis techniques to time series of CME observation.

CMEs are known as the main driver for space weather and its conditions in the interplanetary space and at Earth. An Earth-directed CME with a high velocity and strong magnetic field can cause large disturbances of the Earth's magnetosphere and ionosphere. This can trigger a geomagnetic storm and increased electric currents in the magnetosphere and ionosphere. Technical system are affected by these space weather conditions. This can lead to disruptions of radio communication and damage of satellite systems as well as an increased radiation exposure for aviation and manned space flight. The study of CMEs can help to improve space weather forecasts and to mitigate these effects.

\subsection{Scientific Objectives}

This thesis presents a CME study with an investigation of the 3-D structure for 241 CMEs observed between January 2007 and December 2011 with the STEREO coronagraphs. Therefore the CMEs' white-light appearances from the coronagraph observations are studied. Different morphologies are found in these observations and subsequently the CMEs are grouped in newly introduced morphology classes. The CME's white-light appearance is examined with a state-of-the-art CME model. 
This so called Graduated Cylindrical Shell (GCS) model (Thernisien et al., 2006) describes the CME geometry with a flux rope structure (Chen and Garren, 1993; Rust and Kumar, 1994). The GCS model is fitted to the CME's white-light appearances from the stereoscopic observations in order to clarify if the CMEs can be described as flux ropes in agreement with this model. The resulting 3-D geometry from GCS modeling is analysed in terms of the CME's position, orientation and spatial expansion. In this context the following questions are discussed:

1. To which extent do the observer positions of the STEREO spacecraft influence the 3-D GCS modeling of CMEs?

2. Which types of CMEs allow a description with the GCS model? What are the difficulties?

3. How does the GCS model look like for a flux rope CME? What are typical 3-D properties?

4. The different classes of CME morphologies and their 3-D properties:

Which characteristics in terms of GCS CME parameters does a CME classification reflect?

5. What are the applications for 3-D GCS modeling of a CME?

The next chapter provides an introduction to the Sun and its corona and to solar activities like prominence eruptions. The theory about CMEs and models which describe the CMEs' characteristics are presented in Chapter 2.3. Afterwards the STEREO mission and spacecraft are described with their coronagraphs which provide the scientific data used to investigate the CMEs. Chapter 4 focuses on the inspection of the CME's white-light appearances and their morphological classification. The examination of these morphological CME classes is presented after an introduction to the GCS model (Chapter 6). The obtained results from the GCS analysis of 241 CMEs, their examination and interpretation are discussed in the subsequent chapter. This work concludes with a short summary about the main results. The introduced scientific questions are taken up in the last chapter and short answers based on the key results of this thesis are given. Finally an outlook on further interesting outstanding research is presented. 



\section{The Sun}

This chapter presents some fundamental aspects of the Sun, in particular its structure, atmosphere and solar activity for a better understanding of the following thesis chapters.

\subsection{The Structure of the Sun}

Our Sun is a star of spectral type G2V and has an absolute magnitude of 4.8. In the last decades several ground based and satellite based observations have revealed more and more detailed information about the Sun. Some characteristics of the Sun are listed below:

\begin{tabular}{ll}
\hline Characteristic & Sun \\
\hline Age & $4.5 \times 10^{9} \mathrm{yr}$ \\
Mass & $1.99 \times 10^{30} \mathrm{~kg}$ \\
Radius & $696000 \mathrm{~km}$ \\
Mean density & $1.4 \times 10^{3} \mathrm{~kg} / \mathrm{m}^{3}$ \\
Mean distance from earth & $1 \mathrm{AU}=150 \times 10^{6} \mathrm{~km}=215 \mathrm{r}_{\odot}$ \\
Radiation emitted (luminosity) & $3.86 \times 10^{26} \mathrm{~W}$ \\
Equatorial rotation period & 26 days \\
Effective blackbody temperature & $5778 \mathrm{~K}$ \\
Composition & $\approx 90 \% \mathrm{H}, 9.9 \% \mathrm{He}, 0.1 \%$ \\
& other elements $(\mathrm{C}, \mathrm{N}, \mathrm{O} \ldots)$ \\
\hline
\end{tabular}

Tab. 1: Some characteristics of the Sun (Kivelson et al., 1995).

The Sun is a gas ball consisting approximately of $90 \%$ hydrogen, $9.9 \%$ of helium and of other elements like carbon, nitrogen and oxygen $(\leq 0.1 \%)$. All the gas is held together and compressed with its own gravitational force. The plasma, generated at high temperatures in the solar interior, extends over several layers of the Sun up to its surface and beyond to the solar atmosphere (Kivelson et al., 1995).

Beginning with the center of the Sun the core extends to $0.25 \mathrm{r}_{\odot}$. There the energy of the Sun is produced by fusion of hydrogen into helium at a temperature of $1.5 \times 10^{7} \mathrm{~K}$ and a density of $1.6 \times 10^{5} \mathrm{~kg} / \mathrm{m}^{3}$.

The produced energy propagates outwards and enters the radiative zone which surrounds the core and extends to $0.75 \mathrm{r}_{\odot}$. In this zone the radiation spreads outside very slow because this region is very opaque and the radiation is innumerable times absorbed and emitted. For comparison, the neutrinos produced in the fusion reactions reach the solar surface at $1 \mathrm{r}_{\odot}$ in only $2 \mathrm{~s}$ but the radiation requires about 10 million years.

The radiative zone is enclosed by the convection zone which ends at $1 \mathrm{r}_{\odot}$ and is affected by differential rotation. In this region the energy is transported by convection. This type of movement results in the formation of granulation visible on the photosphere (Kivelson et al., 1995). A schematic view of the Sun's structure is shown in Figure 4.

The photosphere, at $1 \mathrm{r}_{\odot}$, is defined as the surface of the Sun. The grainy solar surface consists of granules which can be understood as the top of convective cells underneath the solar surface. In general, the appearance of the Sun's atmosphere depends on the wavelength used for the observation and different wavelengths reveal features at different depths in the Sun's atmosphere. The $500 \mathrm{~km}$ thick photosphere exhibits a temperature of $5778 \mathrm{~K}$ and a particle density of $10^{23} \mathrm{~m}^{-3}$ and is the lowest one of all three layers of the solar atmosphere. Most of the Sun's light comes from the photosphere and in white-light images one can see dark sunspots localised mostly in two belts, one in the northern and another in the southern solar hemisphere. In this connection the sunspots position in latitude depends on the solar activity so that with proceeding solar cycle sunspots occur at lower latitudes as described by the Joy's Law and the so-called butterfly diagram (Hale et al., 1919). 


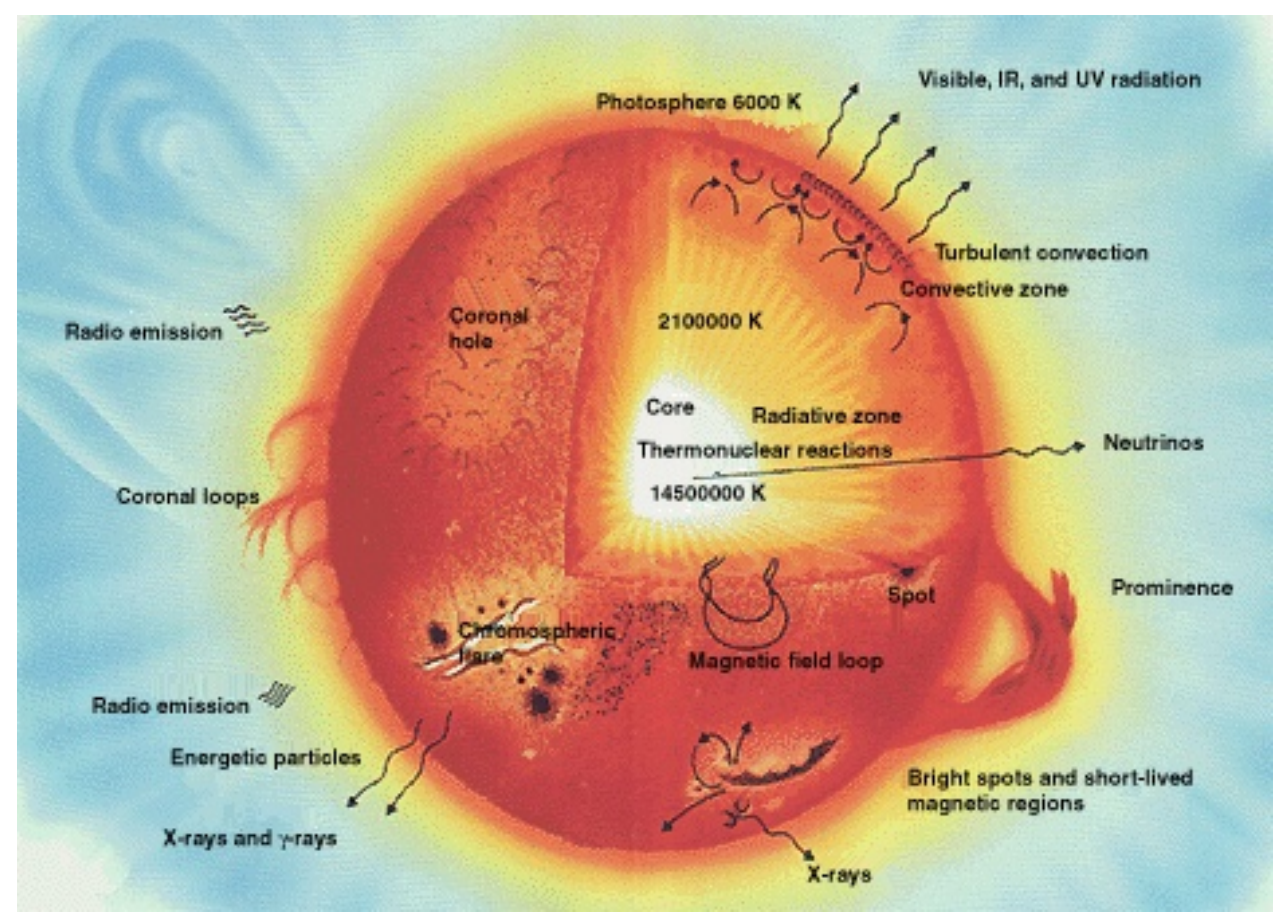

Fig. 4: Schematic structure of the Sun. From High Energy Astrophysics Science Archive Research Center, NASA/GSFC, https://heasarc.gsfc.nasa.gov/.

The chromosphere which extends up to $2000 \mathrm{~km}$ above the photosphere is characterised by emission lines. One of the most intense is the $\mathrm{H} \alpha$ line at $656 \mathrm{~nm}$. For this reason the chromosphere can be well observed with a $\mathrm{H} \alpha$ telescope showing the chromosphere's typical reddish colour. The temperature in this part of the solar atmosphere increases from 4400 to $20000 \mathrm{~K}$ and rapidly increases up to $10^{6} \mathrm{~K}$ in the adjacent transition region which separates the chromosphere from the corona.

The 1 million degree hot corona composes the outer atmosphere layer with a particle density of $10^{15} \mathrm{~m}^{-3}$, for comparison the density near Earth orbit amounts to $10^{7} \mathrm{~m}^{-3}$. From Earth the corona can be only observed during a solar eclipse because the coronal brightness is with a factor of $10^{-6}$ less brighter as the light from the photosphere. For permanent observation of the corona one can use coronagraphs such as these on the SOHO or STEREO spacecraft. The basic characteristic of a coronagraph is an occulter which blocks the direct bright sunlight of the solar disk (Kivelson et al., 1995). The advantage of satellite based in contrast to ground based coronagraphs is that former are not affected by Rayleigh scattering of the sunlight in the upper atmosphere. In addition satellite based telescopes allow a continuous observation of the Sun independent of the Earth's rotation. The STEREO spacecraft and their coronagraphs are described in more detail in Chapter 3.

\subsection{The Corona}

The faint white-light structure of the corona is observable from ground only during solar eclipses. From those observations the white-light of the solar corona is segmented spectroscopicly by their origination in three types. The light of the $\mathrm{F}$ corona is scattered by dust particles and includes the Fraunhofer absorption lines. At larger distances from Sun this light, visible in the ecliptic, is called zodiacal light. The $\mathrm{K}$ corona exhibits a continuous spectrum from the photospheric light which is scattered by free electrons (Thomson scattering) of the coronal plasma. In addition to the continuous spectrum the E corona is composed of the emission in spectral lines caused by the transitions of high-temperature coronal ions like Fe XV (Aschwanden, 2006). The light of 
these emission lines can be used for solar disk observations at extreme ultraviolet wavelengths with dedicated Extreme Ultraviolet Imager on-board STEREO spacecraft (see Chapter 3.3), (Kaiser et al., 2008). The intensities of the mentioned corona types decreases with distance to the solar center as illustrated in Figure 5.

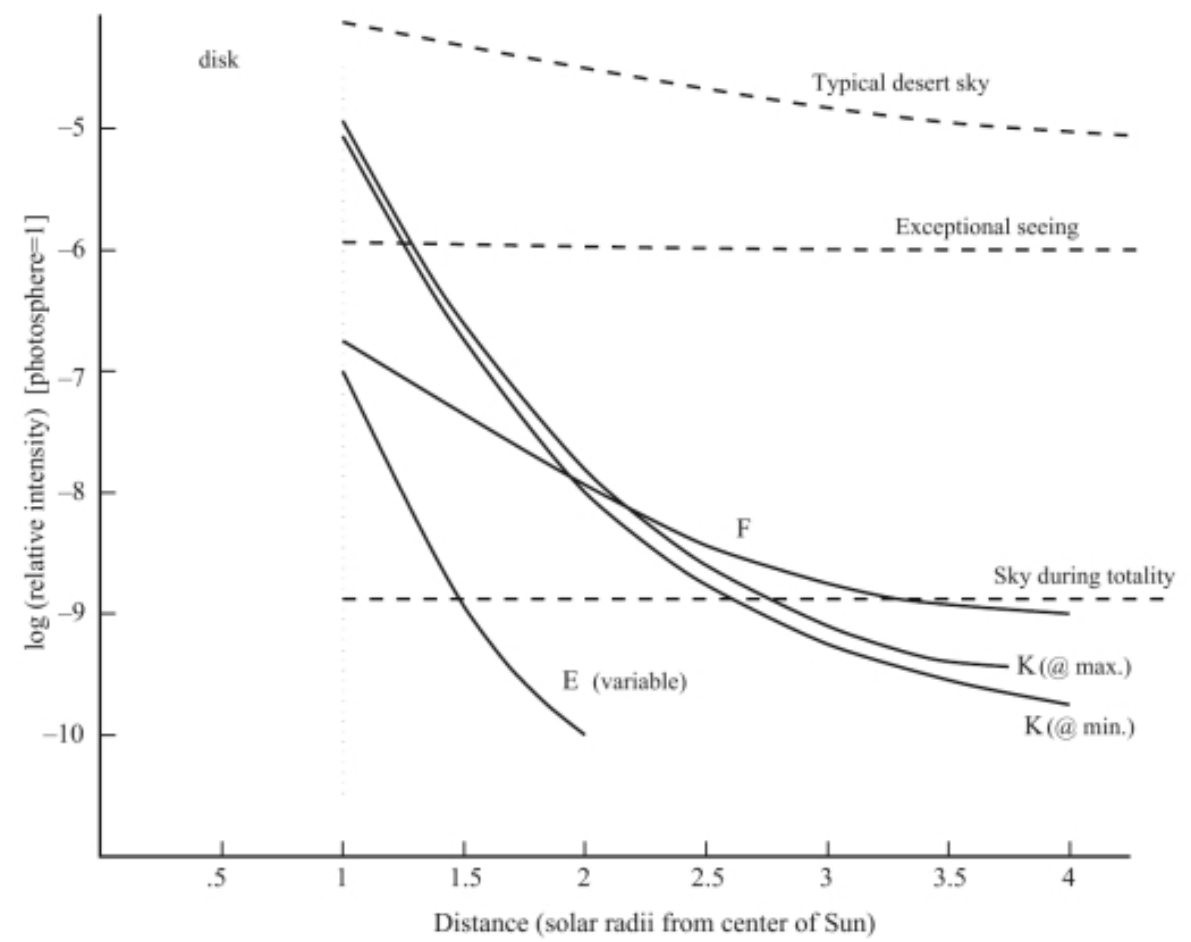

Fig. 5: The relative intensities of the E, F and $\mathrm{K}$ corona (at solar minimum and maximum) plotted as function of the solar distance. From Golub and Pasachoff (2010).

The Sun's corona is structured by the solar magnetic field. This becomes clear if one compares the appearance of the coronal structure at the minimum and maximum of solar activity as illustrated in Figure 6. There the corona is pictured visible during the solar minimum in 1994 and during the

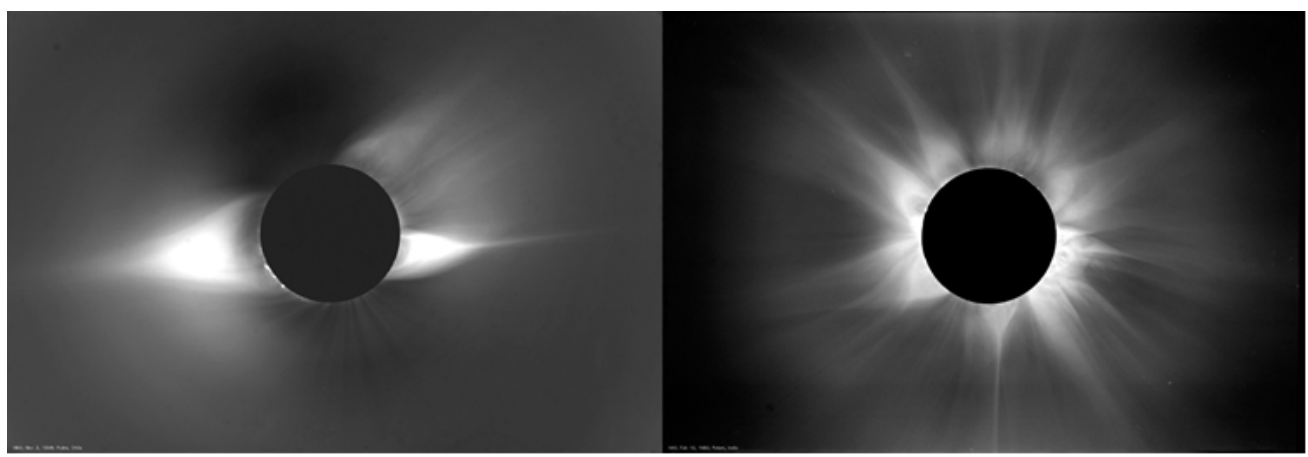

Fig. 6: The typical shape of the solar corona expanded around the solar equator is shown on the left. This observation was made in 1994 during the solar activity minimum of solar cycle 22 . In contrast the corona observed at the maximum of solar activity in 1980 is presented on the right. In this case the coronal structure is characterised by several helmet streamers occurring over all latitudes. From High Altitude Observatory, Boulder, CO, USA.

solar maximum in 1980 (Stix, 2002). 


\subsection{Coronal Mass Ejections}

Coronal Mass Ejections (CMEs) were first observed from space in the 1970's with the coronagraphs on-board the OSO 7 and the Skylab missions as published by Neupert (1988) and Gosling et al. (1974). In the course of time CMEs could be observed more accurately with increasing optical resolution. Based on these observations the white-light appearance of coronal mass ejections was described in different ways as erupting bubbles, bulbs (Crifo et al., 1983), coronal loops (Gosling et al., 1974), flux tubes (Mouschovias and Poland, 1978), or flux ropes (Chen and Garren, 1993; Rust and Kumar, 1994). A definition of CMEs was formulated by Hundhausen et al. (1984) which describes what can be understood as a coronal mass ejection observed in coronagraph images.

\section{Definition of a CME as:}

" [...] an observable change in coronal structure that occurs on a time scale between a few minutes to hours and involves the appearance of a new, discrete, bright, white-light feature in the coronagraph field of view."

Hundhausen et al. (1984)

Figure 7 shows an example of such a CME observed with the SOHO/LASCO/C2 coronagraph.

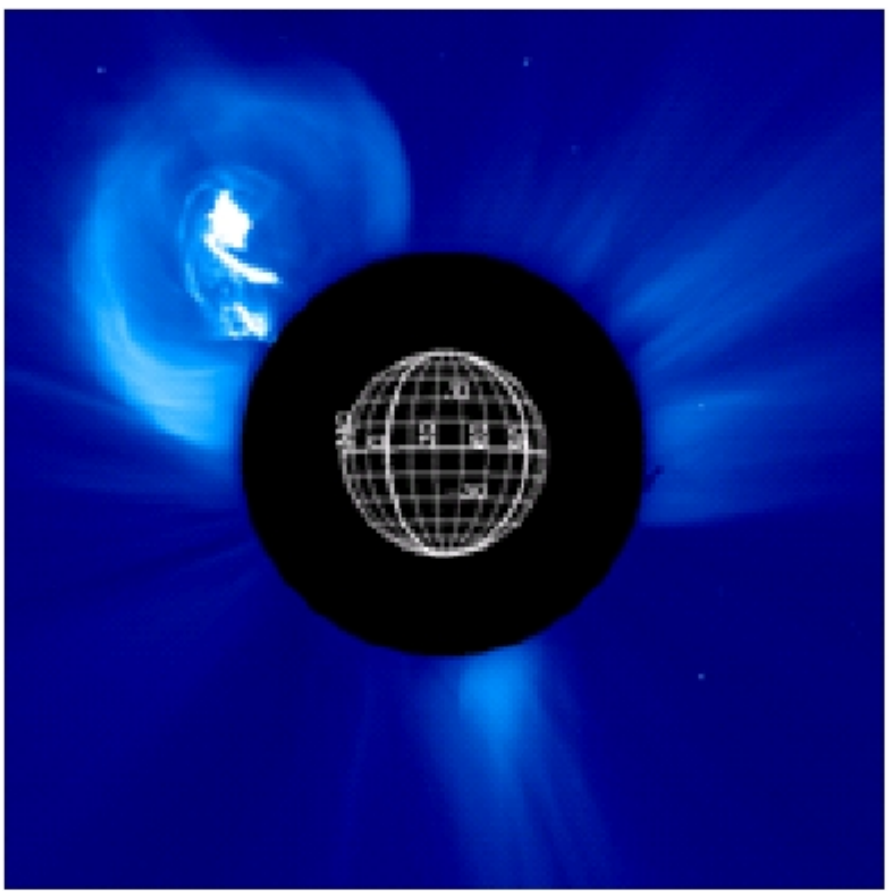

Fig. 7: A coronal mass ejection (CME) observed on 04.01.2002 with the coronagraph $\mathrm{SOHO} / \mathrm{LASCO} / \mathrm{C} 2$. This image shows clearly the three-part structure of a CME, consisting of (i) the bright leading edge, (ii) the dark void in between and (iii) the bright core showing the plasma material of the filament eruption. From Cremades and Bothmer (2005).

The characteristics of CMEs and their observational features are presented in the following section. Afterwards it is explained how CMEs originate and which models are used to describe CMEs together with the corresponding theoretical background.

\subsubsection{CME Characteristics and Observational Features}

During the last decades more than 10000 CMEs have been detected with coronagraphs of several spacecraft missions, in particular with the observations of the SOHO satellite (Gopalswamy et al., 2009). These missions allowed to resolve in more spatial and temporal detail the CME's morphology and evolution compared to earlier observations. The photospheric radiation from the Sun is Thomson-scattered by free electrons of the corona. Regions which show an increased brightness in the white-light images correspond to regions with an increased electron density along the line of sight to the observer. Coronal structures with enhanced brightness in white-light can 
depict CMEs as defined by Hundhausen et al. (1984) or helmet streamer or jet-like structures, amongst other. The CME characteristics revealed from observations like the CME occurrence rate, the CME's shape, orientation, angular expansion, plane-of-sky speed and CME mass vary to a large extent. Table 2 provides an overview of typical properties of coronal mass ejections.

\begin{tabular}{ll}
\hline CME characteristics & Range \\
\hline Speed & $300-3000 \mathrm{~km} / \mathrm{s}$ \\
Mass & $5 \times 10^{12}-5 \times 10^{13} \mathrm{~kg}$ \\
Kinetic energy & $10^{23}-10^{24} \mathrm{~J}$ \\
Angular width & $\sim 24^{\circ}-72^{\circ}$ \\
Occurrence frequency & $0.5-6$ per day (sol. min. - sol. max.) \\
\hline
\end{tabular}

Tab. 2: Typical characteristics of CMEs. From Bothmer (2006).

\subsubsection{Models for Coronal Mass Ejections}

Coronal mass ejections show many different shapes in white-light coronagraph observations and it is assumed that most of these different shapes are due to projection effects (Schwenn, 2006). In the past the CME's structure was described in various ways as erupting bubbles, bulbs, (coronal) loops and amorphous clouds (Munro et al., 1979) or with a three-part structure (Illing and Hundhausen, 1985) and as flux tubes or flux ropes (Kuperus and Raadu, 1974). Often CMEs were observed showing a closed frontal loop as illustrated in Figure 7. The morphology of such CMEs can be described with the three-part structure as proposed by Illing and Hundhausen, 1985. This scheme characterises a CME with a bright leading edge followed by a dark cavity with a bright core trailing behind. The bright core is associated with prominence material (House et al., 1981; Cremades and Bothmer, 2004). Although this three-part structure is commonly accepted as a standard morphology for CMEs there is a high percentage (70\%) of CMEs which exhibit not all characteristics of this structure (Webb and Hundhausen, 1987). Since the observation of CMEs with the $\mathrm{SOHO} / \mathrm{LASCO}$ coronagraphs the structure of these CMEs was revealed in more detail. On the one hand a helical or flux rope like structure was found and on the other hand filaments or erupting prominences were observed within the dark cavity of originating CMEs and therefore filaments were strongly associated to CMEs (Gopalswamy et al., 2003). These filaments are considered as source region indicator for (three-part structured) CMEs and can be well described with the flux rope model as introduced by Kuperus and Raadu (1974). Since the filament eruptions are strongly associated with erupting CMEs the flux rope model was applied to the progenitor of CMEs. Low and Hundhausen (1995) supposed for CMEs originated at helmet streamers a flux rope structure which is located underneath a helmet streamer with a filament at the bottom. Using this approach the CME's frontal loop develops from the high-density streamer material which surrounds the dark cavity in the center region. The filament or prominence located at the bottom corresponds to the CME's bright core below of the flux rope. Thereby this flux rope model reflects the three-part structure of CME progenitors and the three-part structure is also visible in white-light observations for evolved CMEs. Figure 8 presents on the left a schematic view of an erupting CME with its threepart structure and a prominence (red) embedded in the dark cavity. According to the schematic view is shown an erupting prominence on the right observed with the COR1 coronagraph (coloured in green) and the EUVI imager (coloured in red) on-board STEREO.

\subsubsection{The Origination of CMEs}

Large and energetic CMEs require a strong energy source to erupt from the solar atmosphere and to propagate outwards to interplanetary space. A typical CME with a volume of $10^{24} \mathrm{~m}^{3}$ exhibits an energy density in the range of $10^{-2}-10 \mathrm{~J} \mathrm{~m}^{-3}$. Based on possible energy sources like the kinetic, thermal, potential and the magnetic energy $\left(\mathrm{B}^{2} / 2 \mu_{0}\right)$ the latter one is considered as the major supplier with a rough energy density of $40 \mathrm{~J} \mathrm{~m}^{-3}$ and a magnetic flux density of $10^{-2} \mathrm{~T}$ (Forbes, 2000). Regions on the solar surface with closed magnetic fields forming a magnetic bipolar region (see also Section 2.4.3) are source regions of CMEs, (Cremades and Bothmer, 2004). Figure 9 shows a photospheric vector magnetogram of a bipolar magnetic region. The white lines indicate 
a)

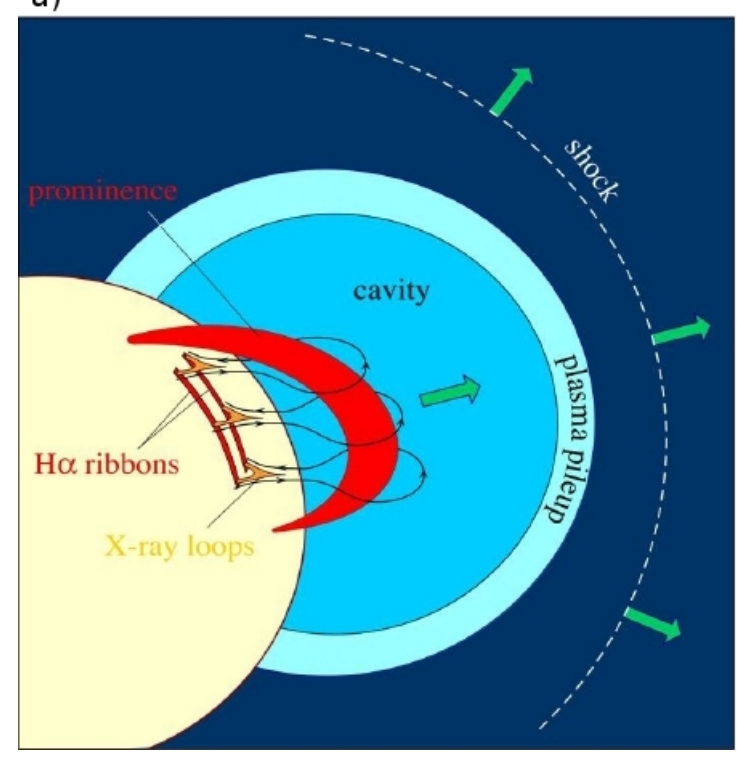

b)

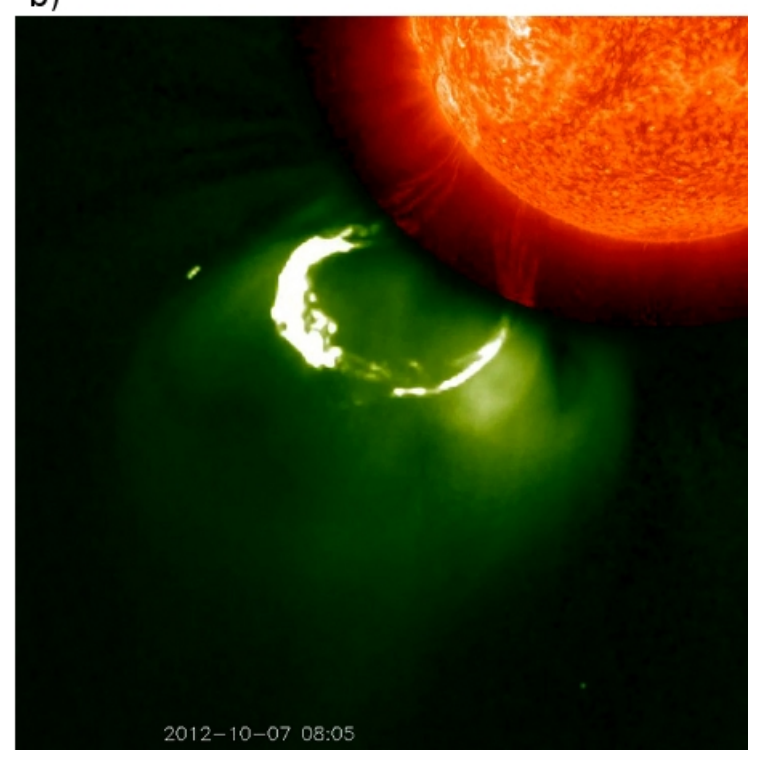

Fig. 8: A schematic illustration of an erupting CME is shown in the left frame (Forbes, 2000). A prominence (red) is embedded in the dark cavity of the three-part structured CME. A curved front represents the bright leading edge propagating outwards and driving a shock wave. For comparison a prominence eruption observed with the COR1 coronagraph (coloured in green) and the EUVI imager (coloured in red) on-board STEREO is shown on the right hand side. From GSFC/NASA (http://cor1.gsfc.nasa.gov).

reconstructed nonlinear force-free magnetic field showing a magnetic arcade. This arcade straddles over the neutral line which separates both regions (black and white) of opposite magnetic polarity (Yan et al., 2001; Tripathi et al, 2004).

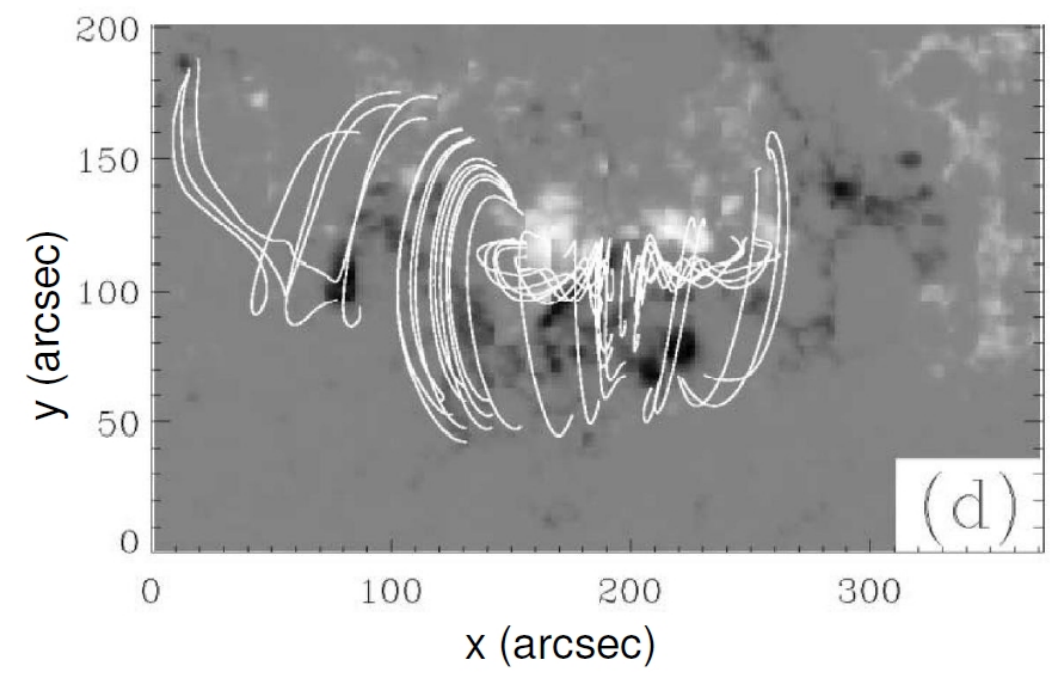

Fig. 9: A bipolar magnetic region shown in a photospheric vector magnetogram. The white lines indicate reconstructed nonlinear forcefree magnetic fields showing a magnetic arcade. This arcade straddles over the neutral line which separates both regions (black and white) of magnetic polarity. From Yan et al. (2001).

Based on such a bipolar magnetic source region Moore and LaBonte proposed in 1980 the 'Tethercutting or flux cancellation mechanism' which describes how a CME evolves and a mechanism to trigger the CME eruption. Presuming a bipolar magnetic region with a magnetic arcade, a flux rope holding a filament is kept in equilibrium by overlying envelope magnetic field lines (left frame in Figure 10). The dashed line in the left frame indicates the separation line of the bipolar magnetic region. The magnetic field near the filament is strongly sheared, in contrast the overlying envelope magnetic field is less-sheared. Before the eruption the equilibrium is sustained by a downward 


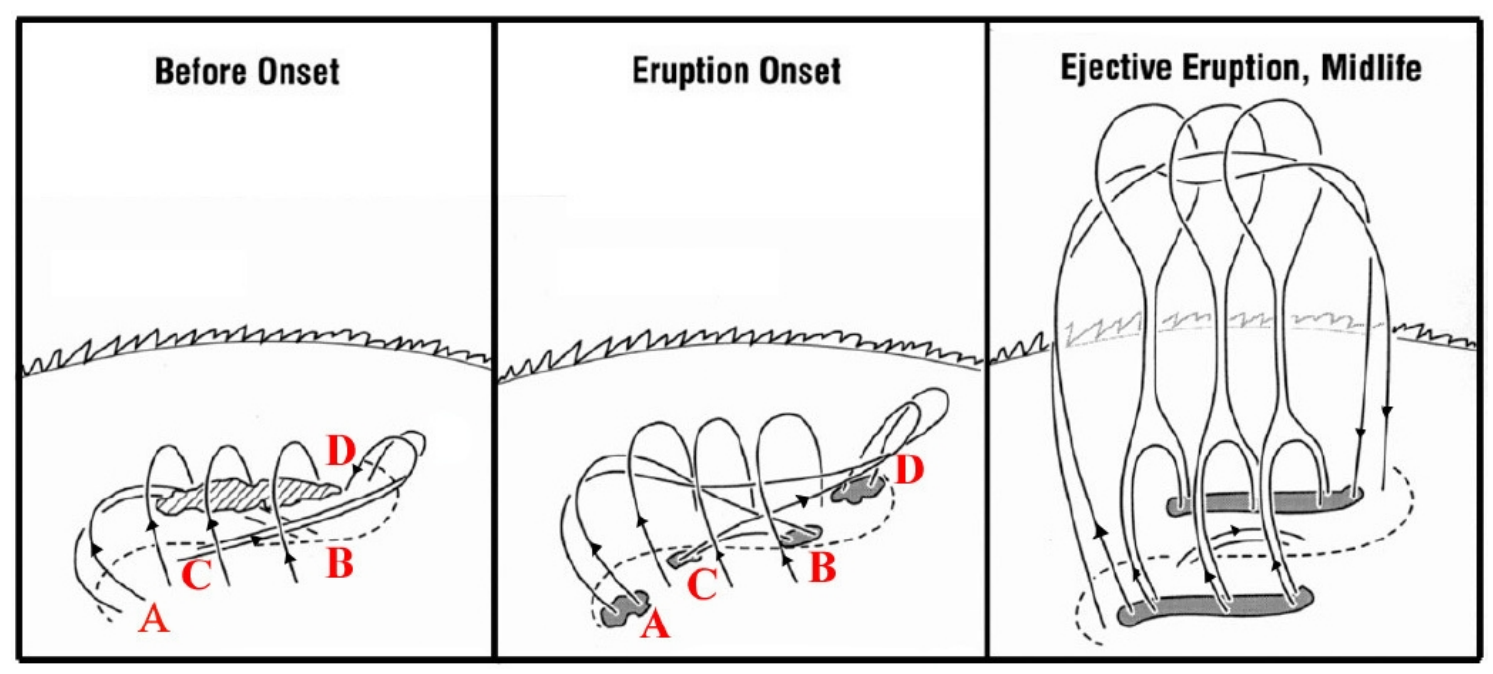

Fig. 10: The 'Tether-cutting triggering mechanism' for CMEs. The left frame presents a bipolar magnetic region with magnetic field lines holding a filament. The field lines $\mathrm{AB}$ and $\mathrm{CD}$ rearrange due to magnetic reconnection to field lines $\mathrm{AD}$ and $\mathrm{CB}$ (center frame). This process triggers the flux rope system to erupt (right frame). From Moore et al. (2001) and adapted from Chen (2011).

oriented magnetic tension force and an upward directed magnetic pressure force. With an increase of the magnetic shear the adjacent ends of the magnetic field lines AB and CD are close to each other and are antiparallel oriented resulting in a strong current sheet in between. Due to some instability magnetic reconnection starts forming short field lines CB and long field lines AD (center frame). The ongoing magnetic reconnection drives the flux rope to move upwards and through this process the envelope magnetic field is stretched up (right frame of Figure 10). This process is considered as the triggering phase of the CME and its eruption (Moore and LaBonte, 1980; Moore et al., 2001 and Chen 2011).

\subsubsection{The 'Cremades and Bothmer CME Scheme'}

Once the CME is ejected into space it can be observed and tracked near the Sun with white-light coronagraphs. It was found that white-light observations of CMEs could be successfully reproduced with a model of idealised flux ropes as published by Chen et al. (1997), Vourlidas et al. (2000) and Krall and St. Cyr (2006).

In 2004 Cremades and Bothmer identified a large set of 'structured' CMEs observed with the SOHO/LASCO coronagraphs. They studied the CME's white-light fine structure and analysed the characteristics of the CME's associated source regions. The CME's morphology, its position angle and angular width was determined from coronagraph observations and correlated with the position and orientation of the source region's neutral line from SOHO/EIT and MDI data. From these observations and analyses they found that the projected CME white-light topology depends on the position and orientation of the source region's neutral line on the solar disk. Based on these results Cremades and Bothmer deduced a generic scheme for the geometrical properties of coronal mass ejections. With the newly introduced Cremades-Bothmer scheme the analysed CMEs are described with a cylindrical topology. For this cylindrical geometry the longitudinal symmetry axis depends on the orientation, position and length of the source regions's neutral line observed on the solar disk. Figure 11 presents in frame b) two archetype CME events observed in the northern and two in the southern hemisphere. A cylindrical shaped CME with its symmetry axis parallel to the line of sight is shown in the upper left image of frame b). For comparison the CME scheme is displayed in frame a) showing the leading edge and the core material located in the middle of the cylindrical structure along its axis. The neutral line (white line in frame a)) of the underlying source region is in the same way orientated as the axis of the cylindrical structure namely nearly perpendicular 

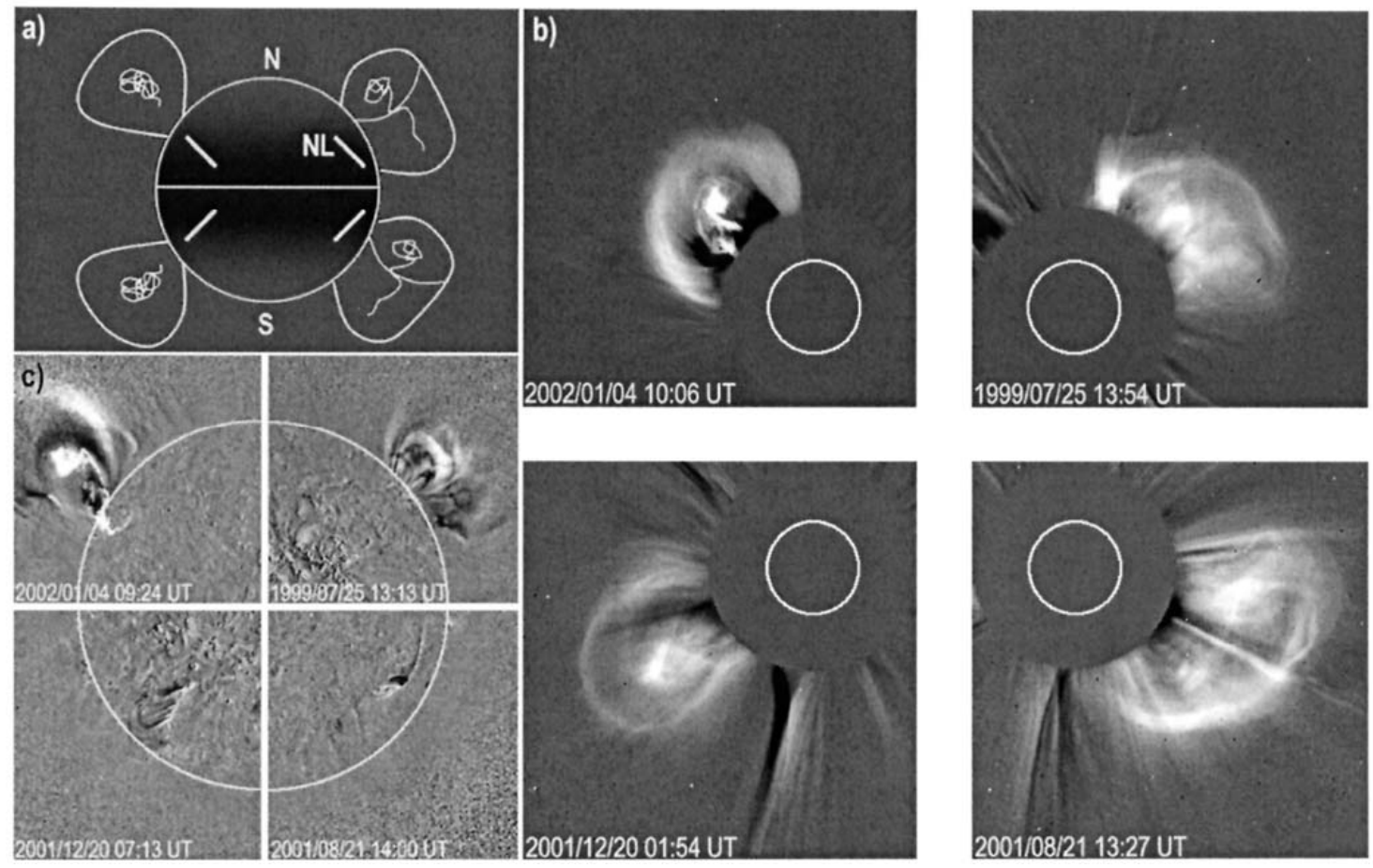

2002/01/04 10:06 UT
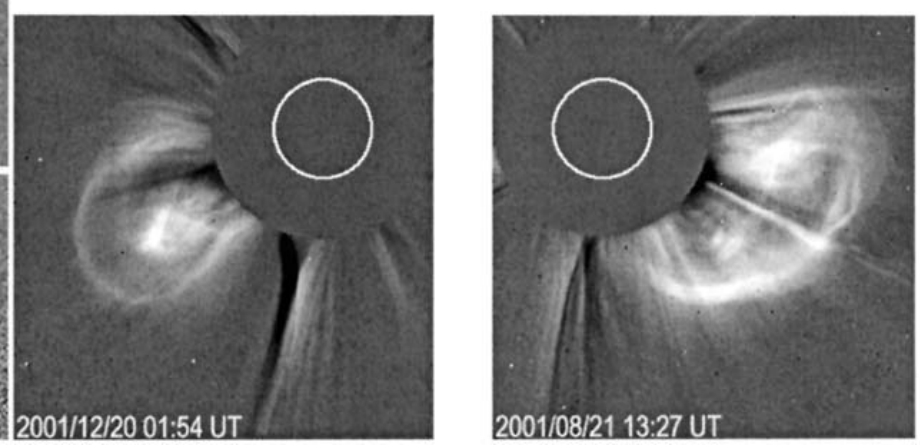

Fig. 11: The CME scheme introduced by Cremades and Bothmer (2004) describes the CME's topology with a cylindrical structure. The longitudinal symmetry axis depends on the position and orientation of the source region's neutral line (denoted in frame a) with 'NL'). In frames b) are shown archetype CMEs observed with $\mathrm{SOHO} / \mathrm{LASCO} / \mathrm{C} 2$ and in frame a) the corresponding simplified CME scheme. Frame c) presents the CME's source regions (northern hemisphere: prominence eruptions, southern hemisphere: post eruptive arcades, observed with running difference images of SOHO/EIT. From Cremades and Bothmer (2004).

to the line of the east limb. This view is interpreted as the side view of the magnetic flux rope. The second archetype CME is shown in the upper right image of frame b) with its cylindrical axis perpendicular to the line of sight. For this case the core material is distributed all over the cylindrical structure and along its longitudinal axis. Here again, the cylindrical axis is oriented parallel to the source region's neutral line (frame a)) and in this case is lying nearly parallel to the line of the west limb. The same applies for events observed in the southern hemisphere. It should be noted that this scheme must be reversed for limb events generated on the far side of the Sun because for this case the neutral line is perpendicular orientated with respect to the observer's vantage point. Following this CME scheme it implies that the orientation of the neutral line plays an important role for identifying the CME's topology projected in coronagraph images (Cremades and Bothmer, 2005).

Since the generally accepted flux rope model allows a more detailed and sufficient description of the CME's white-light appearance and inspired by the Cremades-Bothmer CME scheme, Thernisien et al. (2006) developed a geometrical model and a modeling technique which uses multipoint coronagraph observations of a CME to determine its 3-D structure, orientation and position in space. This so called Graduated Cylindrical Shell (GCS) model describes the CME's morphology similar to a "hollow croissant" with the assumption of an electron density distribution which peaks on the surface of the CME shape. This technique was developed in view of the upcoming STEREO mission in 2006 which provides for the first time stereoscopic views on CMEs, allowing the reconstruction of their 3-D geometry, orientation and position near Sun or in the STEREO/SECCHI/COR2 field of view (FOV), respectively. The STEREO mission is introduced in Chapter 3 and the Graduated Cylindrical Shell model is explained in detail in Chapter 5.2. 


\subsection{Solar Activity}

With more and more sophisticated observation techniques of the Sun various solar activity phenomena were discovered in the past. Hundreds of years ago sunspots were observed and documented e.g. by the astronomer Johann Fabricius, published in 1611 and reviewed and newly documented by Mitchell in 1916. Large twisted plasma loops best visible at the solar limb, the so-called prominence eruptions or filaments, active regions, sunspots, flares and coronal mass ejections are some of these phenomena which represent impressively the Sun's activity. With a periodicity of about 11 years the activity increases and decreases which can be traced with the monthly sunspot number as an indicator for solar activity amongst other quantities. Within the time period of one solar activity cycle the Sun's global magnetic field reverses polarity, i.e. after 22 years we find again the same polarity.

\subsubsection{Sunspots}

Sunspots were observed for hundreds of years but it is only since the invention of sophisticated ground-based telescopes that their structure could be discovered in detail. Sunspots have a typical size in diameter of up to $2 \times 10^{4} \mathrm{~km}$ with a dark umbra in their center at a temperature of $4100 \mathrm{~K}$ and a field strength up to $0.3 \mathrm{~T}$. The ambient penumbra consists of light and dark radial filaments. The magnetic field lines are almost perpendicular in the umbra and are more horizontal orientated in the surrounding penumbra. Sunspots can exist for more than 100 days and can exhibit unipolar, bipolar or more complex magnetic fields. Their distribution in the northern and southern hemisphere is described by the Joy's Law and visualised in the butterfly diagram (Hale et al., 1919; McClintock and Norton, 2013). Joy's Law states that the leading sunspots appear at lower heliographic latitudes than the following ones. In the beginning of a solar cycle sunspots occur at higher latitudes and in the end of a cycle closer to the solar equator. Figure 12 shows on the left sunspots on the solar surface observed on November, 9th 2011. A closer view of a sunspot group seen with a ground-based solar telescope on July, 15th 2002 is presented on the right in Figure 12 (Kivelson et al., 1995). The sunspot number is an important quantity to describe the

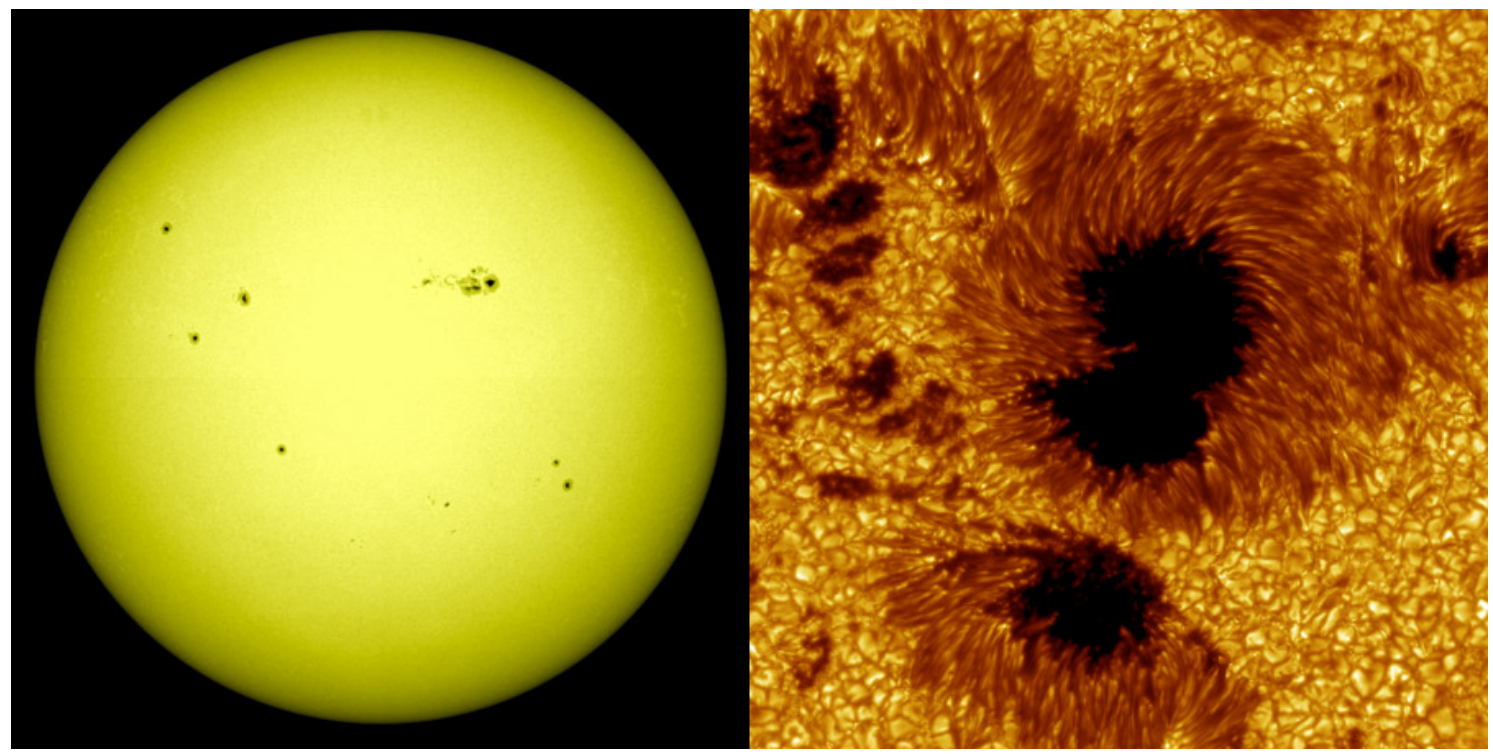

Fig. 12: Left: Sunspots on the solar surface observed with SDO/AIA at $4500 \AA$ on November, 9th 2011; From NASA/SDO. Right: A sunspot group observed with a ground-based solar telescope on July, 15th 2002. From Royal Swedish Academy of Sciences.

Sun's activity and defines the duration of solar cycles. The periodic changes in the solar activity like magnetic field orientation, solar radiation, occurrence of CMEs, sunspots and flares constitutes the solar cycle. The period between two minima of the monthly sunspot number defines a solar 
cycle which has a duration of about 11 years. The current cycle 24 started in 2009 as illustrated in Figure 13. The observed and predicted monthly sunspot rates makes this one the smallest sunspot cycle since cycle $14(1902-1913)^{1}$. An overview about the last six solar cycles is shown in Figure 13.

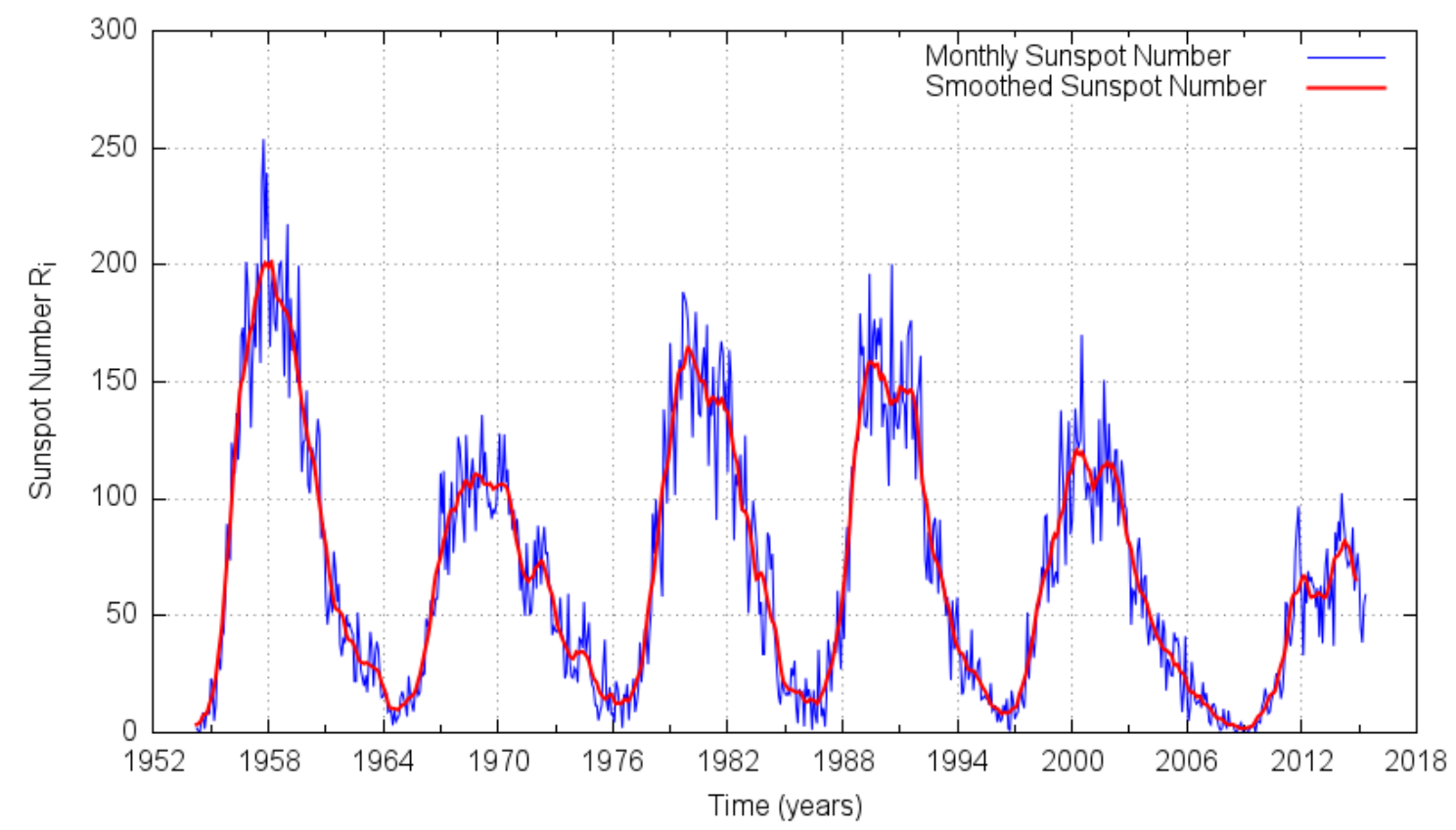

Fig. 13: International sunspot number $\mathrm{R}_{i}$ : monthly mean (blue) and 13-month smoothed number (red) for the current (24) and the last five solar cycles. From SIDC/ROB (http://sidc.be/silso/ monthlyssnplot).

\subsubsection{Prominence Eruptions}

Prominences can be observed in $\mathrm{H} \alpha$ images as thin dark filaments or in extreme ultraviolet (EUV) images at $304 \AA$ in emission. Their large-scale loop structure can be best observed near the solar limb (see Figure 14). Prominences with a typically magnetic field of strength $0.8-1 \mathrm{mT}$ exhibit plasma densities of $10^{15}-10^{17} \mathrm{~m}^{-3}$ and temperatures of $7500-9000 \mathrm{~K}$ (Parenti, 2014). Prominences can be described with a twisted flux tube model (see Figure 15) as proposed by Demoulin and Priest in 1989. The geometry is illustrated as a large-scale flux tube which becomes twisted by flux cancellation or Coriolis forces (Martin, 1986; Van Ballegooijen and Martens, 1989). With ongoing twist or flux cancellation, the prominence enlarges in length, until the twist or length becomes too large resulting in an unstable prominence which finally erupts (Kivelson et al., 1995).

\subsubsection{Active Regions}

The appearance of the Sun and its activity features depend on the wavelength of observation. With $\mathrm{H} \alpha$ filters the chromosphere can be studied and sunspots, other dark regions (like filaments or prominences) and bright regions are visible. Bright regions are called active regions and can change their brightness sometimes within minutes producing an solar X-Ray flare. The Space Weather Prediction Center at NOAA $^{2}$ describes an active region as

"In solar-terrestrial terms, a localised, transient volume of the solar atmosphere in which plages, sunspots, faculae, flares, etc. may be observed."

\footnotetext{
${ }^{1}$ Solar Cycle Prediction from Solar Physics at Marshall Space Flight Center (MSFC/NASA), http:// solarscience.msfc.nasa.gov/predict.shtml

${ }^{2}$ NOAA: National Oceanic and Atmospheric Administration, http://www.noaa.gov
} 


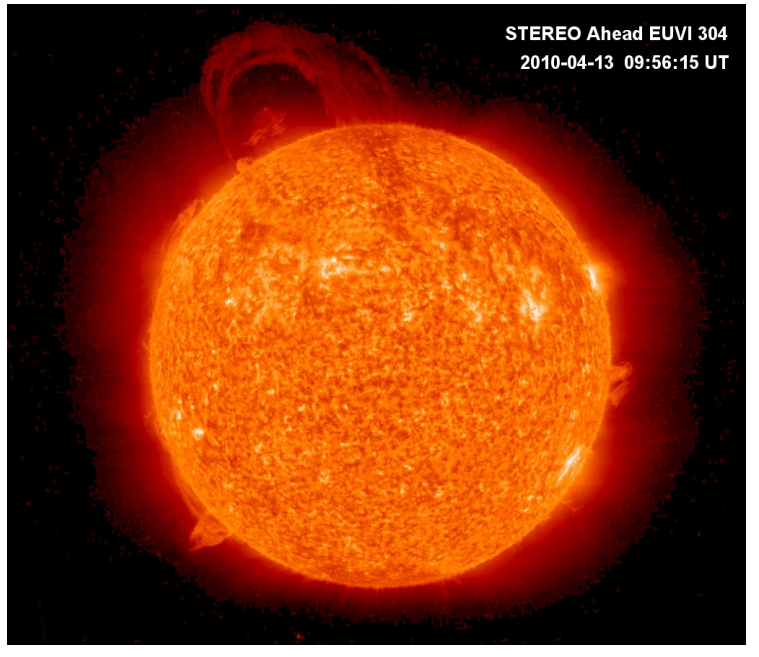

Fig. 14: Prominence eruption as observed with STEREO/SECCHI/EUVI-A at $304 \AA$ on April, 13th 2010. From NASA, STEREO/SECCHI/EUVI.

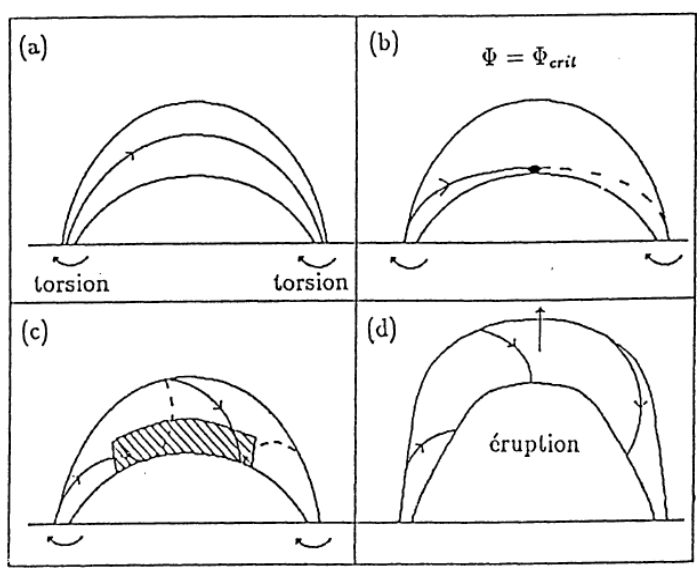

Fig. 15: A prominence eruption described with a twisted magnetic flux tube, published by Demoulin and Priest (1989).

from the glossary of the NOAA National Weather Service ${ }^{3}$. Furthermore NOAA detects and numbers the active regions as 'NOAA Active Region', e.g. NOAA 12052, and determines the location $(\mathrm{N} / \mathrm{S}, \mathrm{E} / \mathrm{W})$ on the visible disk in the Stonyhurst coordinate system (Figure 16). Active regions can be observed in regions with strong magnetic field. These areas are characterised by numerous magnetic activity processes like magnetic flux emergence, magnetic reconfigurations and flux cancellation. Due to these processes CMEs occur frequently at active regions (Aschwanden, 2006).

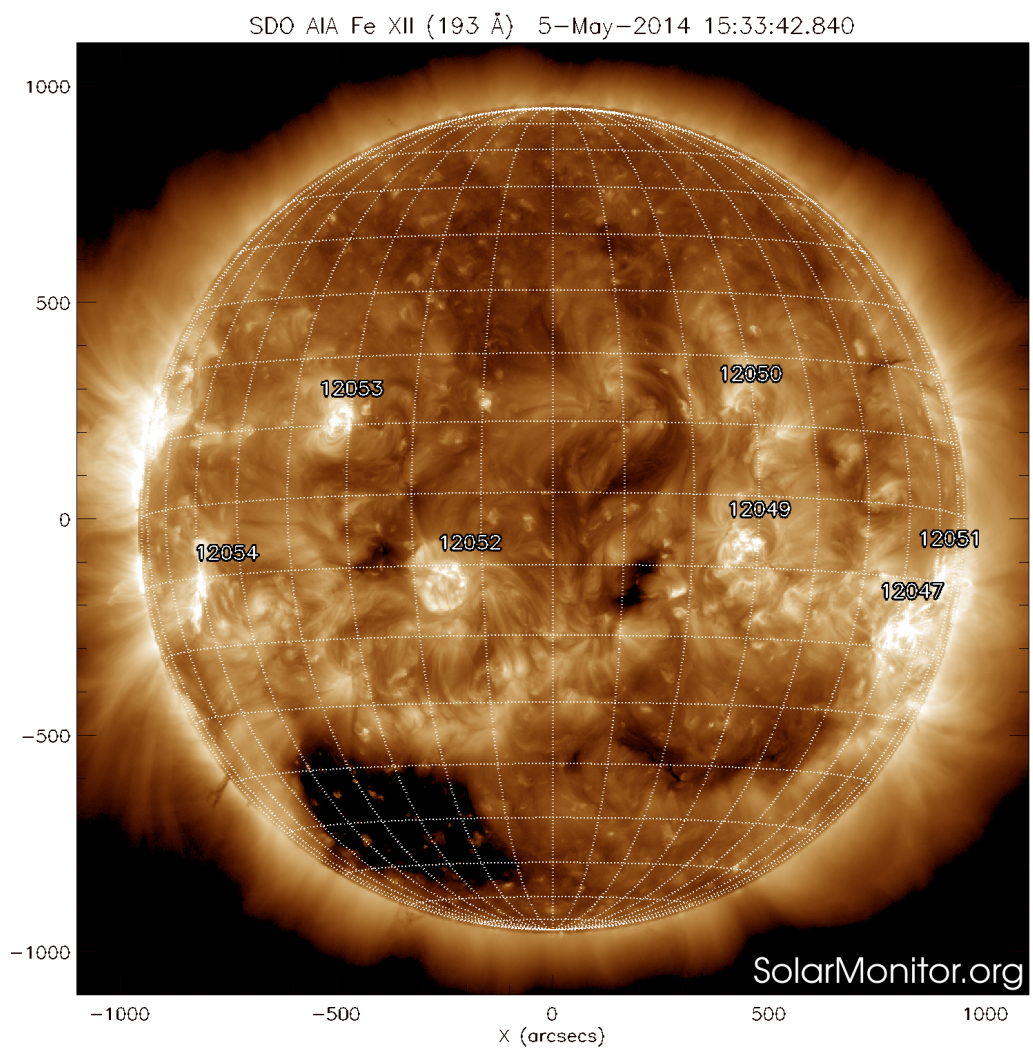

Fig. 16: NOAA Active Regions observed on May, 5th 2014 with SDO/AIA at $193 \AA$. From NASA/SDO, NOAA/SWPC, www. solarmonitor.org.

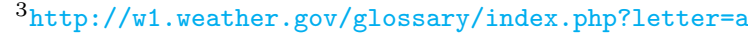




\subsubsection{Post Eruptive Arcades}

Post eruptive arcades (PEAs) are visible as bright arcade like loops in the extreme ultraviolet bandwidth e.g. at $195 \AA$ (Tripathi et al., 2004). PEAs, also known as post-flare loops, occur as active flickering bright arcades after the lift-off of an prominence eruption. Their appearance is observed in close time and spatial conjunction with CMEs, therefore they are considered as indicator for a CME's source region as discussed by Rust and Webb (1977), Svestka et al. (1998) and Hudson et al. (1998). Tripathi, Bothmer and Cremades studied 2004 the correlations between EUV PEAs and CMEs and found an almost one to one correspondence between PEAs and CMEs occurring between 1997 and 2002. A detailed comparison revealed that PEAs evolve after the occurrence of CMEs and persist over a life-time of about 2 to 20 hours. Hence longer lasting PEAs still exist at times when the associated CME already propagated to distances of a couple of solar radii, depending on its individual propagation velocity. The heliographic length of the PEA's longitudinal axis varies in the range of 2 up to 40 degrees. It was found that their heliographic position is in agreement with the active region belts in both hemispheres. Figure 17 shows a post eruptive arcade on the Sun's west limb observed at $195 \AA$ with the EUV Imaging Telescope (EIT)

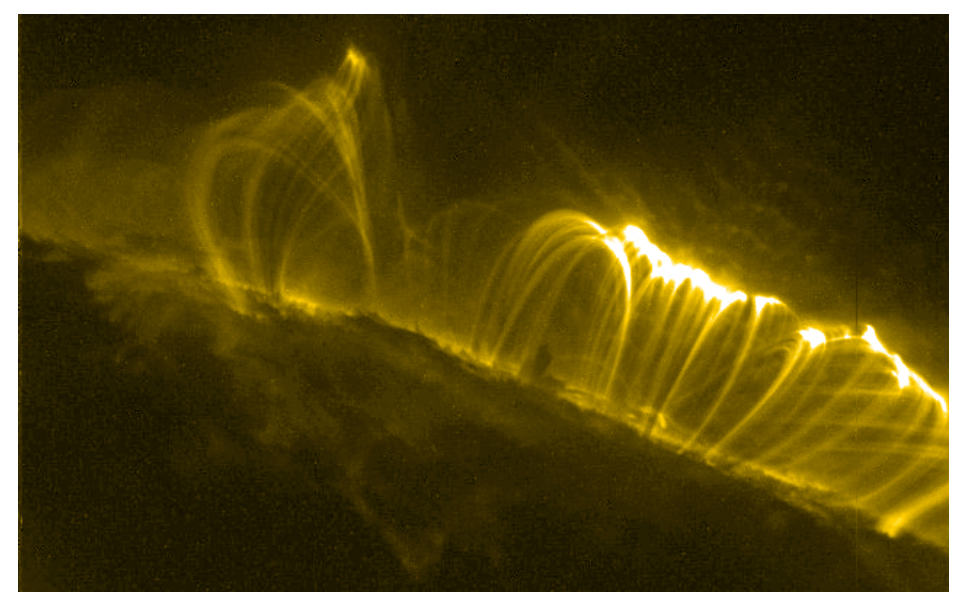

Fig. 17: A post eruptive arcade observed at $195 \AA$ on November, 4th 2003 with TRACE. From NASA/TRACE.

of TRACE. Tripathi et al. (2004) found that PEAs originate not only in single bipolar regions but also from pairs of neighbouring bipolar regions.

\subsubsection{Flares}

A solar flare is best observable on the solar disk in X-ray and EUV wavelengths e.g. at $195 \AA$ as shown in Figure 18 (red box). The flare is visible as bright horizontal line near the west limb. The measurement of the intense brightness causes the horizontal line due to the technical effect of detector bleeding.

The CSHKP model, which was developed by Carmichael (1964), Sturrock (1966), Hirayama (1974), Kopp and Pneuman (1976), describes the origination process of flares. This general accepted model is also known as the "Standard 2D Flare Model". At the beginning the flare process is driven by a rising prominence above the neutral line (NL) of an active region, Figure 19 a). This rising prominence generates a current sheet above the neutral line and due to changing magnetic field electric currents are induced parallel to the NL. With the rise of the prominence a magnetic cavity occurs just below the erupting prominence and a magnetic collapse is induced on both sides of the current sheet. A heat and mass flow is generated near the compression zone with plasma flows directed to both sides of the current sheet, Figure $19 \mathrm{~b}$ ). It is assumed that magnetic reconnection is triggered at this location accompanied by a large magnetic energy dissipation. This dissipation yields to an impulsive heating of the local coronal plasma and an acceleration of nonthermal particles. As a result of these processes soft X-ray emitting flare loops are originated. The "Standard 2D Flare Model" is summarised by Aschwanden (2006) (Chapter 10.5.1) and discussed in more detail by Hirayama (1974). 


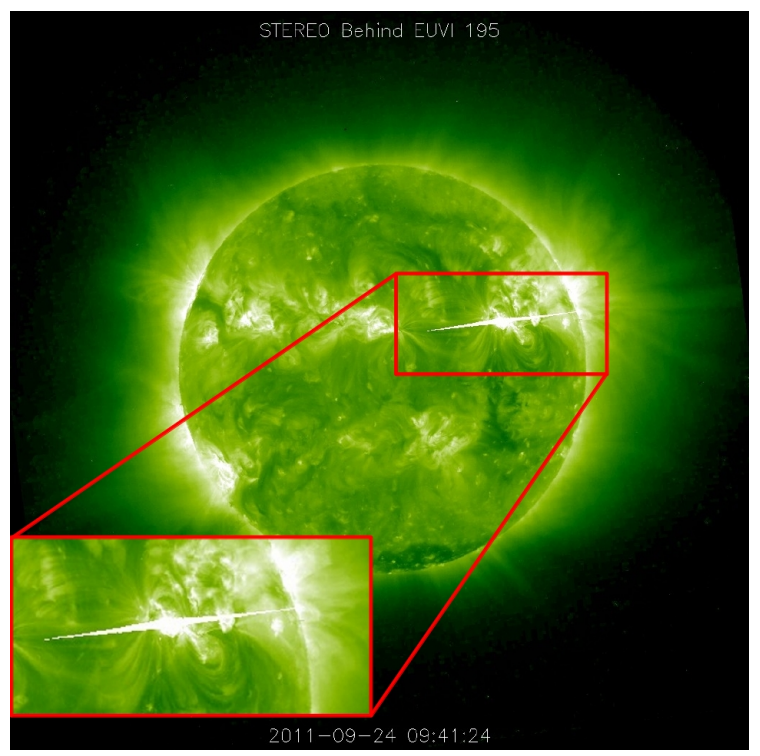

\begin{tabular}{cc}
\hline Classification & X-ray peak flux $\left[\mathrm{W} / \mathrm{m}^{2}\right]$ \\
\hline A & $<10^{-7}$ \\
B & $10^{-7}-10^{-6}$ \\
C & $10^{-6}-10^{-5}$ \\
M & $10^{-5}-10^{-4}$ \\
X & $>10^{-4}$ \\
\hline
\end{tabular}

Fig. 18: A solar flare observed on September 24th, Tab. 3: Classification of X-ray flares based on the 2011 at 09:41 UTC with STEREO/EUVI at $195 \AA$. measurements of the X-ray flux with the GOES spaceThis flare is observed in the source region of the CME craft, www.swpc.noaa.gov/weekly/Usr_guide.pdf. detected in STEREO/SECCHI/COR2 at 13:54 UTC.

From NASA/STEREO.

a) Pre-flare

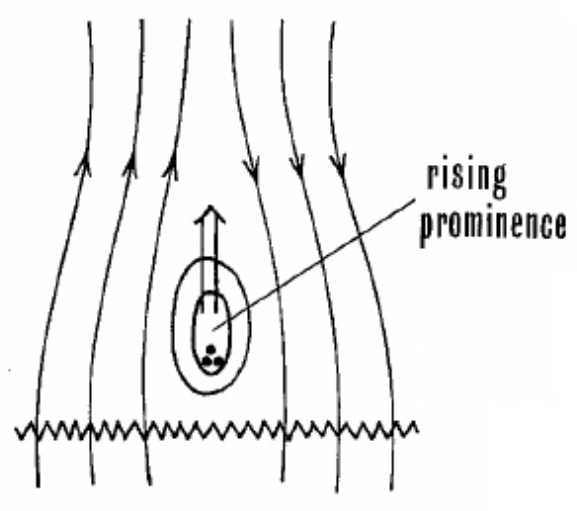

b) Maximum Phase

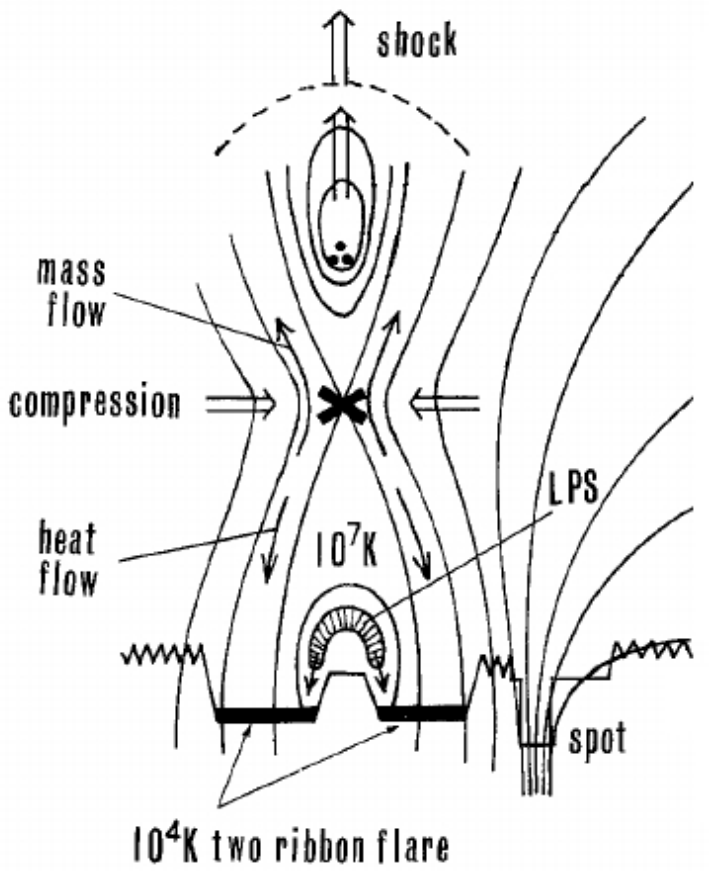

Fig. 19: The origination of a flare as described by the "Standard 2D Flare Model": The flare process is driven by a rising prominence a) which results in a magnetic cavity beneath the prominence where magnetic reconnection is triggered $b$ ). The local coronal plasma is heated and non-thermal particles are accelerated due to a large energy dissipation. As a result soft X-ray emitting flare loops are generated. From Hirayama (1974). 
Solar flares can be classified as A, B, C, M or X -Class flares depending on the peak flux $\left(\mathrm{W} / \mathrm{m}^{2}\right)$ at 1-8 $\AA$ measured with the GOES spacecraft near Earth. The flare classes are logarithmic scaled and linear within a class (apart from the X-class). A M4 X-ray flare is twice as strong as a M2 flare. The classes of X-ray flares and their peak flux are specified in Table 3. 


\section{Instrumentation and Data}

In this section the STEREO mission will be introduced which provides with their telescopes unique image data of the solar corona, CMEs and their source regions. The Solar Terrestrial Relations Observatory (STEREO) mission which forms the basis of this work and their relevant instruments are explained in detail. This concerns especially the coronagraph COR2 and the Extreme Ultraviolet Imager (EUVI) as part of the Sun Earth Connection Coronal and Heliospheric Investigation (SECCHI) instrument suite.

\subsection{Introduction}

Why is it necessary to have coronagraphs on satellites in space in addition to or instead of groundbased solar observatories? On the one hand satellite-based coronagraphs enable white-light observations without the disturbing light scattering from the atmosphere. On the other hand observatories in space allow to observe the Sun detached from the Earth's rotation and give a continuous view on the solar disk. In contrast to single point observations, the STEREO twin satellites orbiting the Sun provide for the first time ever a stereoscopic view to the Sun and heliosphere from two vantage points in space as illustrated in Figure 20. Each of the STEREO spacecraft is equipped

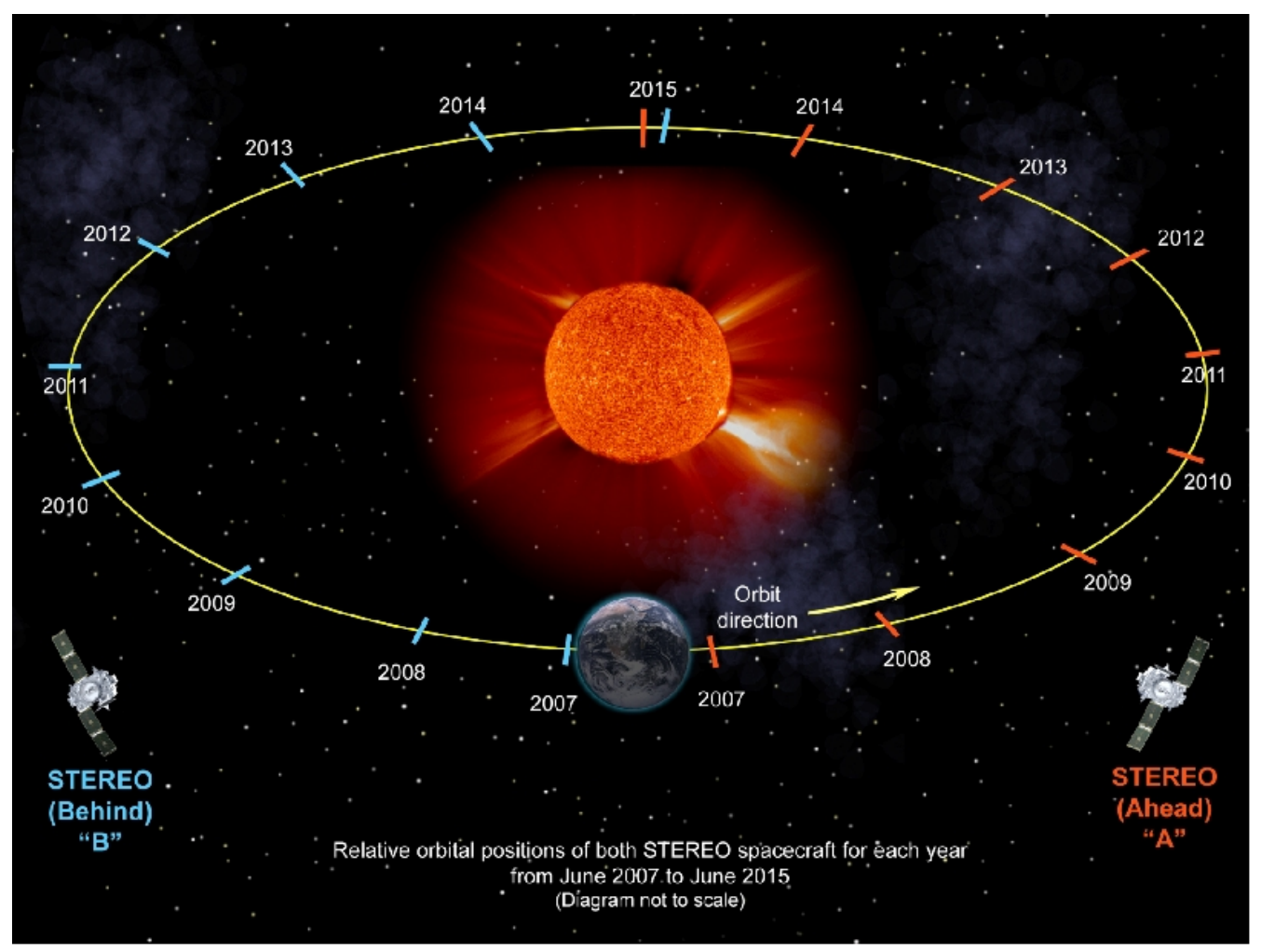

Fig. 20: Schematic illustration of the orbit for the STEREO twin spacecraft with their rough positions in respect to Earth for the times as indicated. From NASA, STEREO Consortium, http://stereo.gsfc . nasa.gov.

with four telescopes which allow to track a CME all the way from Sun to Earth, if they are at appropriate observer positions. In order to trace a CME the telescopes, which are part of the SECCHI (Sun Earth Connection Coronal and Heliospheric Investigation) instrument suite, exhibit field of views ranging from 1.4 to 318 solar radii, covering the complete Sun-Earth line. 


\subsection{The STEREO Mission}

The STEREO mission was developed to investigate the Sun and heliosphere and was launched in October 2006. One of its key aspects is to observe the Sun from two different vantage points to get a stereoscopic view of the Sun and its corona. For this purpose STEREO-Ahead (A) is flying ahead the Earth at 0.96 AU distance to the Sun and STEREO-Behind (B) is trailing behind Earth at an orbit of $1.04 \mathrm{AU}$. As a result, the separation angle between STEREO-A and -B increases with a drift rate of about $44 \pm 2^{\circ}$ per year. Both spacecraft had reached a separation angle of $180^{\circ}$ on February 6th, 2011 and consequently allowed for the first time a full $360^{\circ}$ view of the Sun ${ }^{4}$. This stage of the mission is a great advantage and unique feature of this mission because Earth directed coronal mass ejections can be observed all the way from Sun to Earth. Over the whole mission period the viewing conditions are changing and have to be considered when using and comparing STEREO data of different points in time. The scientific objectives in relation to CME observation with the SECCHI instruments which lead to this mission concept are defined as follows:

\section{The objectives of the STEREO mission concerning SECCHI:}

- What is the timing of physical properties involved in CME initiation? What are the structures involved in the CME initiation?

- What is the 3-dimensional structure and kinematic properties of CMEs?

- What is the 3-dimensional structure of active regions, coronal loops, helmet streamers, etc.?

- What are the critical forces controlling the propagation of CMEs in the corona and interplanetary medium?

(Citation from Howard et al. (2008), page 69)

To achieve these objectives both nearly identical constructed STEREO spacecraft are equipped with the following four instrument suites each dedicated to an unique field of activity:

SECCHI - Sun Earth Connection Coronal and Heliospheric Investigation This instrument suite contains two white-light Coronagraphs (COR 1, COR 2) and two Heliospheric Imager (HI 1, HI 2) for CME observation and one Extreme Ultraviolett Imager (EUVI) for observing the chromosphere and the low corona.

IMPACT - In-situ Measurements of PArticles and CME Transients

The IMPACT instruments measure energetic particles, the interplanetary magnetic field and the three dimensional distribution of solar wind electrons.

PLASTIC - PLAsma and SupraThermal Ion Composition

These instruments are dedicated to the investigation of the plasma characteristics of protons, alpha particles and heavy ions.

\section{S/WAVES - STEREO/WAVES}

SWAVES detects interplanetary radio bursts and observes their generation at the Sun and their propagation from Sun to Earth.

The STEREO mission can be divided in the following four phases which are illustrated in Figure 21.

Phase 1: In the first 1.5 years (approximately the first 480 days, Jan. 2007 - Apr. 2008) of the STEREO mission both spacecraft exhibit a separation angle less than $50^{\circ}$. During this time

\footnotetext{
${ }^{4}$ Press release published by the NASA on Feb. 6th, 2011: http://www.nasa.gov/mission_pages/stereo/news/ entire-sun.html
} 
period both spacecraft are ideal located for high cadence 3-D imaging of coronal structures, generating stereoscopic image pairs of the corona before, during and after CME lift-offs.

Phase 2: The second phase spans over the second and third year ( $\approx$ days 480 - 970, May 2008 - Aug. 2009) when the spacecraft are separated by $50^{\circ}$ up to $110^{\circ}$. In this period it is possible to observe a CME from one spacecraft in the plane of sky while the CME is directed to the other spacecraft as a halo CME. In addition both spacecraft exhibit very good vantage points for triangulation of CMEs.

Phase 3: For the next 580 days ( $\approx$ Sept. 2009 - Mar. 2011) both spacecraft were separated by $110^{\circ}$ up to $180^{\circ}$ and enable to track CMEs from Sun to Earth. With reaching the separation angle of $180^{\circ}$ (in February 2011) a complete view of the entire Sun is revealed.

Phase 4: The STEREO satellites offer observations of the far side of the Sun during this time period, which started in April 2011. This allows analyses of possible candidates for CME source regions and to derive changes for future (next days) space weather conditions (Kaiser et al., 2008).
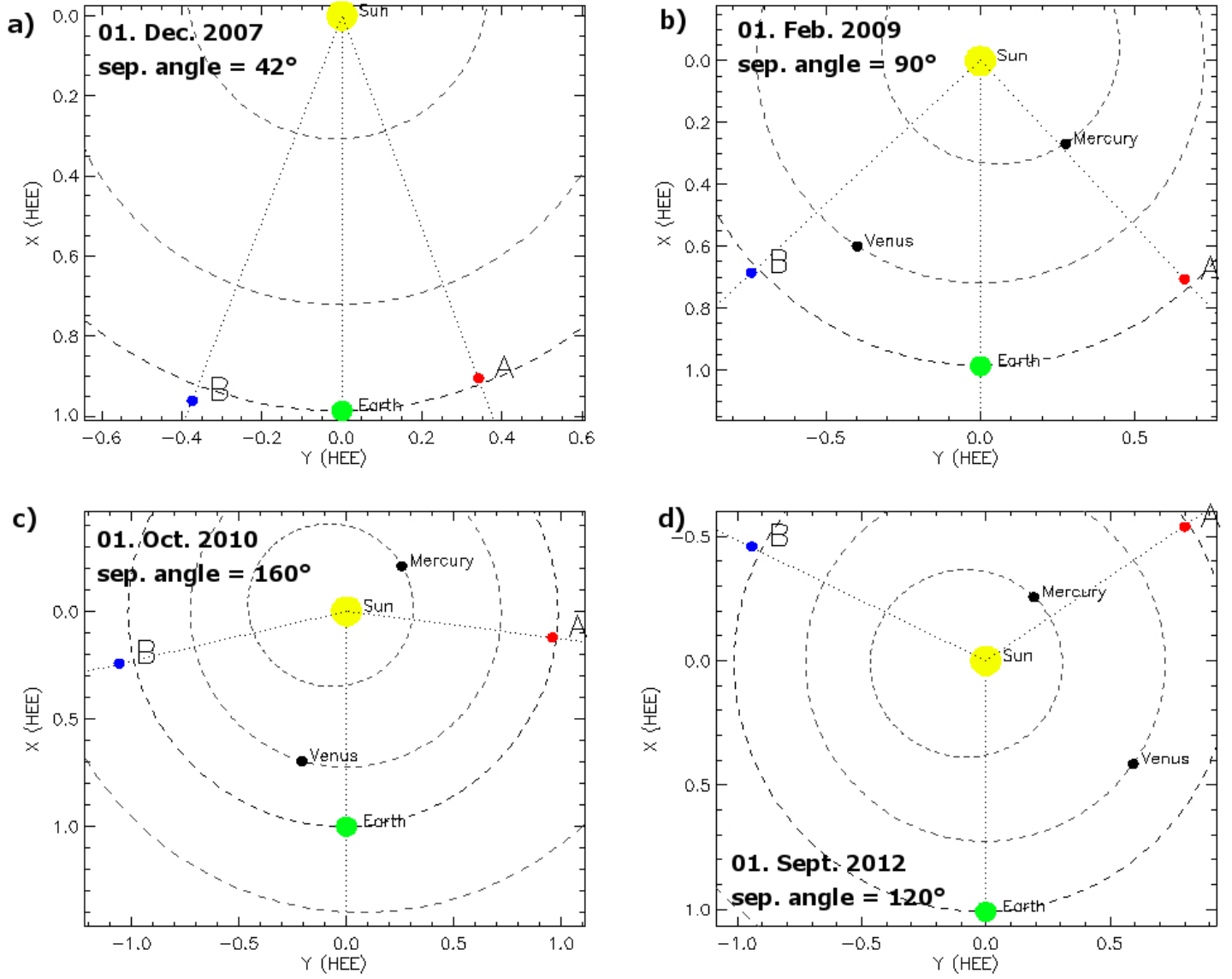

Fig. 21: The STEREO spacecraft positions for each mission phase: a) shown for December 1st, 2007 with $42^{\circ}$ separation angle; b) during phase 2, here illustrated for February 1st, 2009 with $90^{\circ}$ separation angle; c) on October 1st, 2010 with a separation angle of $160^{\circ}$ in phase 3 and d) during phase 4 the separation angle of $120^{\circ}$ is decreasing as shown for September 1st, 2012. From NASA STEREO Science Center, http://stereo-ssc.nascom.nasa.gov/where.shtml. 
In January 2007 both spacecraft initiated the prime science phase with all instruments working properly. Since that time more than $10^{7}$ images have been recorded with the SECCHI instruments, as of status from May $2016^{5}$. The current spacecraft position can be found the STEREO Science Center website hosted by NASA ${ }^{6}$.

In 2015 both spacecraft entered the orbit behind the Sun as seen from Earth and changed their position relative to Earth in March 2015 (separation angle $=0^{\circ}$ ). For a $\approx 500$ day lasting time period both satellites delivered no scientific data beginning in August 2014 until January 2016. During this time period only low-resolution beacon data were available, with the exception of the solar conjunction period. Since January 2016 the STEREO-A spacecraft is back to nominal science mode. For further detailed information see also the STEREO Coordinated Observations Calendar ${ }^{7}$ and the STEREO Solar Conjunction Schedule ${ }^{8}$. STEREO is funded on a two-year cycle. Depending on the Senior Review in April 2015 the funding is extended to the fiscal years 2016-17. The Payload Operation Center at NRL is looking forward that the STEREO mission will be continued at least until 2019.

Current status of the STEREO spacecraft in Aug. 2016:

On October 1st, 2014 the contact to STEREO-B was lost after a planned observatory reset. In the following weeks contingency operations were undertaken but unfortunately no signal was received by the DSN (Deep Space Network) radio science receivers. After 22 months the contact with STEREO-B could be reestablished on August 21, 2016. Further recovery procedures are planned to get full control on the spacecraft and to evaluate all technical systems and instruments. If the communications can be reestablished the operational plan for exiting the solar conjunction testing will continue to return the STEREO-B observatory back to nominal science data collection mode as soon as safely possible. The STEREO-A observatory is continuing with a limited science data recording. The detailed report can be found on the websites of the STEREO Science Center ${ }^{9}$. The aforementioned financing plan is not affected by the status of the STEREO Behind spacecraft.

In the following the SECCHI instrument suite with respect to CME and source region observation will be introduced. For information about the other STEREO instrument suites the reader is referred to the publications for IMPACT, PLASTIC and S/WAVES by Luhmann et al. (2007), Galvin et al. (2008) and Bougeret et al. (2008), respectively.

\subsection{SECCHI Instruments}

The SECCHI instrument suite was named after the Italian astronomer Pietro Secchi (1818 - 1878) and is used as an acronym for Sun Earth Connection Coronal and Heliospheric Investigation. SECCHI provides five telescopes for imaging the solar disk, the corona and the inner heliosphere. The main application of these telescopes is to observe features in the low corona and photosphere region and to detect transients in the solar corona and the near Sun space. For the first time Earth directed CMEs can be tracked up to distances of 1 AU from Sun to Earth orbit.

In order to view the solar disk in extreme ultraviolet wavelengths the Extreme Ultraviolett Imager (EUVI) was designed to observe the Sun at four different wavelengths. Two coronagraphs, COR-1 and COR-2, allow to detect CMEs in white-light in field of views up to 15 solar radii. CMEs propagating in direction to Earth can be tracked with two Heliospheric Imager, HI-1 and HI-2, up to a distance of $1 \mathrm{AU}$ and beyond which enables to observe their arrival at Earth. The first three instruments are aligned to Sun and compose the Sun Centered Imaging Package (SCIP). A

\footnotetext{
${ }^{5}$ Database of SECCHI Flight Images: http://sharpp.nrl.navy.mil/cgi-bin/swdbi/secchi_flight/imgshort/form

${ }^{6}$ STEREO Science Center: http://stereo-ssc.nascom.nasa.gov/where.shtml

${ }^{7}$ STEREO Coordinated Observations Calendar: http://stereo-ssc.nascom.nasa.gov/plans.shtml

${ }^{8}$ STEREO Solar Conjunction Schedule: http://stereo-ssc.nascom.nasa.gov/solar_conjunction_science. shtml

${ }^{9}$ STEREO-B Report: http://stereo-ssc.nascom.nasa.gov/behind_status.shtml
} 
schematic depiction of the STEREO-B spacecraft and the SCIP is given in Figure 22.

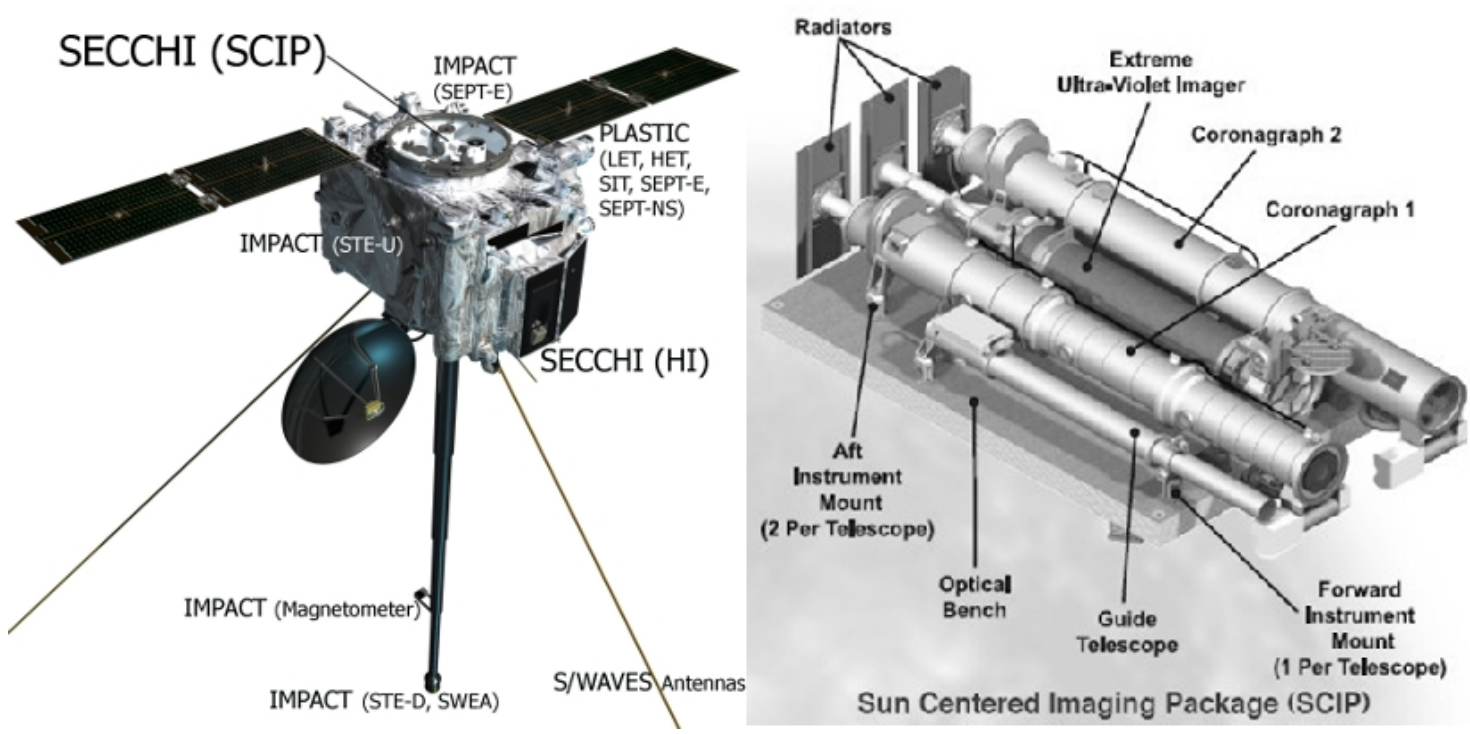

Fig. 22: Schematic illustration of STEREO-B (left) and its Sun Centered Imaging Package - SCIP (right). From Kaiser et al. (2008).

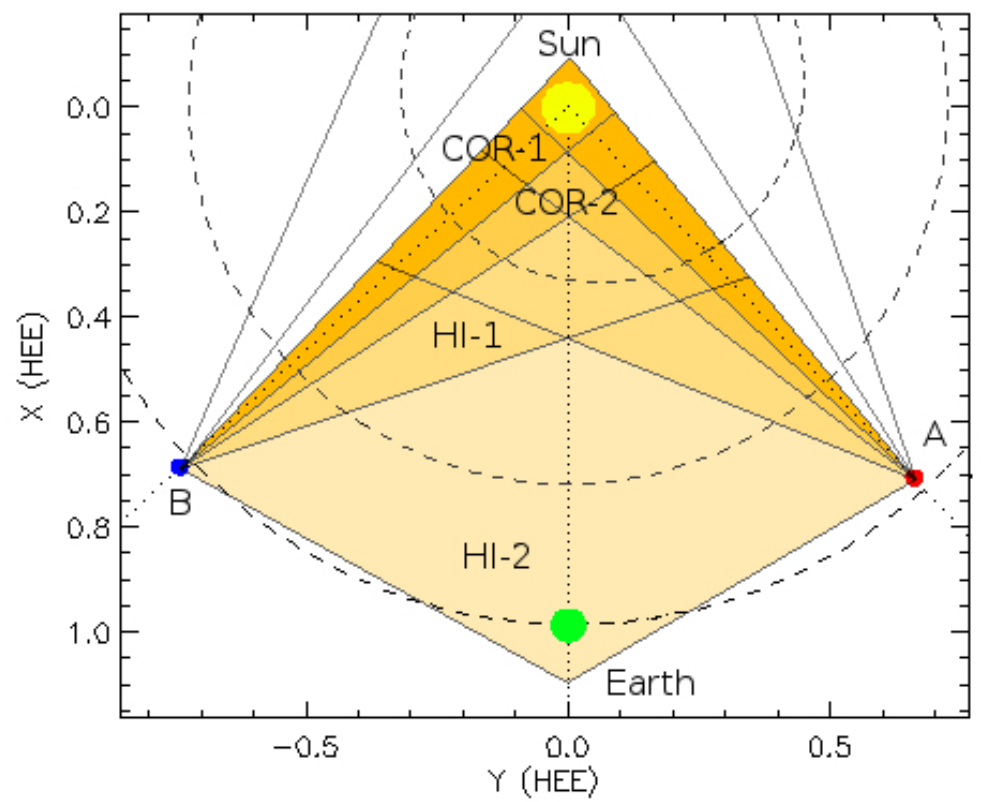

Fig. 23: Schematic illustration (not to scale) of the STEREO/SECCHI telescopes' field of view (FOV) for the satellites' positions in March 2009. The FOVs of COR-1, COR-2, HI-1 and HI-2 are shown with different shades of colour for the FOV facing to Earth. Adapted from STEREO Science Center: http://stereo-ssc.nascom. nasa.gov/where.shtml.

The SCIP is pointing to the Sun with a $8^{\circ}$ cone and a clear $180^{\circ}$ field of view (FOV). While the EUV imager observes the Sun with a circular full Sun field of view to \pm 1.7 solar radii, the COR-1 coronagraph covers a viewing angle ranging from 1.4 to 4 solar radii and the COR-2 coronagraph with a viewing angle from 2 to 15 solar radii. The Heliospheric Imager HI-1 and HI-2 are aligned with an orientation of $90^{\circ}$ to the Sun-Earth line and with a $20^{\circ}$ or $70^{\circ}$ angular field of view, respectively. Figure 23 illustrates schematically the FOV of the SECCHI telescopes. The most important technical data of the SECCHI instruments are summarised in Table 4 at the end of this chapter. 


\subsubsection{The Coronagraph - COR2}

On board the STEREO satellites are two coronagraphs and two heliospheric imager in operation. Since the inner coronagraph COR1 and both heliospheric imager HI-1 and HI-2 were not used for this study they are not explained in detail. For detailed information about these instruments the reader is referred to Howard et al. (2008).

The outer coronagraph COR2 is an externally occulted Lyot coronagraph and was developed based on the design of the successful operating SOHO/LASCO C2 and C3 coronagraphs (Brueckner et al., 1995). Figure 24 shows a schematic illustration of an externally occulted Lyot coronagraph. In order to observe weak coronal structures in white-light, COR2 measures the polarisation brightness $(\mathrm{pB})$ with a spatial resolution of 15 arc seconds per pixel on a $2 \mathrm{k} \times 2 \mathrm{k}$ CCD camera. The telescope records three lineary polarised images at $-60^{\circ}, 0^{\circ}$ and $+60^{\circ}$ in sequence. These three images are taken within 15 seconds to limit the pixel smearing of moderately fast moving CMEs $(\approx 750 \mathrm{~km} / \mathrm{s})$ in the image sequence. Each set of three images constitutes an observation which is repeated in the scheduled time of cadence. The images are processed and compressed by a factor of 10 on-board STEREO. After transmission to Earth the image processing is continued to provide total and polarised brightness images for further scientific analysis. Further details about the data processing flow are discussed in Chapter 5.

There are also other image recording and processing modes available, e.g., low-resolution images for space weather purposes. The COR2 telescopes in STEREO-A and -B are identically except for the different sizes of the occulters and different offsets of the coronagraph's boresight. An overview of the technical layout of the COR2 coronagraph is given in Figure 25 and an image pair of COR2 -A and -B showing a CME is presented in Figure 26.
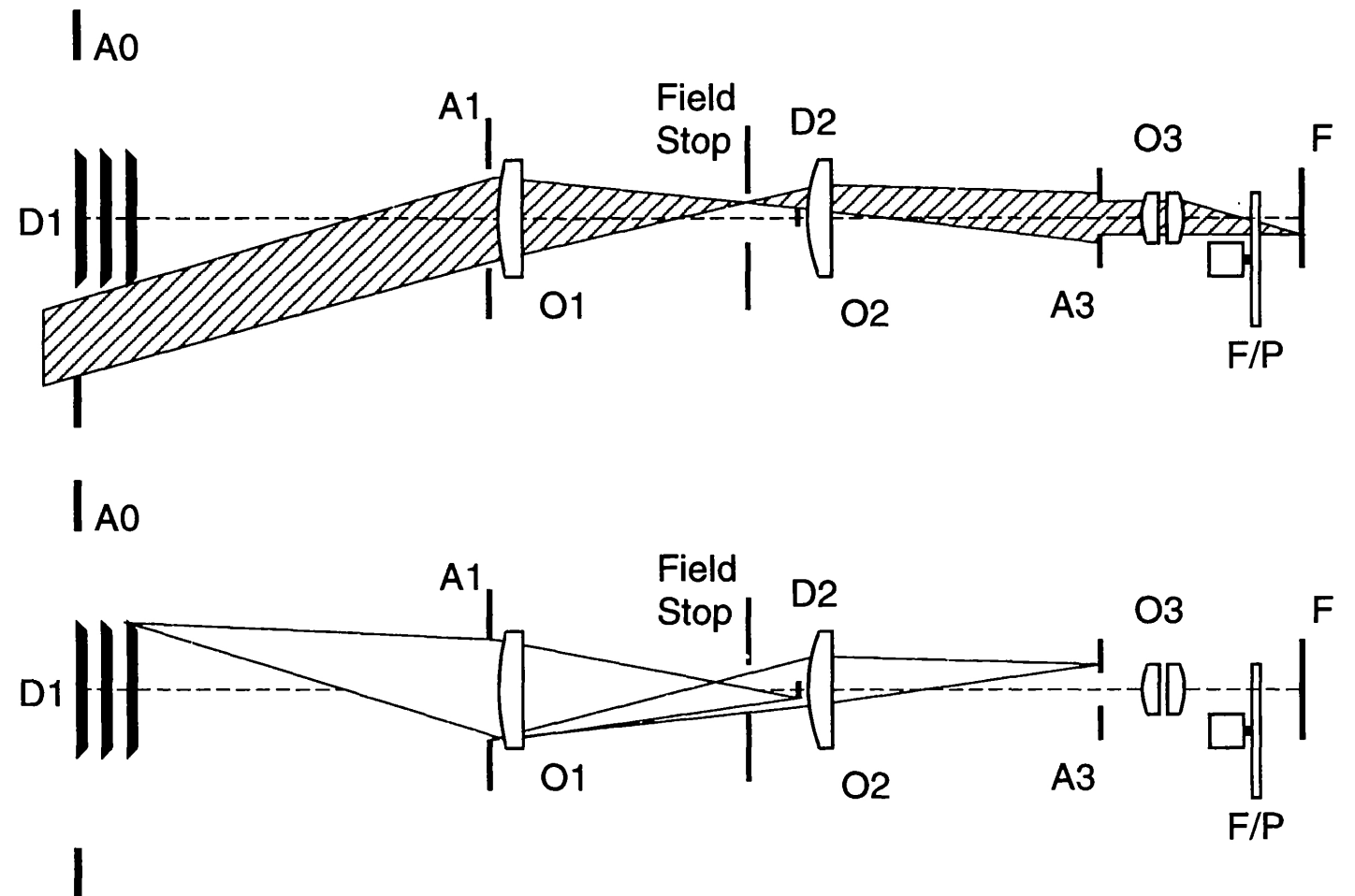

Fig. 24: Schematic illustration of an externally occulted Lyot coronagraph as used for the coronagraph $\mathrm{C} 2$ on-board SOHO. The STEREO/SECCHI/COR2 coronagraph is based on this design. On the top row is shown a ray bundle passing the coronagraph from left to right generating a coronal image in the focal plane $(\mathrm{F})$. On the bottom row is pictured the suppression of stray light beginning on the left at the external occulter (D1) and ending at the Lyot stop (A3). From Brueckner et al. (1995). 


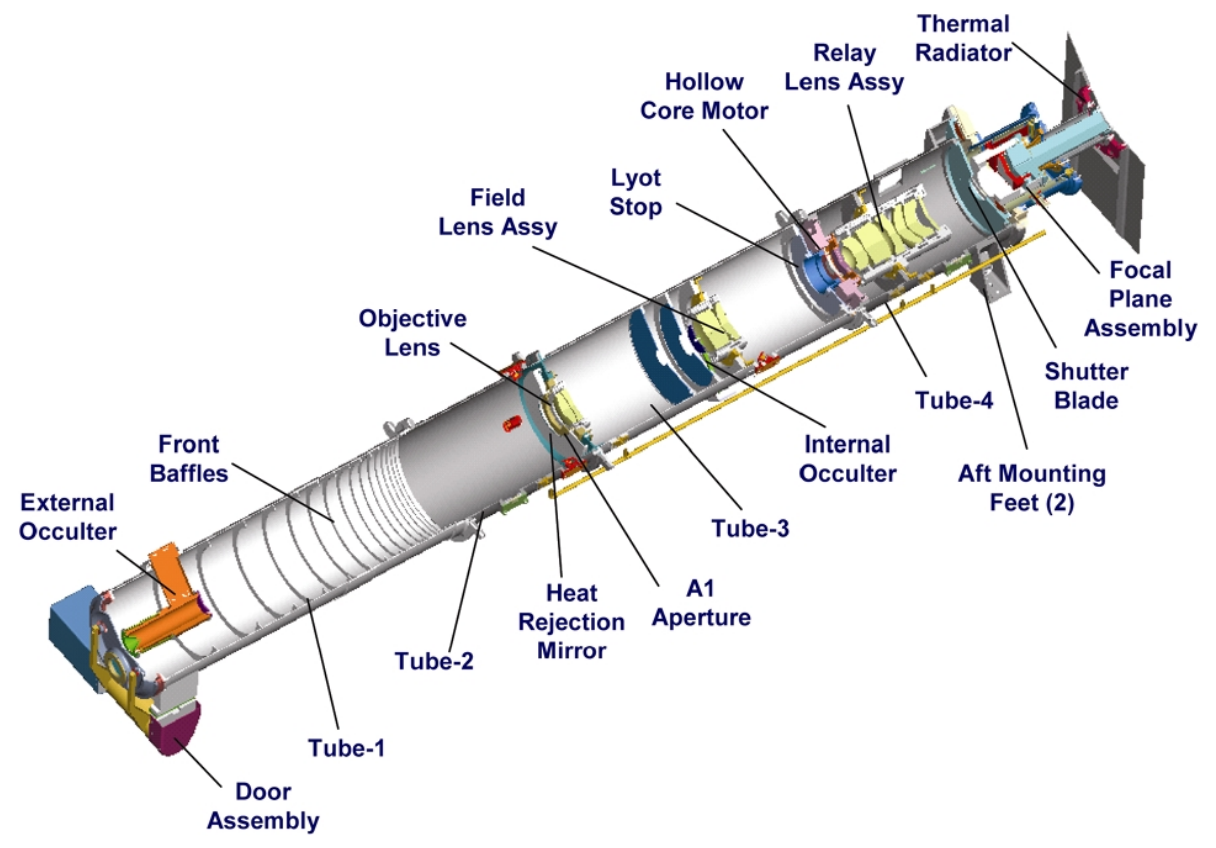

Fig. 25: Technical layout of the COR2 coronagraph on-board the STEREO spacecraft (Howard et al., 2008). The solar radiation traverses the coronagraph beginning at the first aperture (bottom left) and the external occulter blocking the direct solar radiation. After crossing several lens groups, aperture and occulter systems, an image of the polarised brightness is generated in the focal plane.

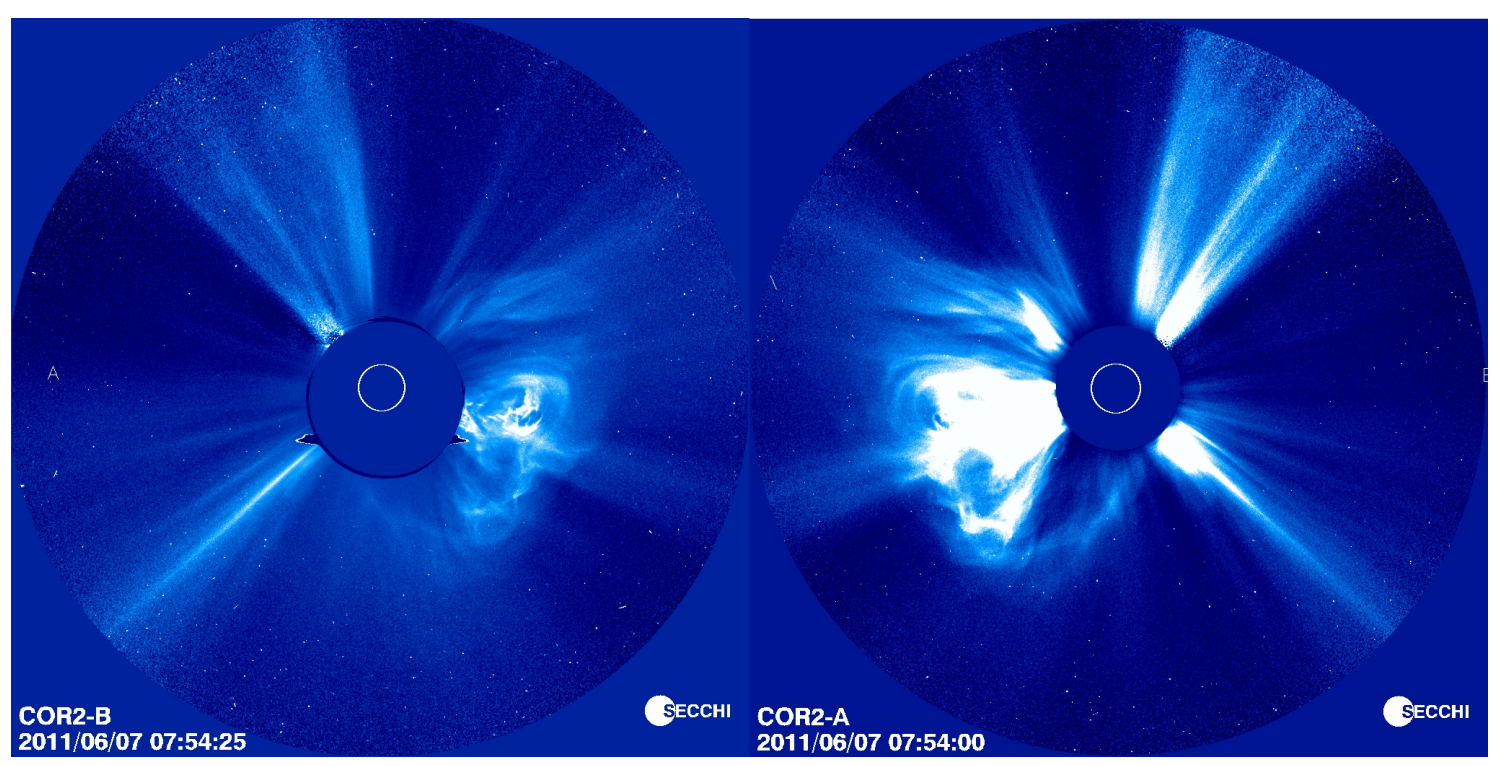

Fig. 26: Observations of the solar corona measured with STEREO/SECCHI/COR2-B (left) and -A (right) in white-light and visualised in false colour blue. The white-light appearance of a bright CME which was observed on October 11th, 2010 is visible in both images. From NRL, STEREO/SECCHI.

Further information about the COR2 telescope can be found in the SECCHI publication by Howard et al. (2008). 


\subsubsection{The Extreme UltraViolet Imager - EUVI}

The EUVI instrument on-board STEREO was developed to image the solar chromosphere and low corona in four emission lines. As a normal incidence EUV telescope it is based on the long lasting successful operating Extreme Ultraviolet Imaging telescope (EIT) on-board SOHO. The coronal plasma is fully ionised due to the high temperatures $\left(\sim 10^{6} \mathrm{~K}\right)$ of the corona. Highly ionised elements emit radiation at specific wavelengths which are measured as emission lines. For example Fe IX at $1.3 \times 10^{6} \mathrm{~K}$ emits EUV radiation at $17.1 \mathrm{~nm}$ and the emission line of $\mathrm{He} \mathrm{II}(80000 \mathrm{~K})$ is found at $30.4 \mathrm{~nm}$. The measurement of the emission lines at different wavelengths corresponds to different temperature profiles of the solar corona. The EUVI telescope observes the solar disk at $17.1 \mathrm{~nm}$ (Fe IX), $19.5 \mathrm{~nm}$ (Fe XII), $28.4 \mathrm{~nm}$ (Fe XV) and $30.4 \mathrm{~nm}$ (He II).

With a circular full Sun field of view to \pm 1.7 solar radii the EUVI telescope allows to investigate the low coronal source regions of CMEs. The EUVI images provide information about the source regions of an associated erupting CME. To distinguish the images in the aforementioned wavelengths they are colour coded as shown for EUVI-A in Figure 27. The EUVI image at $304 \AA$ (red) at the bottom right shows a large prominence eruption. This eruption is associated with the CME shown in Figure 26.

The main properties of all SECCHI telescopes are summarised in Table 4. Further detailed information about the EUVI telescope are published in Howard et al. (2008).

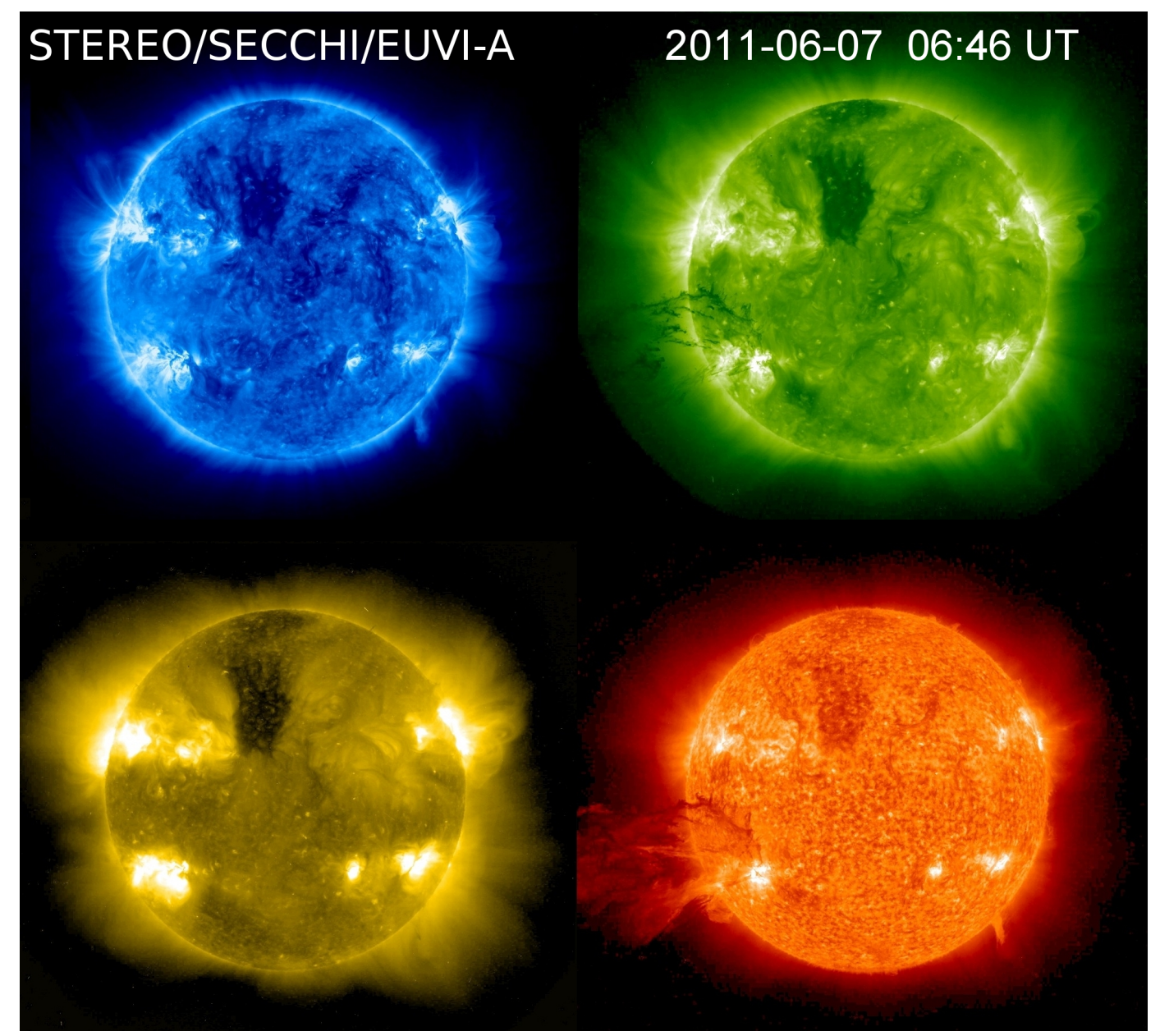

Fig. 27: Observations of the solar disk measured with STEREO/SECCHI/EUVI-A in four wavelengths at $171 \AA$ (blue), $195 \AA$ (green), $284 \AA$ (yellow) and $304 \AA$ (red). A prominence eruption is visible at 195 and $304 \AA$ which is associated with the CME observed on June 7th, 2011, see also Figure 26. From NASA STEREO/SECCHI and GSFC. 


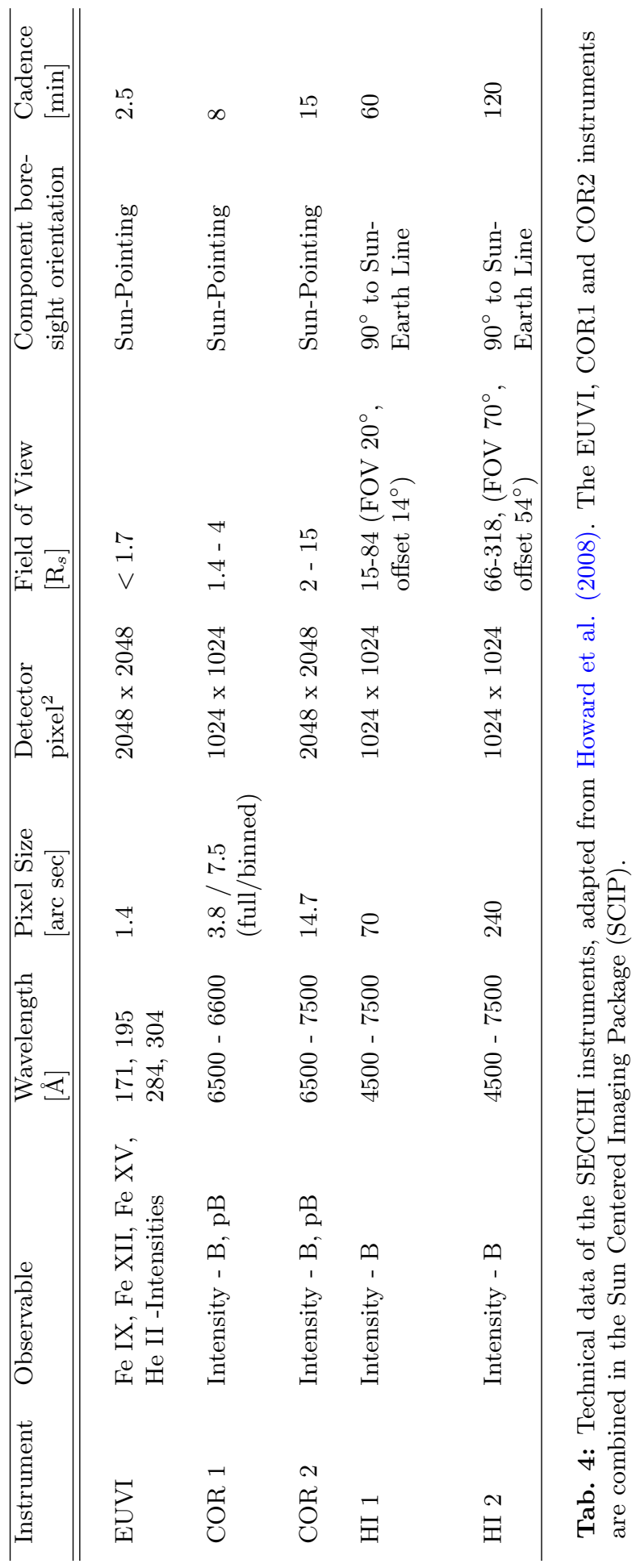





\section{Morphological Classification of CMEs}

In the past CMEs were observed and investigated with coronagraphs of several spacecraft missions. The white-light appearances of CMEs, visible in plane-of-sky observations, were described in different ways in this process. Already at the time of the Skylab mission in 1973/74 it was found from observations with the S-052 'White Light Coronagraph' that CMEs appear in different shapes and structures. The morphologies of $77 \mathrm{CMEs}$ which were identified during this mission were described as loops, clouds, streamer injections, filled bottles, rays or streamer separations (Munro et al., 1979). A few years later the Solar Maximum Mission (SMM) revealed new insights of the CME's visual nature. Based on a set of 65 CMEs observed with the SMM coronagraph the CME appearances were discussed as loops or bubbles, clouds and radial 'tongues' in a publication by Wagner (1984). Illing and Hundhausen (1985) deduced from coronagraph observations, taken in August 1980, the three-part structure for CMEs as described in Section 2.3. CMEs showing a three-part structure were also found in observations of the SOHO/LASCO coronagraphs (Gopalswamy, 2006; Chen, 2011).

All of these missions allowed to observe CMEs from one vantage point in space and to study their two-dimensional morphology and shape with plane-of-sky observations. The scientific questions in this context are: How does a CME look like in 2-D? And which morphologies can be observed in coronagraph images?

In this chapter, the two-dimensional CME morphologies are determined from plane-of-sky coronagraph observations of the current STEREO mission. For this purpose the STEREO/SECCHI/ COR2 coronagraph data were inspected for the time period from January 2007 until December 2011, in order to analyse CMEs occuring during the solar minimum as well as during the phase of increasing solar activity. The examination of the COR2 synoptic movies ${ }^{10}$ revealed 1071 CMEs occuring during this time period. These detected CMEs are registered in an overall CME list ${ }^{11}$. From this list a 'Best-of' CME list was established based on the visual appearance of the CME's white-light structure in the coronagraph images. The 'Best-of' list (Appendix A.4) comprises 241 CMEs which appear clear in brightness and structure in the COR2 coronagraph field of view. These CMEs are analysed in detail in this thesis.

As a first step the two-dimensional morphologies of these CMEs are examined in this chapter. Afterwards (Section 5.4 and 7) the three-dimensional structure of those CMEs is analysed with the Graduated Cylindrical Shell model which is applied to the stereoscopic observations from STEREO. During the initial visual inspection of the STEREO/SECCHI/COR2 synoptic movies several types of CME shapes were discovered. Based on the CME's visual appearance in structure and shape $10 \mathrm{CME}$ classes were defined. These classes are introduced in the following section. Subsequently, the 'Best-of' CMEs were categorised according to these CME class definitions.

\subsection{Description of CME Classes}

The CMEs' white-light appearances in the COR2 observations were classified into 10 classes based on the eye judged CME's white-light structure and shape. Below, the CME classes are introduced with their CME class name, a short description about the visual characteristics and a sample CME image for each CME class. It should be noted, that a classification of the visual appearance of CMEs is per se to a certain extent subjective.

\section{CME Class 1 - Halo CME:}

This class comprises all CMEs appearing as Halo within the coronagraph's field of view (FOV). A CME with an expansion in angular width of $360^{\circ}$ or approximated fulfills the criteria. As an example the 'Best-of' CME No 891 observed on September, 22nd 2011 is shown in Figure 28. 17 coronal mass ejections out of 241 'Best-of' events were classified as Halo CME. No differentiation was made between a front side or back side Halo CME.

\footnotetext{
${ }^{10}$ COR2 synoptic movies: http://secchi.nrl.navy.mil/sccmovies/

${ }^{11}$ The overall CME list is accessible at: www. affects-fp7.eu/cme-database
} 


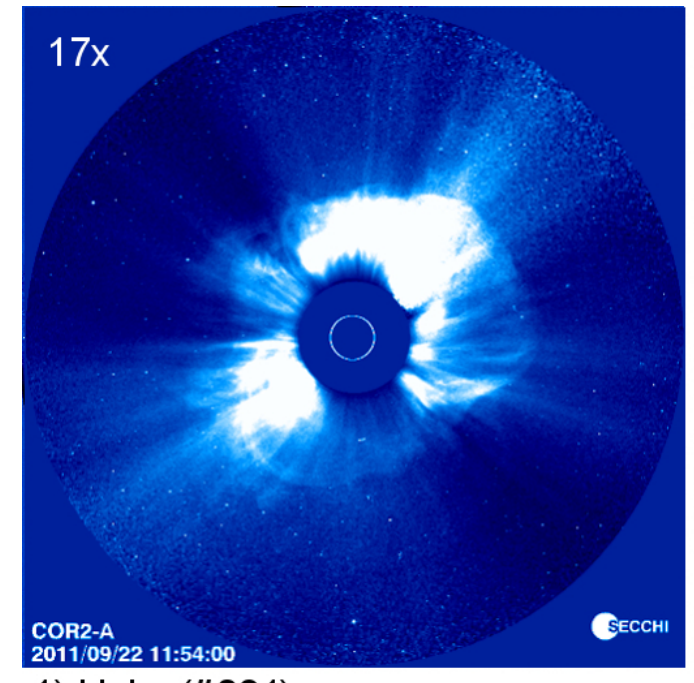

1) Halo (\#891)

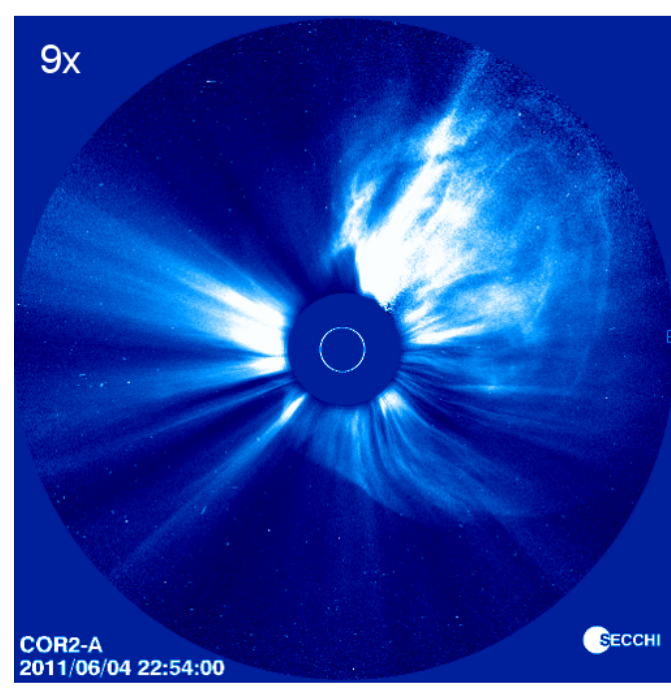

3) part. Halo (\#755)

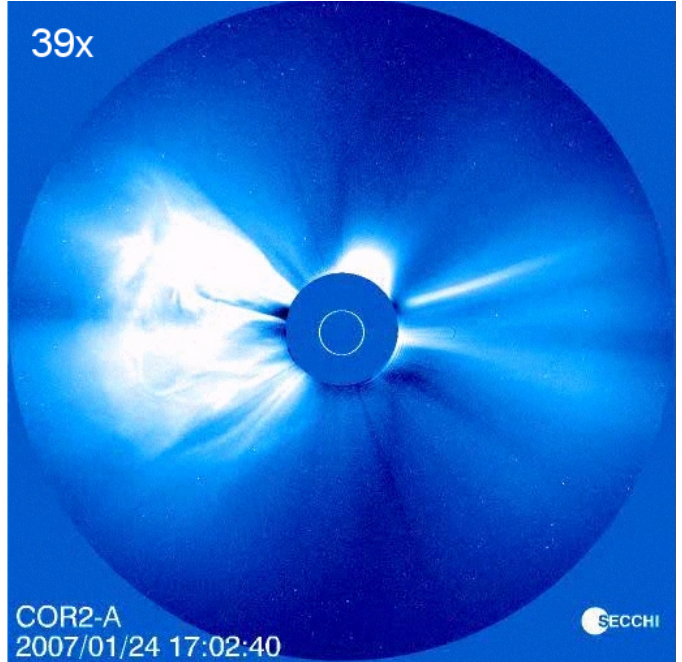

2) diffuse CME (\#5)

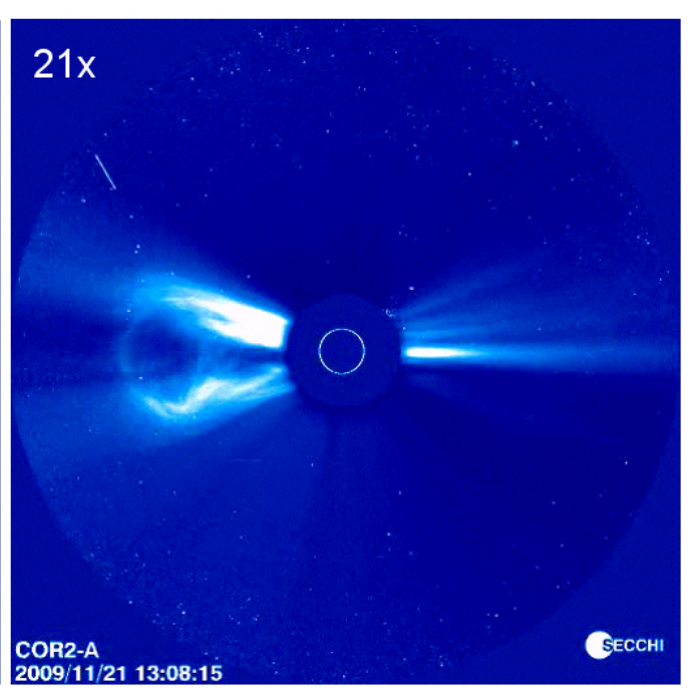

4) Ring-CME (\#299)

Fig. 28: The CMEs are divided into CME classes based on the structure and shape of their white-light appearance. These are the CME classes: 1) Halo CME, 2) diffuse CME, 3) partial Halo CME and 4) 'Ring'-CME. The population of these classes related to the set of 241 'Best-of' CMEs is given in white numbers. The numbers in parenthesis for each CME refer to the CME numbers from the 'Best-of' CME list, see Appendix A.4.

\section{CME Class 2 - diffuse CME:}

CMEs with no clear distinguishable structure or shape compose this class. The leading edge of these CMEs is not clearly defined and the inner part of these CMEs is unstructured and shows partly a swirled appearance. There were 39 events found and one of them is shown in Figure 28 on the top right.

\section{CME Class 3 - partial Halo CME:}

Similar to the first class this type of CMEs exhibits a large angular width typically larger than $180^{\circ}$. The involved material surrounds the occulter not completely but to a large extent. 9 out of $241 \mathrm{CMEs}$ are rated as partial Halo CME with one example shown in Figure 28. 


\section{CME Class 4 - 'Ring' CME:}

This type of CME exhibits a shape consisting of a cone like bottom part and an upper part reminiscent to a faint ring or circle. The bottom part is characterised by a dark void between the two flanks. For these 21 cases the angular width in plane of sky is typically smaller than $45^{\circ}$.

\section{CME Class 5 - 'jet-like' CME:}

'Jet-like' CMEs appear with an unclear unstructured shape in brightness and have a small angular width $\leq 45^{\circ}$ in the plane of sky. Five of them were counted in the 'Best-of' list and one example is shown in Figure 29.

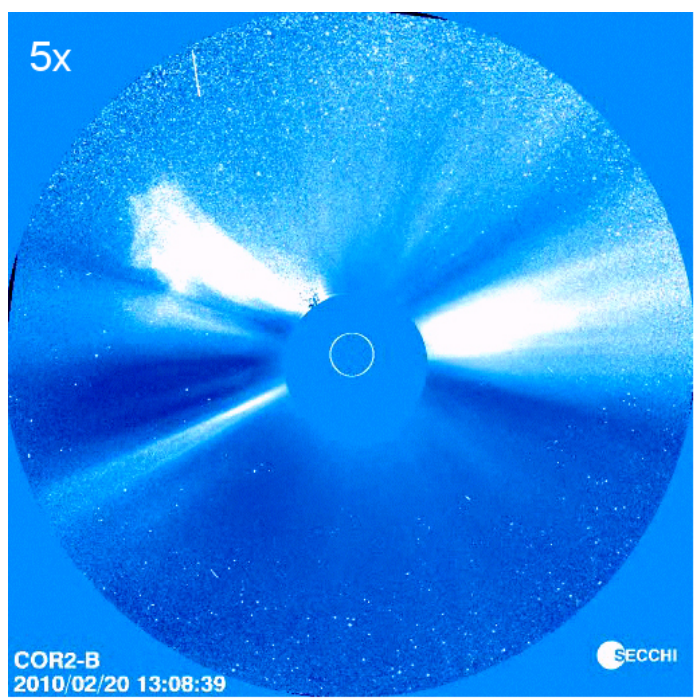

5) Jet-like CME (\#341)

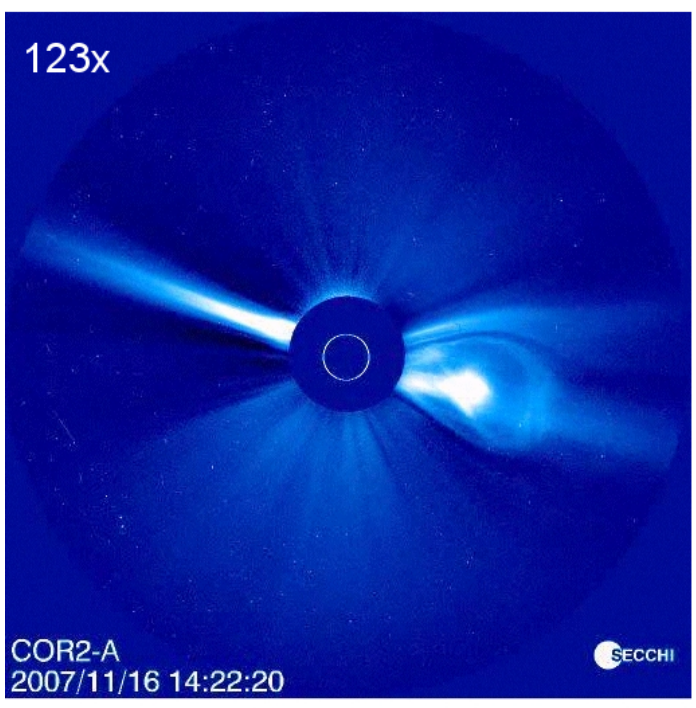

7) classical CME (\#86)

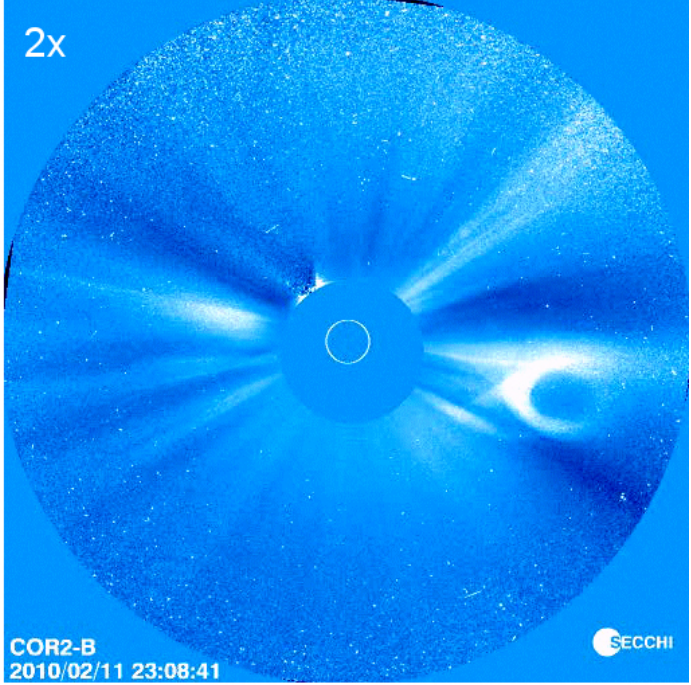

6) 'Fish'-CME (\#333)

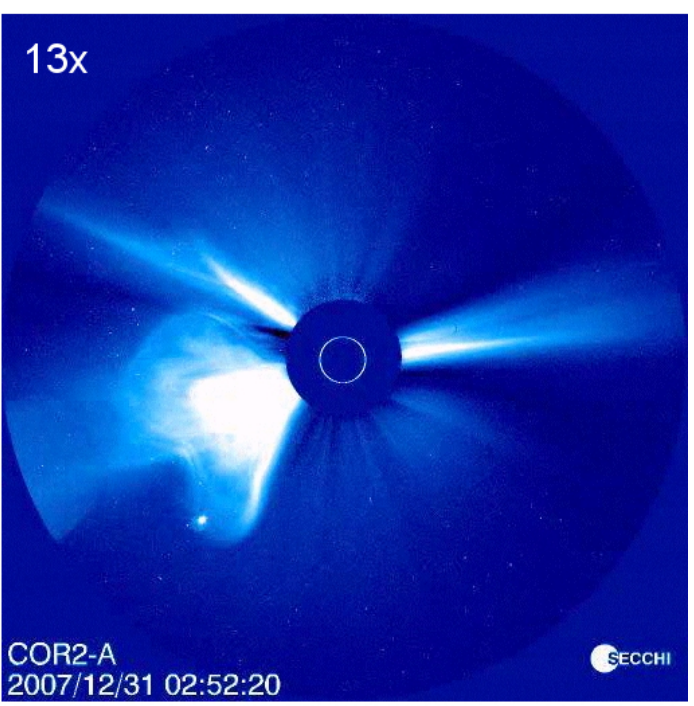

8) distorted CME (\#92)

Fig. 29: Examples of CMEs of their classes: 5) 'jet-like' CME, 6) 'Fish-like' CME, 7) classical CME and 8) distorted CME. The population of the classes related to the set of 241 'Best-of' CMEs is given in white numbers. The numbers in parenthesis for each CME refer to the CME numbers from the 'Best-of' CME list, see Appendix A.4. 


\section{CME Class 6 - 'Fish-like' CME:}

CMEs rated as 'Fish-like' CME exhibit a structure analogous to the ' $\propto$ ' symbol $^{12}$. The upper part of the CME (Figure 29 top right) is formed like a ring. In this Figure the CME shows a faint leading edge whereas the trailing part of the ring is bright with two crossing lines beneath. With this characteristical crossing lines the structure is reminiscent to a greek symbol also known as 'sign of the fish'. In the 'Best-of' CME list only two of the described 'Fish'-CMEs were observed and one of them is shown in Figure 29.

\section{CME Class 7 - classical CME:}

This type of CME constitutes the largest amount of classified CMEs with 123 events. These CMEs can be described with the 'three-part structure' as published by Illing and Hundhausen (1985) and the Bothmer-Cremades scheme for CMEs as presented in Section 2.3 (Figure 7 and 11, respectively). These CMEs exhibit a clear distinguishable structure and shape. The CME's leading edge is clearly visible pronounced with a curvature which is not distorted.

\section{CME Class 8 - distorted CME:}

CMEs of this class are characterised by a clear leading edge with a distortion. The CME shown in Figure 29 (bottom right) exhibits a front with a concave formed part. During the observation of a CME's outward propagation two different cases can occur. The first consists in a CME which shows a small distorted leading edge at the beginning of the observation near the solar disk. As time passes the CME moves away from Sun and the initial distortion of the leading edge increases. In this case the CME propagates with a self similar expansion. This applies to the presented CME. The second case constitutes a CME which is observed without a distortion at the beginning. But during its propagation away from Sun a distortion at the leading edge evolves and enlarges which indicates a non self similar expansion of the CME's front. Both cases were observed and included in this CME class. In sum 13 coronal mass ejections show a distorted leading edge.

\section{CME Class 9 - 'Laurel wreath' CME:}

This type of CME attracts attention because at the middle of the leading edge no bright front is visible which would complete a ring structure similar to CME class 4 . This shape is reminiscent to a laurel wreath and was observed for 7 CMEs. The CME's bottom part shows a thin (distorted) cone-like shape. An example is given in Figure 30.

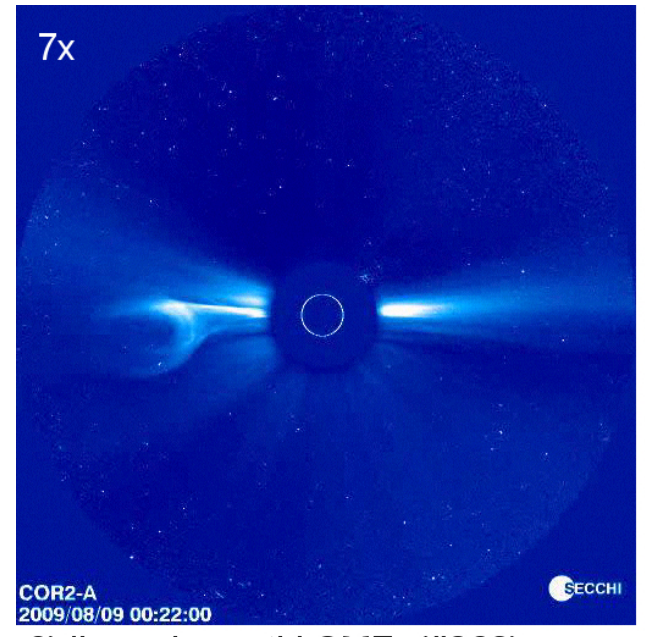

9) 'Laurel wreath' CME (\#263)

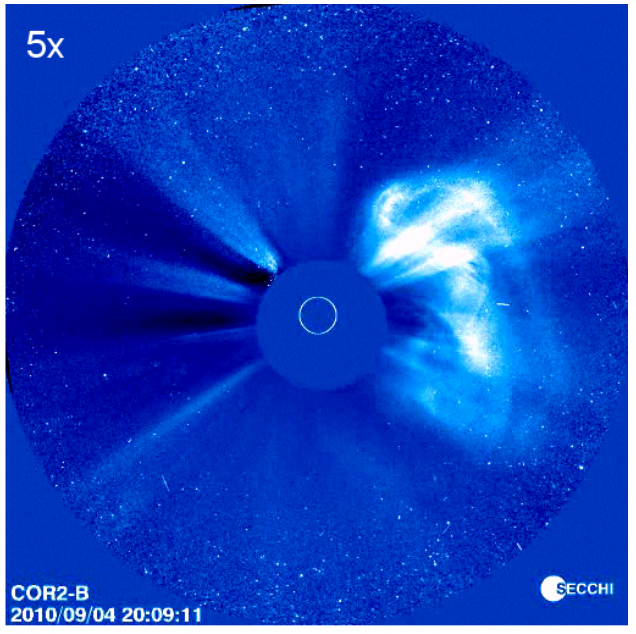

10) non-rated CME (\#494)

Fig. 30: Examples of CMEs which represent the following classes: 9) 'Laurel wreath' like CME and 10) non-rated CME. The population of the classes related to 241 Best-of CMEs is given in white numbers. The CME numbers in parenthesis refer to the numbers from the 'Best-of' CME list, see Appendix A.4.

\footnotetext{
12 'ichthys' from the greek 'ikhthys', is a symbol also known as "sign of the fish".
} 


\section{CME Class 10 - non-rated CME:}

Five of the inspected CMEs show a bright structure, but these different structures can not be assigned to a unique scheme for classification. Furthermore, these CMEs don't feature the characteristics of the before introduced CME types. Therefore these events were uncategorised (non-rated).

As we have seen CMEs occur with large variations in their two-dimensional white-light appearance. This leads to the assumption of several morphologies for CMEs which are grouped in 10 CME classes.

\subsection{Comparison with CME Survey by Howard (1985)}

In 1985 Howard et al. performed a large CME survey based on Solwind observations and analysed systematically a large data set of 998 CMEs in terms of different structural classes. The authors analysed the CMEs in terms of brightness, shape and structure and CME properties like central latitude, latitudinal spans, speed, mass, kinetic energy and occurrence rate. In this section Howard's study is introduced and their structural classes are contrasted with the previously introduced CME classes from this thesis. Furthermore the results from the comparison will be discussed.

\subsubsection{Introduction}

Howard et al. identified 998 CMEs in images of the Solwind coronagraph during the time period from 1979 to 1981. The plane-of-sky CME observations were subdivided in three 'categories of importance' for the CME's white-light appearance. Based on visual inspection of the coronal difference images CMEs were categorised as 'major' CMEs if they exhibited doubtless a large and bright white-light appearance. CMEs with a small and faint appearance were grouped as 'minor' CMEs. The CME events which could not subdivided beyond doubt in one of these two categories are rated as 'questionable'. In addition, ten structural classes were defined based on the CME's shape visible in the coronagraph images. All CMEs were ranked in one of these classes. The CMEs that occurred during the maximum of solar cycle 21 were analysed in relation to their structural classes, category in brightness, central latitude, CME mass, kinetic energy and CME occurrence rate. The obtained CME properties are affected by projection effects. In contrast the presented CME survey in this thesis covers the time period from 2007 to 2011 with 1071 identified CMEs whereof 241 CMEs are rated as 'Best-of' CMEs comparable with the 240 'major' CMEs from Howards event list. The mentioned five years of solar cycle 24 cover the phase of minimum in solar activity and rising solar activity to the end of 2011. The technological observation conditions are completely different with the state-of-the-art STEREO twin observatory compared to the single Solwind spacecraft located at a 96-min orbit at Earth. These differences are compared to each other in Table 5. Further information about the Solwind mission and its white-light coronagraph can be found in the publications by Michels et al. (1980) and Sheeley et al. (1980).

\subsubsection{Structural Classes}

During his investigations of the difference images for the 998 identified CMEs Howard defined ten structural classes based on the CME's visual appearance in shape and structure. These classes are now presented with their comparatively contrasted CME classes of this thesis. 


\begin{tabular}{|c|c|}
\hline \multicolumn{2}{|c|}{ Description of both CME surveys: } \\
\hline 1979 - 1981 (3 years) & $2007-2011$ (5 years) \\
\hline around solar max. (1981) & solar min. to rising solar max. \\
\hline 998 CMEs identified and analysed & 1070 CMEs identified \\
\hline with 240 'major' CMEs & 241 'Best-of' CMEs analysed \\
\hline $\begin{array}{l}\text { Brightness category: } \\
\text { major, questionable, minor }\end{array}$ & $\begin{array}{l}\text { 'Best-of' CMEs correspond } \\
\text { to 'major' CMEs }\end{array}$ \\
\hline $\begin{array}{l}\text { Solwind coronagraph characteristics } \\
\text { ('first generation' coronagraph) }\end{array}$ & $\begin{array}{l}\text { STEREO/SECCHI/COR-2 } \\
\text { ('third generation' coronagraph) }\end{array}$ \\
\hline Field of view: $2.5-10 r_{\odot}$ & $2-15 r_{\odot}$ \\
\hline Pixel size: 1.25 arc min & 14.7 arc sec \\
\hline Cadence: $10 \mathrm{~min}$ & $15 \min$ \\
\hline 96-min Orbit at Earth & $\begin{array}{l}\text { Earth-like orbit, in front of } \\
\text { and trailing to Earth }\end{array}$ \\
\hline Difference images & $\begin{array}{l}\text { Difference images from } \\
\text { total brightness images }\end{array}$ \\
\hline $\begin{array}{l}\text { Analysis based on plane-of-sky } \\
\text { observations }\end{array}$ & $\begin{array}{l}\text { Results from } 3 \text {-D GCS analyses } \\
\text { without projection effects }\end{array}$ \\
\hline
\end{tabular}

Tab. 5: Description of both CME surveys performed by Howard et al. (1985) and from this thesis.

\section{'Loop' CME vs. 'Ring' CME:}

The class of 'Loop' CMEs is defined by two legs connected with a curved front. The leading and trailing edge is clearly visible. Behind the trailing edge and between the legs is located a dark void which results in the loop structure. The left frame in Figure 31 shows such a 'Loop' CME observed on September 6th, 1980 with the Solwind coronagraph. The CME class which would match most to the 'Loop' CME is the 'Ring' CME. An example is given in the center frame which shows the 'Ring' CME at an early point of its propagation outwards. The visual appearance matches the 'Loop' CME very well. Note, that the northern part (upper leg) of the 'Ring' CME is superimposed by a small streamer at this time. Four hours later (right frame in Figure 31) the 'Ring' CME
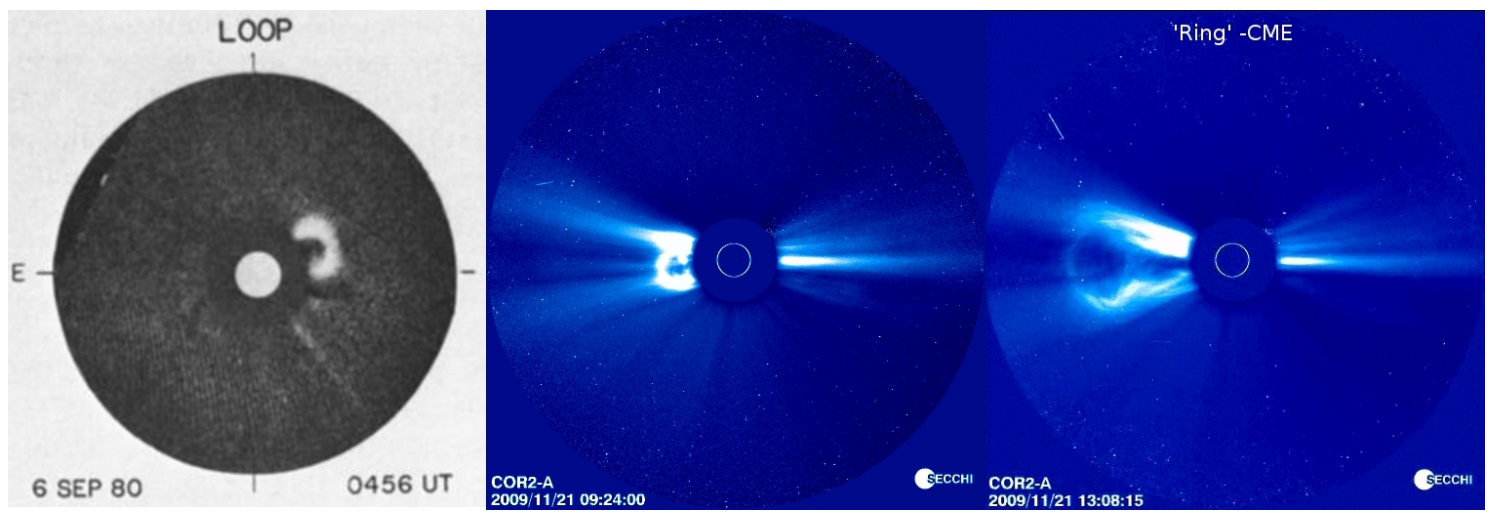

Fig. 31: The CME observed on September 6th, 1980 by the Solwind coronagraph is shown on the left, from Howard et al. (1985). This 'Loop' -CME is compared to the 'Ring'-CME observed on November 21st, 2009 with STEREO/SECCHI/COR2-A and later on full expanded in the right image.

evolved its shape and the conical shaped rear part becomes visible. The front part changed with a fainter leading edge in which the legs are now connected with the leading and trailing curvature 
composing a ring structure. Hence the 'Ring' CME only matches in its early phase of evolution with the 'Loop' CME.

\section{Curved Front CME vs. classical CME:}

The 'curved front' CME is characterised by a distinct leading edge followed by a bright large region without a trailing edge as seen for 'Loop' CMEs. Such CMEs as shown in the left frame in Figure 32 agree best with the class of 'classical' CMEs presented in the center frame. Both CME classes exhibit the same characteristics by definition and visual appearance. Because Howard made no differentiation or limitation in angular width for the 'curved front' CMEs also CMEs from the 'partial Halo' class of this thesis would match like the one shown in the right frame of Figure 32. But depending on the angular width of 'partial Halo' CMEs it is questionable if also 'partial Halo' CMEs with an angular width larger than $180^{\circ}$ can be compared sensibly to 'curved front' CMEs. Note the decrease in brightness due to the coronagraph's first polariser ring at about $4 \mathrm{r}_{\odot}$ for the Solwind observation in the left frame.
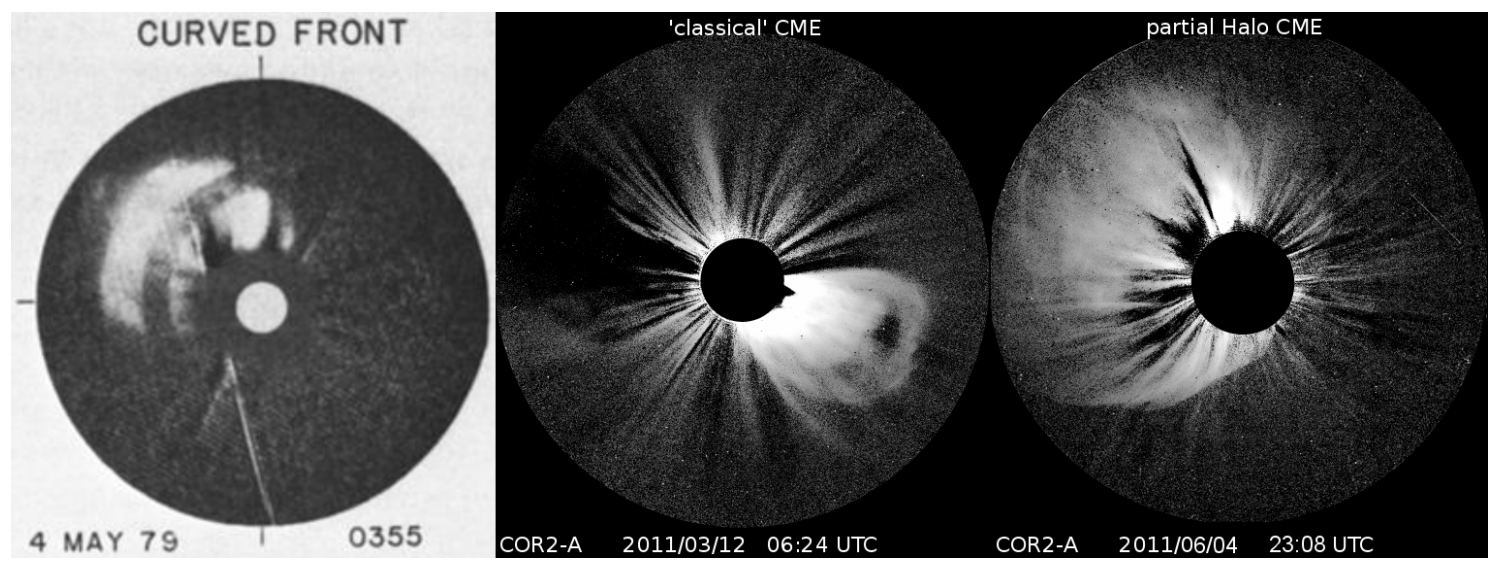

Fig. 32: The CME observed on May 4th, 1979 with the Solwind coronagraph is presented on the left, from Howard et al. (1985). Note the decrease in brightness due to the coronagraph's first polariser ring at about $4 \mathrm{r}_{\odot}$. This 'curved front' CME is contrasted to the 'classical' CME shown in the center and to the 'partial Halo' CME on the right image, both observed with STEREO/SECCHI/COR2 in March and June of 2011, respectively.

Halo CME (1985) and Halo CME (2015):

The Halo CME class from Howard et al. and from this thesis agree by definition one-to-one for CMEs which show a bright appearance all around the solar disc. For both classes no differentiation was made between disc centered symmetrical Halo CMEs and non disc centered asymmetric Halo CMEs. Strictly speaking only the former propagate towards or away from the observer along the line of sight. The latter exhibit a certain lateral component of movement relative to the observer. An example for a Halo CME observed on April 18th, 1981 with the Solwind coronagraph (left frame) is shown in Figure 33 compared with a CME observed on September 22nd, 2011 by STEREO/SECCHI/COR2-A. Please note again the decrease in brightness in the left coronagraph observation due to the two polariser rings at about 4 and $8 \mathrm{r}_{\odot}$.

\section{Spike CME vs. 'Jet-like' CME:}

The Spike CME class from Howard represent narrow jet-like CMEs which can be compared oneto-one with the 'jet-like' CME class from this thesis. It has been observed that Spike or 'jet-like' CMEs sometimes but not always propagate outwards along a streamer (Howard et al., 1985). A Spike CME observed on March 21st, 1981 with the Solwind coronagraph is presented in Figure 34 (left frame) and for comparison a 'jet-like' CME from February 20th, 2010 is shown in the 


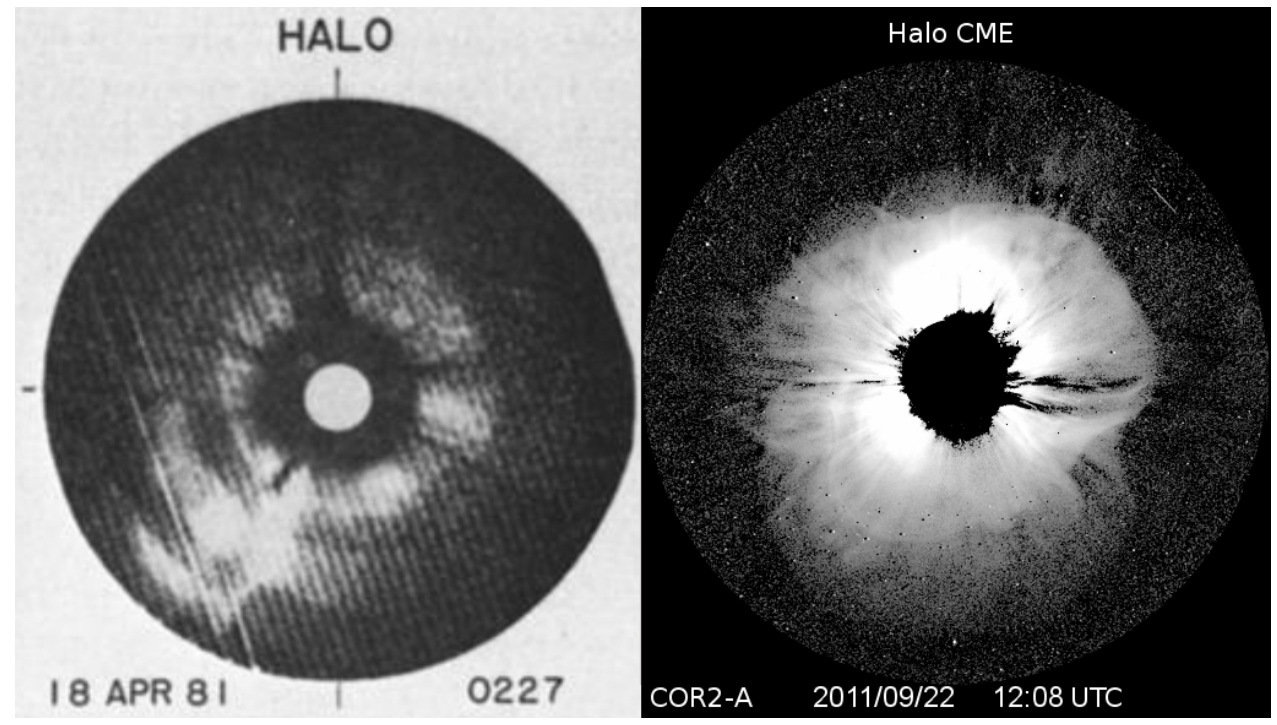

Fig. 33: A Halo CME observed on April 18th, 1981 with the Solwind coronagraph is shown on the left, from Howard et al. (1985). Note the decrease in brightness due to the two coronagraph's polariser rings at about 4 and $8 r_{\odot}$. This one is compared with the Halo CME observed on September 22nd, 2011 with STEREO/SECCHI/COR2-A.

STEREO/SECCHI/COR2-B image (right frame).
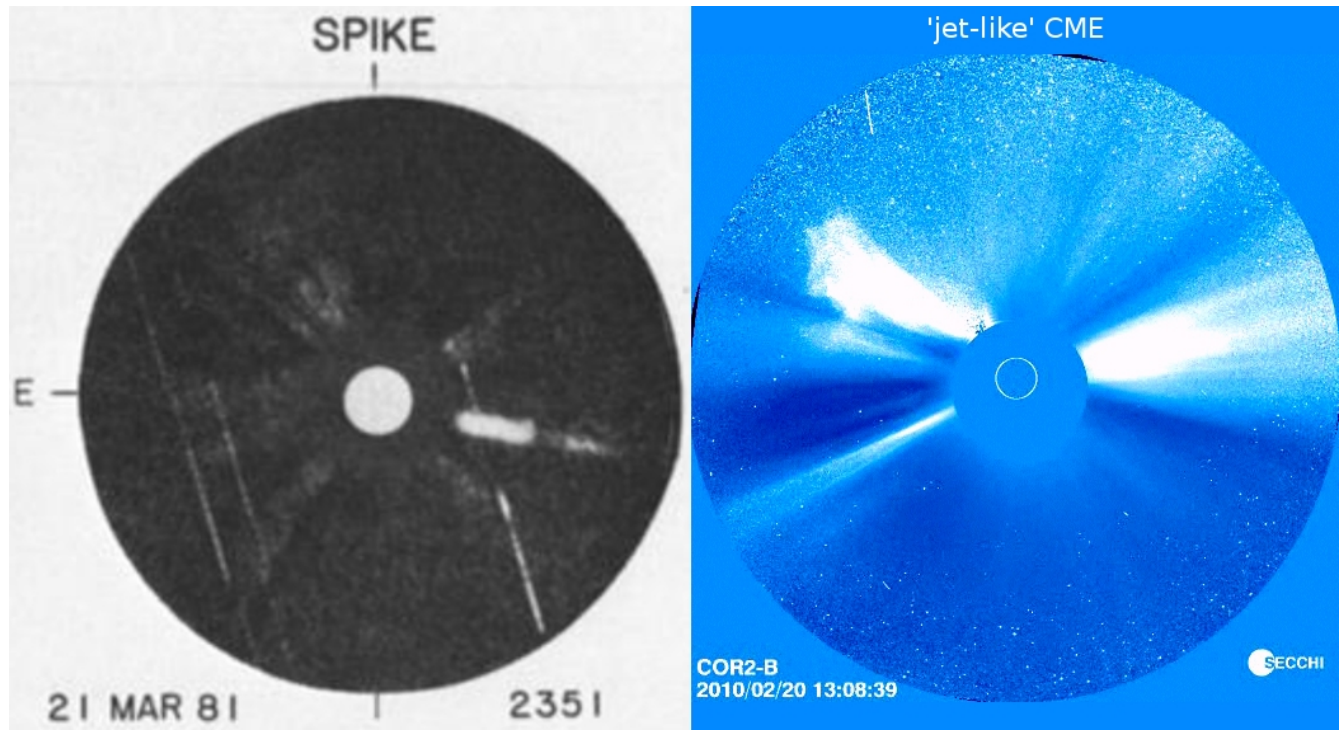

Fig. 34: A CME observed on March 21st, 1981 with the Solwind coronagraph is shown in the left frame, from Howard et al. (1985). This Spike CME is compared to the 'jet-like' CME observed with STEREO/SECCHI/COR2-B on February 20th, 2010. 


\section{Double Spike CME vs. 'Laurel Wreath'-like CME:}

CMEs categorised as 'Double Spike' CME in the Solwind observations exhibit two bright Spikes or legs separated by a dark region between. These CMEs don't show a leading edge at the front which would connect both Spikes. An example for a 'double spike' CME is shown in the left image of Figure 35. A 'Streamer Blowout' CME observed on May 29th, 1980 is presented in the center frame. This type of CME occurs at the beginning as bright streamer which enlarges and broadens over a time period of several hours up to one day with material ejected outwards along its radial orientation (Howard et al., 1985). In its later evolution further material is expelled on both sides of the streamer and the center part fades out in brightness. This phase is shown in the center frame of Figure 35 and looks similar to the white-light appearance of the 'double spike' CME. Compared to the CME classes from this thesis no corresponding class is found. The closest matching class constitutes the 'Laurel wreath' -like CME. Such a CME is shown in the right frame of the Figure. Its front part consists also of two open legs with a dark void between and a missing CME front. But in the rear part both legs merge to one stripe. Therefore the match with one of the first two CME classes appears insufficient.
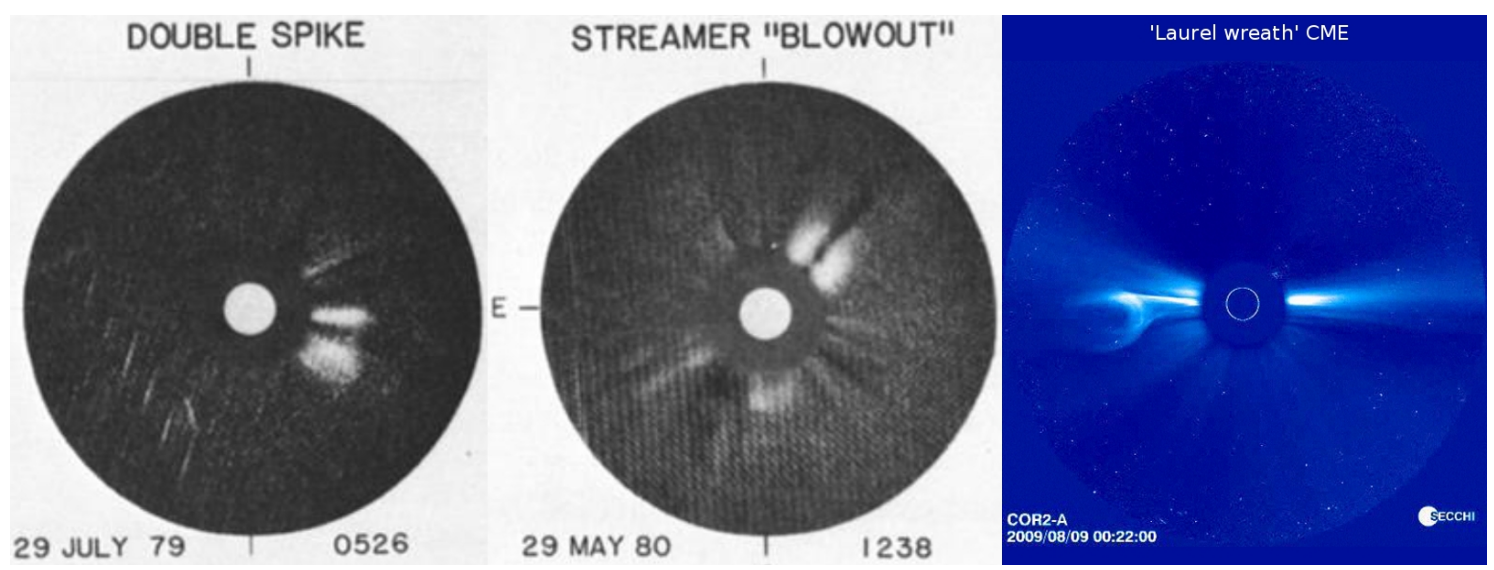

Fig. 35: The 'Double Spike' CME observed on July 29th, 1979 with the Solwind coronagraph is presented on the left, from Howard et al. (1985). In addition a CME detected on May 29th, 1980 which is categorised as 'Streamer Blowout' CME is displayed in the middle. The 'Laurel wreath' -like CME on the right corresponds only with some restrictions to these both CME classes.

\section{Multiple Spike CME vs. distorted CME:}

CMEs which exhibit a white-light appearance consisting of more than two spikes and therefore showing an irregular CME front were classified as 'multiple spike' CME by Howard. A CME with such a characteristic is shown in Figure 36 (left frame). Again the polariser ring of the coronagraph causes a decrease in brightness at about $4 \mathrm{r}_{\odot}$ so that unfortunately the tips of the spikes are covered. Their estimated positions are indicated by red arrows. A distorted CME with its deformed leading edge is presented for comparison in the right frame of Figure 36. The foremost parts of the distorted leading edge are marked with red arrows indicating possible similarities to the front part of the multiple spike CME. Even though the distorted CME doesn't show a multiple spike structure both types of CME can be compared to a certain extent. The front part of the multiple spike CME corresponds potentially more to a distorted leading edge than to several spikes forming the front. But this cannot be verified with the available coronagraph images from the publication by Howard et al. (1985). 


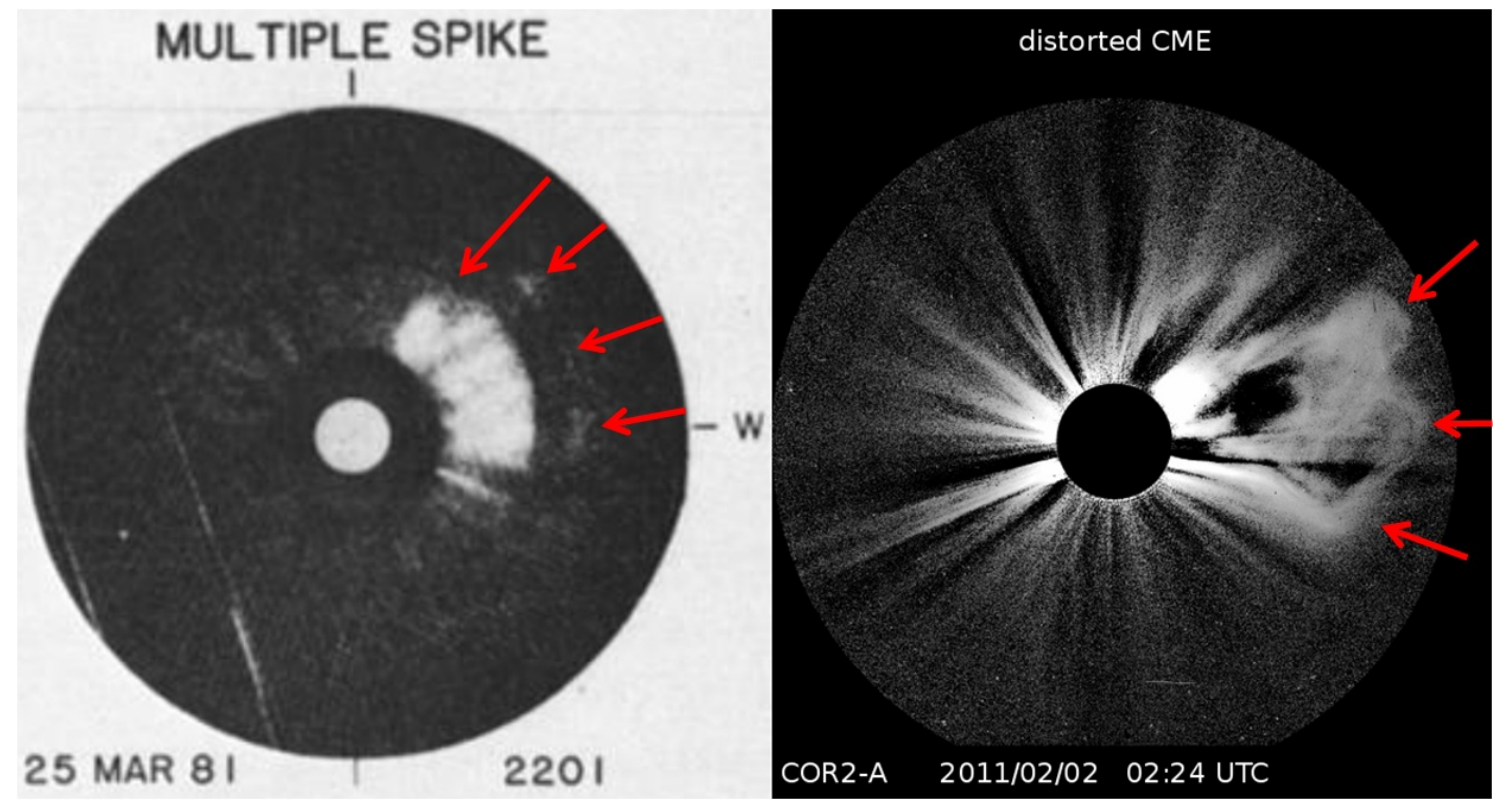

Fig. 36: A 'Multiple Spike' CME observed on March 25th, 1981 with the Solwind coronagraph is shown on the left, from Howard et al. (1985). Note the decrease in brightness due to the first coronagraph's polariser ring at about $4 \mathrm{r}_{\odot}$. Unfortunately this polariser ring covers three spikes indicated with red arrows. The tips of the multiple spikes potentially correspond to a distorted leading edge similar to the CME shown on the right.

\section{Complex CME vs. diffuse CME:}

Howard et al. defined the class of 'Fan' CMEs as those events which appeared with only a slight or no internal structure. These CMEs show no distinct clear leading edge but exhibit straight borders on the left and right side. One of these CMEs was observed on June 10th, 1979 (left frame in Figure 37). A CME revealing a complex shape and structure which could not be allocated to one of the other classes is categorised as 'Complex' CME by Howard (center frame). Applying the definition of Howard's class of complex CMEs a diffuse CME could correspond to this one. In order to examine this a diffuse CME observed on January 24th, 2007 with the STEREO/SECCHI/COR2-A coronagraph is presented in the right Figure. This CME exhibits no distinct leading edge but a diffuse internal structure. A comparison with the complex CME shown in the center frame is not possible due to its very faint white-light appearance.

\section{'Other' CMEs:}

The tenth class of CMEs represents events whose shape could not be defined with the visual inspection of white-light coronagraph images. Also CMEs detected with a leading edge lying outside of the coronagraph's field of view are included in this category. This class would correspond to the non-rated CMEs of the CME classes of this thesis.

\subsubsection{Results from Comparison}

The comparison of the analysis results from both CME surveys by Howard and from this thesis is discussed in this section.

The CME study by Howard et al. extends over the time period from March 1979 until December 1981 around the maximum of solar activity in terms of monthly sunspot numbers. For this time of solar cycle 21 a CME occurrence rate of $1.8 \mathrm{CMEs}$ per day (54 CMEs/month) was found. This rate corresponds to the monthly CME rate measured with the STEREO coronagraphs since the second half of the year 2011. Since this point in time the monthly smoothed sunspot number increased 

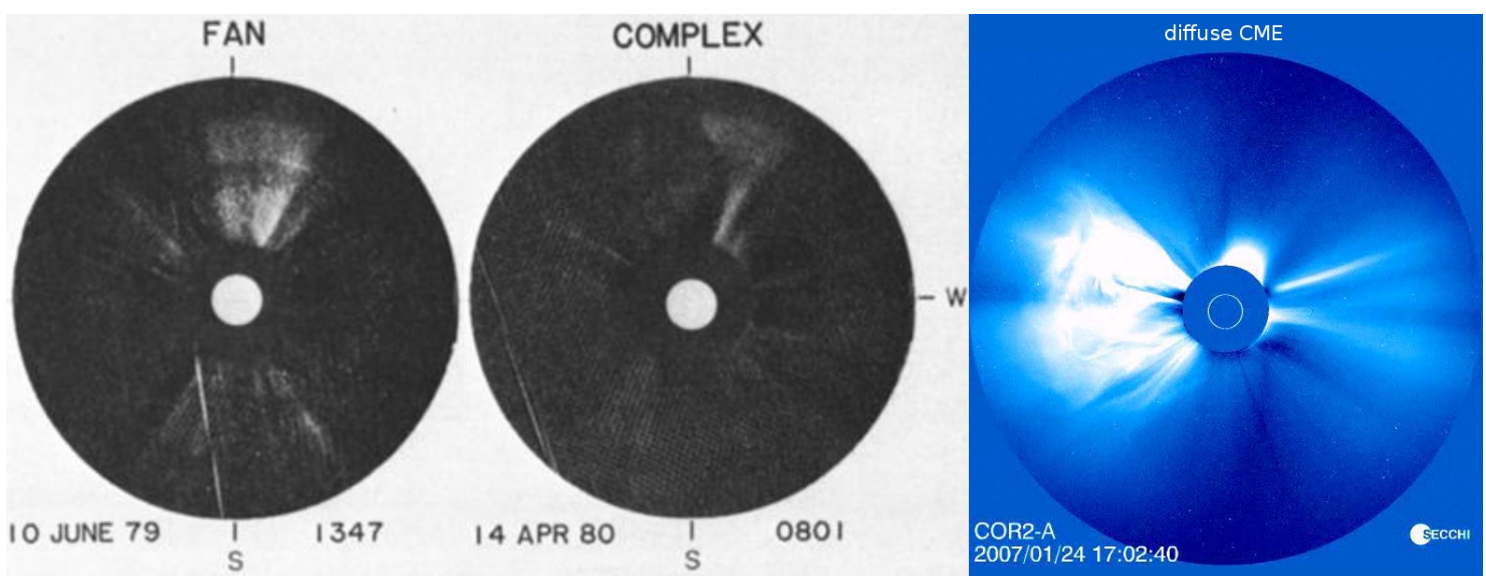

Fig. 37: A 'Fan' CME as introduced by Howard's CME class definition is presented in the left frame. A very faint 'Complex' CME observed on April 14th, 1980 with the Solwind coronagraph is shown in the middle (Howard et al., 1985). Based on the definition of a 'Complex' CME this one is compared to the diffuse CME shown on the right.

significantly as shown in Figure 62 a) in chapter 7. In contrast to results of this thesis and results from Gopalswamy in 2006 (for solar cycle 23, 1996 - 2006) Howard found no correlations between temporal variations of the CME occurrence rate and the rate of sunspots for time scales covering 7, 27 or 180 days. The CME classes defined by Howard et al. are listed in Table 6 together with the associated CME classes of this thesis.

\begin{tabular}{lll}
\hline Howard et al. (1985) & $\begin{array}{c}\text { Comparison of CME classes } \\
\text { this work }\end{array}$ & comment \\
\hline Loop CME (5) & Ring CME (21) & $\begin{array}{l}\text { match of Ring CME during } \\
\text { first phase of its evolution }\end{array}$ \\
\hline Curved Front CME (105) & $\begin{array}{l}\text { Classical CME (123) } \\
\text { Partial Halo CME (9) }\end{array}$ & $\begin{array}{l}\text { match } \\
\text { match for angular } \\
\text { width } \lesssim 180^{\circ}\end{array}$ \\
\hline Halo CME (8) & Halo CME (17) & $1: 1$ match \\
\hline Spike CME (0) & Jet-like CME (5) & $1: 1$ match \\
\hline Double Spike CME (11) & Laurel-wreath & $\begin{array}{l}\text { comparable with } \\
\text { restrictions }\end{array}$ \\
\hline Streamer Blowout (28) & - & no match \\
\hline Multiple Spike CME (34) & distorted CME (13) & $\begin{array}{l}\text { comparable with } \\
\text { restrictions }\end{array}$ \\
\hline Fan CME (4) & & no match \\
\hline Complex CME (32) & diffuse CME (39) & $\begin{array}{l}\text { comparable with } \\
\text { restrictions }\end{array}$ \\
\hline Other CMEs (13) & - & no match \\
\hline- & non-rated CME (5) & no match \\
\hline- & Fish CME (2) & no match \\
\hline 240 & 241 & all CMEs \\
\hline
\end{tabular}

Tab. 6: Comparison of the fractional distribution of CMEs for both CME class systems defined by Howard et al. (1985) and of this thesis. The number of CMEs for each class is specified in parentheses. 
The table summarises the comparison of CME classes from the previous section and presents the fractional distribution of CMEs in their classes. For the CME classes 'Curved Front', Halo CME and 'Spike CME' which match very well with their equivalents from the CME categories of this work the frequency in occurrence agrees also well with 105 'Curved Front' to 123 classical CMEs, 8 (Howard) to 17 (Bosman) Halo CMEs and zero 'Spike' to five 'Jet-like' CMEs. The populations of the 'Double Spike' and 'Complex' CMEs with their associated classes of this work also show similar numbers. 


\section{The Graduated Cylindrical Shell Model}

The observation of CMEs with a coronagraph reveals various morphologies in 2-D. Though these observations do not answer the question: Which three-dimensional structure do these CMEs have? In order to clarify this question in this chapter a CME model is discussed which allows to fit a CME's 3-D geometry from its visual appearance in the stereoscopic coronagraph images. This Graduated Cylindrical Shell (GCS) model describes the geometry of a CME in accordance with the flux rope model for CMEs (Section 2.3.4), Low (2001) and Chen (2011). The CME's 3-D geometry can be modeled with this GCS technique based on the coronagraph image data from STEREO/SECCHI/COR2. The 3-D CME parametrisation is presented in detail for six selected CMEs and the characteristics of CME modeling are illustrated. It will be shown how the GCS flux rope model looks like for these CMEs (Section 5.4). Finally, a short introduction about the identification and localisation of the CME's source region is given.

\subsection{Overview of CME Reconstruction Techniques}

In the past decades several techniques were used for reconstructing the solar corona or white-light structures like CMEs. Therefore, a brief overview about other CME reconstruction techniques is given in this section, before the Graduated Cylindrical Shell model is introduced.

(1) As inverse method the Solar Rotational Tomography (SRT) was applied to a sequence of SOHO/LASCO data by Frazin and Janzen (2002) to reconstruct the quiet solar corona. This technique requires a long continuous observation over half of a solar rotation to yield a large number of views of the solar corona which is assumed to stay stable during this time. This is not satisfied for a reconstruction of fast propagating CMEs. Even with the view from three spacecraft observatories (including SOHO) details of the CME's 3-D structure cannot be revealed sufficiently with conventional tomography.

(2) Triangulation methods are mostly used for generation of terrestrial topological maps. With this method a feature is localised from at least two different vantage points to derive its 3-D position in space. This technique was successfully applied to determine the CME's velocity and direction by Liewer et al. (2007, 2008). However, this approach does not support considerations about the electron density distribution of a physical object like a CME.

(3) Another possibility constitutes the polarisation method. The photospheric light is Thomson scattered by electrons of the observed CME and is polarised perpendicular to the Sun-electron line. The ratio of the polarised and unpolarised white-light measured by the coronagraphs depends on the distance between the electrons and the coronagraph's plane-of-sky (Billings, 1966; Thernisien et al., 2011). From analysis of the polarisation ratio the 3-D electron density distribution of the white-light structure can be derived. In the past line of sight effects from single point of view observations, signal to noise ratio and instrumental polarisation effects limited the application of this technique and in particular for fast propagating CMEs. In tracking fast moving features the polarisation information is blurred during the long time which is required to record three polarised images in sequence. But these constraints are reduced with the new STEREO/SECCHI coronagraphs providing fast cadence imaging with improved polarisation performance and operating synchronously from two separate vantage points. De Koning et al. (2009) and Moran et al. (2010) applied this technique successfully to reconstruct CMEs with STEREO coronagraph data.

(4) When the forward modeling technique is used, strong assumptions are made for the morphology of the object. An analytical function of a 3-D electron density distribution is introduced with parameters for adjusting the shape of the object. The challenge is to determine the parameter values which would best fit the model to the white-light observations. The choice of a preferred model allows to consider physical aspects of the object. In this case the Graduated Cylindrical Shell model is based on the approach of a magnetic flux rope morphology (Chen, 1996 and 2000). With the magnetic structure forming the CME's geometry a 3-D electron density distribution function 
is defined and placed on the boundary of the magnetic flux rope. This distribution reflects the white-light appearance in the coronagraph observations. With a ray-tracing code which allows to calculate the Thomson scattering by electrons along the observer's line of sight, synthetic coronagraph images can be computed and compared to the white-light structure from the coronagraph images. For further information the reader is referred to Thernisien et al. (2011) and Mierla et al. (2010).

\subsection{The GCS Model}

The GCS modeling technique (Thernisien et al., 2006) was developed in order to reconstruct and analyse the CME's three dimensional geometry from coronagraph images. This forwardmodeling technique for flux rope like CMEs was applied to investigate the 3-D structure of STEREO/SECCHI/COR2 observed CMEs.

\subsubsection{Geometry of the GCS Model}

The GCS model is based on the following four constraints:

- the "legs" have a conical shape

- the curved front is circular

- a circular cross-section of the front part

- a self-similar expansion of the flux rope

All equations of the GCS model can be derived from these constraints. As shown in Figure 38, the GCS geometry consists of two funnel shaped legs with the length $h$. The length of $h$, along the axis through the cone (dashed dotted line), is defined by the center of the Sun, labelled "O", and by the upper end of the cone. The angle between both axes is $2 \alpha$ or $\alpha$ for the half angle, one of the six parameters which define the geometry of the model. Setting the half angle to zero results in a spherical front of the GCS geometry. In this case the GCS model turns into the ice-cream cone model (Fisher and Munro, 1984). The upper part of the model, connecting both legs, is shaped like a tube. The right image in Figure 38 shows an edge-on view of the model consisting of a circle with the varying radius $a$ for the cross-section of the tube-like part and below the tube section the mentioned cone of the legs. Here, $a$ is given by the formula:

$$
a(r)=\kappa \cdot r
$$

with $r$ the distance between solar center and the center of the circle. For this reason $\kappa$ is a constant of the analysed event and can be considered as an aspect ratio of the two orthogonal CME sizes $a$ and $r$. With these parameters we can introduce $h_{\text {front }}$, the distance or height between the center of the Sun "O" and the leading edge of the CME:

$$
h_{\text {front }}=h \frac{1}{1-\kappa} \frac{1+\sin \alpha}{\cos \alpha}
$$

It should be noted that the GCS model does not describe the core material of a three-part structured CME as discussed in Section 2.3. To describe the position and orientation of the flux rope in 3-D space we define the parameters $\phi, \theta$ and $\gamma$ for the Carrington longitude and heliographic latitude of the apex projection on the solar surface and the tilt angle $\gamma$. The intersections of the leg's axes (dashed dotted line in Figure 39) with the solar surface define the foot points of the flux rope and constitute the foot point line (bold line in Figure 39). The tilt angle describes the angle between this line and the solar equator. In this Figure the GCS model is oriented normal to the solar surface and located with the projection of the apex on the solar surface at the given $(\phi, \theta)$ -coordinates where we find the center of the flux rope's foot point line. The legs of the model are located at the opposite ends of the foot point line. For $\gamma=0^{\circ}$ this line is parallel oriented to the solar equator and for $\gamma= \pm 90^{\circ}$ perpendicular. In 2011 Thernisien published a paper containing a detailed mathematical description of the GCS model. 

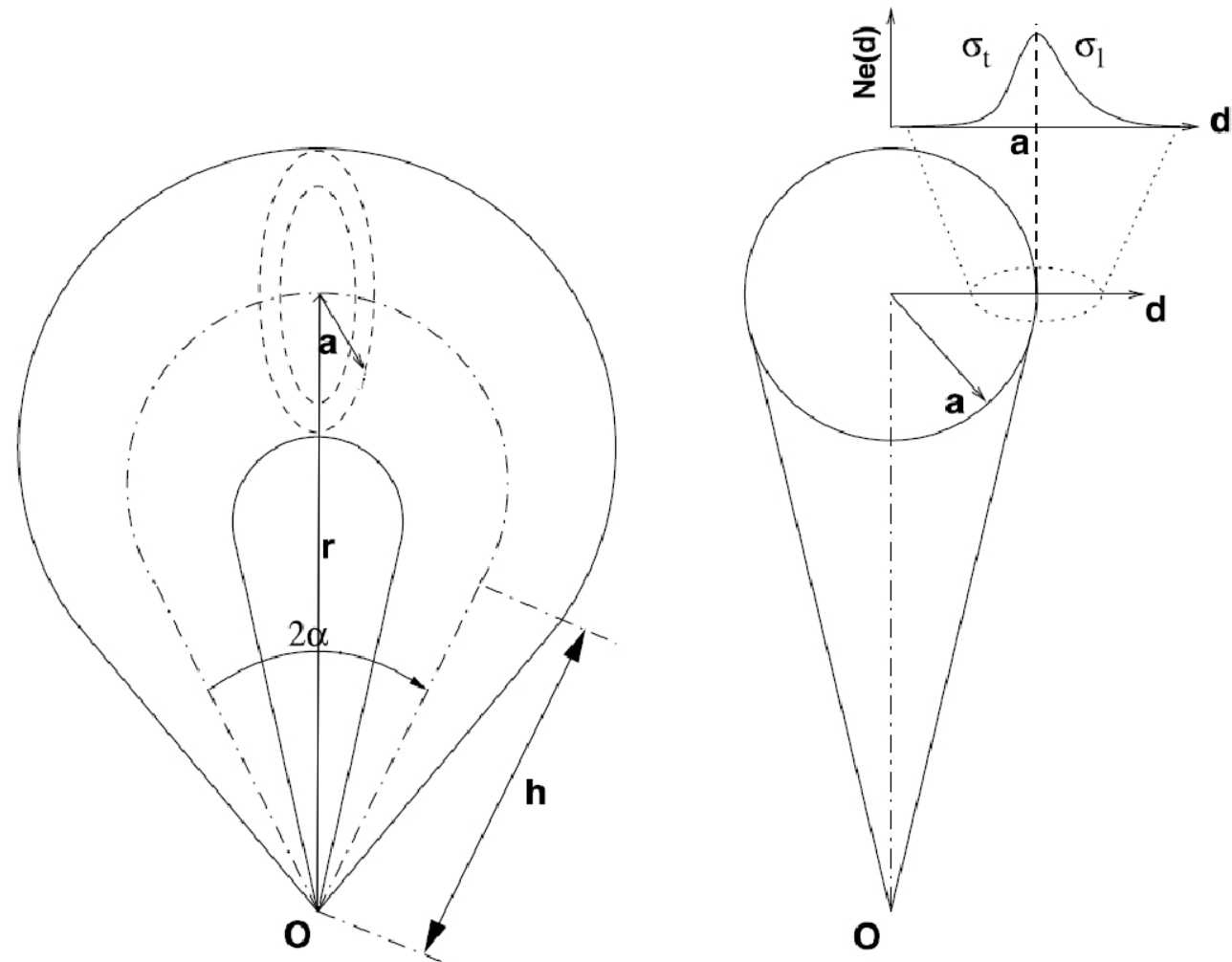

Fig. 38: Face-on (left) and edge-on (right) view of the Graduated Cylindrical Shell (GCS) model. In the upper right is shown the assumed electron density distribution $\mathrm{N}_{e}(d)$ (see Eq. 3). From Thernisien et al. (2006).

\subsubsection{Electron Density Distribution}

Thernisien et al. made an assumption for an electron density distribution which is described by an asymmetric Gaussian like function $\mathrm{N}_{e}(d)$ (see upper right of Figure 38 and Equation 3) alongside the mentioned radius $a$.

$$
N_{e}(d)=N_{e} \exp \left[-\left(\frac{d-a}{\sigma_{s}}\right)^{2}\right] \quad \text { with } \quad \sigma_{s}= \begin{cases}\sigma_{t}, & \text { if } d<a \\ \sigma_{l}, & \text { if } d \geq a\end{cases}
$$

The asymmetric Gaussian profile has its maximum at $a$ on the outer surface of the shell. The density function decreases with a different Gaussian width to the left (trailing segment in direction of propagation) and to the right (leading segment). This is shown at the upper right in Figure 38 where $d$ denotes the distance from the center of the shell along the radius $a$. $\sigma_{t}$ (trailing) and $\sigma_{l}$ (leading) are the widths of the Gaussian function inside and outside the shell, respectively. The electron density is segmented in three dimensional density cubes and each voxel ${ }^{13}$ in a cube comprises the electron density value assumed as given at this position in the 3-D space. This $3-\mathrm{D}$ electron density distribution is required for the calculation of the full Thomson scattering ray-tracing processes to get synthetic coronagraph images of the constructed GCS CME structure. With the GCS morphology and the peak of the electron density distribution placed on the surface of the shell the GCS model is reminiscent of a "hollow croissant". The GCS model does not consider the internal structure of a CME, i.e. it does not represent prominence material as shown in Figure 40. An overview of the GCS parameters is given in Table 7.

\footnotetext{
${ }^{13}$ Voxel: A Voxel names a data point in a 3-D raster graphic and is a composition from the words "volumetric" and "pixel".
} 


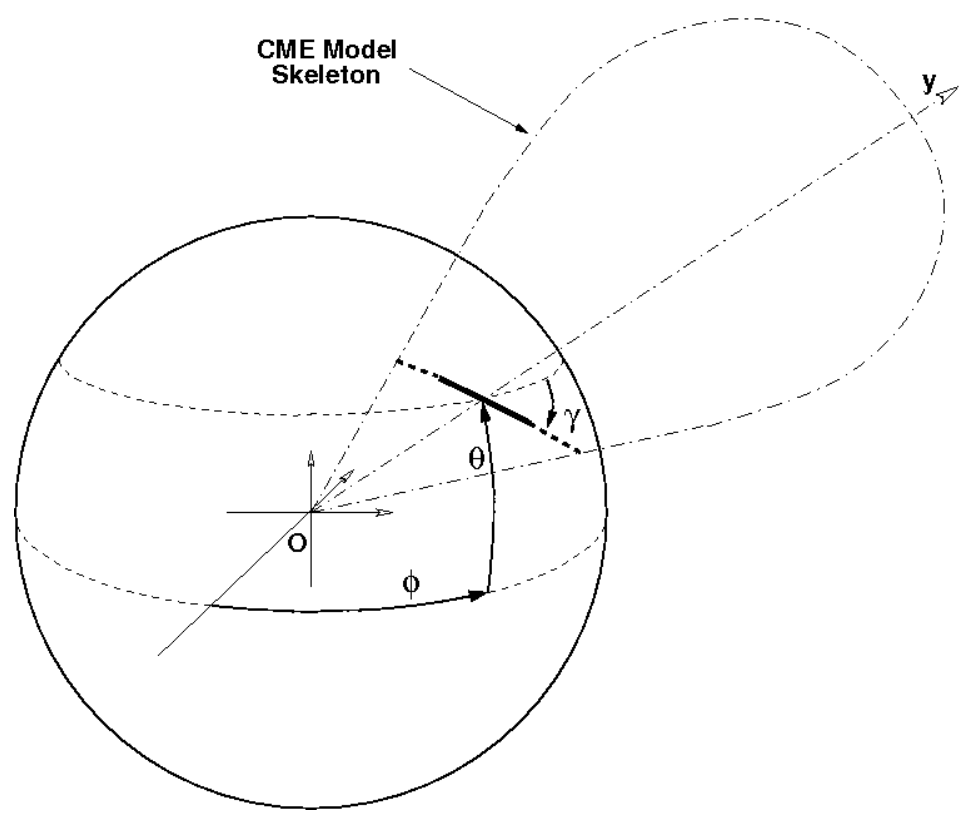

Fig. 39: Position and orientation of the GCS model in 3-D space with the parameters $\phi, \theta$ and $\gamma$ for the Carrington longitude and heliographic latitude of the apex projected on the solar surface and the tilt angle, respectively. From Thernisien et al. (2009).

\begin{tabular}{|c|c|c|}
\hline \multicolumn{2}{|c|}{ Parameter } & Description \\
\hline $2 \alpha$ & [deg] & Angle between the axis of both legs \\
\hline$h$ & {$\left[\mathrm{r}_{\odot}\right]$} & Height of the legs \\
\hline$h_{\text {front }}$ & {$\left[\mathrm{r}_{\odot}\right]$} & Distance from center of the Sun to leading edge (Eq. 2) \\
\hline$\kappa$ & & Aspect ratio of $a$ and $r$ (Eq. 1) \\
\hline$\phi$ & [deg] & $\begin{array}{l}\text { Carrington longitude of the projection of apex } \\
\text { on the solar surface }\end{array}$ \\
\hline$\theta$ & [deg] & $\begin{array}{l}\text { Heliographic latitude of the projection of apex } \\
\text { on the solar surface }\end{array}$ \\
\hline$\gamma$ & [deg] & $\begin{array}{l}\text { Tilt angle of the foot point line relative to } \\
\text { the solar equator }\end{array}$ \\
\hline$a$ & & Cross section radius of the tube section \\
\hline$r$ & & $\begin{array}{l}\text { Distance from center of the Sun to the center } \\
\text { of the cross section }\end{array}$ \\
\hline$N_{e}$ & {$\left[1 / \mathrm{cm}^{3}\right]$} & Electron density factor (Eq. 3) \\
\hline$\sigma_{t}$ & & $\begin{array}{l}\text { Gaussian width of the electron density profile } \\
\text { inside GCS (Eq. 3) }\end{array}$ \\
\hline$\sigma_{l}$ & & $\begin{array}{l}\text { Gaussian width of the electron density profile } \\
\text { outside GCS (Eq. 3) }\end{array}$ \\
\hline
\end{tabular}

Tab. 7: Parameters of the GCS Model and the electron density distribution. From Thernisien et al. (2006).

\subsubsection{Ray-Tracing Code}

For the generation of synthetic coronagraph images with a ray-tracing code we trace the propagating photons along their way from Sun to the electrons of a CME and from there to the observer where the CCD camera of a coronagraph is located. The photospheric light is scattered by the free electrons of the CME along the line of sight to the observer. In general the scattered light can be 
polarised or unpolarised depending on where the observer is located. With the assumed electron density, denoted in electrons $/ \mathrm{cm}^{3}$, the total brightness $B$ can be computed as integration along the line of sight $(\mathrm{LoS})$ and the occurring scattering events.

$$
B=C \int_{\operatorname{LoS}} N_{e}(x, y, z) f(x, y, z) d l
$$

In Equation $4 C$ denotes a constant and the function $f(x, y, z)$ represents the geometrical model. The ray-tracing code provides an implementation of this equation to calculate the total brightness for each pixel of the synthetic coronagraph image. Figure 40 shows exemplarily a pair of synthetic coronagraph images of a GCS fitted CME. The software of the ray-tracing code is available
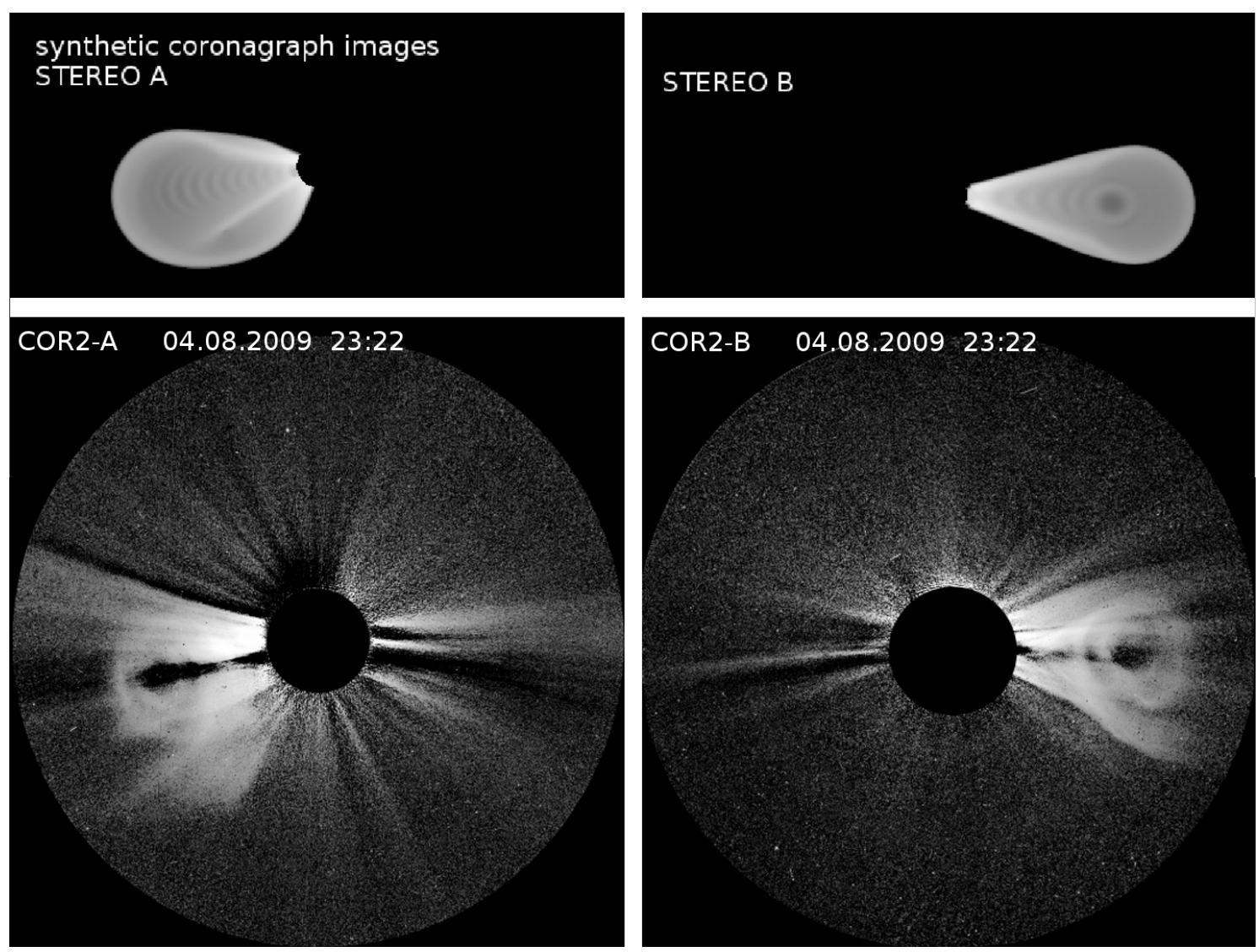

Fig. 40: Top: GCS synthetic coronagraph images for the CME observed on August 4th, 2009 at 23:22 UT with STEREO/SECCHI/COR2 (bottom). The curved interior patterns in both synthetic images are a result of the Moiré effect occurring as a side product of the image processing and therefore describe no physical phenomenon.

within the SolarSoftware ${ }^{14}$ SECCHI package for IDL.

\subsection{STEREO/SECCHI Data Acquisition and Data Processing}

After the presentation of the GCS model the following section is dedicated to the scientific data beginning with the image capture, the image processing on-board the spacecraft, the downlink and further image processing at several facilities to the point of provision of the beacon and scientific STEREO/SECCHI data by the STEREO Science Center (SCC).

${ }^{14}$ SolarSoftware: http://www. Imsal.com/solarsoft/ 


\subsubsection{STEREO/SECCHI Data Provision}

Starting with the recording of a COR2 coronagraph image the image data are send to the onboard computer for image processing. The image processing tasks, which include functions like image compression, event detection and reporting, cosmic ray scrubbing and occulter and region of interest masks, produce an image file prepared for downlink to Earth (Howard et al., 2008). The STEREO/SECCHI/COR2 science data consist of total brightness $2048 \times 2048$ pixel images and polarised component images. The processed data are written to the solid state recorder. There are data processing level which describe the status of processing. On the spacecraft the SECCHI data are at Level 0 (see Table 8). During radio contact to the Deep Space Network (DSN) data can be transferred with a telemetry rate of maximum $720 \mathrm{kbps}$ depending on the distance to the spacecraft. The Payload Operation Center (POC) for SECCHI located at the Naval Research Laboratory (NRL) receives the telemetry data via the Mission Operations Center (MOC) operating at the John Hopkins University's Applied Physics Laboratory (APL). After further steps of data processing the science data are available at the NRL at Level 0.5, i.e. they are stored as FITS files containing uncompressed 16-bit images with a header consisting of telemetry and auxiliary information.

\begin{tabular}{|c|c|c|}
\hline Level & Source & Description \\
\hline Packets & Spacecraft $\rightarrow$ MOC & $\begin{array}{l}\text { Packets transmitted by the spacecraft } \\
\text { to the DSN }\end{array}$ \\
\hline Level-0 & MOC & $\begin{array}{l}\text { Sorting of packet files and removing } \\
\text { of duplicates }\end{array}$ \\
\hline Level-0.5 & $\mathrm{POC} / \mathrm{DPF}^{*}$ & $\begin{array}{l}\text { FITS files: uncompressed } 16 \text {-bit images } \\
\text { Header with telemetry and auxiliary data }\end{array}$ \\
\hline Level-1 & $\begin{array}{l}\text { User Workstation } \\
\text { (Solarsoft) }\end{array}$ & $\begin{array}{l}\text { "on the fly" calibrated FITS as result } \\
\text { of FITS files and IDL procedures }\end{array}$ \\
\hline Level-2 & $\begin{array}{l}\text { User Workstation } \\
\text { (Solarsoft or DPF) }\end{array}$ & $\begin{array}{l}\text { Data products as a result of several FITS } \\
\text { files, e.g. movies, Carrington maps, } \\
\text { polarised brightness images }\end{array}$ \\
\hline Level-3 & $\begin{array}{l}\text { User Workstation } \\
\text { (Solarsoft) }\end{array}$ & $\begin{array}{l}\text { Derived quantities like electron density or } \\
\text { temperature ratios }\end{array}$ \\
\hline
\end{tabular}

Tab. 8: Data processing level definition for STEREO/SECCHI data, adapted from Howard et al., 2004. (*) DPF: Data Processing Facility.

Additional to the normal science data telemetry downlink during radio contact to the Deep Space Network (DSN) the instruments on-board STEREO produce a low-rate data stream which provides the space weather beacon data. In time periods without DSN radio contact the continuous space weather beacon stream is received by several antenna partner stations ${ }^{15}$ around the world who collect the beacon data and send it to the STEREO Science Center (SSC) via internet. There the telemetry data are processed and finally provided as data files in the FITS format to the public. After reception of the telemetry data by the antenna partners within a few minutes ( $\sim 10 \mathrm{~min})$ the beacon data are processed and provided online via the $\mathrm{SSC}^{16}$. With a delay of about 3 hours since the original recording the beacon data are available for space weather forecast analysis. The NRL provides via a web interface access to the data of all five SECCHI instruments with the "SECCHI Flight Images Query Form"17. The data used for CME modeling are taken from this site. Further detailed information about the data processing can be found in Howard et al., 2008 and in the technical documentations by Thompson, 2004 and Howard et al., 2004.

\footnotetext{
${ }^{15}$ Antenna partner stations: Bochum (GER), Kiel (GER), Toulouse (FRA), Chilbolton (GBR), Koganei (JPN), Jeju (KOR), Laurel, MD (USA).

${ }_{16}^{16}$ Space weather beacon data: http://stereo-ssc.nascom.nasa.gov/data/beacon/

${ }^{17}$ SECCHI Flight Images Query Form: http://sharpp.nrl.navy.mil/cgi-bin/swdbi/secchi_flight/img_ short/form
} 


\subsubsection{Software Requirements and Installation}

Additional to the FITS files of the observed CME the user needs the 3-D GCS CME modeling tool which is part of SolarSoftWare ${ }^{18}$ (SSW). SolarSoft is a system of software libraries, databases and system utilities developed by the Solar \& Astrophysics Laboratory at Lockheed Martin (http://www.Imsal.com). The software suite was developed for several solar missions like Yohkoh, SOHO, SDAC and STEREO which are integrated as software packages within SolarSoft. The Interactive Data Language (IDL) needs to be installed on a Linux or Mac operating system for the usage of Solarsoft. For installing SolarSoft it is important to choose the packages "STEREO" and "SECCHI". And for a correct data processing the SolarSoft Database packages "SECCHI Background cal (Standard) stereo/secchi/backgrounds/a/monthly_min" are necessary, and the corresponding for spacecraft STEREO-B. The setup for starting IDL with the SSW environment is described at ww. lmsal.com/solarsoft/ssw_setup.html.

\subsubsection{Data Usage for Modeling}

This section illustrates the data preparation for forward-modeling of a CME with STEREO/SECCHI /COR2 data. As an example the CME detected on June 4th, 2010 was chosen when the two STEREO spacecraft were separated by $143^{\circ}$ in heliographic longitude. The CME was identified in the synoptic movies of the COR2 coronagraphs available at http://secchi.nrl.navy.mil/ sccmovies/. Figure 41 shows two images of the CME observed with the coronagraphs COR2-A and -B. The coronagraph's occulter is placed in front of the solar disk to block the direct intense Sun light. The position of the solar disk is denoted with a white circle. It should be noted that

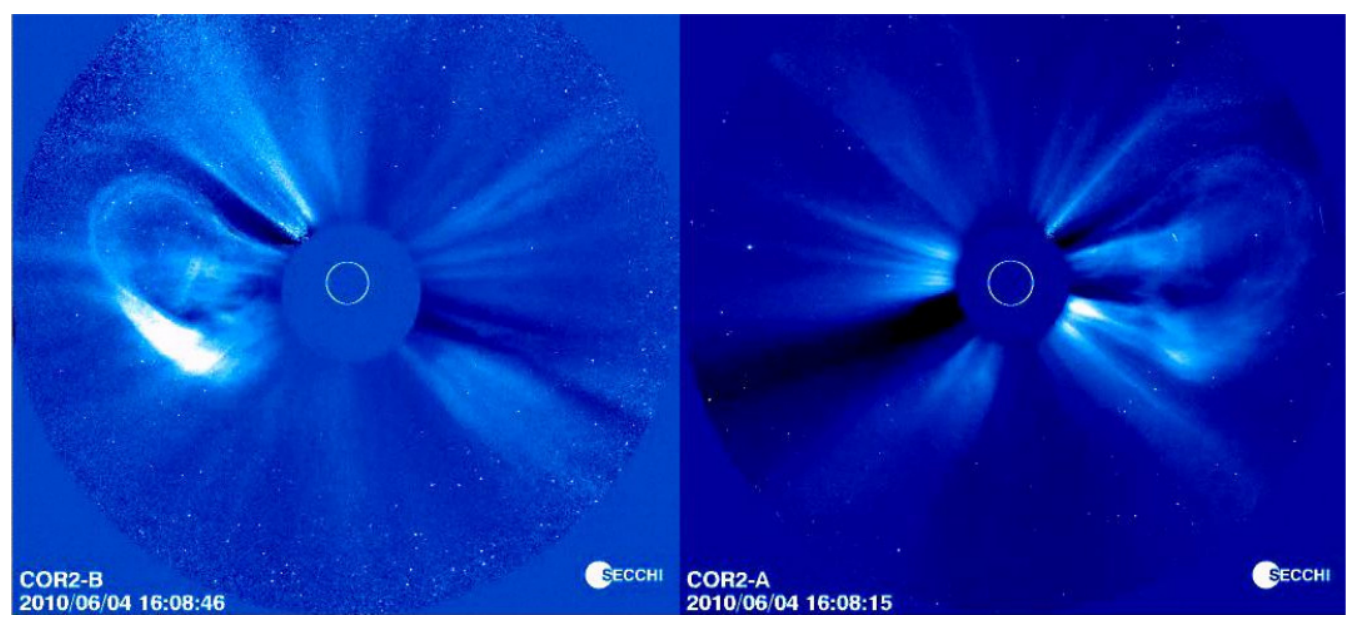

Fig. 41: An image pair of the CME detected on June 4th, 2010 at 16:08 UT with STEREO/SECCHI/COR2-A and -B from http://secchi.nrl.navy.mil/sccmovies/. At this point of time the CME is largely expanded and best visible in the field of view of COR2 and therefore best suited for modeling. The white circle in the center of the blue pigmented occulter denotes the size of the solar disk.

there is an off-pointing in the COR2-B images concerning the white circle for the solar disk. The CME was observed at different position angles (PA) of $90^{\circ}$ and $270^{\circ}$, respectively as shown in Figure 41.

The following description of the GCS modeling procedure is based on the tutorial provided by $\mathrm{NRL}^{19}$. In order to fit the CME with difference images COR2-A and -B FITS files are required

\footnotetext{
${ }^{18}$ SolarSoftware: www.lmsal.com/solarsoft/ssw_install_howto.html, SolarSoft Database: www.lmsal.com/ solarsoft/sswdb_install.html

${ }^{19}$ GCS modeling tutorial: http://secchi.nrl.navy.mil/synomaps/scraytrace/dobo/examples.html\# tutrtsccguicloud
} 
for a time $t_{2}$ when the CME is best visible (here: at 16:08 UT on June 4th, 2010) and for the time $t_{1}$ before the CME enters the field of view (here: 11:08 UT on June 4th, 2010). The STEREO/SECCHI/COR2 synoptic movies allow to find quickly the time-stamps. Via the mentioned website for the SECCHI FITS files (Section 5.3.1) the user gets for each point in time and coronagraph three FITS files, thus in sum 12. It is important that the COR2 files are chosen as triples captured in sequence by SECCHI. This is necessary because during the subsequent course total brightness images will be calculated from each of these three polarised brightness images. In addition one STEREO/SECCHI/EUVI-A and -B FITS file for the time $t_{2}$ is processed. The wavelength of the EUVI images can be chosen depending on the source region of the CME. After downloading these files all of them are processed with several IDL routines which can be found in the Appendix A.1. Among other IDL routines the FITS files are processed with the secchi_prep.pro routine $^{20}$. This routine allows the calibration and image correction of raw image files with level 0.5 into level 1.0 or 2.0 for further analysis. With the preparation of all images a background subtraction is applied based on the SECCHI background images stored in the Solarsoft Database. At the end a difference image is generated from both COR2-A images (time-stamp $t_{1}$ and $t_{2}$ ) in order to substract the scattered light from the F corona. In this step the difference image is also rescaled and processed for better visualisation. The same is done for the COR2-B images and also for both EUVI images, except the difference imaging. In the final step the headers and images are delivered to the GCS routine rtsccguicloud.pro at procedure call.

\subsection{CME Modeling with the GCS Technique}

The GCS modeling is demonstrated for six CME events in the following sections. The individual CME characteristics are discussed in addition to fitting difficulties. The last section describes how a CME's source region is identified and localised for further analysis.

\subsubsection{CME on June 4th, 2010}

After processing of the image data and the subsequent program start of the GCS tool its graphical user interface is presented to the user (Figure 42). The slider for the six GCS parameters (longitude, latitude, tilt angle, height, aspect ratio, half angle) are shown in the control panel on the left. The parameters can be adjusted to fit the GCS geometry to the CME white-light structure which is presented in the right images (top: COR2-A, bottom: COR2-B). The EUVI images present the solar disk at $195 \AA$ (top: EUVI-A, bottom: EUVI-B) in the center frame.

The green wireframe overlay in the right images represents the modifiable GCS geometry and in this way all points on the surface of the "hollow croissant". As mentioned, the assumed electron density distribution in the model has its maximum on the GCS surface in other words on the green wireframe. In the coronagraph image, the observed brightness displayed in a pixel corresponds to the integrated electron density distribution along the line of sight. Thus the observable features with a peak in brightness are fitted as CME front. To determine an appropriate GCS-like CME morphology we compare the GCS geometry to the CME white-light structure of the observation. The real-time computation of the wireframe takes into account the position, orientation and field of view (FOV) of both spacecraft and calculates the wireframe synchronously as an overlay to both coronagraph images. Hence the fit can be done interactively until the wireframe projection matches to the CME white-light structure in both views. The individual steps on how to best adjust the six parameters for an appropriate fit are described by Thernisien et al. (2009).

The foot points of the legs of the flux rope are labelled with green plus signs projected on the solar surface in the EUVI images (Figure 43). The apex of the CME's leading edge is projected on the solar surface and labelled with a green asterisk. Foot points and apex of a flux rope located on the backside of the Sun are white coloured. If the CME's source region is visible in the EUVI

\footnotetext{
${ }^{20}$ Colaninno, R.: 2006-2010, The SECCHI_PREP Homepage, Naval Research Laboratory (NRL/GMU), http: //secchi.nrl.navy.mil/wiki/pmwiki.php?n=Main.HomePage.
} 


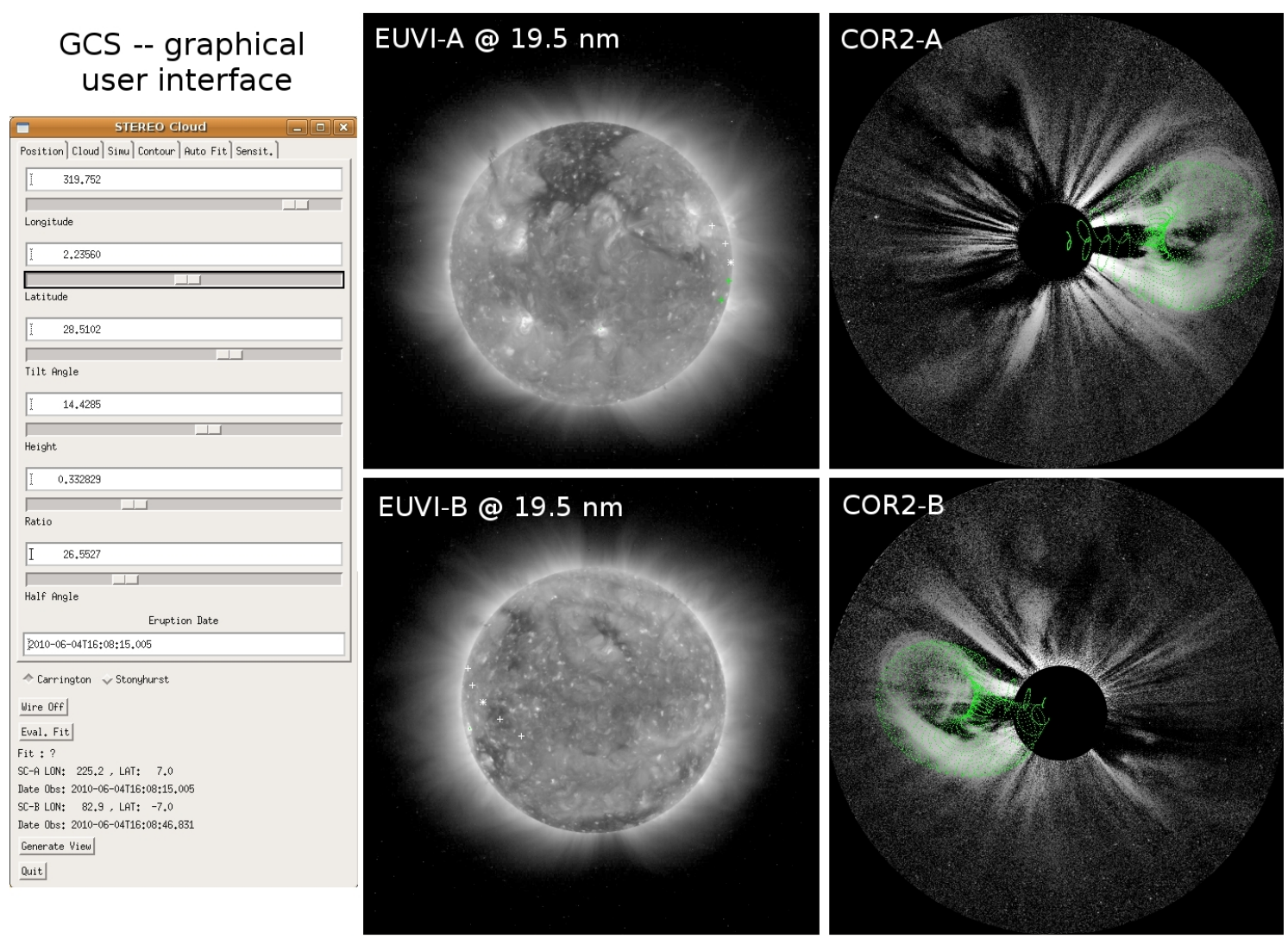

Fig. 42: Graphical user interface of the GCS modeling program rtsccguicloud.pro used with COR2 data of the CME detected on June 4th, 2010 at 16:08 UT. Top left: EUVI-A image at $195 \AA$, top right: COR2-A image with a green wireframe representing the GCS fit, bottom left: EUVI-B at $195 \AA$, bottom right: COR2-B image.

images it can be directly compared to the CME's apex and foot point locations.

The relationship between CMEs and their associated source regions is based on the results and the introduced CME scheme from Cremades and Bothmer (2004), see Section 2.3.4. They analysed in their study the relations between the CME morphology visible in SOHO/LASCO coronagraph images and the properties of their associated source regions on the solar surface. Cremades and Bothmer suggested a cylindrical geometry for the CME's topology. They found from their CME and source region correlations that for this cylindrical geometry the longitudinal symmetry axis depends on the orientation, position and length of the source regions's neutral line visible on the solar disk.

This implies that the position, extension and orientation of the source region provides clues to the position (longitude, latitude), orientation (tilt angle) and elongation (half angle) for modeling the CME as GCS flux rope. In this context the length of the foot point line corresponds to the extent of the angle $\alpha$ between the axes of both legs. The results from the GCS modeling shown in Figure 42 which describe the white-light appearance of the CME observation in the best way of the author's knowledge are listed in Table 9 at the end of this section.

\subsubsection{CME on November 16th, 2007}

CMEs observed in 2007 during the first months of the STEREO mission exhibited a very similar white-light appearance in the COR2-A \& -B FOV due to the low separation angle between both spacecraft. This changes with increasing separation angle as demonstrated with the CME shown 

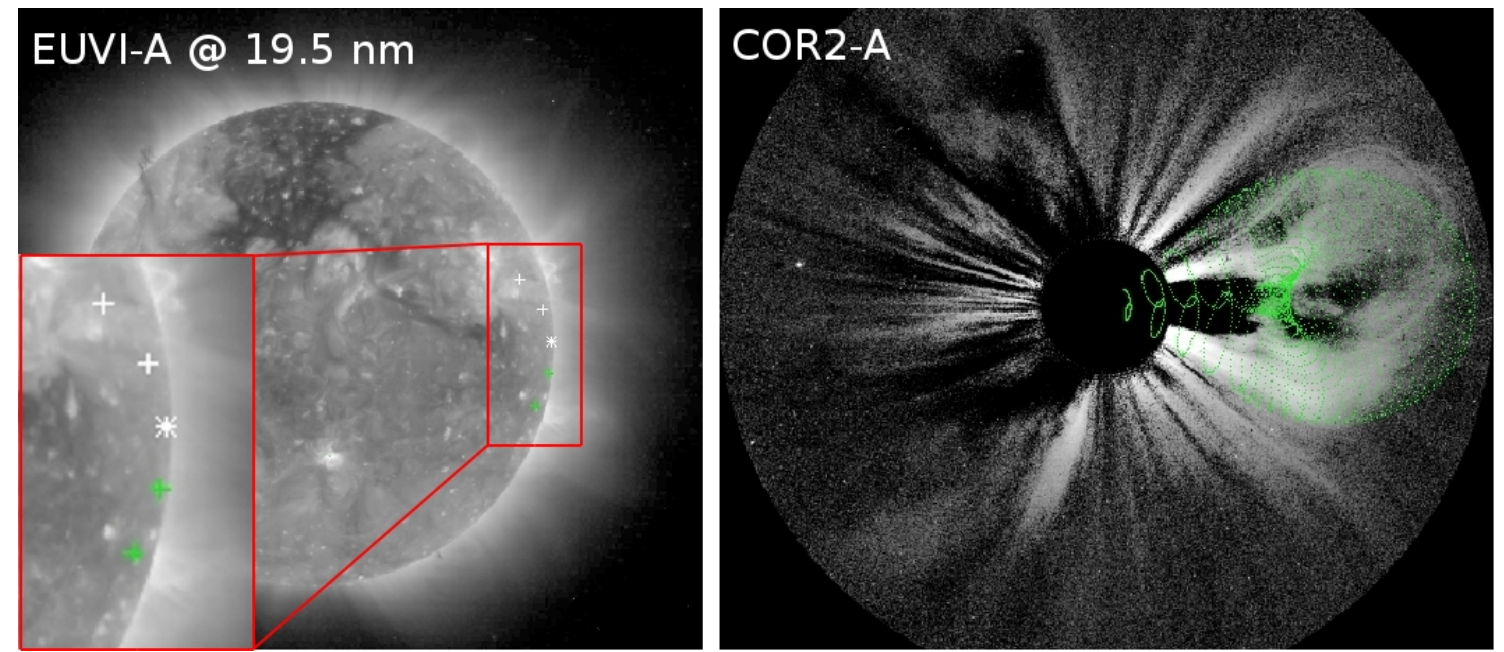

Fig. 43: Left: foot points of the legs of the flux rope for the CME observed on June 4th, 2010 at 16:08 UT (right). The inner and outer foot points of the legs are labelled with green and white plus signs and the apex of the GCS leading edge with an asterisk. The green (front side) and white (back side of Sun) plus signs indicate the $\mathrm{CME}$ as limb event.

in Figure 44 which was observed on November 16th, 2007 when both spacecraft were separated by $40^{\circ}$. The CME appears in both coronagraph's FOV with the same position angle (PA) but with a different white-light structure. The CME's direction of propagation calculated from GCS modeling is indicated by the red arrow in the centered image. For STEREO-A (located at $220^{\circ}$ Carrington longitude) the CME (at $323^{\circ}$ ) is seen just behind the west limb whereas from STEREO-B (located at $180^{\circ}$ ) the $\mathrm{CME}$ is propagating backwards to the right. The results from GCS modeling are listed in Table 9 at the end of this section.

\subsubsection{CME on November 3rd, 2008}

At the end of the second mission year both spacecraft were separated by $82^{\circ}$ in November 2008 . At this time and later when both spacecraft are separated by about $90^{\circ}$ a $\mathrm{CME}$ seen from one spacecraft as limb event is visible from the other as front or backside Halo CME. This reveals very different views to a CME and very good conditions for the CME's 3-D resolution. In contrast to the early mission phase CMEs occur more often at different position angles with increasing separation angle between both spacecraft. This is presented in Figure 45 for the CME observed on November 3rd, 2008. From the COR2 observations it is obvious that the CME is propagating from the front side of the Sun between STEREO-A (located at $262^{\circ}$ Carrington longitude) and -B (at $\left.181^{\circ}\right)$. The GCS modeling reveals a CME propagation $\left(229^{\circ}\right)$ directed to Earth which is located at $221^{\circ}$ Carrington longitude. Hence the CME is seen from SOHO as front side Halo CME which might have a possible geo-effective impact at Earth. This CME event is a good example for applying the GCS modeling technique in order to judge to which extent the CME is moving towards Earth. The GCS modeling results are provided in Table 9. An inspection of the solar wind data measured by ACE revealed no signatures of a CME. Due to the lack of indications in the solar wind parameters (magnitude of magnetic field, $B_{z}$ component of magnetic field, proton velocity, number density and proton temperature) it is assumed that the CME did not hit the ACE spacecraft and neither Earth. The CME direction in heliospheric latitude of $+15^{\circ}$ suggests that the CME may have propagated northwards out of the ecliptic and over the Earth's orbit. The bright point visible in the COR2-B images (top right) shows the planet Mercury. 


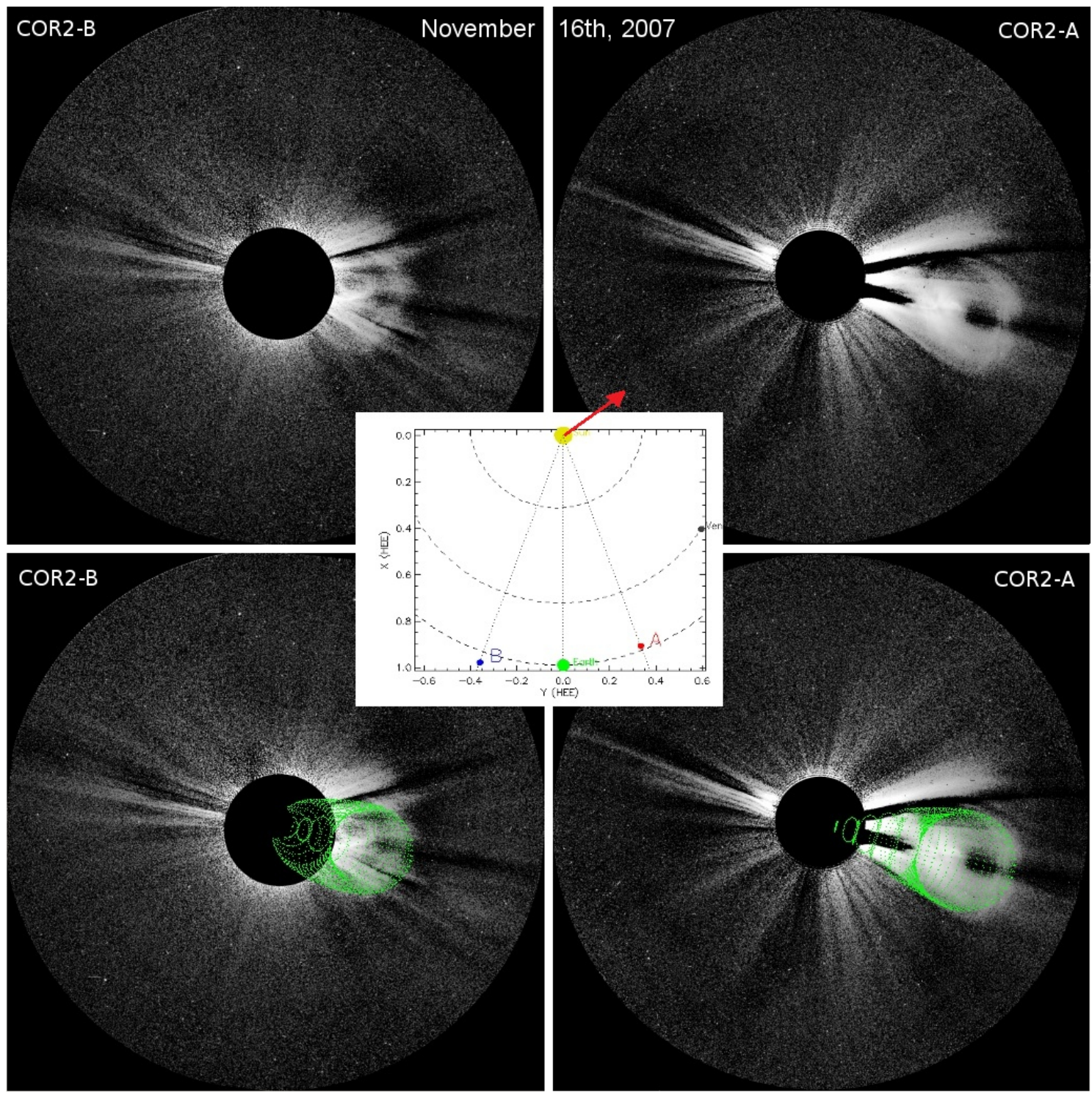

Fig. 44: A CME observed on November 16th, 2007 at 15:22 UT. The CME's direction was calculated from GCS modeling to $323^{\circ}$ Carrington longitude (red arrow, center frame). The STEREO-A and -B spacecraft (center frame) were located at this time at $220^{\circ}$ and $180^{\circ}$ Carrington longitude, respectively.

\subsubsection{CME on February 2nd, 2011}

In February 2011 both STEREO spacecraft reached a separation angle of $180^{\circ}$ which allowed at that time the first $360^{\circ}$ view of the Sun. For CMEs which appear as front side Halo CME for one spacecraft and as back side halo CME for the other observatory additional data are needed like its source region location or coronagraph observations from SOHO to determine the CME's direction of propagation unambiguously. A CME observed at this time on February 2nd with a direction of $0^{\circ}$ in Carrington longitude is shown in Figure 46. This CME seen from STEREO-A at $274^{\circ}$ and STEREO-B at $94^{\circ}$ Carrington longitude appears for both spacecraft as limb CME. It is propagating towards the back side of the Sun as seen from Earth (at $187^{\circ}$ longitude). The leading edge of this CME exhibits a large distortion. Such CMEs poses a problem for fitting with the GCS modeling technique. The GCS model is based on the assumption of a circular curved CME front and the modeling technique does not allow to modify the curved front of the model. 


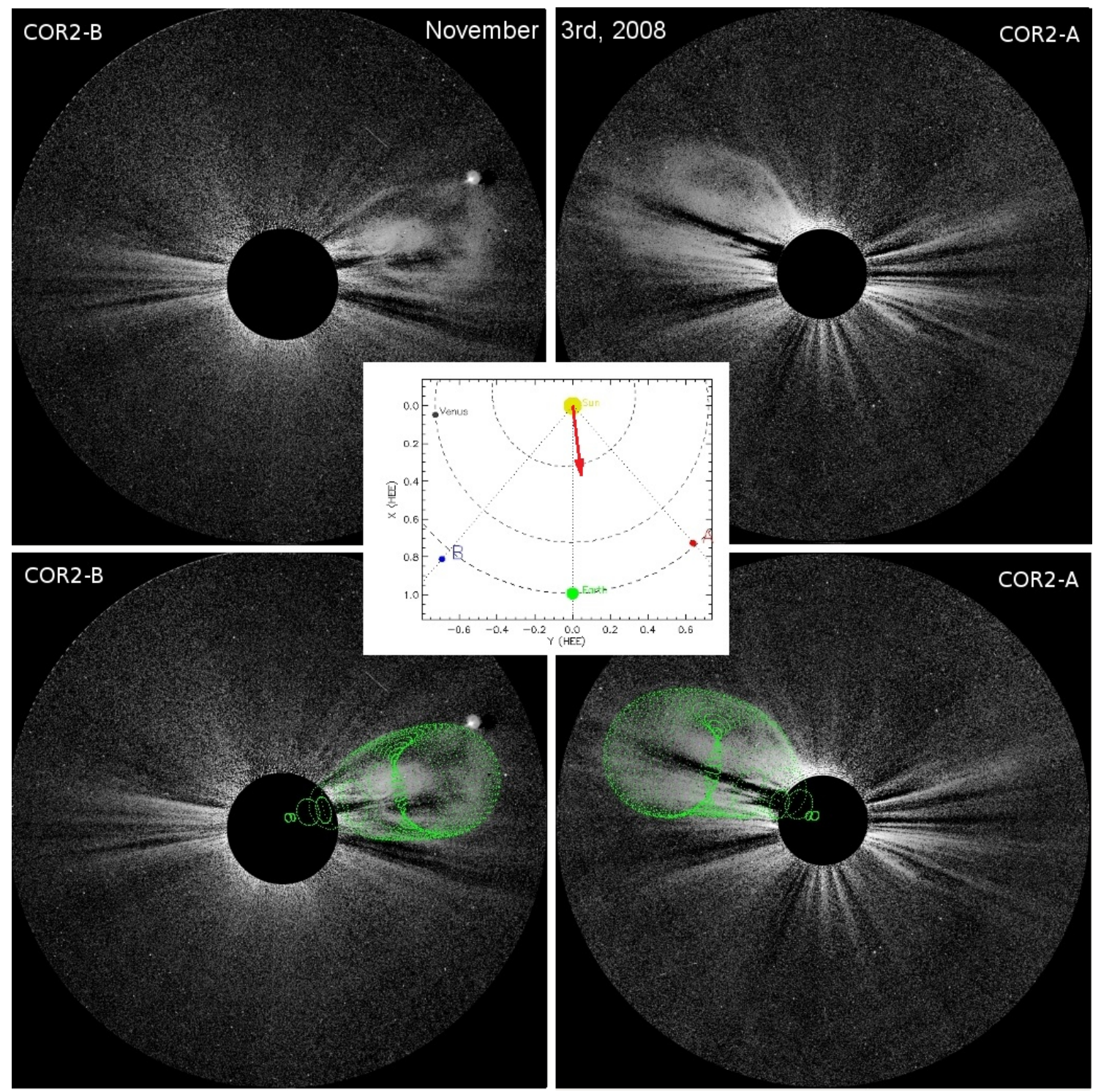

Fig. 45: A CME observed on November 3rd, 2008 (07:22 UT) at different position angles in the COR2 images. The CME seen from STEREO-A (located at $262^{\circ}$ Carrington longitude) and STEREO-B (at $181^{\circ}$ ) is directed to Earth (located at $221^{\circ}$ ) with $229^{\circ}$ in Carrington longitude (red arrow).

Fitting a distorted leading edge affects the determination of the CME's GCS height parameter. A distorted leading edge could be caused by the magnetic topology of the CME's associated source region (SR). A source region possess a neutral line which separates the bipolar magnetic regions. A source region with a kink in the neutral line might generate such a CME. Furthermore the source region's neutral line corresponds with the previous mentioned GCS foot point line which cannot be modified in the GCS model. It is also possible that the curved front of a CME ejected into the solar wind might be deformed by the inhomogeneous conditions (speed, density) of the ambient solar wind (Savani et al., 2010). 


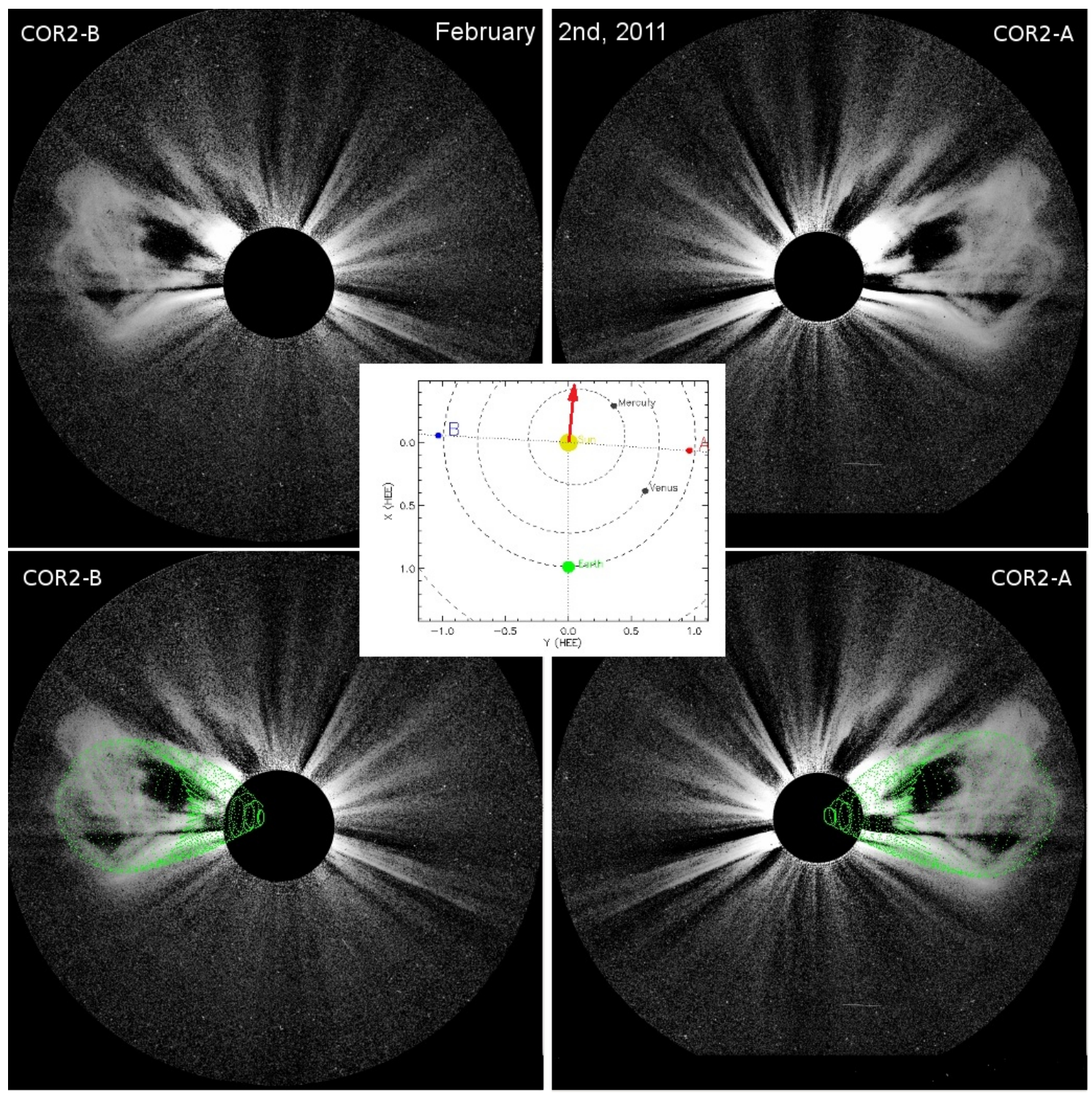

Fig. 46: A limb CME observed on February 2nd, 2011 at 02:08 UT from STEREO-A (located at 274 ${ }^{\circ}$ ) and STEREO-B (at $94^{\circ}$ Carrington longitude). This CME which is directed to $0^{\circ}$ in Carrington longitude (red arrow) was visible as back side Halo CME seen from SOHO/Earth (at $187^{\circ}$ ). Note that the distortion of the CME front cannot be fitted with the GCS model which assumes a curved CME front.

\subsubsection{CME on April 26th, 2008}

In 1985 Sheeley et al. found that interplanetary shock waves often occur together with large interplanetary CMEs which are as a result considered as their source. Such shock waves driven by fast CMEs occur if the CME's speed significantly exceeds the velocity of the ambient solar wind (Aschwanden, 2006 chapter 17.9.3). A shock can be detected in the in situ solar wind data measured for instance by the ACE spacecraft. Indicators for a shock are a sudden increase in proton speed and temperature. In some cases a shock can be also observed in coronagraph images additional to the CME white-light structure (Vourlidas et al., 2003; Vourlidas and Ontiveros, 2009). If so the shock appears in front of the CME's leading edge or also surrounding the CME white-light structure with a diffuse and faint decreasing brightness.

The CME observed on April 26th, 2008 is characterised by a bright leading edge (red dots in the top images of Figure 47) with a shock moving ahead. As shown in the center frame of this Figure 


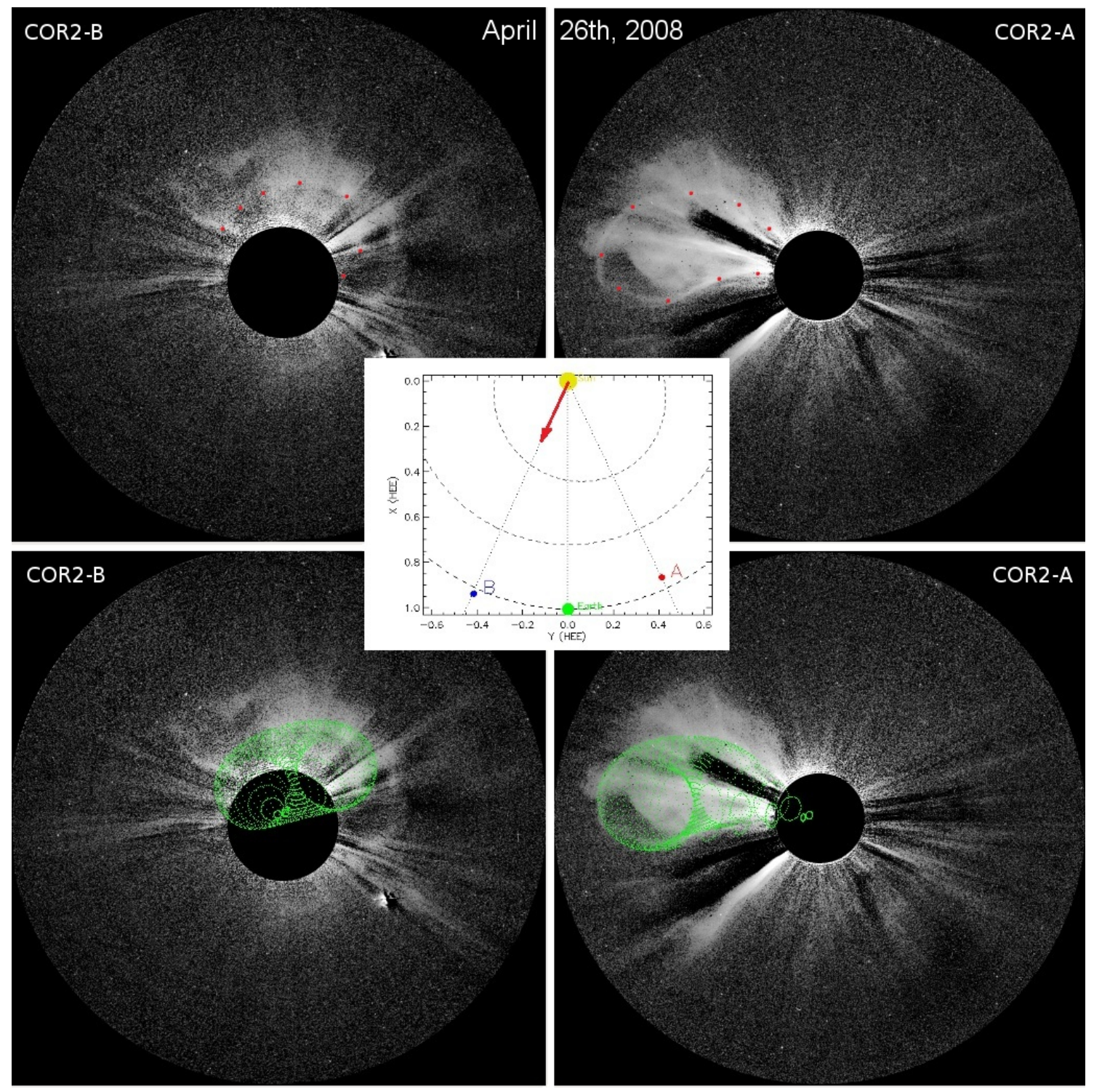

Fig. 47: A CME with a shock in front of its leading edge (red dotted) observed on April 26th, 2008 at 17:52 UT. The CME is directed towards STEREO-B (located at $202^{\circ}$ Carrington longitude) which is separated by $50^{\circ}$ in longitude to STEREO-A. The CME's direction of propagation (red arrow) is calculated from GCS modeling.

(red arrow) the CME is directed towards STEREO-B (located at $202^{\circ}$ Carrington longitude) which is separated by $50^{\circ}$ from STEREO-A. The CME's leading edge (red dotted) can be differentiated to the ambient shock material in the COR2-A image. This material appears diffuse and faint in brightness in front of the bright leading edge which is defined and clear in structure. The whitelight appearance of the shock material makes the CME looking like a partial halo CME in the COR2-B image (top row). The shock material in COR2-B surrounds the CME (which is directed towards the observer) with an angular width up to $\approx 180^{\circ}$ around the line of sight. The appearance of the CME similar to a partial halo CME can lead to wrong fit results if the shock material is included in modeling the shape. This affects in particular the GCS parameters half angle, aspect ratio and height. The GCS fit of the CME is shown in the images at the bottom. In some cases it is difficult to identify correctly a possible shock and to distinguish between this one and the CME. 


\subsubsection{CME on March 12th, 2011}

As described in Section 5.3.1 the STEREO spacecraft provide a data stream with low resolution beacon data for space weather forecast purposes. For a comparison with the high resolution science data the GCS modeling technique was also applied to the COR2 beacon data for a few CME events. The advantage of the beacon data is the early availability, three hours after recording on-board STEREO which is helpful for forecasting space weather conditions. But the low resolution of beacon data limits the possibilities of performing a reliable GCS CME fit when the high resolution science data are not yet available. In Figure 48 the COR2 coronagraph images from the beacon data stream (left) are contrasted with the images from the science data stream (center) for a CME observed on March 12th, 2011. At this time both STEREO spacecraft were separated in longitude
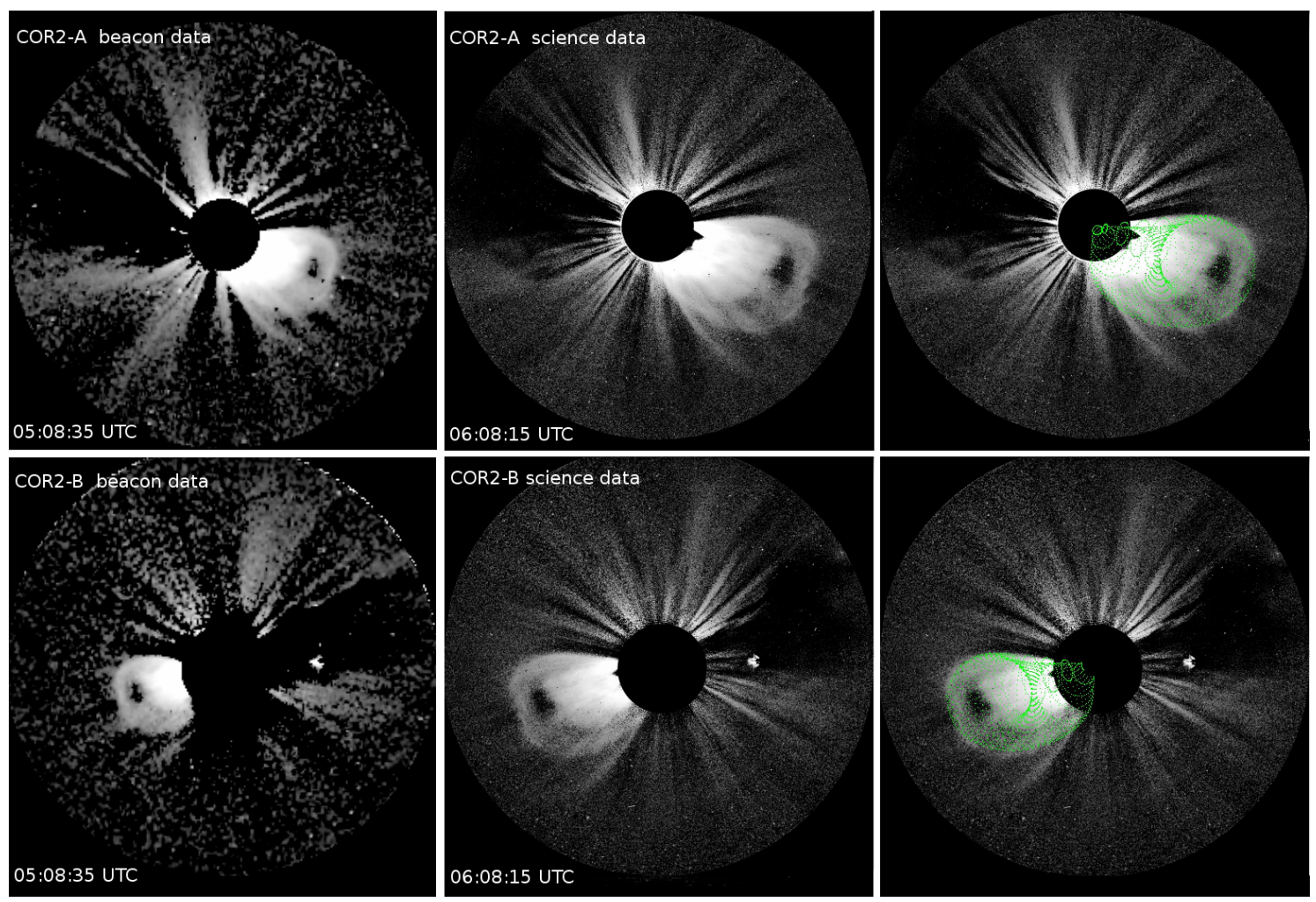

Fig. 48: A CME observed on March 12th, 2011 at 06:08 UT with STEREO/SECCHI/COR2-A (top row) and COR2-B (bottom row). The low resolution coronagraph images from the beacon data stream (taken at 05:08 UT) are shown on the left. Due to a data gap no beacon data are available for the time 06:08 UT corresponding to the displayed science data. The images from the high resolution science data stream are presented in the middle and on the right the same with the GCS CME fit.

by $177^{\circ}$. The CME was directed $43^{\circ}$ to the right of the STEREO-A Sun line. The GCS CME fit shown on the right is based on the COR2 science data which are available on average two days after image recording. In this case a GCS CME fit with beacon data is possible but in most other cases the CME is badly recognisable and not very suitable for fitting. The bright point in the COR2-B images to the right of the Sun constitutes the planet Venus.

\subsubsection{Sources of Error using GCS}

After the modeling of one or more CMEs there arises the question about the possible sources of error. For a compilation of a CME fit one can adjust each of the six GCS parameters in small steps (e.g. longitude in steps of $0.0036^{\circ}$ ) but the step size doesn't allow conclusions to a fitting 


\begin{tabular}{rrrrrrrr}
\hline CME date & \multicolumn{9}{c}{ GCS Parameter } & & & & \\
& $\phi$ & $\theta$ & $\gamma$ & $\mathrm{h}_{\text {front }}$ & $\kappa$ & $\alpha$ & S/C Sep. \\
dd.mm.yyyy & {$[\mathrm{deg}]$} & {$[\mathrm{deg}]$} & {$[\mathrm{deg}]$} & {$\left[r_{\odot}\right]$} & {$[-]$} & {$[\mathrm{deg}]$} & Angle [deg] \\
\hline 16.11 .2007 & 323 & -14 & 5.6 & 11.6 & 0.3 & 18.4 & 40.5 \\
26.04 .2008 & 203 & 6 & 14.5 & 15.1 & 0.2 & 14.0 & 49.5 \\
03.11 .2008 & 229 & 15 & 0.0 & 16.9 & 0.3 & 12.0 & 81.8 \\
04.06 .2010 & 320 & 2 & 28.5 & 14.4 & 0.3 & 26.6 & 142.6 \\
02.02 .2011 & 0 & 4 & 0.0 & 13.5 & 0.4 & 33.5 & 179.4 \\
12.03 .2011 & 177 & -17 & 8.4 & 14.4 & 0.3 & 20.4 & 177.0 \\
\hline
\end{tabular}

Tab. 9: The GCS modeling results for the six presented CMEs. From left to right: Carrington Longitude, Heliospheric Latitude, Tilt Angle, Height, Aspect Ratio and Half Angle. The last column lists the separation angle between both STEREO spacecraft (S/C).

error of this parameter. To estimate the errors of a GCS fit the following sources of error should be considered.

\section{The CME's white-light appearance:}

For the modeller problems may occur when the CME front is not clearly visible because it is "washed-out" in the ambient background (Thernisien et al., 2009). CMEs which exhibit not only one single front and CMEs with a distorted leading edge (see Figure 46 in Section 5.4.4) are also difficult to model because the GCS model describes CMEs with a circular curved front (Thernisien et al., 2011). In some cases it is hard to distinguish between the CME's shock and the CME's leading edge which can change the morphology of the fit to a large extent. An example of such a CME and its fit is shown in Figure 47 in Section 5.4.5.

Information about the source region of a CME can help to improve the GCS fit of a CME. If the source region of a CME is identified and visible in the STEREO/SECCHI/EUVI images then these images which are loaded into the GCS modeling software can be taken into account for modeling the CME.

The relationship between CMEs and their associated source regions is based on the results and the introduced CME scheme from Cremades and Bothmer (2004), see Section 2.3.4. They analysed in their study the relations between the CME morphology visible in SOHO/LASCO coronagraph images and the properties of their associated source regions on the solar surface. Cremades and Bothmer suggested a cylindrical geometry for the CME's topology. They found from their CME and source region correlations that for this cylindrical geometry the longitudinal symmetry axis depends on the orientation, position and length of the source regions's neutral line visible on the solar disk.

The GCS modeling software displays the foot points of the GCS flux rope fit on the STEREO/ SECCHI/EUVI images (Figure 43). These foot points correspond to the longitudinal symmetry axis and therefore can be directly compared to the identified source region in the EUVI images. With this the GCS flux rope position in longitude and latitude and the orientation given by the GCS tilt angle can be adjusted. Therefore source regions visible in EUVI can help to reduce uncertainties in determining the CME's GCS longitude, latitude and tilt angle from COR2 observations. However, as the CME can be deflected and/or rotated during its propagation away from Sun the source region parameters cannot be compared one-to-one with the GCS CME parameters (Thernisien et al., 2006).

\section{"Bandwidth" for adjustment of GCS parameters:}

For a generated GCS fit (as a set of six parameters) each individual parameter exhibits a certain bandwidth for a modification in which bandwidth the parameter and therefore the complete fit 
describes the white-light appearance of the CME observation to a certain goodness. This of course depends on the user's subjective judgement. In this context Thernisien defined a merit function to estimate how well the model is able to reproduce an observed CME's white-light appearance (Thernisien et al., 2009). With this merit function describing the "Goodness of fit" Thernisien performed a sensitivity analysis for a GCS study of $26 \mathrm{CMEs}$. The mean error values of the six GCS parameters were calculated for a $10 \%$ decrease of this merit function. The results of this sensitivity analysis are summarised in Table 10 and can serve as an estimation for a systematic error based on the specific modeling technique. These results reveal that the deviations of the

\begin{tabular}{cccccc}
\hline Longitude & Latitude & Tilt Angle & Height & Aspect Ratio & Half Angle \\
\hline $\begin{array}{c}\theta \\
{[\mathrm{deg}]}\end{array}$ & $\begin{array}{c}\gamma \\
{[\mathrm{deg}]}\end{array}$ & $\begin{array}{c}\mathrm{h}_{\text {front }} \\
{[\mathrm{deg}]}\end{array}$ & {$\left[r_{\odot}\right]$} & $\kappa$ & $\alpha$ \\
\hline 4.3 & 1.8 & 22.0 & 0.48 & {$[-0.04,+0.07]$} & {$[-7,+13]$} \\
\hline
\end{tabular}

Tab. 10: GCS mean error values obtained from a sensitivity analysis for 26 CMEs performed by Thernisien et al. (2009).

half angle $\alpha$ and the tilt angle $\gamma$ can amount to one magnitude larger than the deviations of the longitude and latitude. This indicates that it is more difficult to determine the parameters half angle and tilt angle than the longitude and latitude for a GCS fit performed with coronagraph data.

\section{The modeller's experience:}

Since the fits are done by hand they depend to some extent on the modeller's subjective understanding of the observed CME white-light structure. It requires a certain experience and skill using and adjusting all six parameters in their reciprocal dependence to get an optimal match with the CME's white-light appearance (Thernisien et al., 2009). Thernisien et al. presented in 2009 a best practice which describes how to adjust the parameters to obtain a fit with a good agreement of the CME's white-light appearance.

\subsubsection{The Changing Spacecraft Positions}

During the time period of the STEREO mission both spacecraft are changing their position to each other. Since mission start in 2007 the separation angle increased until February 2011 when it reached its maximum of $180^{\circ}$. Then it decreased until 2015 (see Figure 20 and 21 in Section 3.2). For that reason the change of the spacecraft's separation angle results in changing conditions of observation which will be discussed in this section. It will be explained under which conditions it is possible to resolve a CME in 3-D from observations with two observers.

The three-dimensional recognition of a CME is possible with stereoscopic observations of at least two observers. The positions of the observers A and B and the object of interest $\mathrm{P}$ (e.g. the apex of the CME's leading edge) are shown in Figure 49 a). The observers A and B have a view on object $\mathrm{P}$ with their line of sight a' and b', respectively. A and B are separated by the angle denoted with $\gamma$ which scales with the distance c between both observers. This distance is called 'stereo base line' in the field of stereoscopy. The point $\mathrm{P}$ observed from vantage point $\mathrm{B}$ is projected into the plane of sky of the observer, see Figure 50. Its position is determined to $\left(\mathbf{x}_{\mathbf{B}}, \mathbf{y}_{\mathbf{B}}\right)$. With the observation from only one vantage point it is not possible to determine the position in the third dimension (here: $z_{B}$ ) along the line of sight b'. For that purpose a second observer is necessary to calculate the position of $\mathrm{P}$ on the line of sight b'. This is shown with the projected position of $\mathrm{P}$ in the plane of sky of observer $\mathrm{A}$ (here: $\left(\mathbf{x}_{\mathbf{A}}, \mathbf{y}_{\mathbf{A}}\right)$ ) which defines the position of $\mathrm{P}$ on the observer B's line of sight b'. Another projected position $\left(\mathbf{x}_{\mathbf{A}^{\prime}}, \mathbf{y}_{\mathbf{A}^{\prime}}\right)$ in the plane of sky of observer A would result in position $\mathrm{P} 1$ or $\mathrm{P} 2$, for instance. 


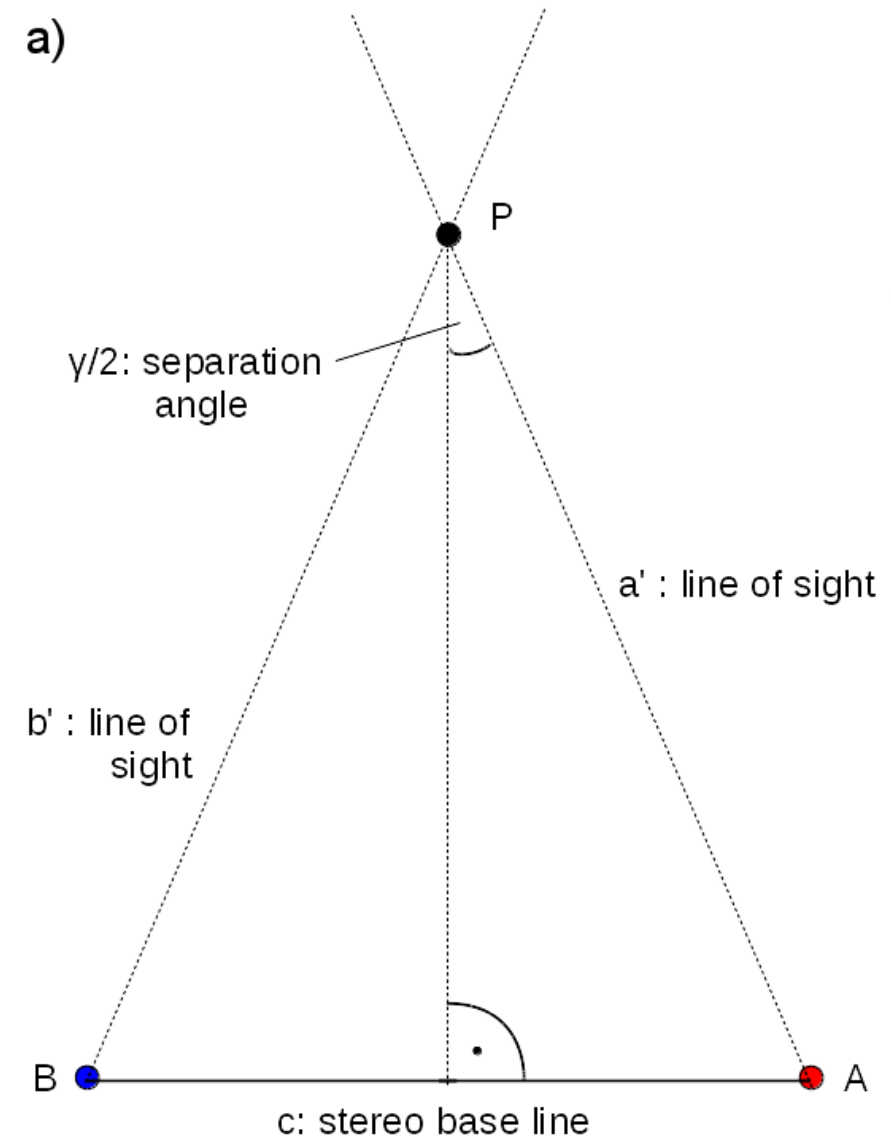

b)

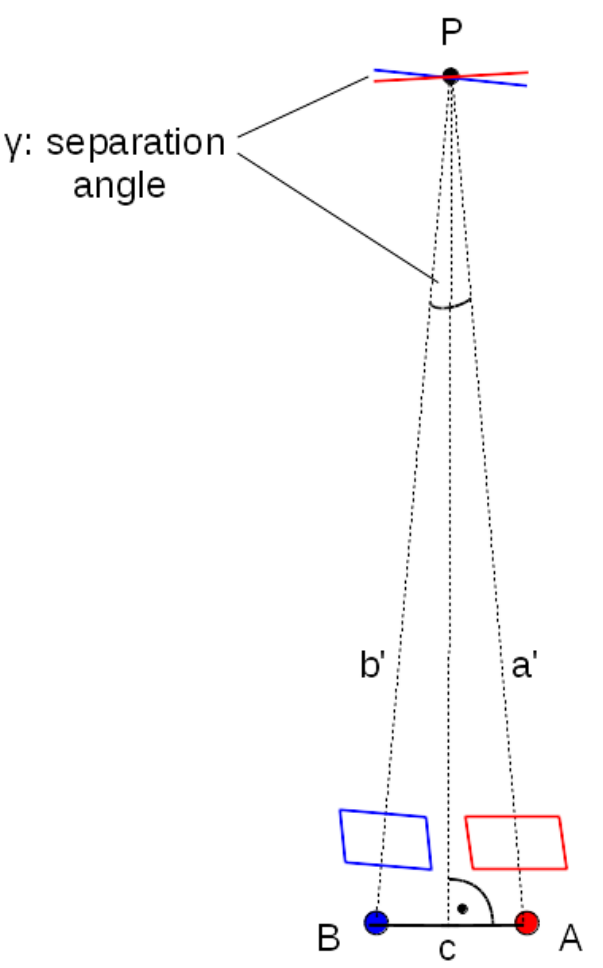

Fig. 49: a) Two observers $A$ and $B$ and the object of interest P. a' and b' denote the line of sights for each observer, which are separated by the angle $\gamma$. The distance between both observers is the stereo base line c. b) The stereoscopic observation of object $\mathrm{P}$ from two vantage points $\mathrm{A}$ and $\mathrm{B}$, which are separated by less than $10^{\circ}$. The planes of sky of both observers are shown in perspective with blue and red boxes at the bottom. The blue and red lines at the top present the planes of sky as seen perpendicular to the image plain and therefore reduced to one dimension. The angle between both planes of sky is the same as the one between observers A and B. This results in two similar projected views of the same object.

As mentioned at the beginning, the separation angle between both observers changes during the time period of the STEREO mission and with it the conditions of observation. The stereoscopic observation with a changing separation angle is discussed in this paragraph in relation to their limitations and uncertainties in the 3-D parametrisation of CMEs.

Separation angle $0^{\circ}-10^{\circ}$ :

The planes of sky of both coronagraphs become nearly congruent for small separation angles. This is shown in Figure $49 \mathrm{~b}$ ). As a consequence both STEREO/SECCHI/COR2 coronagraphs have roughly the same view of the CME, which is the case for the first two months in 2007. The CME appears at nearly the same position angle in both coronagraph images. A spatial resolution in 3-D is defective, because both planes of sky are almost congruent. The determination of the parameters in the third dimension, perpendicular to the plane of sky, is affected with a large uncertainty. This is explained in Figure 51. It is illustrated the determination of the position of point $\mathrm{P}$ in the third dimension, i.e. along the line of sight of observer B. The distance between observer B and point $\mathrm{P}$, named $z_{B}$, can be calculated by:

$$
z_{B}=\frac{c}{2 \sin (\gamma / 2)}
$$




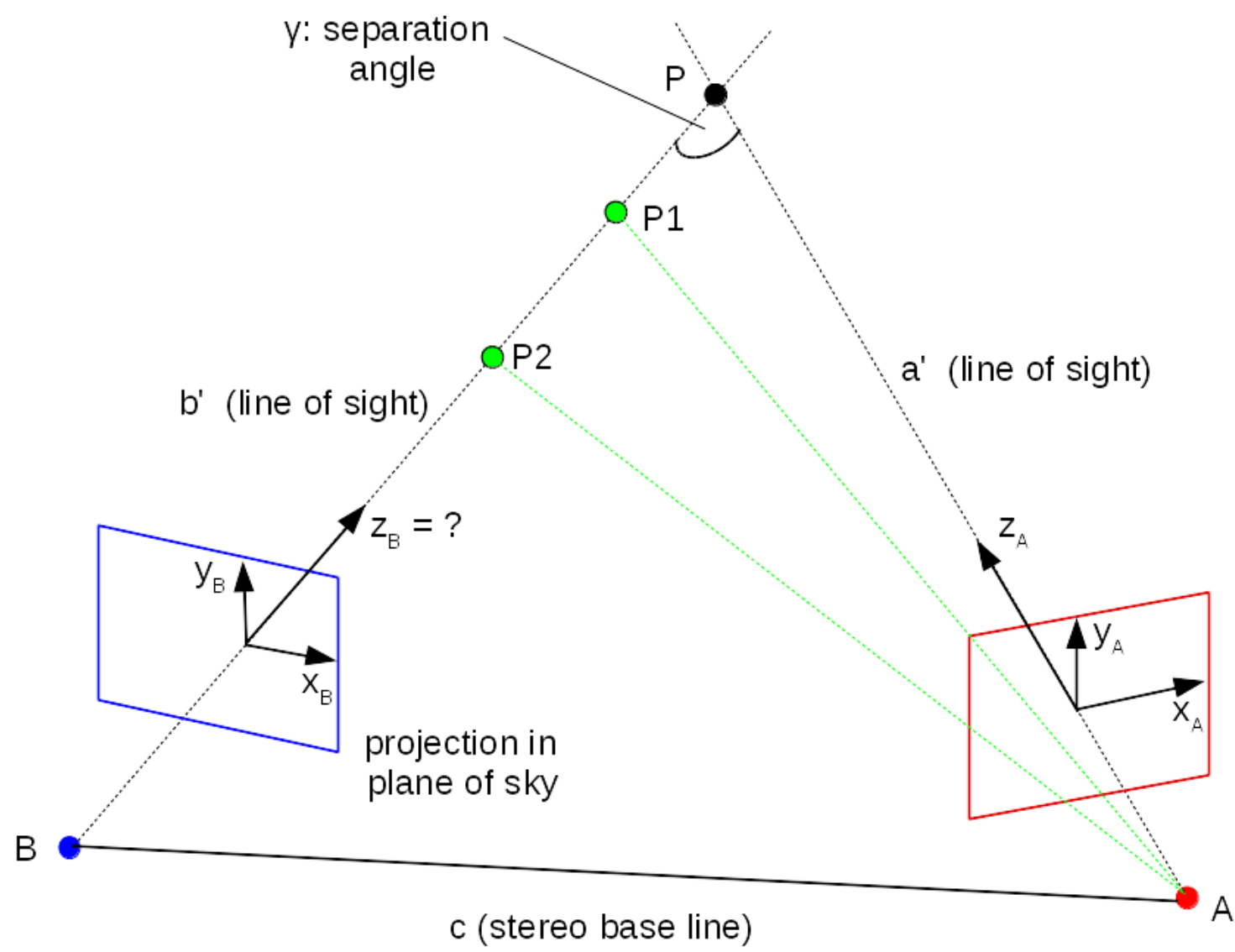

Fig. 50: A point $\mathrm{P}$ observed from vantage point $\mathrm{B}$ is projected into the plane of sky (blue) of the observer. It's position is determined to $\left(x_{B} / y_{B}\right)$. With the observation from only one vantage point it is not possible to determine the position in the third dimension (here: $z_{B}$ ). The projected position of $\mathrm{P}$ in the plane of sky of a second observer A (here: $\left(x_{A} / y_{A}\right)$ ) defines the position of $\mathrm{P}$ on the observer B's line of sight b'. Another projected position $\left(x_{A} / y_{A}\right)$ in the plane of sky of observer A would result in position P1 or P2, for instance.

with $c / 2$ half of the distance between both observers and $\gamma / 2$ half of their angular separation. The uncertainty $\Delta z_{B}$ of the position in direction of $z_{B}$ is indicated with a green arrow. This error depends on the separation angle $\gamma$. The error $\Delta z_{B}$ increases very strongly for small separation angles which is shown in Figure 52 with a plot of the function $z_{B}$. In contrast, the determination of the three-dimensional position of an object has its highest accuracy for two observers which are separated by $90^{\circ}$ in longitude. The function shown in Figure 52 has its minimum in uncertainty for this case.

This is in agreement with the findings from Inhester (2006) and Thernisien et al. (2009). Observations with a separation angle $>10^{\circ}$ result in two different views of the object and provide enough information for an unambiguous determination of the object's three-dimensional geometry.

\section{Separation angle $10^{\circ}-90^{\circ}$ :}

The angular separation between both spacecraft is large enough to resolve the CME well in 3-D from both 2-D images. Both spacecraft have different views on the CME. This is shown for a CME observed in November 2007 (Figure 44 in Section 5.4.2) and for another one in April 2008 (Figure 47 in Section 5.4.5). At this time the STEREO spacecraft were separated by $40^{\circ}$ and $50^{\circ}$, respectively. Thernisien et al. (2009) analysed with the GCS modeling technique the 3-D geometry 


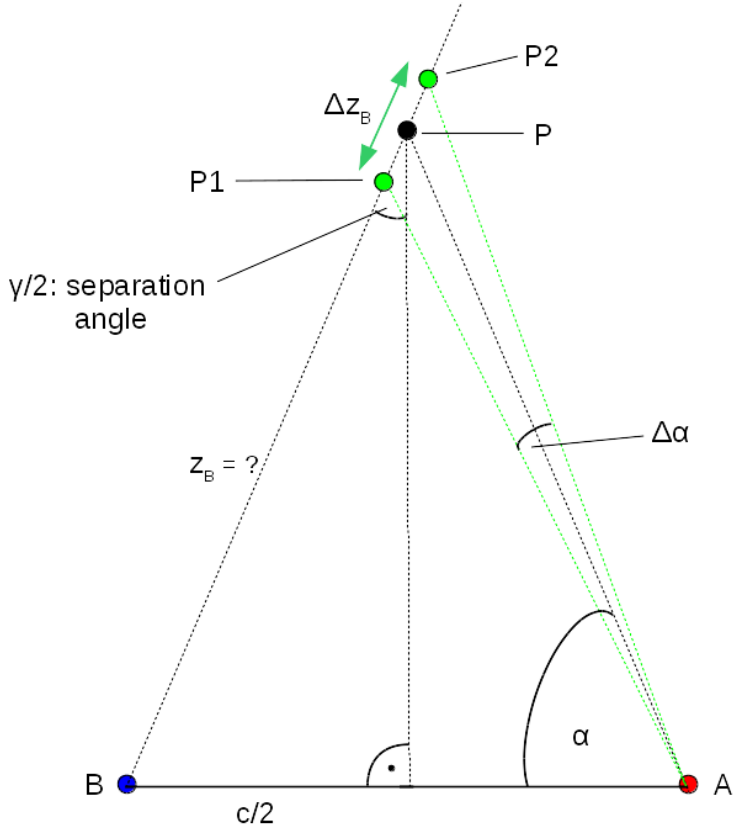

Fig. 51: An observer $B$ has a view on point $P$. The determination of the position of point $\mathrm{P}$ along the line of sight of observer $\mathrm{B}$ is illustrated. The distance between observer $\mathrm{B}$ and point $\mathrm{P}$, named $z_{B}$, can be calculated according to equation (5) with $c / 2$ half of the distance between both observers and $\gamma / 2$ half of their angular separation. The uncertainty $\Delta z_{B}$ of the position in direction of $z_{B}$ is indicated with a green arrow. The position of $\mathrm{P}$ on the observer B's line of sight is fixed by the view of observer $\mathrm{A}$.

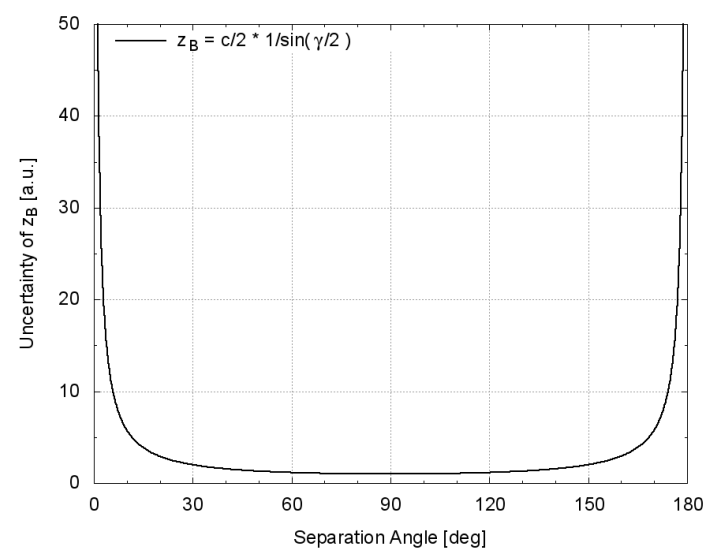

Fig. 52: The uncertainty in determining the position of $\mathrm{P}$ in direction of $z_{B}$ (Figure 51) depends on the distance between both observers, namely $c / 2$ and $\gamma$. The correlation, given in equation (5), is shown in this diagram. The uncertainty increases very strongly for a separation angle converging to $0^{\circ}$ or $180^{\circ}$. In contrast the accuracy has its maximum for an angular separation of $90^{\circ}$.

of 26 CMEs, which were observed with a separation angle from $40^{\circ}$ to $65^{\circ}$. They found no decrease of precision for their calculation of the 3-D GCS parameters.

\section{Separation angle $90^{\circ}$ :}

The situation of two observers which are separated by $90^{\circ}$ is presented in Figure 53 . The planes of sky of both spacecraft coronagraphs are also oriented by $90^{\circ}$ to each other (blue and red line at the top) and therefore provide the best conditions to resolve a CME in 3-D. Thernisien et al. (2009) stated, that the precision of the CME parametrisation with the triangulation method is maximal for a spacecraft separation angle of $90^{\circ}$, which is in agreement with the presented explanation in the previous paragraphs and Figure 52. An example of a CME observation for this case is shown in Figure 45 (Section 5.4.3) when both STEREO spacecraft were separated by nearly $90^{\circ}$.

A CME which is directed to one observer represents a special case in this situation of observation: A CME seen from STEREO-A as Halo CME appears for STEREO-B as limb CME in its field of view (FOV) and vice versa.

Separation angle $90^{\circ}-170^{\circ}$ :

The situation of CME observations and three-dimensional localisation during this period is comparable to the situation for separation angles ranging from $10^{\circ}$ to $90^{\circ}$. The planes of sky from both observers exhibit large separation angles and therefore a small uncertainty for $z_{B}$ (see Figure $52)$. 


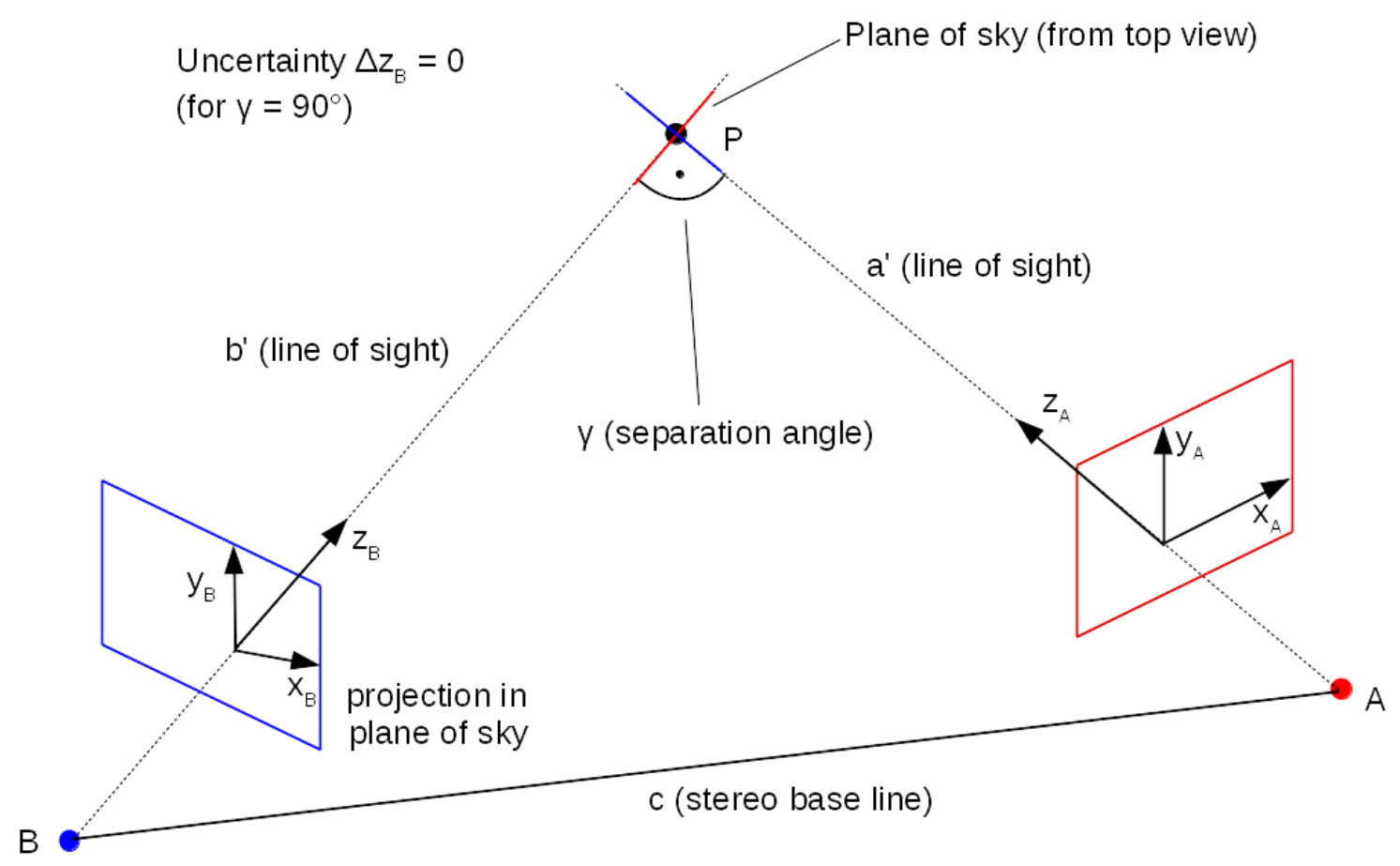

Fig. 53: The point $P$ observed from two vantage points $A$ and $B$ which are separated by $90^{\circ}$. The uncertainty for an observer to determine the point in direction $z_{A, B}$ perpendicular to its plane of sky is eliminated due to the observation of the second observer. The planes of sky of both observers are oriented perpendicular to each other which is indicated by the blue and red lines at the top.

Separation angle $170^{\circ}-180^{\circ}$ :

With the separation angle $\gamma$ between both spacecraft converging to $180^{\circ}$, the angle $\delta$ between both planes of sky decreases to zero. This is shown in Figure 54. The planes of sky from both coronagraphs are indicated with a red and blue solid line. Both planes of sky become congruent with decreasing angle $\delta$ and therefore the third dimension, perpendicular to the plane of sky, cannot be resolved very well for an object. The uncertainty to determine the extension of an object along the line of sight increases significantly (see Figure 52) when both spacecraft observe the CME from opposite directions. This was the case for the STEREO mission in February 2011.

This is particularly evident for a CME seen from STEREO-A as front side halo and from STEREO$\mathrm{B}$ as back side halo. This can result in a wrong determination of the CME's direction in longitude. A CME which occurred in February 2011 was observed as front-side or backside Halo CME from STEREO-A and -B, respectively. Due to these observation conditions in this case, first calculations resulted in a wrong direction of propagation for the Halo CME.

Millward et al. (2013) described a similar problem for coronagraph observations of Halo CMEs from one vantage point (e.g. SOHO/LASCO). The author discussed the "cone angle/radial distance ambiguity" for CME modeling with the cone model. For a Halo CME directed to the observer, the cone angle and the radial distance exhibit an inherent correlation. A CME visible in white-light appears with a certain expansion in the plane of sky and can either be fitted with a large radial distance and a small cone angle, or vice versa as shown in Figure 55. Applied to the GCS model this affects the parameters half angle and height. This leads to the "half angle/radial distance ambiguity" for two observers separated by $180^{\circ}$. 


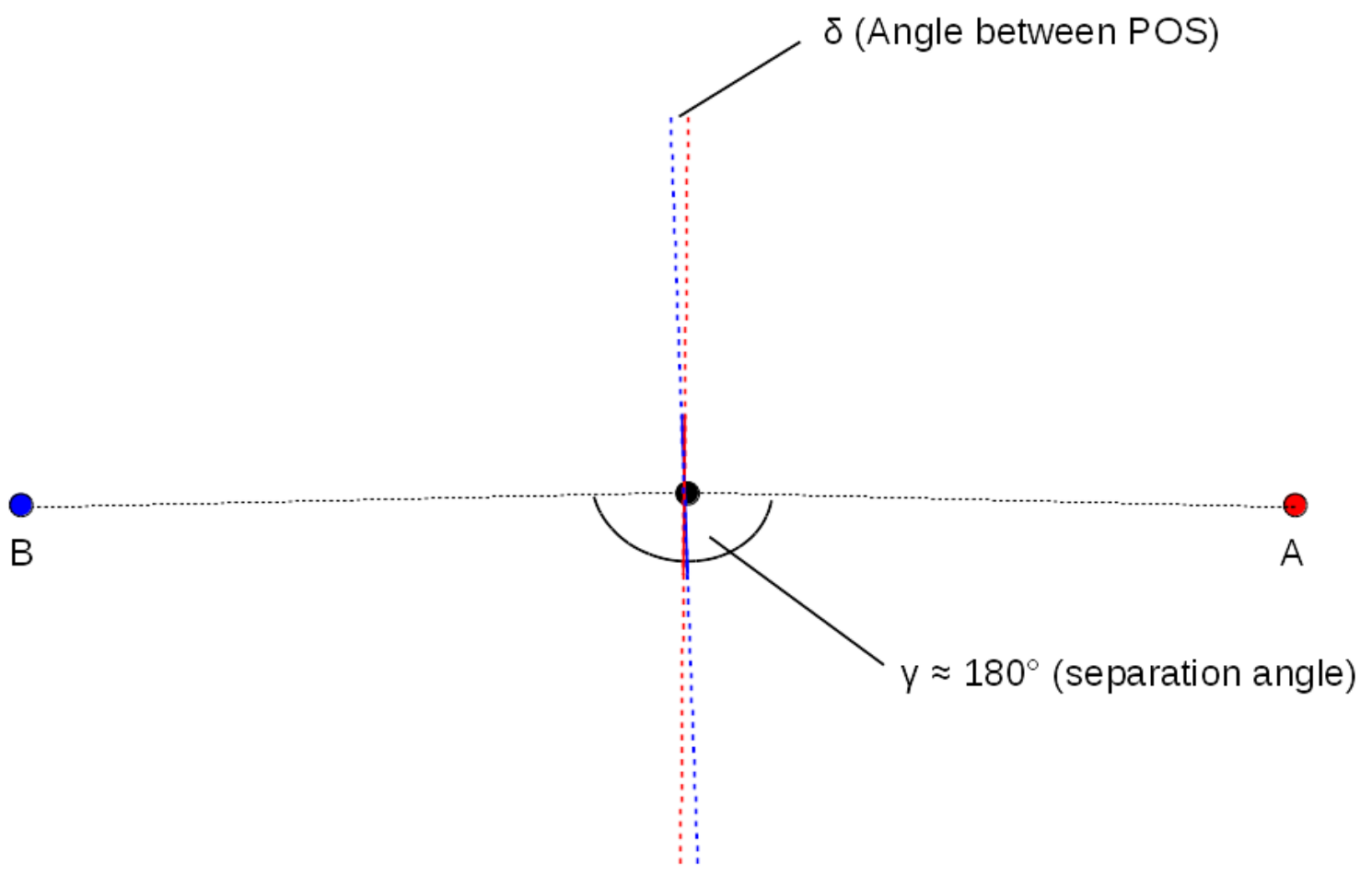

Fig. 54: Two spacecraft A and $B$ which are separated by nearly $180^{\circ}$ observe an object P. The angle $\delta$ between their planes of sky (blue and red solid lines) converges to $0^{\circ}$. Hence both planes of sky become congruent and the third dimension, perpendicular to the plane of sky, cannot be resolved very well for an object. The function shown in Figure 52 describes the increasing uncertainty for this case.

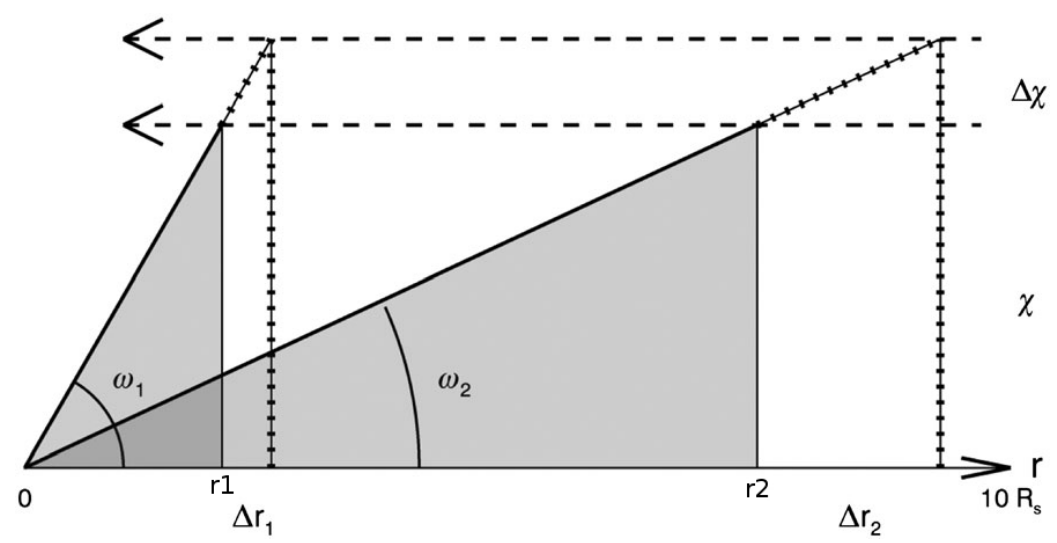

Fig. 55: The ambiguity between the cone half angle and radial distance for single point observed Halo CMEs. In this diagram the cone apexes are located in the Sun center at the origin of the x-axis which directs to Earth far away on the right. The white-light appearance of the CME's front can be modelled with a cone cross-section based on two different pairs of a cone angle and radial distance, labelled with $\left(r_{1}\right.$, $\left.\omega_{1}\right)$ and $\left(r_{2}, \omega_{2}\right)$. Both pairs of parameters yield to the same cross-section expansion $\chi$ but reveal different distances to the Sun center or the observer, respectively. From Millward et al. (2013).

\section{The GCS parameters:}

The changing conditions of observation have an influence on the GCS modeling of CMEs. Assuming that a CME is observed from one observer at a viewing angle of e.g. $20^{\circ}, 40^{\circ}$ or $60^{\circ}$ : It was not found within this work that the accuracy in determining the GCS parameters varies to a large 
extent with changing viewing angles. This was expected because the second observer with a large angular separation to the first one reduces the uncertainties in determining the GCS parameters with its second view. However, there are special cases, when the calculation of a GCS parameter is defective or cannot be calculated at all. These cases are discussed for the affected parameters half angle, tilt angle and height.

\section{Half Angle:}

A CME which is seen from both observers in edge-on view has its plane of symmetry almost coplanar with the ecliptic plane (Thernisien et al., 2009). An example for such a CME is shown in Figure 40 in Section 5.2.3 or the CME presented in Figure 61 in Section 6.4. In this cases the GCS tilt angle amounts to $\gamma \approx 0^{\circ}$. Hence it is not possible to determine the GCS half angle $\alpha$ which defines the length of the flux rope. In order to determine the half angle, a polar view or at least a vantage point from a few tens of degree out of the ecliptic is necessary (Thernisien et al., 2009). In other words, the half angle can be determined for CMEs which exhibit a flux rope with a tilt angle $\gamma \gg 0^{\circ}$.

\section{Tilt Angle:}

Another special case are CMEs which exhibit a more spherical shape. For these CMEs the half angle $\alpha$ converges to $0^{\circ}$. With that the GCS model turns into the ice-cream cone model (Fisher and Munro, 1984). As a consequence the tilt angle $\gamma$ becomes obsolete for this GCS configuration due to the sphere's rotational symmetry.

\section{Height:}

Problems can occur for the calculation of the GCS height or half angle, respectively. This is the case, as discussed in the previous paragraph, for observations of Halo CMEs with one coronagraph or two observers with separation angles of $\leq 10^{\circ}$ or $\approx 180^{\circ}$. This results in the "half angle/radial distance ambiguity" as shown in Figure 55.

\subsection{Identification of the CME's Source Region}

In order to identify the source region of an associated CME event the CME propagation was backtracked from the COR2 to the COR1 coronagraph's field of view and to EUVI data observed at $195 \AA$ and $304 \AA$ (Subramanian and Dere, 2001). The position angle in the COR1 coronagraph image of the propagated CME and the first point in time when the CME appears in the COR1 field of view gives a clue where the source region of the CME could be located on the solar surface. Running difference COR1 movies were used for a better CME tracking, these movies are provided by the Goddard Space Flight Center ${ }^{21}$. The EUVI data were searched for active regions, prominence eruptions, post eruptive arcades and for flares as indicator for a source region (Rust and Webb, 1977; Webb and Hundhausen, 1987; Harrison, 1995 and Tripathi et al., 2004). The backtracking of a CME observed on June 7th, 2011 at 08:08 UT with STEREO/SECCHI/COR2-A is shown in Figure 56. A flare, visible in COR1-A at 07:20 UT, and a prominence eruption in EUVIA $(304 \AA)$ at 06:56 UT indicate clearly the source region of this CME. The source region's location was determined with the Solar Weather Browser ${ }^{22}$ which was developed and is provided by the Solar Influence Data and Analysis Center (SIDC) at the Royal Observatorium of Belgium (ROB). For this CME the source region's (NOAA AR 11226) location was identified at $33^{\circ}$ Carrington longitude and $-23^{\circ}$ heliographic latitude. The uncertainty in estimation of the source region's location is given with $\pm 5^{\circ}$ referring to a $10^{\circ}$ grid of the solar weather browser. For comparison the GCS fit revealed a CME position of $33.5^{\circ}$ and $-14^{\circ}$ in longitude and latitude, respectively. The GCS fit for this CME is shown in Figure 57 (top row). The foot points of the GCS flux rope are displayed in the EUVI-A image in green. The white coloured plus signs in the EUVI-B image indicate that the foot points are located on the far side of the Sun behind the limb for this observer. This case shows a very good agreement between the CME modeling result and the CME's observed source region in the EUVI-A image.

\footnotetext{
${ }^{21}$ Goddard Space Flight Center: http://cor1.gsfc.nasa.gov/dailymov/

${ }^{22}$ Solar Weather Browser: http://sidc.oma.be/SWB/
} 


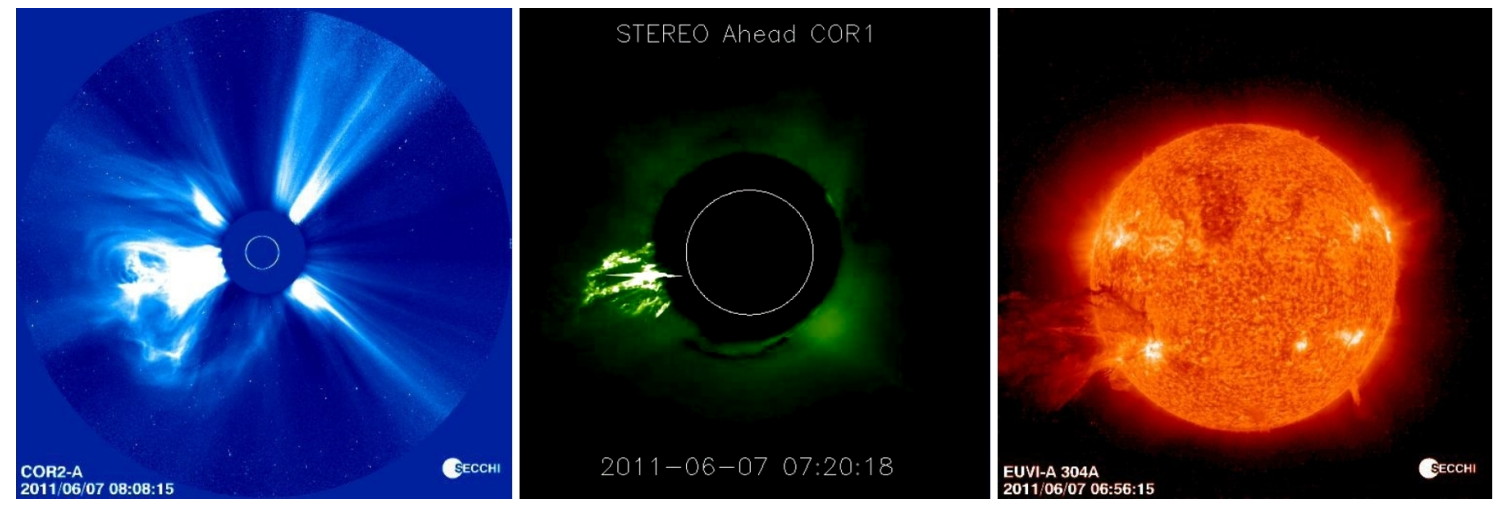

Fig. 56: A CME observed on June 7th, 2011 at 08:08 UT with STEREO/SECCHI/COR2-A (left) is traced back for the identification of its source region. A flare visible in COR1-A at 07:20 UT (center) and a prominence eruption in EUVI-A $(304 \AA$ ) at 06:56 UT (right) indicate the source region of this CME.
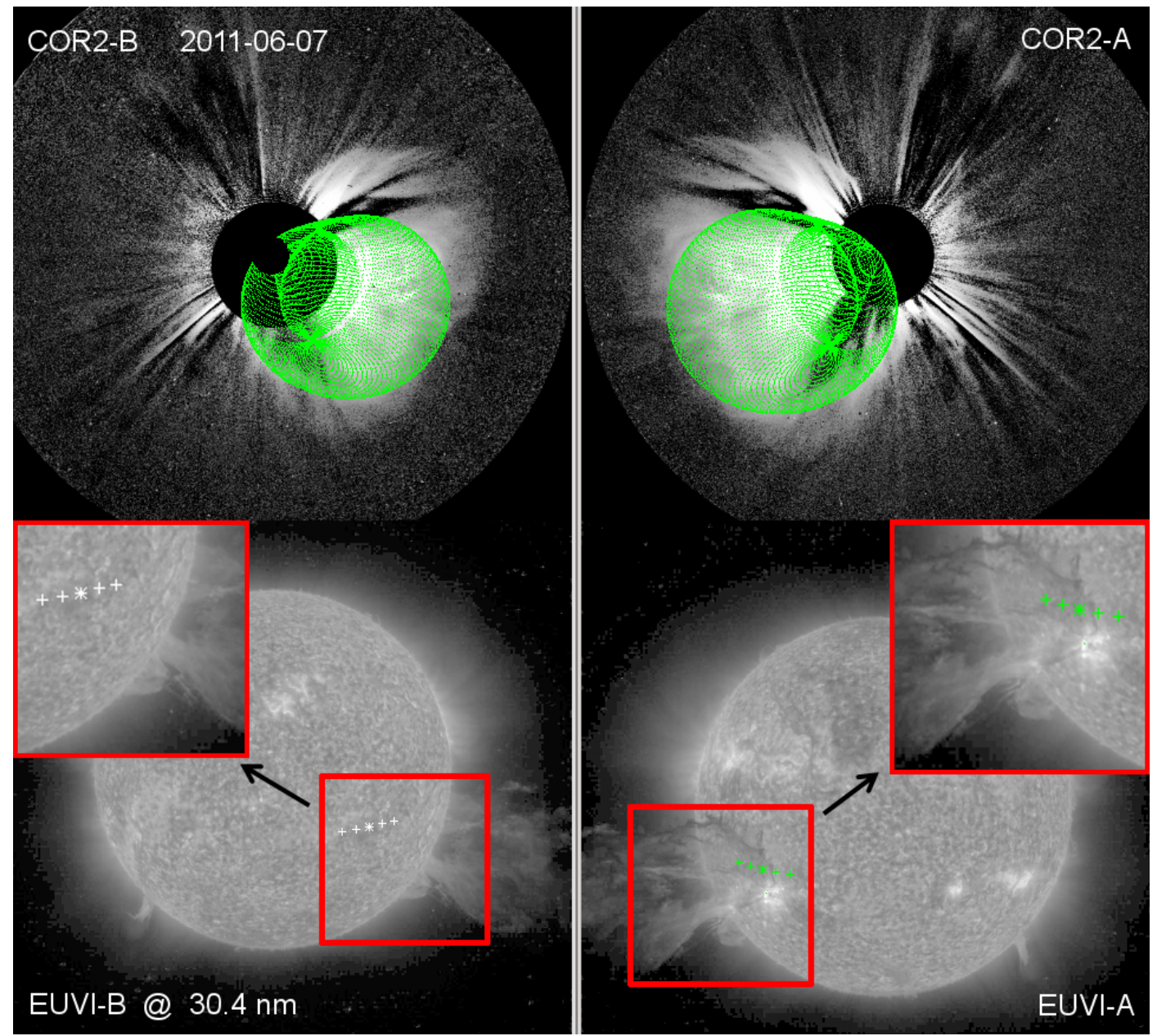

Fig. 57: The CME observed on June 7th, 2011 at 08:08 UT with STEREO/SECCHI/COR2 is fitted with the GCS modeling technique which is shown in green (top row). The foot points of the GCS flux rope are displayed in the EUVI-A image in green. The white coloured plus signs in the EUVI-B image indicate that the foot points are located on the far side of the Sun behind the limb. 


\section{Selected CME Classes and the Flux Rope Model}

The 'Best-of' CMEs, which are studied in this thesis, exhibit different 2-D morphologies in their coronagraph's white-light appearance. At first sight some of those different CME shapes might not correspond to the CME model of a three-part structure or the flux rope model for CMEs. Hence it is clarified in this chapter whether CMEs of different white-light appearances can be described with the GCS flux rope model and how these CMEs look like in terms of this model.

For this purpose a CME from each morphology class is fitted with the GCS modeling technique to determine its 3-D geometry. Four selected CME classes with the associated GCS CME fits are presented in this chapter. The remaining CME classes with their GCS fit results are summarised in the Appendix A.2. In the next step the approach of the electron density distribution from the GCS model (see Section 5.2.2) is applied to the CME's geometry in order to compute with the GCS ray-tracing code synthetic coronagraph images of each CME. Finally, these synthetic coronagraph images are compared with the original coronagraph images. This is presented in the following sections.

\subsection{CME Class 01 - Halo CME}

Halo CMEs are events which have a large expansion and do not show the typical shape of a flux rope because they propagate towards or away from the observer as shown on the right in Figure 58. The CME observed on May, 18th 2011 is not only visible as front side Halo by STEREO-A (top) but also as back side Halo from STEREO-B (bottom) because both spacecraft were separated by $\approx 180^{\circ}$ in longitude at this time. The corresponding synthetic coronagraph images are presented on the left. In a first step the CME's 3-D geometry was determined by applying the GCS modeling technique to the COR2 coronagraph data. In the next step the synthetic coronagraph images for this 3-D geometry were computed with a ray-tracing code taking into account the Thomson scattering of the derived electron density distribution. The parameters for the electron density and for the ray-tracing calculation are listed in Table 11 at the end of this section. The synthetic images describe very good the overall shape of the CME as well as its thin and bright leading edge. Also the dark cavity behind the CME's front is visible. As a side effect from image rendering the solar disk is unfortunately represented not by one but two small black circles located in the center. For this CME the flux rope structure of the GCS model is directed towards the observer (STEREO-A) and both legs are going backwards on the left and right of the front part as seen from STEREO-A and for STEREO-B vice versa.

\subsection{CME Class 06 - 'Fish'-CME}

In contrast to the largely expanded Halo CMEs a small and narrow CME of the 'Fish'-CME class is presented in Figure 59 on the right. The CME which occurred on July, 8th 2007 was observed with a faint 'Fish' -like structure which is suggestive of 'crossing legs' at the CME's bottom part. Only a very faint and narrow stripe of the 'Fish' structure is visible in these IDL processed images. This results from the image selection for the GCS modeling and the difference imaging of the data processing. The upper part of the CME is characterised by a circular bright structure with a faint front near the apex in the direction of propagation. The corresponding synthetic images which reflect very well the optical features of the original CME's white-light appearance are shown to the left. The flux rope is shown in the edge-on view with a tilt angle of $7^{\circ}$. The flux rope exhibits a faint and narrow bottom part which represent the legs. The circular cross section with a thin and faint CME front encloses the dark inner volume of the flux rope's tube section which is also visible in the original images. The parameters of the electron density $\mathrm{N}_{e}$ and the Gaussian width $\sigma_{i n}$ and $\sigma_{\text {out }}$ which yield to these computed images are listed in Table 11.

The interpretation of this structure hypothesised an edge-on view of a GCS-like CME with crossed legs. For a normal GCS-like CME the associated foot points lay on a straight line on the solar 

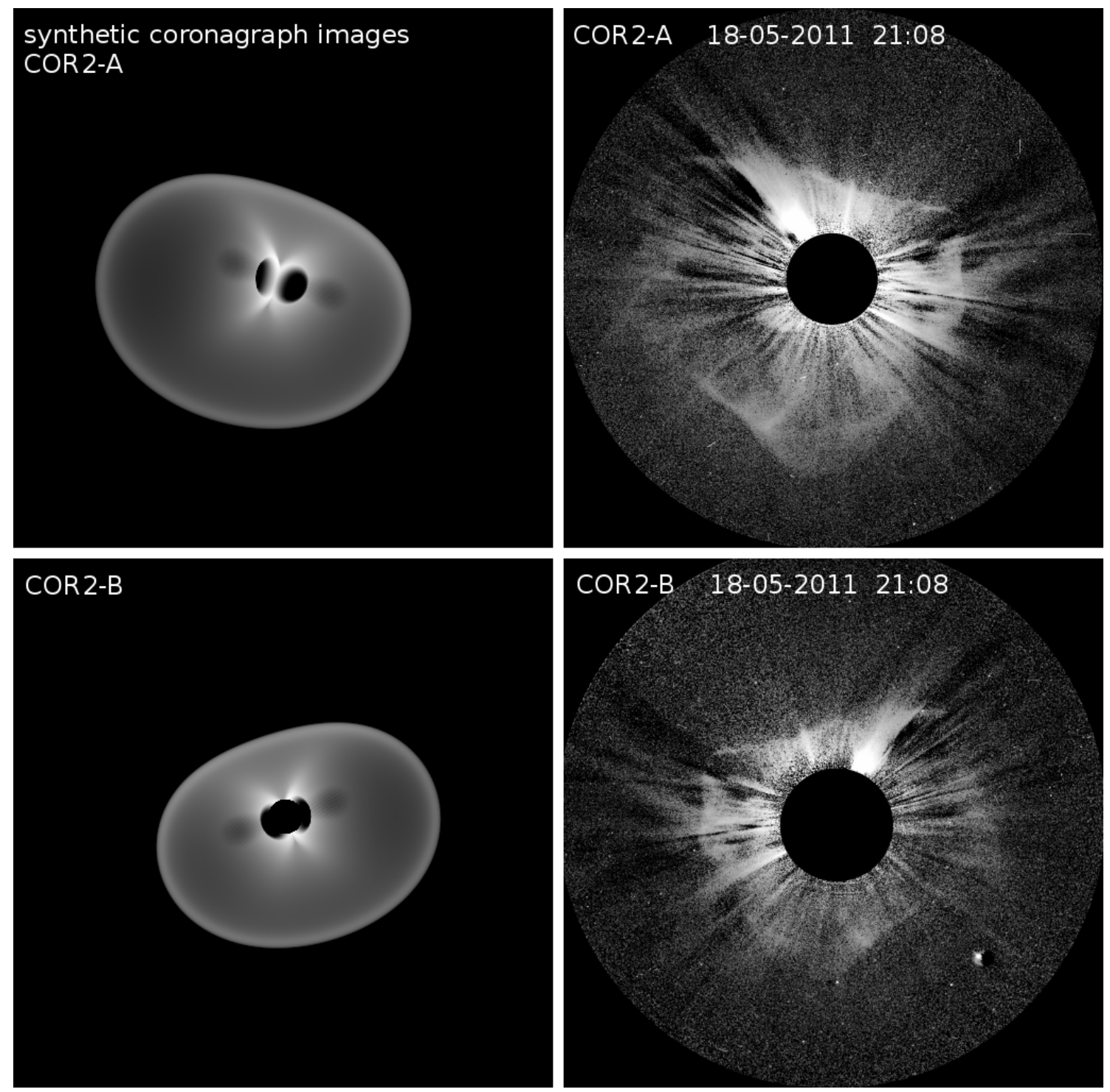

Fig. 58: Right: A halo CME observed on May, 18th 2011 which is propagating towards STEREO-A. The CME is also visible as (back side) Halo for STEREO-B because both spacecraft were separated by $\approx 180^{\circ}$ in longitude at this time. Left: Synthetic coronagraph images calculated with a ray-tracing code.

surface (see also Figure 39 in Section 5.2 and Figure 43 in Section 5.4). If the foot points of the legs are sheared against each other they compose a curved path on the solar surface leading to crossed legs for the flux rope structure above.

The assumption of a magnetic flux rope with footpoints which are sheared against each other was already published by Low in 2001. He discussed the magnetic effects of CMEs on the corona and described for magnetic flux ropes "the shearing of a coronal magnetic field by the displacement of its magnetic foot points in the photosphere". A sheared magnetic field constitutes one of the preconditions for CME origination and eruption. This is described in several CME models like the dynamo model or the tether-straining model (Aschwanden, 2006, Chapter 17.1 .2 and 17.1.5), respectively. Furthermore it was found from simulations by Török and Kliem (2003) that "an untwisted coronal flux tube which is sheared at its footpoints results in a loosely wound flux rope in equilibrium". 

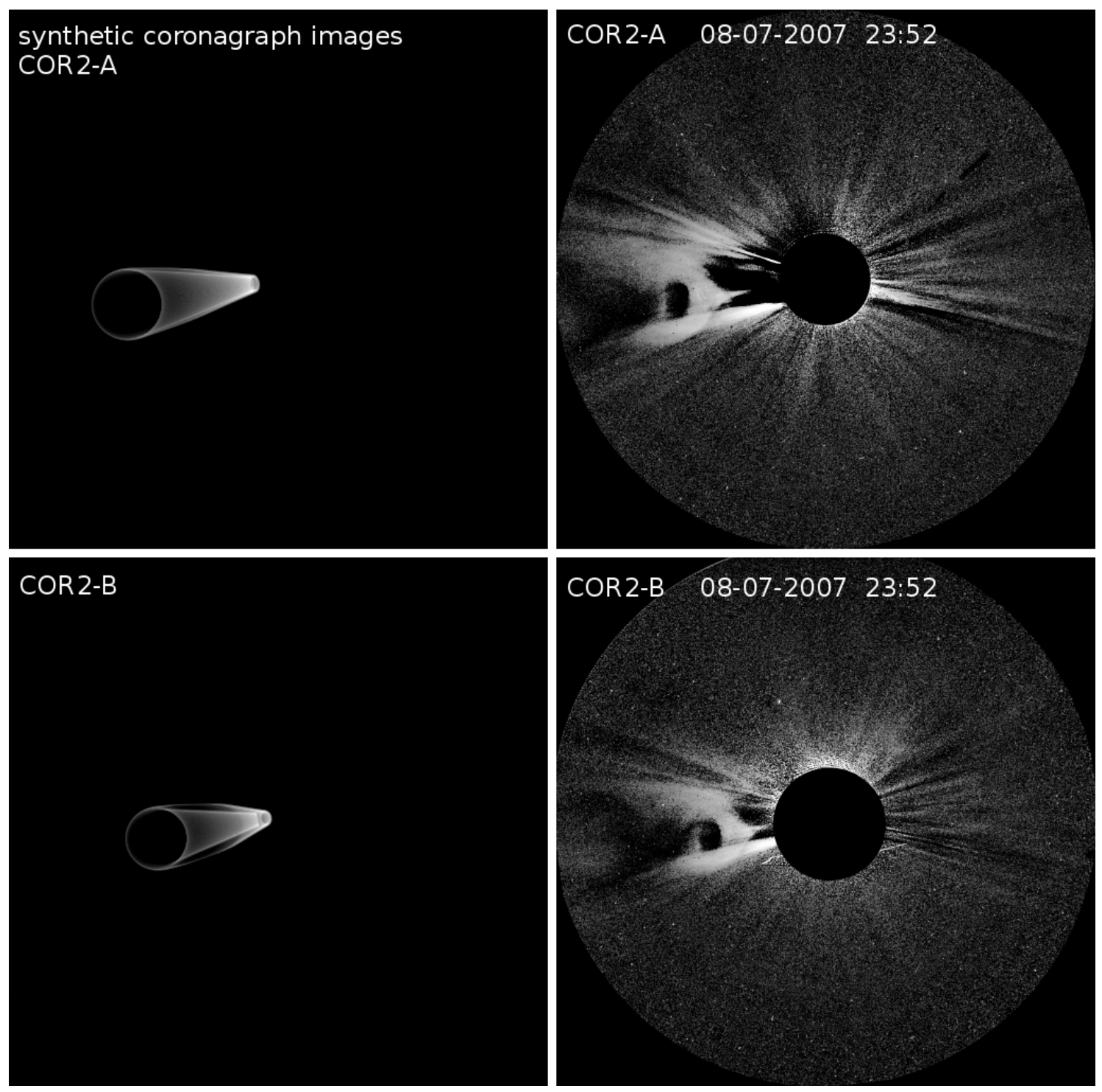

Fig. 59: Right: A 'Fish' type CME observed on July, 8th 2007 which shows a circular bright structure with a faint front near the apex in the direction of propagation and followed by a faint narrow stripe at its rear part. Left: The corresponding synthetic coronagraph images were calculated with a low electron density and a small Gaussian width to reproduce the faint CME structure and the thin leading edge. The parameters are specified in Table 11.

Another explanation for crossed legs leading to this white-light structure could be an outward propagating CME which is rotating around its axis oriented in direction of propagation. A $\left(180^{\circ}\right)$ rotation of the upper (and middle) CME part and its magnetic structure would result in a twist of the underlying legs of the flux rope structure. Indeed, a rotating CME was observed in June 2010 and reported by Vourlidas et al. (2011). 


\subsection{CME Class 07 - Classical CME}

The CME observed on May, 9th 2007 is presented on the right in Figure 60 and selected as an example for a 'classical' CME with a bright appearance, a clear structure and curved front. The corresponding synthetic coronagraph images are shown on the left which reflect very well the CME's overall shape. Furthermore the bright leading edge followed by the dark cavity is reproduced and matches the observations. The flux rope is oriented as edge-on view with one leg directly visible to the observer and the other one covered on the far side. The parameters describing the electron density distribution are provided in Table 11.
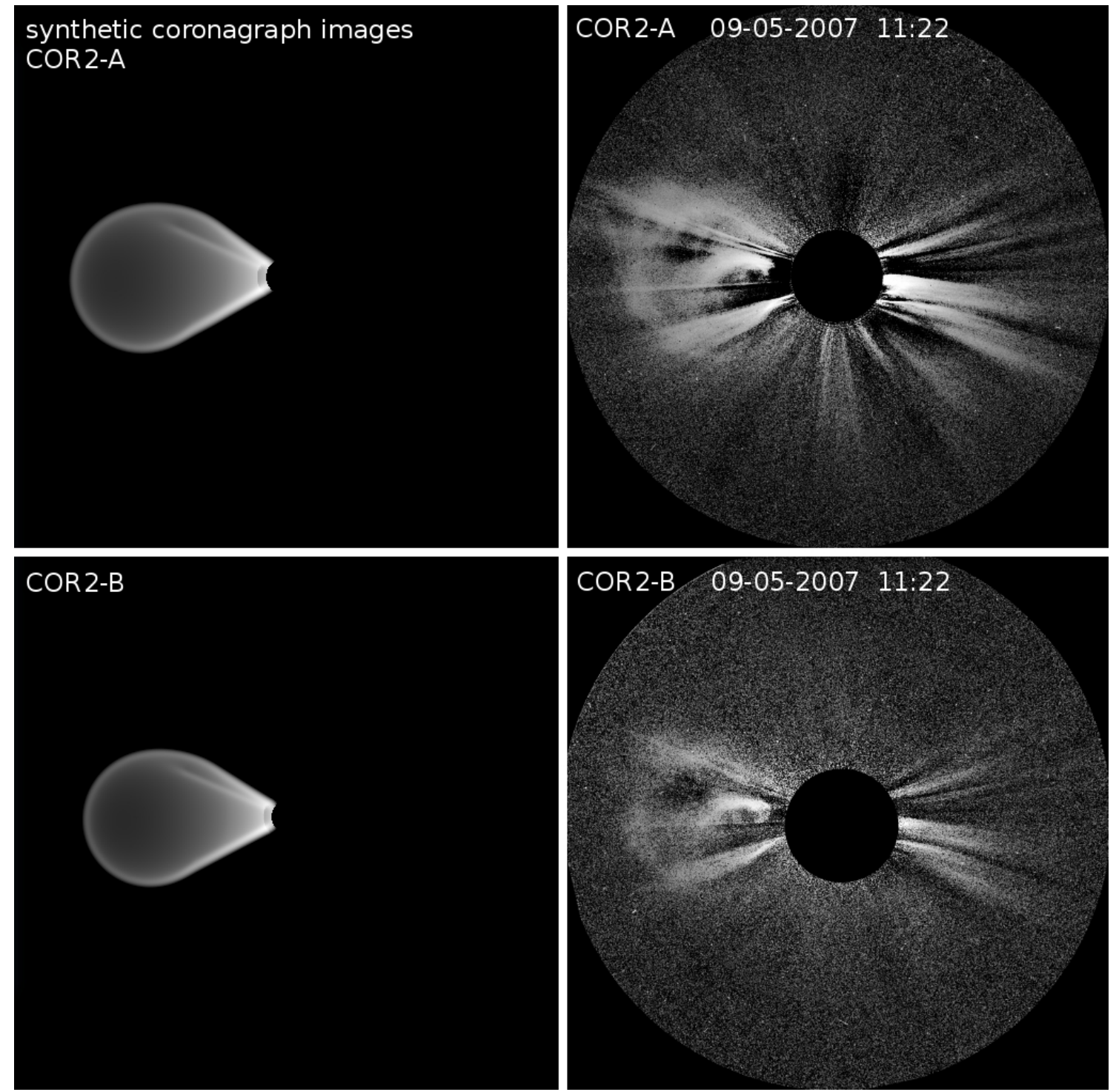

Fig. 60: Right: A 'classical' CME observed on May, 9th 2007 showing a circular bright front followed by a dark cavity. Left: The synthetic coronagraph images reproduce very well the CME's overall shape, its bright curved front and the dark cavity behind the leading edge. The parameters which describe the electron density distribution for the synthetic images are given in Table 11. 


\subsection{CME Class 09 - 'Laurel Wreath' CME}

The CMEs of the 'Laurel wreath' CME class show a bright ring structure as upper part however with a faint or missing front at the apex of the leading edge. This results in a white-light appearance similar to the shape of a laurel wreath enclosing a dark circular area in the middle. An example shown on the right in Figure 61 is presenting such a CME observed on January, 29th 2008. The
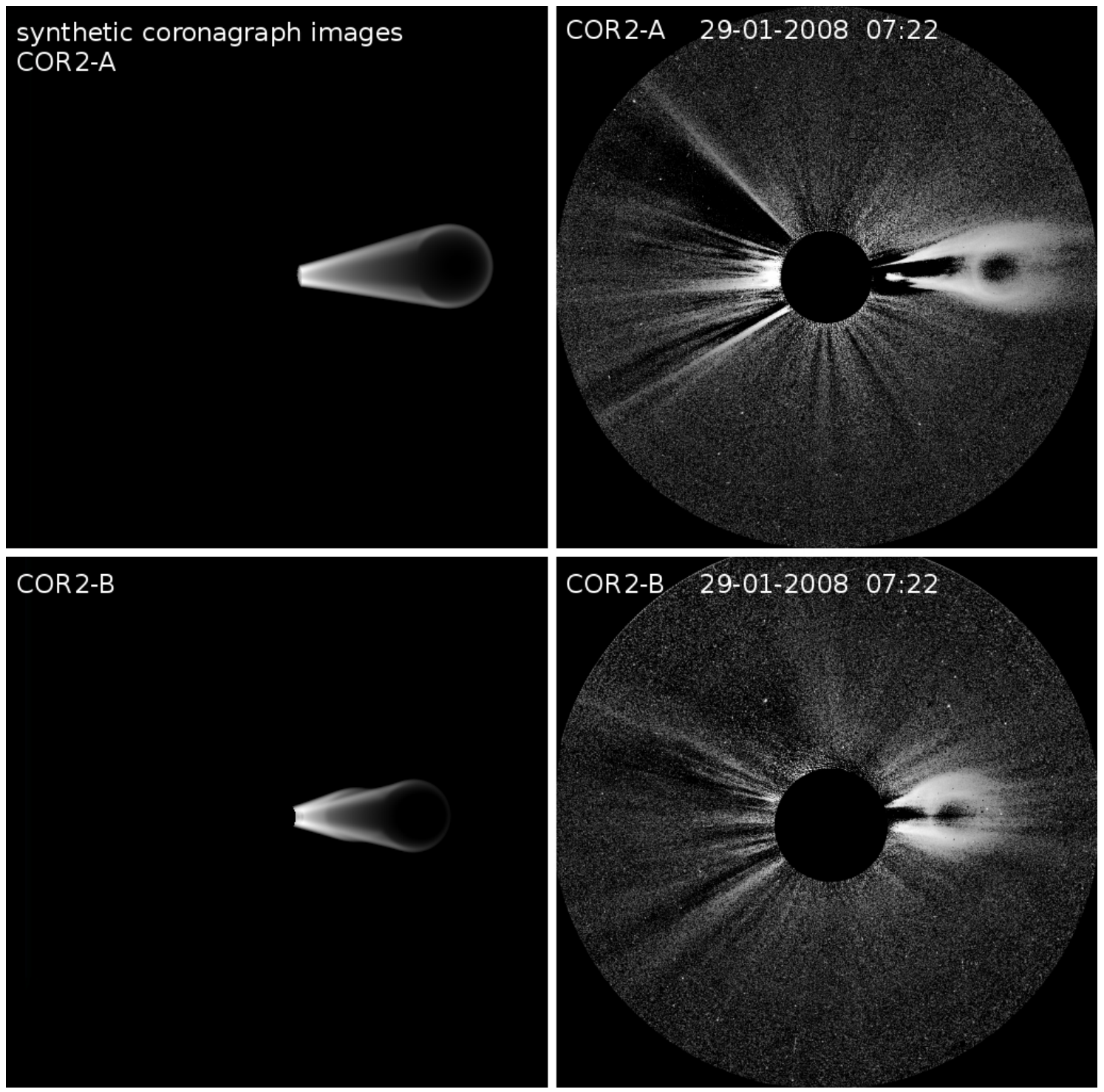

Fig. 61: Right: A 'Laurel wreath' type CME observed on January, 29th 2008 showing a circular bright structure. The CME is reminiscent to a laurel wreath with the missing bright front at the middle of the leading edge which would complete the ring structure. Left: The corresponding synthetic images reflect the observed circular white-light structure which encloses a dark void in the middle. This and also the faint cone-like bottom part is reproduced by a ray-tracing code applied on a low electron density distribution and a small Gaussian width for the electron profile. The parameters are listed in Table 11.

CME's bottom part exhibits a thin cone-like shape. From GCS modeling and Thomson scattering calculations synthetic coronagraph images were computed for this CME with a ray-tracing code. The synthetic images on the left in Figure 61 reflect the overall shape of the CME well. The thin ring-like structure of the upper part is visible and also the almost missing front at the center of the leading edge (COR-2B, bottom left). The dark void in the middle of the 'laurel wreath' 
structure and also the faint cone-like bottom part can be reproduced by a low electron density and a small Gaussian width of the electron density profile. The parameters for the Thomson scattering calculations are provided in Table 11. The GCS flux rope is oriented in edge-on view (see also Figure 38 in chapter 5.2.1) with a tilt angle of $5^{\circ}$ similar to the 'Fish' or 'Ring' CME class.

\begin{tabular}{cccccll}
\hline CME date & \multicolumn{6}{c}{ Parameter for synthetic coronagraph images } \\
dd.mm.yyyy & LOS & LOS & LOS & $N_{e}$ & $\sigma_{\text {in }}$ & $\sigma_{\text {out }}$ \\
& Range Min. & Range Max. & No of Points & & & \\
\hline 18.05 .2011 & -15 & 20 & 1024 & 200 & 0.4 & 0.1 \\
08.07 .2007 & -5 & 5 & 256 & 200 & 0.005 & 0.1 \\
09.05 .2007 & -10 & 10 & 128 & 400 & 0.2 & 0.2 \\
29.01 .2008 & -5 & 5 & 128 & 200 & 0.15 & 0.15 \\
\hline
\end{tabular}

Tab. 11: The modeling parameters which were used to compute the synthetic coronagraph images with the ray-tracing code from the GCS modeling technique. These parameters are from left to right the minimum and maximum range of the line of sight and their number of points for image rendering. $\mathrm{N}_{e}$ is the factor for the electron density and $\sigma_{\text {in }}$ and $\sigma_{\text {out }}$ represent the Gaussian width of the electron density profile inside and outside the GCS surface (see also Figure 38 in Chapter 5.2.1).

As shown by these studies the most different appearances and morphologies of CMEs observed with coronagraphs can indeed be described with the GCS flux rope model. Their different morphologies can be fitted with the GCS modeling technique resulting in a 3-D flux rope geometry. The synthetic coronagraph images which were generated from Thomson scattering calculations confirm their 3D flux rope geometry. The analysis of the CME classes in conjunction with their corresponding modeling results suggest that the different CME appearances can be grouped in small-scale, largescale and 'other' CMEs. Small-scale CMEs like the 'Laurel wreath', 'Fish' and 'Ring' -type CME can be best reproduced with the GCS model in edge-on view and a tilt angle near $0^{\circ}$. In this case the flux rope is oriented parallel to the solar equator. The CMEs of the classes 'classical', '(partial) Halo' and 'diffuse' CME represent large-scale CME events. Most of their different shapes, which are visible in the coronagraph images, are most likely due to projection effects as supposed by Schwenn (2006). This is also shown in Figure 44 and 47 in Section 5.4 for two CMEs observed in November 2007 and April 2008. These CMEs exhibit different white-light appearances in both coronagraph images which are observed from two observer separated by $40^{\circ}$ and $50^{\circ}$, respectively. The CMEs of the 'distorted' CME class show a distortion in their leading edge. For those CMEs it was possible to reproduce the overall shape aside from their deformed front. The GCS model assumes a circular curved CME front and therefore does not allow to modify the CME's leading edge. An example of a distorted CME and CMEs of the other classes are shown in the Appendix A.2 together with the corresponding synthetic coronagraph images.

\subsection{Comments and Comparison to the Morphology Study by Vourlidas (2013)}

The connection between the different CME morphologies observed in coronagraph images and the flux rope model was also analysed by Vourlidas et al. (2013) but with another approach and with a focus on single vantage point observations from SOHO/LASCO coronagraphs.

For their examination they revised the LASCO/SOHO CME catalogue and identified a 'new type' of a CME morphology consisting of two fronts. The outer one appears faint in brightness with diffuse material trailing behind. The second inner front is the bright leading edge from the wellknown CME's three-part structure. Therefore the authors extend this description to a five-part 
CME structure. The faint front is interpreted as shock front ahead of a fast propagating CME. This is also visible in coronagraph observations presented in this work. The CME observed on April 26th, 2008 is discussed in Section 5.4.5 and depicts such an example with a faint front and diffuse material following behind. The second aspect of their work gives strong indications in order to clarify 'where is the flux rope structure located in the CME's white-light appearance?'. In contrast to this work they implemented MHD simulations in order to find out which CME white-light features from coronagraph images can be reproduced with the approach of an erupting 3-D flux rope. With the calculation of the CME's morphology for several viewing angles they recreated both CME fronts in a very good agreement to the corresponding actual LASCO observations. As a second result they verified the CME three-part structure with the cavity as an erupting flux rope. Thereby their analysis and this thesis confirm the approach of the flux rope model for CMEs. 



\section{Statistical Results from Geometrical Fitting and Param- eter Analysis}

Several different CMEs, which can be fitted and described with the GCS flux rope model, were presented in the previous two chapters. It was shown that the CME's 3-D structure can be well determined from stereoscopic coronagraph images with the GCS modeling technique. Based on these findings this method is applied to all 241 CMEs of the 'Best-of' CME list. The modeling allows to analyse the 3-D geometry of a large number of CMEs in terms of the flux rope model, the results are presented and discussed in this chapter. The study covers CMEs occuring during time periods of low solar activity as well as events which occured at times with increasing solar activity. A study on the 3-D geometry of CMEs with the GCS modeling technique for events occuring over a five year long period was not yet performed to this extent until today. It is investigated, if CMEs of different size and geometry occur during different times of solar activity.

\subsection{Introduction}

As described in Chapter 4 the CME study started with the generation of an overall CME list resulting from the inspection of the STEREO/SECCHI/COR2 observations. An overview about this CME list and some statistical results about the occuring CME rates are given in the next section before the analysis of the 'Best-of' CMEs is presented.

\subsubsection{The Overall CME List}

Within the time period January 2007 until December 2011, 1071 CMEs were identified (judged visually) in the white-light synoptic movies of STEREO/SECCHI/COR2-A and -B observations. These movies are provided at the SECCHI website by NRL ${ }^{23}$.

This set of CMEs constitutes the overall CME list and contains additional information for each CME event like an internal CME number, the CME's position angle (PA) for each coronagraph observation, the positions of the spacecraft at CME detection time, the separation angle between both spacecraft and comments for the visibility of the CME white-light structure. In addition it is provided if the CME is a (partial) halo event for one of the STEREO spacecraft or if it is a back side CME in relation to the Earth's position. Due to the size of this list it is published in an online CME database ${ }^{24}$ where a more detailed explanation for this table is given.

In order to investigate the characteristics of the established overall CME list the monthly CME rate of this list was compared with the monthly CME rate obtained from the SOHO/LASCO CME catalogue published by Yashiro et al. (2004). For this comparison SOHO CME events with an angular width larger than $45^{\circ}$ were chosen because smaller and fainter structures did not fulfill the identification criteria for generating the overall CME list. The monthly CME rate of these SOHO CME events, the monthly CME rate of this overall CME list and the monthly smoothed sunspot number (SSN) obtained from the Solar Influence Data and Analysis Center (SIDC) at the Royal Observatory Belgium ${ }^{25}$ (ROB) are shown in Figure 62 a). The monthly sunspot number (SSN) was consulted as an indicator for the overall solar activity. The comparison of the monthly CME rate from the overall CME list (blue) and the CME rate derived from the SOHO/LASCO CME catalogue (green) show a very good agreement for large-scale CMEs. The monthly CME and SSN rates (red) show generally similar trends but no exact conformity. The CME and the sunspot monthly rates increase between September 2009 and March 2010 by a factor of three to four and remain on a high rate in the following months. This rise can be interpreted as the start of increased solar activity indicating the next solar maximum which was expected around 2013-2014. This is also recognisable in diagram b) of Figure 62 where the sunspot numbers per month are shown for solar cycle 23 and 24. It is striking that in 2009 the CME rate keeps constant (at about $10 /$ month for SECCHI and 6/month for LASCO) for several months although the corresponding

\footnotetext{
${ }^{23}$ COR2 synoptic movies: http://secchi.nrl.navy.mil/sccmovies

${ }^{24} \mathrm{CME}$ database: www. affects-fp7.eu/cme-database.

${ }^{24}$ SOHO/LASCO CME Catalogue: http://cdaw.gsfc.nasa.gov/CME_list/ published by Yashiro et al. (2004)

${ }^{25}$ Solar Influences Data Analysis Center at Royal Observatory Belgium: http://sidc.oma.be
} 

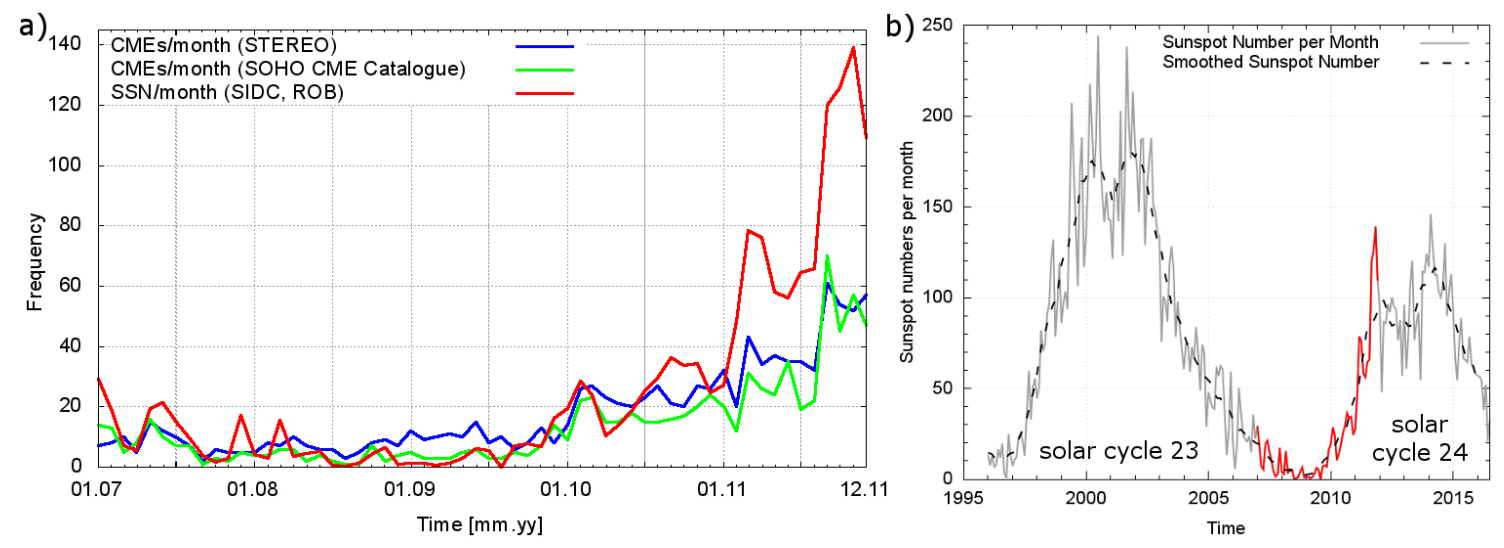

Fig. 62: a) CMEs per month observed with STEREO (blue) and registered in the overall CME list, monthly rate of CMEs with an angular width $\geq 45^{\circ}$ (green) derived from the SOHO/LASCO CME Catalogue $^{27}$ and the monthly smoothed sunspot number (SSN) from SIDC, ROB (red). Diagram b) presents the sunspot numbers per month of solar cycle 23 and 24 coloured in grey and for comparison with diagram a) from 2007 until 2011 in red. The smoothed SSN per month from 1996 to 2016 is given in black (dashed).

SSN fluctuates around zero (Bosman et al., 2012). "The difference between these two rates can be explained with decaying active regions of less intense magnetic flux remaining unidentified as sunspots but remaining as possible sources of CMEs, in agreement with what has been proposed by Cremades and Bothmer (2004)."

It should be considered that both CME catalogues differ regarding to the vantage points of the spacecraft. While on the one hand SOHO observes the Sun at a fixed vantage point with one spacecraft on the other hand STEREO moves around the Sun with different vantage points and positions related to each other. The second difference is owed to the fact that both lists with manually identified CMEs depend on the scientist's subjective ability of CME identification.

\subsubsection{The 'Best-of' CME List}

On the basis of the overall CME list a 'Best-of' CME list ${ }^{28}$ with 263 events was established based on the CME's clear morphology (judged visually) in the STEREO/SECCHI/COR2 images. No further selection limitations were applied like the angular width of a CME or if its topology is similar to a flux-rope or to a GCS like shape. This list constitutes the data set of CMEs which are studied in detail in this chapter. The subjects of the analysis for each event comprise the 3-D geometry, orientation and position of the CME in space. The parameters which describe these CME characteristics were determined with the GCS modeling technique. The 'Best-of' list contains for each event the basis parameters date, time and position angle of the coronagraph observation as a 'fingerprint' for re-identification in other catalogues. In addition the spacecraft position data from STEREO in relation to Earth position are listed for the time when the CME occurred in the STEREO/SECCHI/COR2 field of view.

\subsubsection{The GCS Modeling Results List}

The GCS modeling technique was applied to 241 CMEs of the 'Best-of' list. There was no fit possible for 22 CMEs of the 'Best-of' list because the CME was very good visible in COR2-A but only poorly visible in COR2-B or vice versa. Furthermore for some CME events there are no FITS files available which are needed for fitting.

\footnotetext{
${ }^{28}$ The 'Best-of' CME list is shown in Table A.4 in the Appendix.
} 
The list with the GCS modeling results ${ }^{29}$ comprises the following data:

- The time-stamp (date and time in UT) when the CME was best visible in the COR2 field of view. The FITS files were taken of this time for the GCS fit.

- The six determined GCS fit parameters (Longitude, Latitude, Tilt Angle, Height, Aspect Ratio, Half Angle) which describe the CME according to the GCS model.

- Flags which provide additional information about the event and the fit (Halo CME, missing FITS files, CME exhibits distorted leading edge, fit of a 'classical' CME).

A visualisation of the numerical results is provided for each CME of the GCS modeling results list in the CME online database at ww . affects-fp7 . eu/cme-database. The STEREO/SECCHI/COR2 images showing the CMEs can be found from the menu 'Database' in the sub-menu 'GCS modeling results' via a link at the CME numbers. In addition the COR2 images are shown with the GCS wireframe from fitting, similar to the Figures in this thesis.

\subsection{GCS Modeling Results}

The selected CME events from the 'Best-of' CME list were analysed and fitted with the GCS modeling technique as described in Section 5.4. The GCS modeling was applied to $241 \mathrm{CME}$ events observed between 2007 and 2011. The fitting yields to various results which are presented in this section in detail. These results illustrate what typical shape, position and orientation can be expected for CMEs observed near Sun between 10 and $20 r_{\odot}$.

\subsubsection{Carrington Longitude}

The position of the modelled CME flux rope is fixed by the Carrington longitude and the heliospheric latitude as described in Section 5.2. There is no physical reason that CMEs propagate in preferred longitudinal directions away from Sun due to its rotation symmetry. Hence it is expected that the distribution in Carrington longitude of all modelled CMEs is uniformly distributed. This is confirmed by the results and therefore a diagram of the CME's longitude vs. time is omitted. In his study about 26 CMEs, Thernisien et al. (2009) also found that the CMEs are distributed in almost all directions of longitude.

\subsubsection{Heliospheric Latitude}

The occurrence of CMEs at different latitudes is discussed in this paragraph. The CME's direction in latitude calculated from the GCS modeling refers to the apex of the CME's leading edge. Figure 63 presents the latitude of the apex projected on solar surface of the modelled flux rope for 241 'Best-of' CMEs plotted vs. time. The results of the GCS latitude show a different distribution for two periods of time. For CMEs occurring between January 2007 and January 2010 the GCS apex of the CME's leading edge was found for most cases at lower latitudes below $\pm 20^{\circ}$. From February 2010 and later CMEs occur at higher latitudes, up to $\pm 60^{\circ}$. This jump to higher latitudes for CMEs at the beginning of a solar cycle is in agreement with the latitude behaviour of occurring CMEs of the last solar cycles, for instance between 1997 and 2000 (end of solar cycle 22 and start of 23), Gopalswamy et al. (2003). Compared to the results of Gopalswamy for observed (not fitted) CMEs with SOHO/LASCO (Figure 64) the jump to higher latitudes is more distinctive and clearly visible in the GCS latitude fit results. Similar to the asymmetric occupation of CMEs in the latitude belt above $40^{\circ}$ solar north (see GCS latitude results in Figure 63) an asymmetric occupation can be found in the southern hemisphere beyond $-60^{\circ}$ (Figure 64). Therefore this asymmetry affects the opposite hemisphere in the previous solar cycle (Gopalswamy et al., 2003).

\footnotetext{
${ }^{29}$ The list with the GCS modeling results is shown in Table 14 in the Appendix.
} 
a)

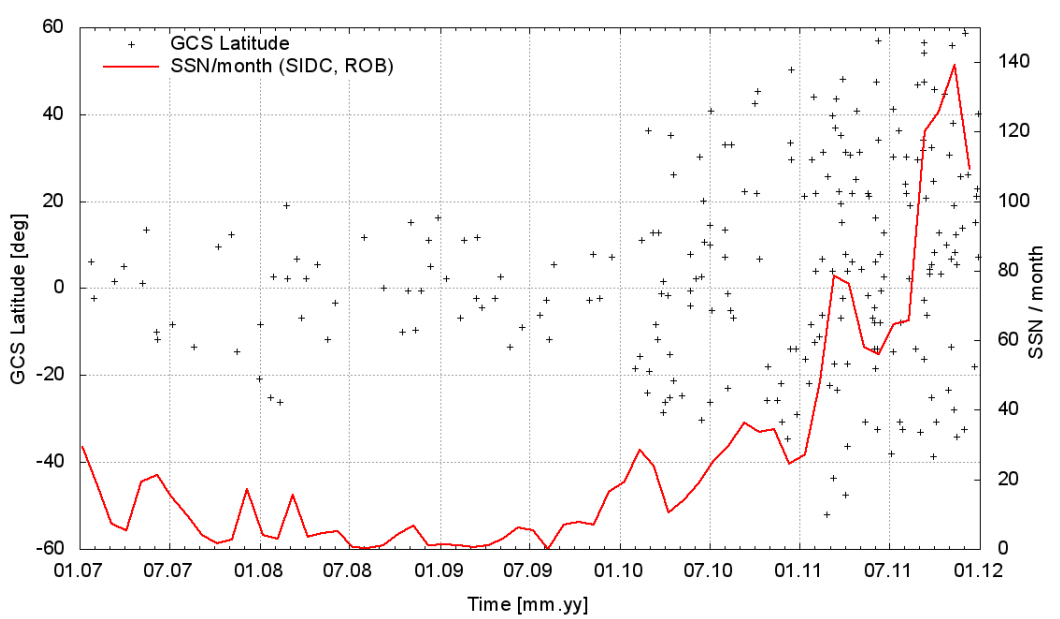

b)
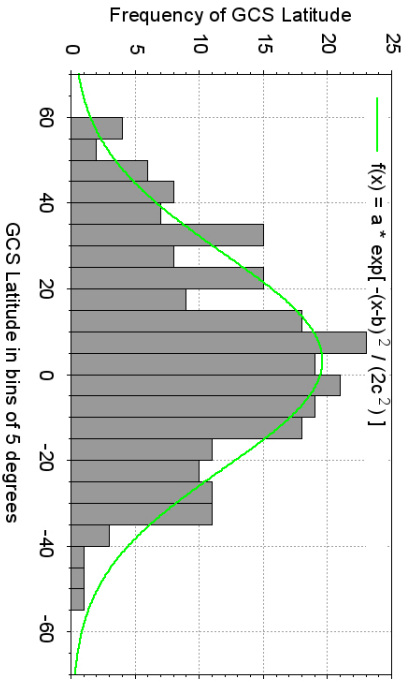

Fig. 63: Left: Latitude of the apex projection on solar surface of the modelled flux rope for 241 'Best-of' CMEs plotted vs. time; right: Frequency distribution of the GCS latitude with the median located at $3.07^{\circ}$. A fit of a Gaussian distribution is shown in green.

The frequency distribution with a median located at $3^{\circ}$ is shown on the right in Figure 63 in addition to the latitude distribution vs. time.

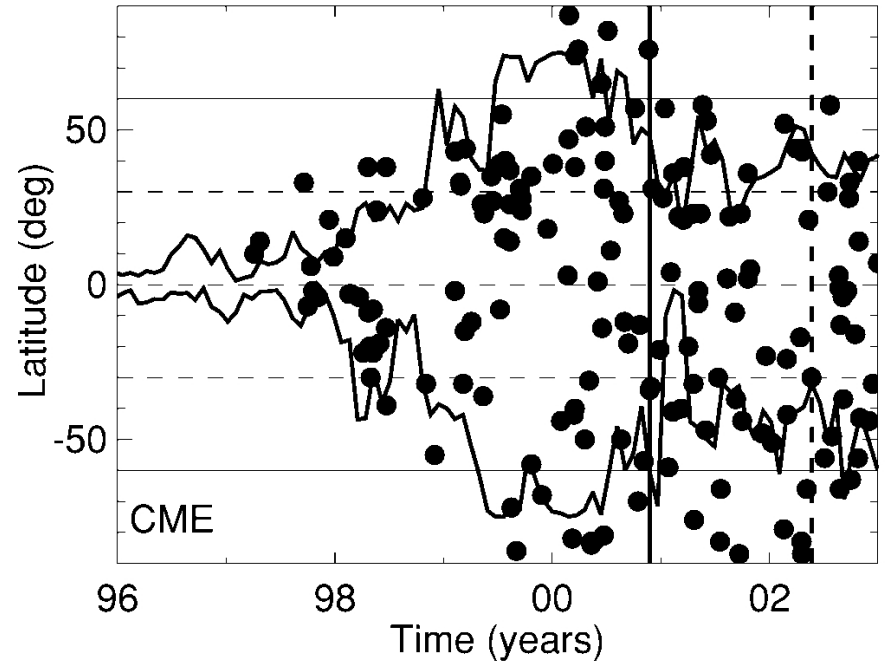

Fig. 64: The latitude of CMEs observed with SOHO/LASCO between 1997 and 2003 (Gopalswamy et al., 2003). The solid curves show the maximal deflection of the heliospheric current sheet. The vertical dashed and solid lines indicate the time of ending CME activity at high latitudes for the southern and the northern hemisphere, respectively.

For most of all CMEs (90\%) the latitude ranges between $\pm 40^{\circ}$. The latitude distribution of occurring CMEs within this time period can be described with a Gaussian distribution function. The fit yields 19 events for the functions maximum at $3^{\circ}$ latitude and for $\sigma=25$.

In order to judge the GCS latitude results better the synoptic magnetogram ${ }^{30}$ is shown in Figure 65 as an overlay to the GCS latitude datapoints. There blue and yellow indicate the polarity and intensity of photospheric magnetic regions, ranging from -10 G (blue) to $+10 \mathrm{G}$ (yellow). During solar minimum CMEs occurred at lower latitudes also when no strong magnetic field was observed at these regions. For a few CMEs in 2007 and 2008 the bipolar magnetic regions match very well to their latitude position. From 2010 solar activity increases indicated by strong magnetic regions at higher latitudes up to $50^{\circ}$ (southern) or $60^{\circ}$ (northern hemisphere), respectively. The occurrence

\footnotetext{
${ }^{30}$ The magnetogram is taken from Hathaway (2010), http://solarscience.msfc.nasa.gov/dynamo.shtml.
} 


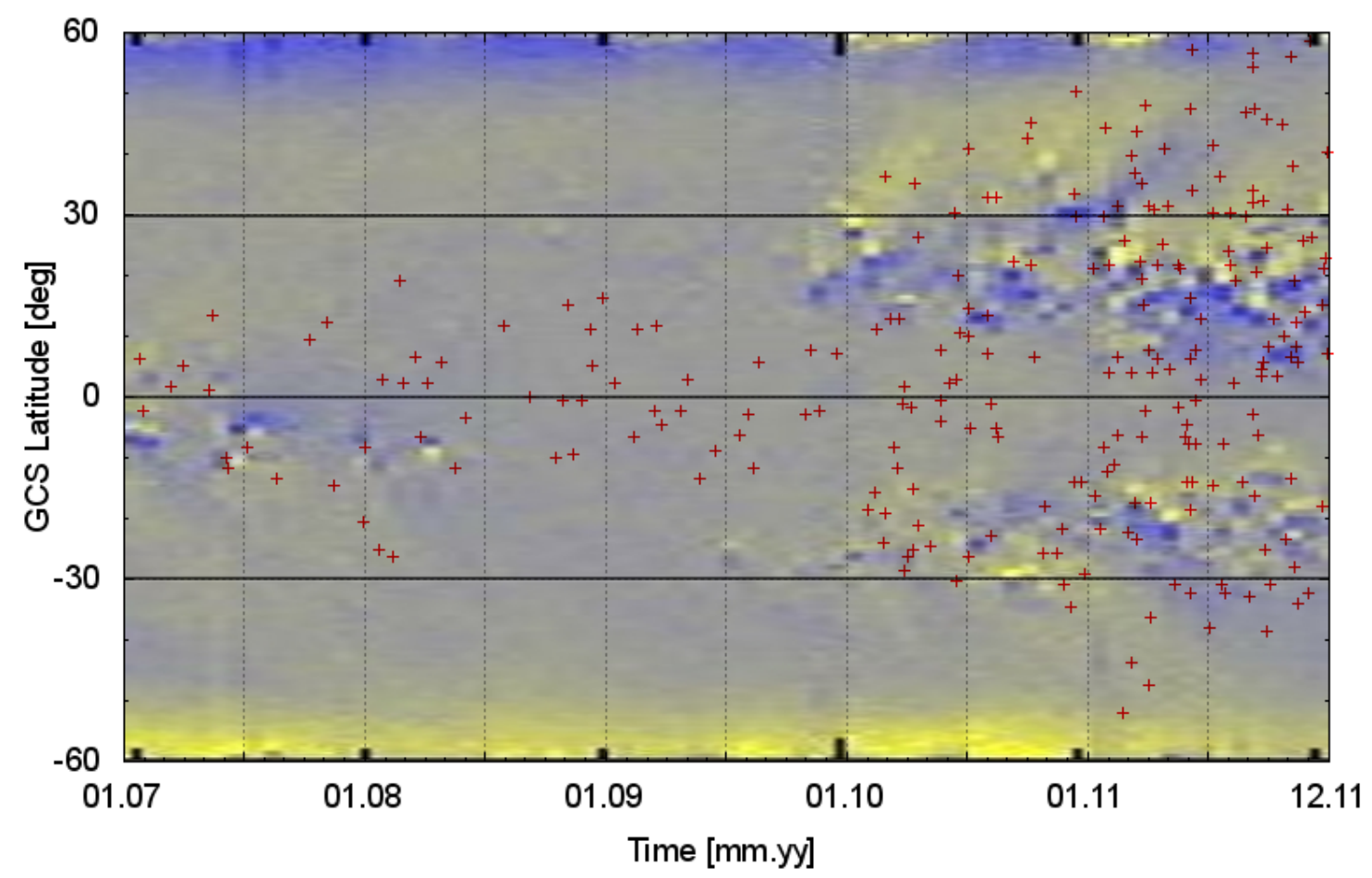

Fig. 65: The CME's apex latitude plotted vs. time with an overlay of a synoptic magnetogram from Hathaway/NASA/MSFC. Blue and yellow indicate the photospheric magnetic polarity and intensity ranging from $-10 \mathrm{G}$ (blue) to $+10 \mathrm{G}$ (yellow).

of CMEs at higher latitudes at this time matches very well with the magnetogram pattern. However also at this time several CMEs were observed at lower latitudes $\left[-15^{\circ}, 15^{\circ}\right]$ and near the solar equator where no strong magnetic regions are located. This could be an indication for CMEs which were originated at higher latitudes and deflected towards the solar equator as already published by Cremades and Bothmer (2004).

The presented correlations between the CME's latitude and the solar activity (i.e. CME latitude vs. time) are also already reported in former CME studies e.g. by Cremades and St. Cyr (2007). There it was revealed that the 'apparent' CME latitude remains at low latitudes during solar minimum and broadly spreads also to higher latitudes during solar maximum. This was observed for solar cycles 21 - 23 based on data of the MK3, Solwind, SMM and SOHO missions.

The crucial difference to this 3-D CME study is located in the determination of CME properties. In the pre-STEREO era it was only possible to calculate the parameters from plane-of-sky observations, like the latitude or the projected direction of propagation in longitude, respectively. With the usage of stereoscopic images from STEREO and applying the 3-D modeling technique to them the CME's properties can be determined without projection effects. This yields in more realistic and reliable results and finally confirms solid and in detail some of the previous study results, e.g. by Gopalswamy et al. $(2003,2006)$. However the presented detailed survey of CMEs with a 3-D modeling technique leads to a smaller data set of CMEs with 241 'Best-of' events in five years. This is owed to the time consuming modeling technique of CMEs. In contrast the mentioned statistical studies by Cremades and Gopalswamy comprise about 9000 CME events occurring over one complete solar cycle.

\subsubsection{Tilt Angle}

The next GCS parameter is the tilt angle which describes the inclination of the footpoint line of the flux tube relative to the solar equator. It ranges from $-90^{\circ}$ (perpendicular to the solar equator) 
over $0^{\circ}$ (parallel to the solar equator) to $+90^{\circ}$ (again perpendicular to the solar equator). The tilt angle of all 241 modelled 'Best-of' CMEs is plotted vs. time on the left in Figure 66. It is striking that the tilt angle in most cases ranges between $\pm 40^{\circ}$ for CMEs occurring between January 2007 and mid of 2010. After this time the tilt angle increases to higher values up to $\pm 80^{\circ}$ in several cases. The frequency distribution of the tilt angle is presented on the right with fit functions of a Gaussian distribution (green) and for comparison a cosine (blue). Both functions describe the frequency distribution well with the except of the three spikes at $-5^{\circ},-20^{\circ}$ and $-40^{\circ}$. The median of the Gaussian distribution is located at $-4.7^{\circ}$. For most of all CMEs $(80 \%)$ the tilt angle ranges between $\pm 40^{\circ}$ and $\sigma$ constitutes to $24.5^{\circ}$. The distribution describes the probability what tilt angle

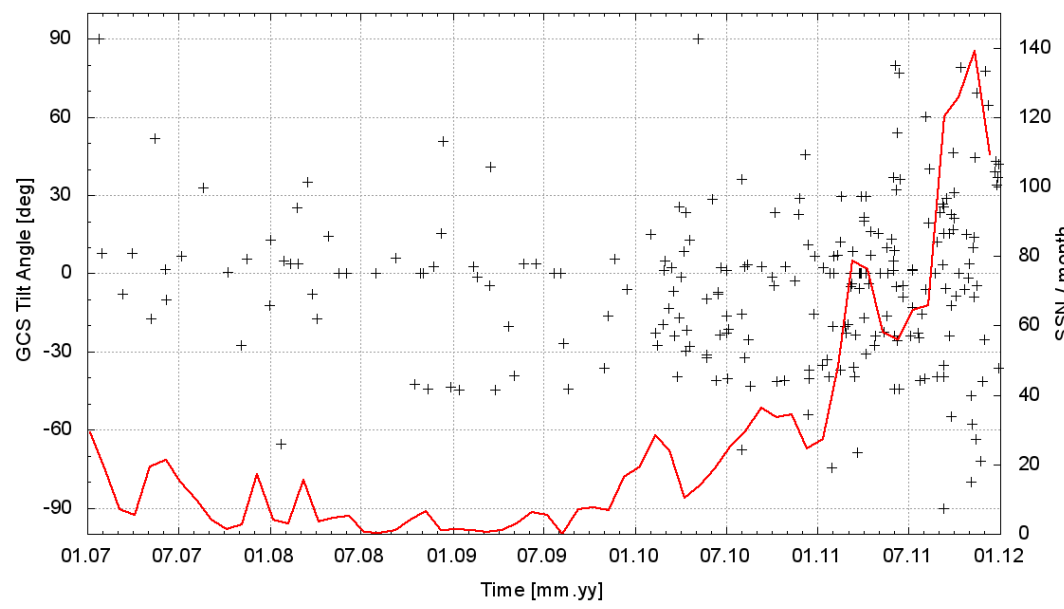

b) Number of events

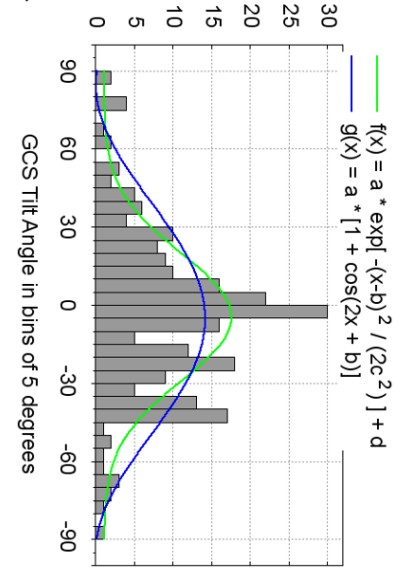

Fig. 66: Left: The GCS tilt angle (describing the inclination of the foot point line of the GCS flux tube relative to the solar equator) for 241 'Best-of' CMEs plotted vs. time. Right: Frequency distribution of the tilt angle with the fit function of a Gaussian distribution (green) and cosine (blue). The median of the Gauss function is located at $-4.7^{\circ}$.

value can be expected in general for a larger number of CMEs. Note that the expected typical tilt angle values differ for time periods with low and high solar activity, as shown in Figure 66 a).

\subsubsection{Aspect Ratio}

This GCS parameter is defined as $\kappa=a / r$ with the cross section radius $a$ of the tube section and the distance $r$ from solar center to the center of the cross section (see Figure 38 in Chapter 5.2). Hence this parameter stands for the constraint of self-similar expansion for CMEs as defined in the approach for the GCS model (see Section 5.2.1). The aspect ratio ranges from zero to one and is plotted vs. time in Figure 67 for all modelled 'Best-of' CMEs. Similar to the other parameters there is a different parameter behaviour in both time periods before and after February 2010. Before 2010 the aspect ratio varies between 0.2 and 0.4 in most cases whereas after that time the parameter spreads up to 0.8 for several CME modeling results. Besides the half angle, the aspect ratio is primarily responsible for the overall shape of the CME. That means that more smaller CMEs $(\kappa=[0.2,0.4])$ occurred in the first time period. In contrast more and more large-scaled CMEs $(\kappa=[0.2,0.8])$ were detected from February 2010 until December 2011. To get a better idea of flux-rope like CMEs with a 'smaller' or 'larger' aspect ratio an illustration is given in the appendix A.3. The frequency distribution of the aspect ratio is shown on the right of Figure 67 and is compared with a fitted Beta distribution function (green). For most of all CMEs (85\%) the aspect ratio ranges between 0.2 and 0.6, the maximum is located at 0.37. The Beta function was chosen for fitting because it is defined on a fixed interval similar to the aspect ratio which is defined between zero and one. 
a)

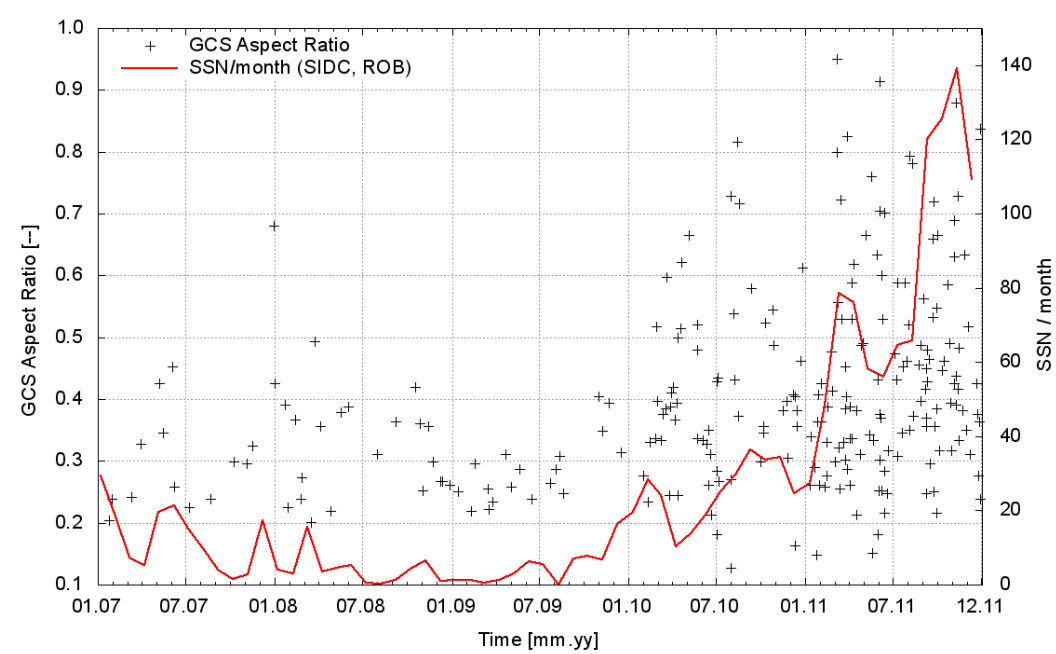

b)

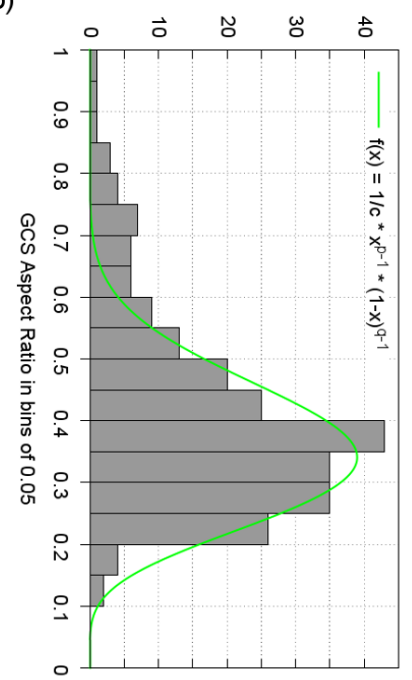

Fig. 67: Left: Aspect ratio of the GCS flux rope modelled for 241 'Best-Of' CMEs and plotted vs. time; right: Frequency distribution of the GCS aspect ratio with the median located at 0.37. A fitting function is given in green which compares the histogram with a Beta distribution.

\subsubsection{Half Angle}

The half angle $\alpha$ describes the angular expansion of the CME between both of its legs. The double value $2 \alpha$ defines the angle between the axes of both legs (see Figure 38 in Section 5.2) and is therefore not equal to the angular width because the angular width - applied on the GCS model - is defined as the angular difference between both outer boundaries of the legs. Based on the mathematical definitions of the GCS geometry by Thernisien et al. (2011) the angular width (AW) of a GCS-like CME can be calculated with:

$$
\begin{gathered}
\omega_{F O}=2(\alpha+\arcsin \kappa) \\
\omega_{E O}=2 \arcsin \kappa
\end{gathered}
$$

$\omega_{F O}$ and $\omega_{E O}$ denote the angular width of the GCS flux rope in the face-on or edge-on view, respectively. This is shown in Figure 69 b) and c). $\alpha$ and $\kappa$ are the same parameters as introduced for the GCS model in Chapter 5.2. The GCS half angle ranges from $0^{\circ}$ up to $90^{\circ}$ and can be considered as a dimension for its spatial extent. Note, that for $\alpha=0^{\circ}$ the axes of both legs overlie with each other and the GCS model turns into the cone model ${ }^{31}$. In this case the tilt angle becomes obsolete due to the rotation symmetry of the cone model. The half angle is also plotted vs. time in Figure 68 for 241 modelled Best-of CMEs. It is interesting to note that the half angle increases since February 2010 to larger values (here: $\geq 25^{\circ}$ ) whereas before this time almost all modelled GCS flux ropes exhibit smaller values. This is a behaviour similar to the aspect ratio parameter shown in the previous Figure which again indicates that since February 2010 more large-scaled GCS modelled CMEs occur. During the first time period (Jan. 2007 - Jan. 2010) the half angle ranges between $5^{\circ}$ and $30^{\circ}$ and later on rises up to $60^{\circ}$.

The frequency distribution for the half angle is shown on the right in Figure 68. The distribution can be well approximated with a Beta distribution function (green) which yields its maximum at $18^{\circ}$. For most of all CMEs $(78 \%)$ the half angle is smaller than or equal to $30^{\circ}$. The Beta function was chosen for fitting because it is defined on a fixed interval similar to the half angle which is defined between $0^{\circ}$ and $90^{\circ}$.

In previous CME surveys, e.g. by Cremades and Bothmer (2004) and Cremades et al. (2007), the

\footnotetext{
${ }^{31}$ The cone model was suggested by Zhao et al. (2002) and Xie et al. (2004) as an approach for a CME directed to the observer.
} 


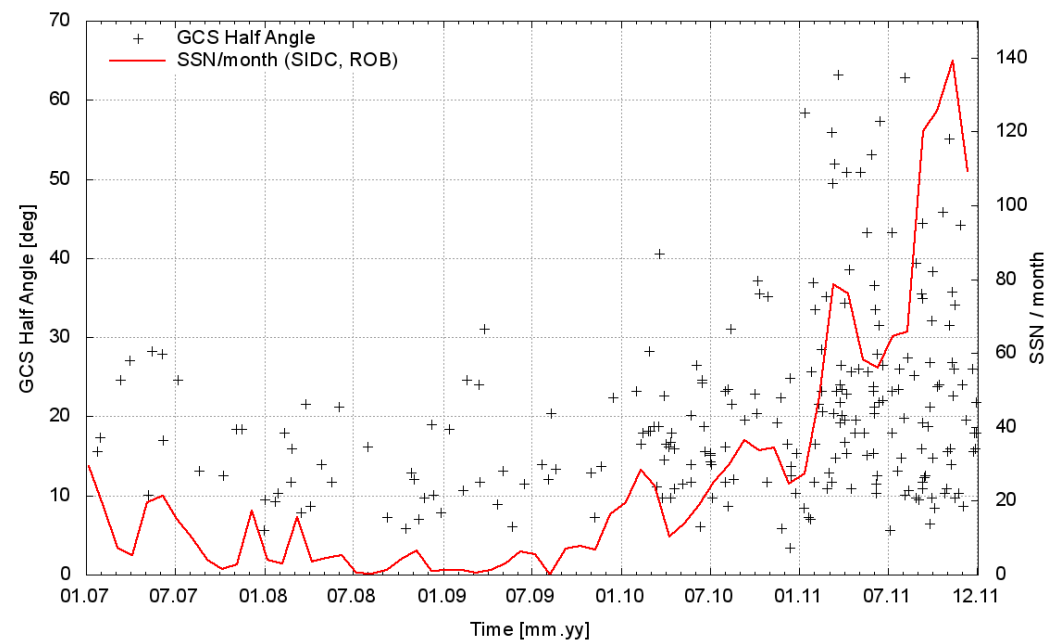

b) Number of events

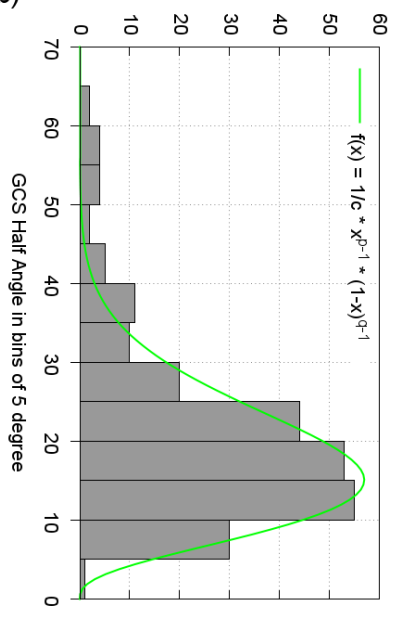

Fig. 68: Left: Half angle of the GCS flux rope modelled for 241 'Best-of' CMEs and plotted vs. time. Right: Frequency distribution of the GCS half angle with a fit (green) of a Beta distribution function yielding its maximum at $15^{\circ}$.

'apparent' angular width (AW) was inspected from plane-of-sky observations. The yearly average of the CME's angular width was set in relation with the solar activity (solar flux at $2800 \mathrm{MHz}$ ) and no significant correlation was found.

In order to give statements about the CME's angular width derived from the GCS half angle the latter was converted (see Equation $6 \& 7$ ) to the angular width seen in the face-on $\omega_{F O}$ and the edge-on view $\omega_{E O}$ (Figure $69 \mathrm{~b} \& \mathrm{c}$ ). Both angular widths describe the angular expansion for a GCS modelled CME in its 3-D position and orientation and therefore without projection effects related to the plane-of-sky. Figure 69 a) presents a diagram with both angular widths plotted vs. time. In general, the distribution of the angular width in edge-on view (green) is located at smaller

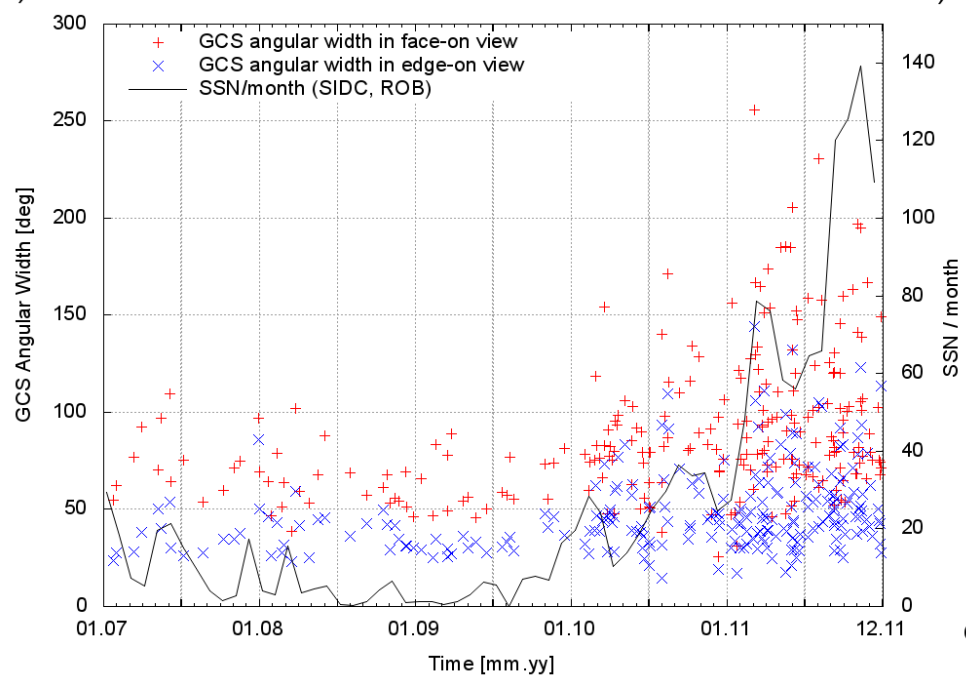

b) face-on view

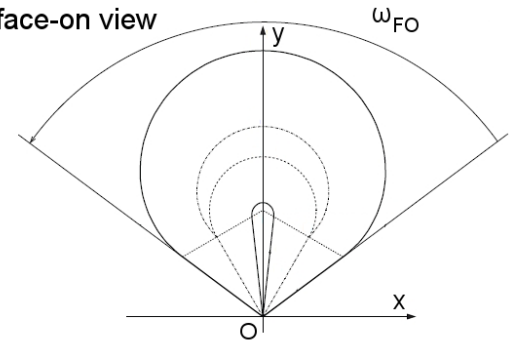

c) edge-on view

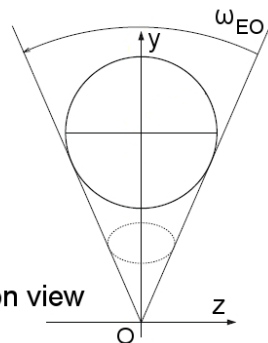

Fig. 69: The GCS angular width as seen in face-on (red) and edge-on view (blue) plotted vs. time. On the right is given an illustration of the angular widths $\omega_{F O}$ in face-on view b) and $\omega_{E O}$ in edge-on view c). The last two Figures were taken from Thernisien et al. (2011). 
values than the angular width in face-on view (red). This is due to the relation of $\omega_{E O}<\omega_{F O}$ which is a property of the GCS model. The behaviour of the angular width in relation to the time is in general the same as shown for the GCS Half Angle $\alpha$ in Figure 68. This is a consequence of the relationship between the angular width and $\alpha$ as shown in formula 6 and 7. An increase of the angular width since 2010 when solar activity rises is clearly visible. In conjunction with previous CME surveys it should be noted that the 'apparent' angular width measured in plane-of-sky cannot be compared 1:1 to the angular width determined with the GCS modeling technique which exhibits no projection effect.

The frequency distribution of the 'apparent' angular width was examined for about 9700 CMEs in a statistical study by Gopalswamy (2006). A similar distribution as shown in Figure 68 b) was found for these CMEs which were observed during solar cycle 23. The average value for the 'apparent' angular width in Gopalswamy (2006) yields $\sim 46^{\circ}$ and the peaks of the Beta distributions for the GCS angular widths $\omega_{F O}$ and $\omega_{E O}$ result in $73^{\circ}$ and $39^{\circ}$, respectively. The GCS angular width in edge-on view $\omega_{E O}$ which describes the 'thickness' of the tube section correlates directly to the flux rope's diameter which is discussed in paragraph 7.2.7.

\subsubsection{Height}

The height parameter $\mathrm{h}_{\text {front }}$ describes the distance from the center of the Sun to the apex of the CME's leading edge (see Section 5.2). The height parameter plotted vs. time is presented for all 241 'Best-of' CMEs in Figure 70. This diagram is characterised by a lower boundary for a height at $10 \mathrm{r}_{\odot}$ below which almost no modelled CMEs occur. Most of the events $(71 \%)$ spread between 10 to $16 \mathrm{r}_{\odot}$. This behaviour constitutes a subjective bias of the modeller's choice for the fitting images. The images given at a certain time-stamp were chosen depending on when the CME was best seen for modeling in the COR2 field of view $\left(2-15 \mathrm{r}_{\odot}\right)$. CMEs with a 'solar center - leading edge distance' lower than $10 \mathrm{r}_{\odot}$ are in general not enough expanded in shape and therefore not suitable for a good fit. In contrast the height parameter for a CME fit can achieve values
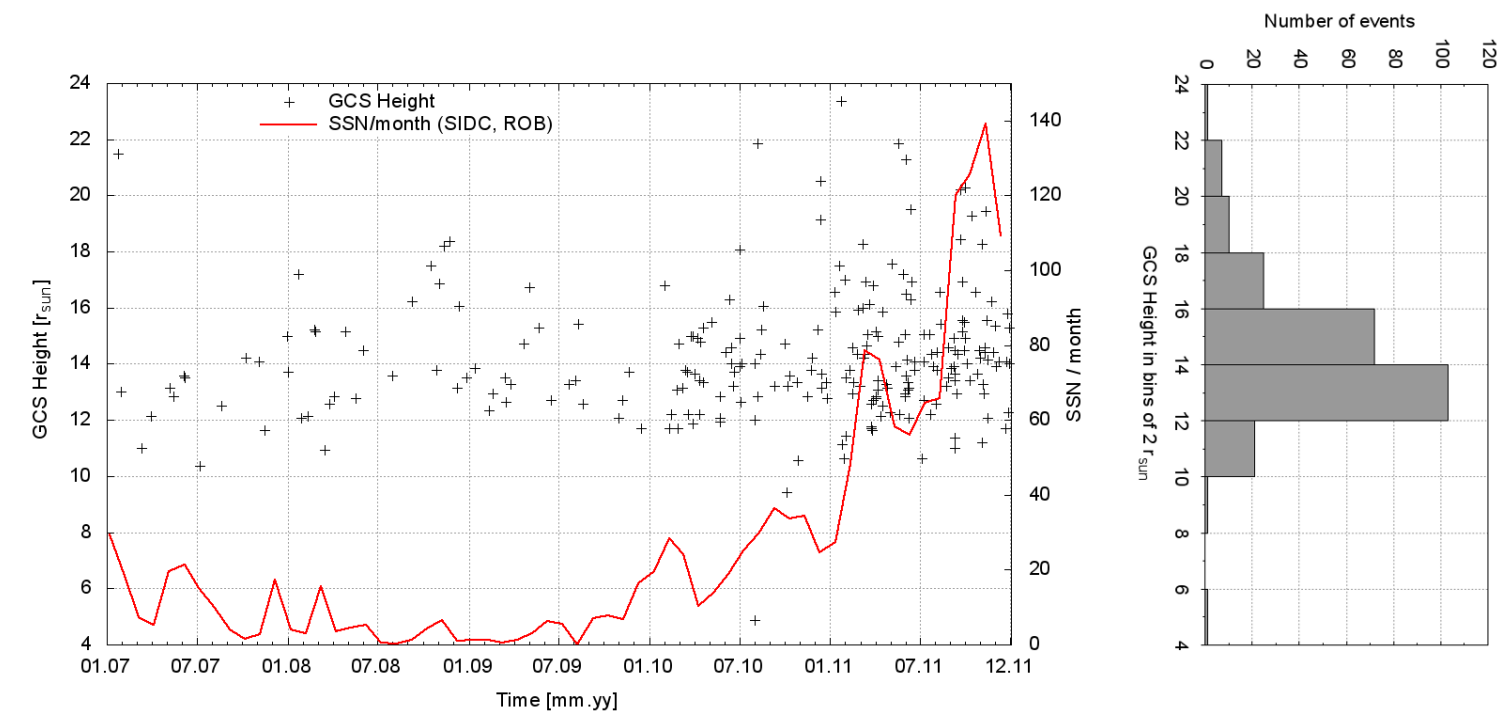

Fig. 70: Left: GCS height from solar center to the apex of the CME's leading edge modelled for 241 'Best-of' CMEs and plotted vs. time. Right: Frequency distribution of the GCS height with the median located at $14 \mathrm{r}_{\odot}$.

larger than the COR2 field of view due to projection effects. This is the case when the CME is propagating outwards with a larger angular distance $\gamma$ to the plane of sky, see Figure 71. A CME is propagating outwards in the direction which is indicated by the arrow $s_{3}$ with an angle $\gamma$ to the plane of sky of the observer. This direction of propagation is derived from GCS calculations 
with the length of $s_{3}$ given by $h_{\text {front }}$. The projection of the CME's direction of propagation to the plane of sky is denoted with $s_{1}$. The length of $s_{3}$ becomes larger with increasing angle $\gamma$. This

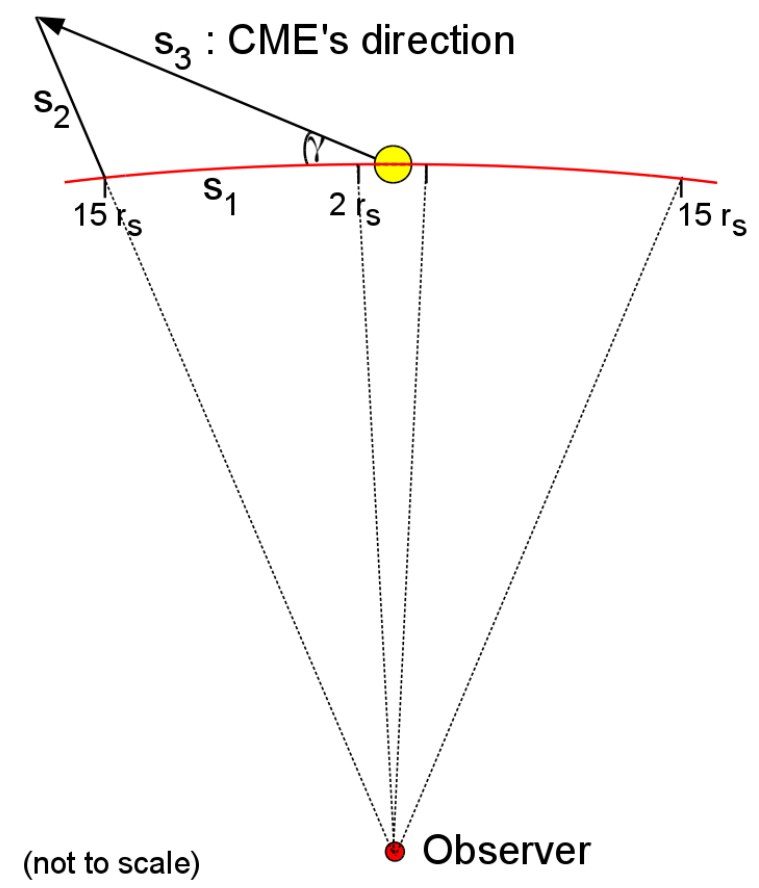

Fig. 71: A schematic illustration of a CME, which is propagating away from Sun. It's direction of propagation is indicated by the arrow $s_{3}$. The CME's direction is projected with the angle $\gamma$ to the plane of sky $\left(s_{1}\right)$ of the observer (red, bottom).

means that the CME's GCS height parameter can achieve values larger than the COR2 coronagraph's field of view. For this reason CMEs could be fitted at larger distances to the Sun, in some cases up to $23 \mathrm{r}_{\odot}$. At larger distances on the one hand the coronagraph's field of view limits the observation. On the other hand the CME's brightness and degree of clear recognisable structure decreases the ability for appropriate fitting. To sum up CMEs near the Sun are best suitable for fitting with the GCS model between 11 and $16 \mathrm{r}_{\odot}$ when the CME is sufficiently developed in shape.

\subsubsection{GCS Flux Rope Diameter}

The last CME parameter which is discussed in this section is the GCS flux rope diameter which designates the diameter of the cross section at the leading edge's apex. With that the diameter defines the elongation of the tube section at the CME's front in direction of propagation. The diameter $d=2 a$ is shown in Figure 72 on the left. It can be derived from the primary GCS parameters $\kappa$ and $\mathrm{h}_{\text {front }}$ given by the equation in the same Figure on the right. As introduced in Chapter $5.2 \mathrm{~h}_{\text {front }}$ names the distance from solar center to the apex of the CME's leading edge and $\kappa=a / r$ denotes the aspect ratio, see also Thernisien (2011). The diameter $\mathrm{d}$ depends on the aspect ratio $\kappa$ which reflects the model assumption of self-similar expansion for a CME. Therefore the diameter for an arbitrary chosen CME increases linear with rising height.

The introduction of the diameter parameter raises the question how large are CMEs expanded near the Sun in terms of their flux rope diameter? What diameters are typical for a CME at solar distances between $10-20 \mathrm{r}_{\odot}$ ? To answer these questions the flux rope's diameter was calculated for all 'Best-of' CMEs and plotted vs. the flux rope's height as shown in Figure 73. This diagram reveals which typical values for the diameter can be expected for a modelled flux rope at a given height (red data points). For instance CMEs observed at solar distances of $14 \mathrm{r}_{\odot}$ exhibit a flux rope diameter between 2 and $5 \mathrm{r}_{\odot}$. The diameter spreads to a larger range for CMEs at solar 

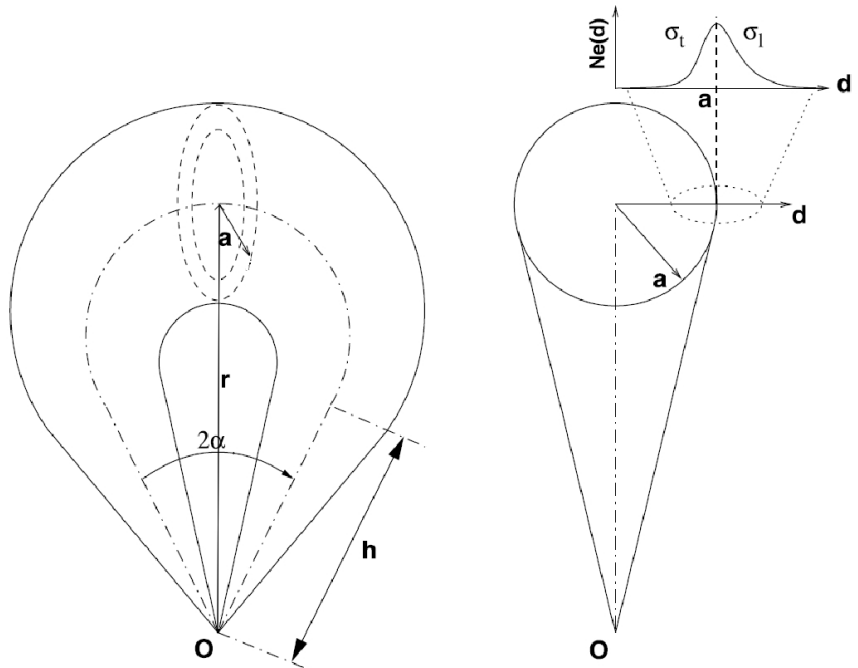

$$
d=\frac{\kappa \cdot h_{\text {front }}(1-\kappa)}{1-\kappa^{2}}
$$

Fig. 72: Left: The GCS flux rope in faceon and edge-on view. Right: The equation for the GCS flux rope diameter $d=2 a$ (Thernisien, 2011).

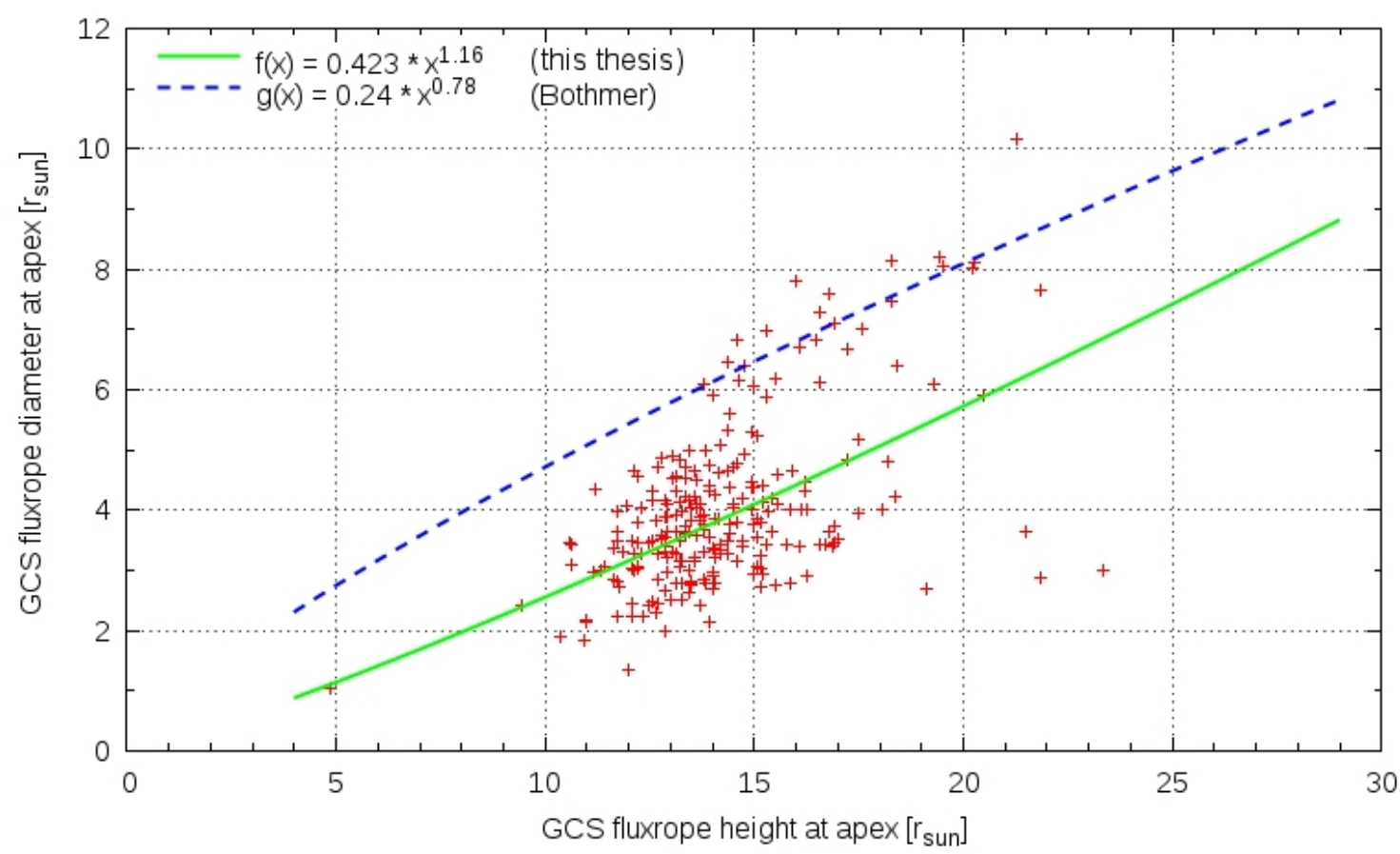

Fig. 73: The GCS flux rope diameter at the apex of the CME's leading edge plotted vs. the leading edge's distance to solar center (red). In green is shown a polynomial fit with the function given in equation 10. For comparison is shown the relationship between the magnetic cloud's diameter and their distance to Sun (blue) based on magnetic cloud observations with Helios data, published by Bothmer and Schwenn (1997). This function originally derived from magnetic cloud observations at $0.3-4.2 \mathrm{AU}$ is extrapolated for this plot to smaller solar distances.

distances larger than $15 \mathrm{r}_{\odot}$. More distant CMEs at $21 \mathrm{r}_{\odot}$ exhibit flux rope diameters between 3 and $10 \mathrm{r}_{\odot}$. A polynomial fit which indicates to first order their two-way dependence is presented in green.

The diameter or the cross-section area, respectively constitutes an important quantity for describing CME flux ropes as magnetic clouds with their magnetic field topology as introduced by Bothmer and Schwenn (1997). Within their study they described "magnetic clouds as large-scale cylindrical 
magnetic flux tubes". For magnetic clouds observed between 0.3 and 4.2 AU they found that the radial diameter $\mathrm{s}$ of the tube section is increasing proportional to the solar distance $\mathrm{R}$ with

$$
s(R)=0.24 \times R^{0.78}
$$

with $\mathrm{s}$ and $\mathrm{R}$ in $\mathrm{AU}$. By comparison, the dependence between the flux rope diameter $\mathrm{d}$ and the flux rope's solar distance $\mathrm{h}_{\text {front }}$ can be described with the fit function from equation 10 :

$$
d\left(h_{\text {front }}\right)=0.423 \cdot x^{1.16}
$$

This function is derived from the results of the GCS modeling for CMEs observed between 10 and $20 \mathrm{r}_{\odot}(0.05$ and $0.1 \mathrm{AU})$ as shown in Figure 73 with a polynomial fit (green line). These results are compared with the findings from Bothmer and Schwenn concerning the magnetic cloud's diameter for solar distances between 0.3 and 4.2 AU. Therefore the correlation from Bothmer and Schwenn is extrapolated to smaller solar distances (dashed blue line). Both functions show a different slope in the diagram. This is due to the different exponents which exhibit a value smaller 1 or larger 1 , respectively. The function from Bothmer and Schwenn confirms the diameter results comparably well for some CME events. But for most of the CMEs it overestimates the diameter roughly by $2 \mathrm{r}_{\odot}$.

\subsubsection{Summary}

The presented GCS analysis of the 'Best-of' CMEs revealed some major findings which are listed in the following summary.

- The STEREO CME list agrees very well with the SOHO LASCO CME catalogue for events with an angular width $\geq 45^{\circ}$.

- A dependence of the observed CME rate on the spacecraft's separation angle cannot be verified.

- The results of the GCS half angle, aspect ratio and latitude differ significantly between the two periods of time 01/2007 - 01/2010 (low solar activity) and 02/2010 - 12/2011 (increasing solar activity). Their frequency distributions can be described with suitable distribution functions (Gauss-, Cosine- and Beta- fit function, respectively). The numerical results of the parameters latitude, aspect ratio and half angle are summarised in Table 12. Most of the

\begin{tabular}{|c|c|c|c|}
\hline $\begin{array}{l}\text { GCS Model } \\
\text { Parameter }\end{array}$ & $\begin{array}{c}\text { Period 1 } \\
\text { (Jan/07 - Jan/10) } \\
\text { \% in Range }\end{array}$ & $\begin{array}{c}\text { Period 2 } \\
\text { (Feb/10 - Dec/11) } \\
\% \text { in Range }\end{array}$ & Comment \\
\hline Latitude [deg] & $96 \%$ in $[-20,+20]$ & $\begin{array}{l}46 \% \text { in }[-20,+20] \\
54 \% \text { in rest }\end{array}$ & $\begin{array}{l}\text { CMEs at lower latitudes } \\
\text { CMEs at higher latitudes }\end{array}$ \\
\hline Aspect Ratio [-] & $87 \%$ in $[0.2,0.4]$ & $\begin{array}{l}49 \% \text { in }[0.2,0.4] \\
48 \%>0.4 \\
3 \% \text { rest }\end{array}$ & $\begin{array}{l}\text { small-scale CMEs } \\
\text { large-scale CMEs } \\
<0.2\end{array}$ \\
\hline Half Angle [deg] & $78 \%$ in $[0,20]$ & $\begin{array}{l}52 \% \text { in }[0,20] \\
48 \%>20\end{array}$ & $\begin{array}{l}\text { small-scale CMEs } \\
\text { large-scale CMEs }\end{array}$ \\
\hline
\end{tabular}
analysed CMEs (provided in \%) exhibit values in the given range.

Tab. 12: The numerical results of the GCS latitude, aspect ratio and half angle from GCS fitting for 241 'Best-of' CMEs.

- The parameters GCS half angle and aspect ratio indicate from modeling that during the first time period more smaller CMEs occurred. In contrast more large-scale flux rope like CMEs were observed in the second time period.

- GCS Height: The GCS modeling analysis revealed that CMEs are best suitable for COR2 GCS modeling at solar distances between 11 and $17 \mathrm{r}_{\odot}$. Within this range of solar distance the CMEs appear largely expanded and show a bright and clear structure. 
- GCS flux rope diameter: The analysis of the diameter for GCS modelled CMEs provides an overview which elongations at the leading edge's apex can be expected in direction of propagation. The analysis reveals that CMEs observed between 10 and $20 \mathrm{r}_{\odot}$ exhibit a flux rope diameter ranging from 2 to $8 \mathrm{r}_{\odot}$.

- The crucial difference of this 3-D CME study to previous CME surveys is located in the determination of CME properties. In the pre-STEREO era it was only possible to calculate the parameters from plane-of-sky observations, like the 'apparent' latitude or the projected direction of propagation in longitude. With the usage of stereoscopic images from STEREO and applying the 3-D modeling technique to them the CME's properties can be determined avoiding projection effects.

\subsection{GCS Modeling Results and CME Classes}

The CME classes were introduced in Chapter 4 and the different CME morphologies were investigated with regard to the GCS flux rope model (Chapter 6). In this context the numerical results from GCS modeling are examined in relation to the CME classes and the different CME morphologies which they represent. To that the following questions are interesting: In which manner do these classes reflect characteristic properties of CMEs? Do CMEs of a particular class exhibit typical GCS parameter ranges or values? This section is addressed to these questions.

\subsubsection{GCS Aspect Ratio of CME Classes}

The GCS parameter aspect ratio reflects the shape of a modelled CME together with the half angle. To get an idea of the aspect ratio's effect on the CME's shape GCS flux ropes with different aspect ratio are provided in Figure 83 in the Appendix A.3. The distribution of this parameter for each CME class is shown in Figure 74. For each class their population in absolute numbers and percentage relating to the sum of 241 'Best-of' CME events is given at the bottom. The diagram reveals for some CME classes a concentration in the aspect ratio distribution. With two exceptions Halo CMEs exhibit an aspect ratio only with values between 0.6 and 0.85 . This fact reflects the large expansion of Halo CMEs and their GCS fitting at large solar distances when their overall circular shape is well expanded in the COR2 coronagraph's FOV. Also the events from the 'partial Halo' class show this behaviour. In contrast CMEs from the classes 'Ring'- , 'Jet-like'and 'Laurel wreath' CME show a smaller aspect ratio between 0.1 and 0.4 in agreement with their smaller expansion visible in the COR2's plane of sky. For the largest amount of analysed CMEs categorised as 'classical' CMEs the aspect ratio spreads over a large band width $(0.15-0.75)$. 


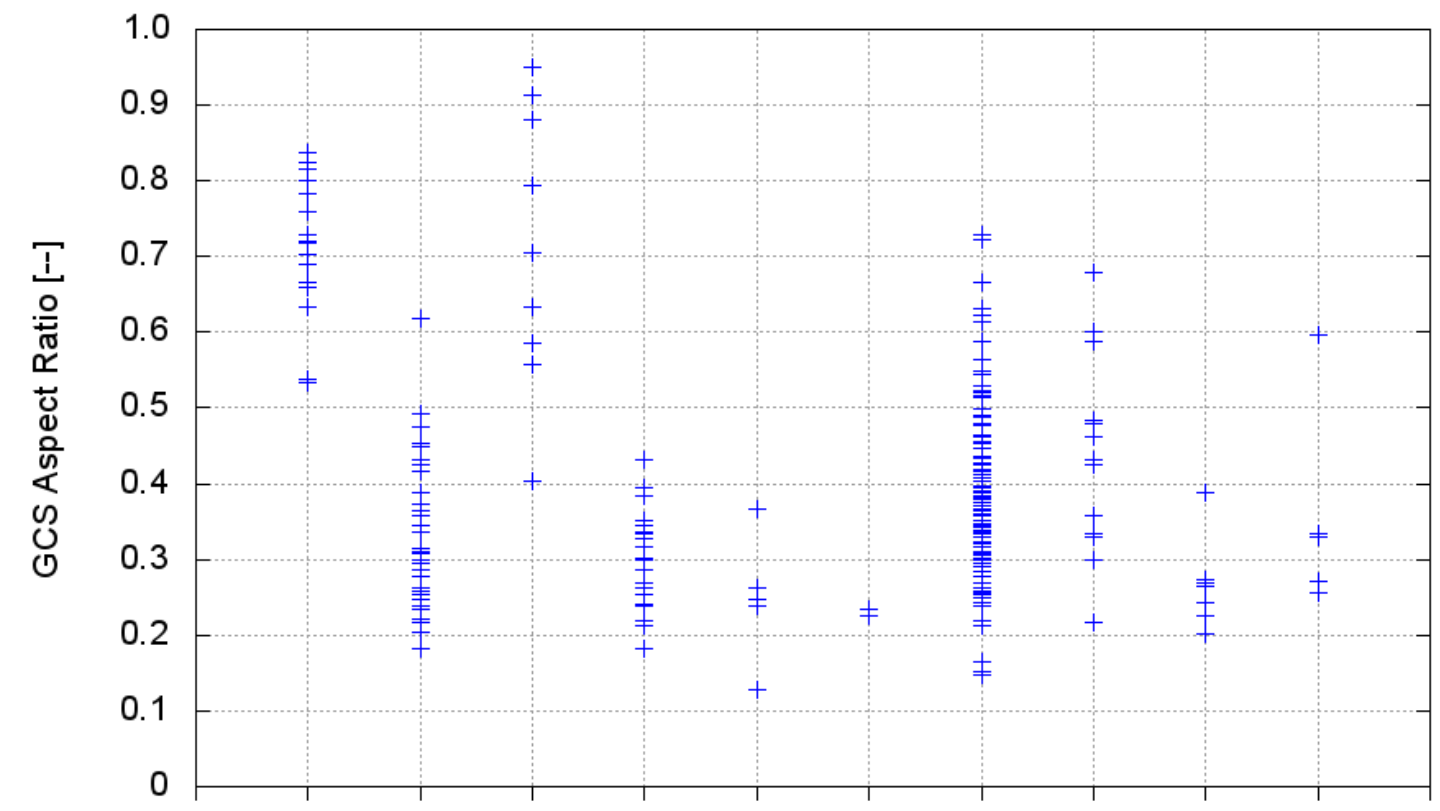

CME Class:

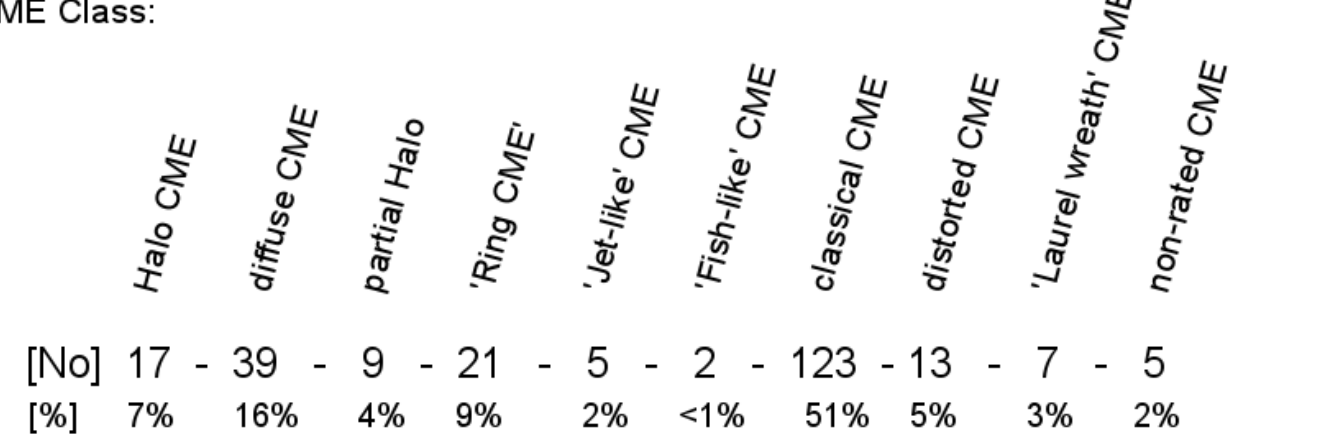

Fig. 74: The GCS parameter aspect ratio plotted for each CME in its related CME class. The amount of CMEs per class in absolute numbers and in percentage is given at the bottom.

\subsubsection{GCS Half Angle of CME Classes}

The half angle is the second parameter which describes the shape of a GCS modelled CME. Its distribution is presented for the CMEs of each class in Figure 75 in the same way as provided for the aspect ratio. It should be noted that for the large circular expanded Halo CMEs also small half angle ranging from $10^{\circ}$ to $20^{\circ}$ occur due to the "half angle - radial distance" ambiguity (as discussed in Section 5.4.8). The CMEs categorised as 'Jet-like'-, 'Fish'- and 'Laurel wreath' like CMEs and most of the 'Ring' CMEs show a typical small half angle $\left(5^{\circ}-25^{\circ}\right)$ which reflects their small angular width in the COR2's plane of sky. Again the 'classical' CMEs spread over a wide range of half angle values ranging from $5^{\circ}$ up to $60^{\circ}$ even though with an accumulation between $5^{\circ}$ and $30^{\circ}$ for most of them. 


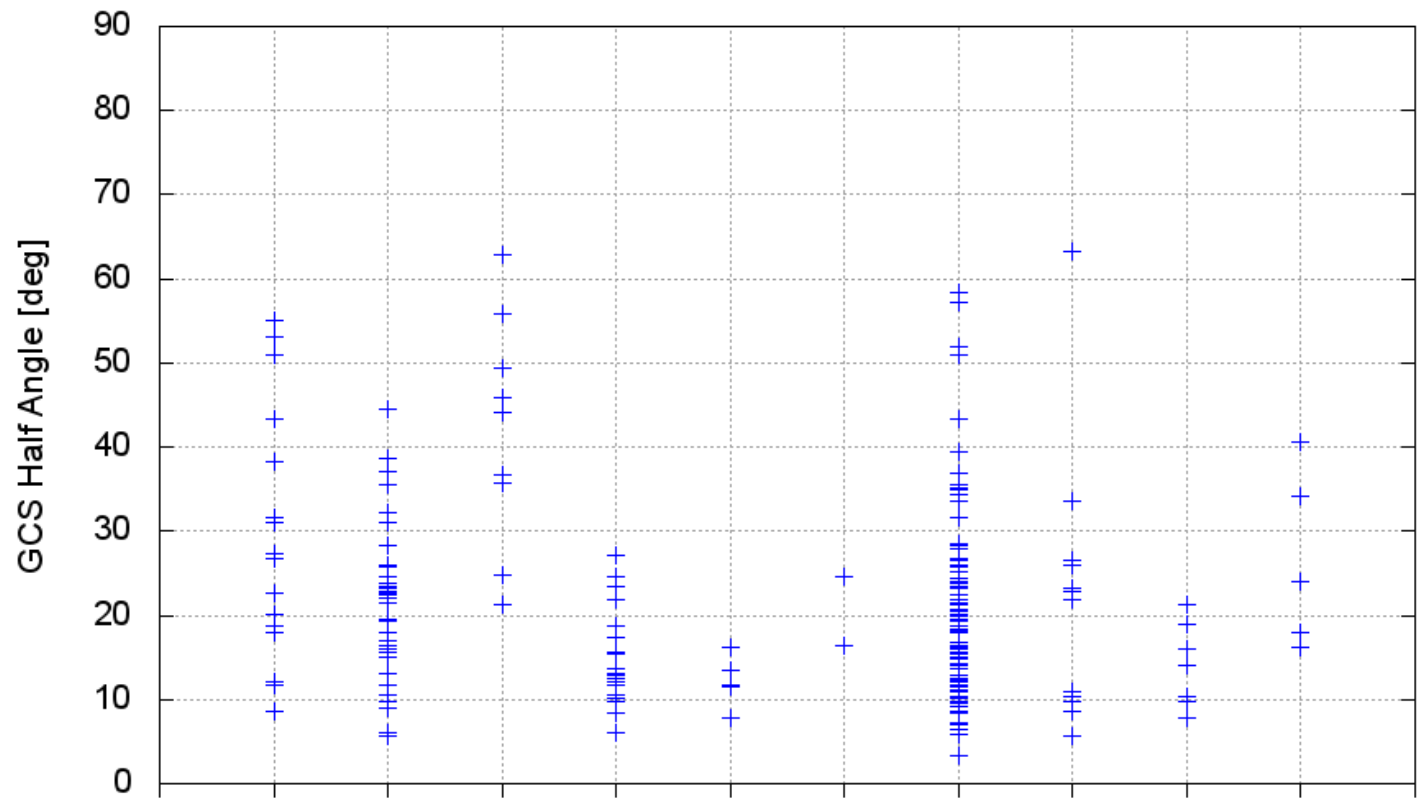

CME Class:

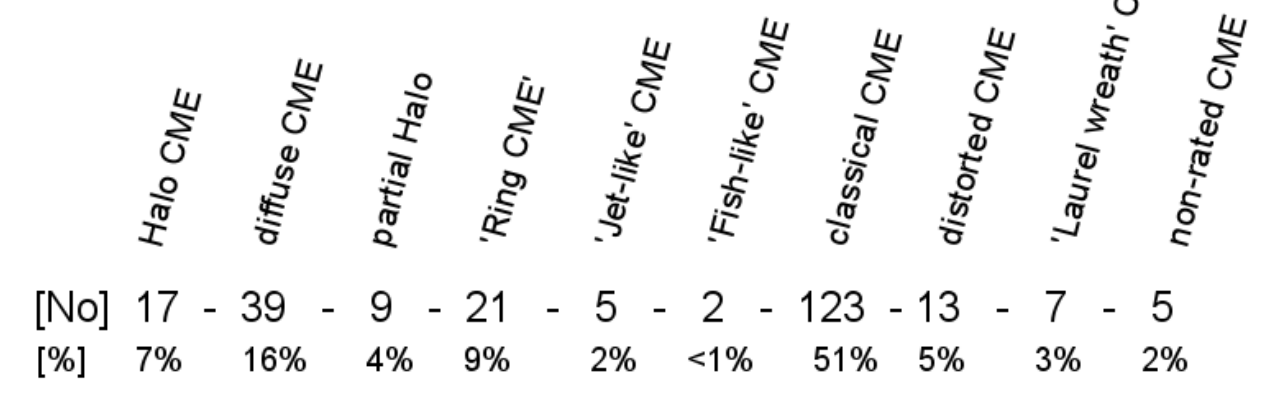

Fig. 75: The GCS half angle plotted for each CME of its class. The number of CMEs per class in absolute numbers and in percentage is given at the bottom.

\subsubsection{GCS Latitude of CME Classes}

The GCS latitude of the CMEs reveals some characteristics for their classes. As shown in Figure 76 the latitude of all Halo CMEs is concentrated within $-10^{\circ}$ to $+10^{\circ}$. This was expected for typical symmetrical Halo CMEs which are originated at the center of the solar disk. In contrast partial Halo CMEs were also originated at higher latitudes up to $45^{\circ}$ in both hemispheres. As shown before in Figures 63 and 65, the asymmetric distribution in latitude of CME events with a shift to the north is also distinguishable here. Especially, the CMEs of the classes 'diffuse CME', 'partial Halo CME' and 'classical CME' show this behaviour. As expected the 'classical' CME is found in large numbers at all latitudes up to $60^{\circ}$ in the northern and up to $-55^{\circ}$ in the southern hemisphere. CMEs with a distorted leading edge occur at a large range of latitudes, near the solar equator as well as at higher latitudes of $45^{\circ}$. CMEs small in angular expansion like the 'Fish', jetlike and 'Laurel-wreath' like CMEs occur with one exception only near the solar equator between $-15^{\circ}$ and $15^{\circ}$. 


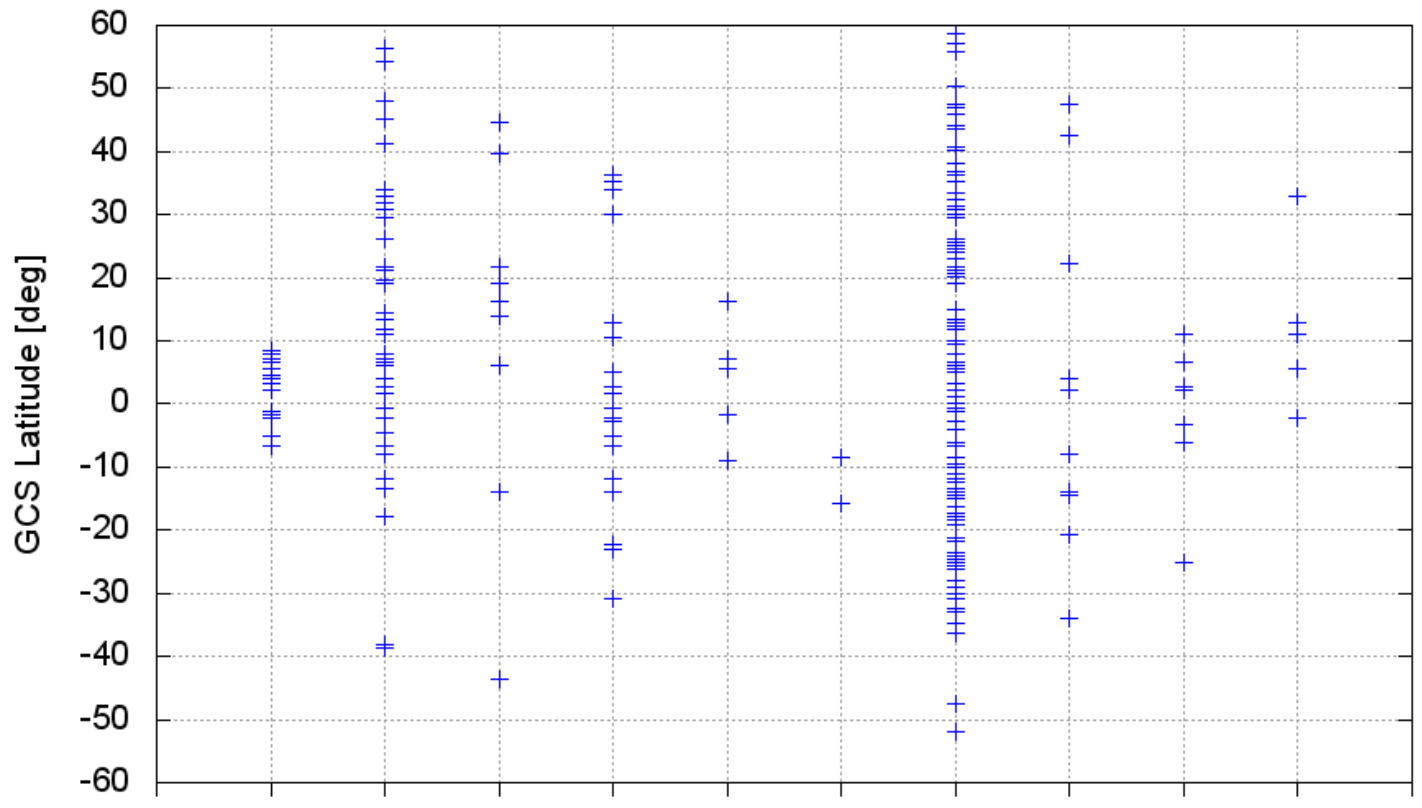

CME Class:

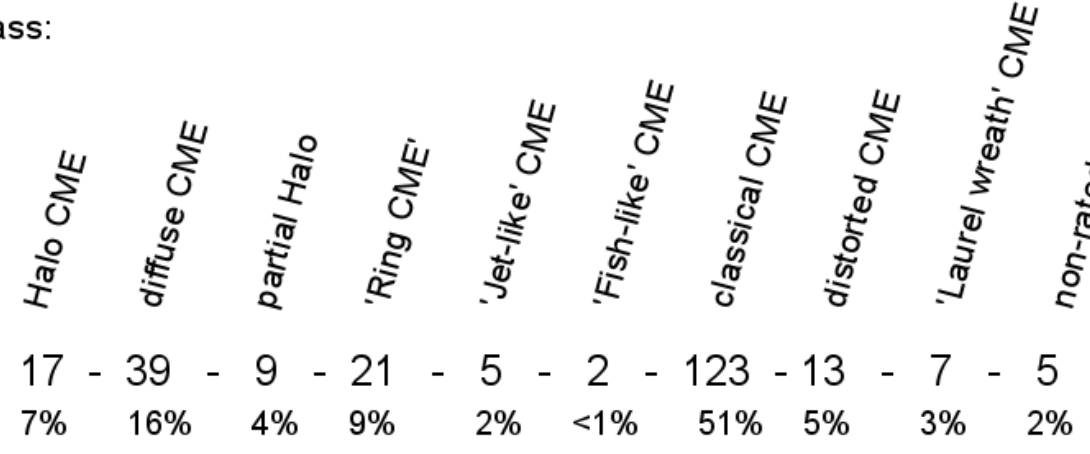

Fig. 76: The GCS parameter latitude plotted for each CME in its related CME class. The amount of CMEs per class in absolute numbers and in percentage is given at the bottom.

The remaining CME parameters and characteristics analysed with regard to the CME classes show no distinct features.

\subsubsection{Summary}

To summarise the presented results a brief overview is given below:

- GCS Aspect Ratio (CME's shape):

- Halo and partial Halo CMEs exhibit a large aspect ratio $(0.5$ - 1.0) due to their spatial expansion.

- CMEs with a small angular width (like 'Ring' CME', 'Jet-like' CME, 'Fish' CME and 'Laurel wreath' CME) show a small aspect ratio (0.1 - 0.45), also due to their small angular expansion.

- The aspect ratio for 'classical' CMEs spreads over a large range from 0.15 to 0.75 .

- GCS Half Angle (CME's shape):

- The 'Ring'- , 'Jet-like'- and 'Laurel wreath' like CMEs show a typical small half angle $\left(5^{\circ}-25^{\circ}\right)$ which reflects their small angular width. 
- The classical CMEs possess a half angle spreading over a wide range from $5^{\circ}$ up to $60^{\circ}$ even though with an accumulation between $5^{\circ}$ and $30^{\circ}$ for most of them.

- GCS Latitude (CME's position):

- As expected for solar disk centered Halo CMEs, their latitude is concentrated within $-10^{\circ}$ to $+10^{\circ}$.

- Partial Halo CMEs were also originated at higher latitudes up to $45^{\circ}$ in both hemispheres.

- As mentioned before (Figure 63, 65), the asymmetric distribution in latitude of CME events is also distinguishable with a shift to the northern hemisphere. This affects especially the CMEs classified as 'diffuse', 'partial Halo' and 'classical' CMEs which occur in large numbers.

- As expected, the 'classical' CMEs are found in large numbers at all latitudes up to $60^{\circ}$ in the northern and up to $55^{\circ}$ in the southern hemisphere.

\subsection{Applications for the GCS Modeling}

The analysis of CMEs with the GCS modeling technique does not only contribute to new scientific insights. The modeling of CMEs with the GCS technique improves methods in the field of space weather forecast, e.g. for assessing whether a CME hits Earth and with which velocity (Shi et al., 2015). In order to assess whether a CME hits Earth, it is necessary to determine as exactly as possible its direction of propagation in longitude and latitude. The calculation of the CME's half angle or angular expansion is required in order to estimate the 'cross section' of interaction between CME and Earth. This can help to answer whether the Earth would be strucked centrally or by a flank of the CME. The parameter results from GCS modeling (half angle, height) of a CME can improve the calculations of the CME arrival time at Earth with the Drag Force Model as discussed by (Shi et al., 2015). The CME's initial speed, as observed within the COR2 field of view, is important for the estimation of the CME's arrival speed and its arrival time at the Lagrangian point L1 in front of Earth. The initial speed for a CME can be derived from height-time plots based on calculations from several GCS fits for different points in time. CMEs measured near Earth are known as the major driver of temporal variations of the Earth's magnetic field. The largest disturbances of the geomagnetic activity are caused by CMEs which reach the Earth with a high velocity together with a negative magnetic field component $B_{z}$ (Bothmer and Daglis, 2007, Chapter 3.2.2 and 3.3.4).

In this day and age technical systems are affected by these temporal variations of the magnetic and electric fields in the magnetosphere and ionosphere. This concerns the disturbance of satellite communications, damage of electrical systems and enhanced radiation exposure for aviation and space flight. 



\section{Summary and Outlook}

This thesis reports on the 3-D analysis of coronal mass ejections (CMEs) in order to answer questions about their morphology and three-dimensional geometry. These are questions (Chapter 1.2) about the detailed 3-D geometry, orientation and position of CMEs which were observed near the Sun $\left(10-17 r_{\odot}\right)$.

The study for the 3-D analysis of CMEs started with the establishment of an overall CME list containing 1071 identified CMEs between January 2007 and December 2011 in observations from the STEREO/SECCHI/COR2 coronagraphs on-board the STEREO twin spacecraft. From this list a 'Best-of' CME list with 264 events was extracted, based on the visual appearance of the CME white-light structure in the coronagraph images. These CME events were analysed in this thesis in detail.

During the inspection of the COR2 coronagraph images it was noticeable that the CMEs occur with different morphologies in their two-dimensional white-light appearance. Ten CME classes were defined and introduced (Chapter 4) because several shapes of CME appearances with certain patterns occurred more often. Subsequently, the 'Best-of' CMEs were categorised according to these CME class definitions.

The different CME morphologies, which were found during the first inspections of the coronagraph data, were investigated in relation to the geometry of the flux rope model for CMEs. These examinations were addressed to the questions: Can CMEs of different white-light appearances be described as flux ropes with the Graduated Cylindrical Shell (GCS) model? And second, how does these CMEs look like in terms of this model? In order to clarify these questions a CME from each class was fitted with the GCS modeling technique to determine its 3-D geometry. In a second step synthetic coronagraph images were computed with a ray-tracing code for each CME. These were compared to the original coronagraph images. It was found that the different appearances and morphologies of CMEs observed with coronagraphs can indeed be described with the GCS flux rope model. Their different morphologies can be fitted with the GCS modeling technique resulting in a 3-D flux rope geometry. The synthetic coronagraph images, which were generated from Thomson scattering calculations, confirm their 3-D flux rope geometry (Chapter 7.3).

Based on these results the 3-D geometry, position and orientation of 241 'Best-of' CMEs was analysed and discussed in the second analysis part of this thesis (Chapter 7). For this purpose the GCS modeling technique was used to compute the CME's 3-D geometry with STEREO/SECCHI/COR2 coronagraph data. The difference of this study to previous ones is located in the detailed 3-D parametrisation and analysis of a large set of $241 \mathrm{CMEs}$ instead of plane-of-sky measurements which are affected with projection effects. The key results and the answers to the scientific questions introduced in Chapter 1.2 are presented in the following section.

A CME online database was developed within this work. It is available at www. affects-fp7.eu/ cme-database. This CME database comprises all mentioned lists of CMEs, the CME modeling results and additional graphical material (coronagraph images, source region images, fit images). This database is described in the Appendix A.6.

\subsection{Scientific Questions and Answers}

The results from the 3-D modeling of CMEs and their analysis within this thesis allows to answer the scientific questions from the beginning (Section 1.2). The answers are summarised in this section.

\section{To which extent do the observer positions of the STEREO spacecraft influence the 3-D GCS modeling of CMEs?}

The application of the 3-D GCS CME modeling is difficult and ambiguous for an angular spacecraft separation less than $10^{\circ}$. This is due to the low angular separation of both observer which results in very similar CME appearances in the coronagraph images. This was the case 
during the STEREO mission for the time period from January to May 2007. The same applies for the time period of the beginning in 2011 when both STEREO spacecraft were separated by $180^{\circ}$ (February 2011). In all other cases a 3-D GCS parametrisation of CMEs can unambiguously be performed because the angle between the plane of skies of both STEREO coronagraphs is large enough to resolve the CME in all dimensions. For further information, see also Section 5.4 .8 .

2. Which types of CMEs allow a description with the GCS model? What are the difficulties?

The analysis of different CME morphologies observed with the STEREO coronagraphs reveals that their white-light structure can be reproduced very well with the GCS model in agreement with the flux-rope model for CMEs.

CMEs with a distorted leading edge are difficult to fit. A distorted CME front cannot be fitted in an appropriate manner with a modeling technique based on a model which describes the CME's leading edge with a circular curved front (Section 5.4.4).

CMEs which show bright ambient shock material in their white-light appearance, especially in front of their leading edge, are also difficult to fit. In this case the ambient bright shock material can be misinterpreted as part of the CME and therefore falsify the CME's GCS parameters. Further details are presented in Section 5.4.5.

\section{How does the GCS model look like for a flux rope CME? What are typical 3-D} properties?

It has been shown in this thesis (Chapter 5.4 and 6) that CMEs can be described with the GCS flux rope model. Their three dimensional geometry calculated with the GCS modeling technique for 241 CMEs which occurred during 2007 and 2011 is presented on images in this thesis and in the online database at www.affects-fp7.eu/cme-database. It was found that the CME's GCS latitude is in most cases in agreement with bipolar magnetic regions (possible indicator for CME source regions) from a synoptic magnetogram. The GCS parameters half angle and aspect ratio reveal from modeling that only small-scaled CMEs occurred during the time period of low solar activity (Jan 2007 - Jan 2010). A half angle $\alpha \leq 20^{\circ}$ and an aspect ratio ranging from 0.2 to 0.4 is characteristically for those small CMEs. Whereas during the second time period with increasing solar activity (Feb 2010 - Dec 2011) also large scale CMEs are observed and CMEs occur also at higher latitudes up to $\pm 60^{\circ}$. These CMEs exhibit an angular expansion with a half angle $\alpha>20^{\circ}$ and an aspect ratio $\kappa>0.4$. In order to get an idea of GCS flux ropes with different aspect ratios some examples are shown in the Appendix A.3. The analysis of the diameter for GCS modelled CMEs reveals that CMEs observed between 10 and $20 r_{\odot}$ exhibit a flux rope diameter ranging from 2 to $8 r_{\odot}$. The observed CMEs are best suitable for GCS modeling within a solar distance of 11 to $17 r_{\odot}$ when they are largely expanded and show a bright and clear structure. All results are without projection effects in relation to the coronagraph's plane of sky. This is in contrast to previous larger CME surveys, e.g. by Gopalswamy et al. (2003, 2006).

4. The different classes of CME morphologies and their 3-D properties: Which characteristics in terms of GCS CME parameters does a CME classification reflect?

Different CME morphologies observed in the STEREO/SECCHI/COR2 coronagraph images were analysed with the GCS modeling technique. It was found that their different white-light appearances can be reproduced with the GCS model of a flux rope (Chapter 6). The synthetic coronagraph images which were generated from Thomson scattering calculations confirm their 3-D flux rope geometry. The analysis of the different CME class morphologies in conjunction with the results from the three dimensional GCS modeling suggests that the CMEs can be 
grouped in small-scale, large-scale and 'other' CMEs.

Small-scale CMEs like the 'Laurel wreath', 'Fish' and 'Ring' -type CME can be best described with the GCS model in edge-on view and a tilt angle nearby $0^{\circ}$. In this case the flux rope is oriented parallel to the solar equator.

The CMEs of the classes 'classical', '(partial) Halo' and 'diffuse' CME represent large-scale CME events. Most of their different shapes which are visible in the coronagraph images are most likely due to projection effects as supposed by Schwenn (2006).

'Jet-like' CMEs and those which exhibit a distorted leading edge are considered as 'other' CMEs. For the latter it was possible to reproduce their overall shape aside from their deformed front. The GCS modeling revealed some characteristics for the CMEs and their associated classes.

The latitudinal position of Halo CMEs is found as expected at low latitudes $\left(-10^{\circ}\right.$ to $\left.+10^{\circ}\right)$. In contrast partial Halo CMEs are located between $\pm 45^{\circ}$. And classical CMEs spread over latitudes between $-55^{\circ}$ and $+60^{\circ}$. The analysis of the CME's shape reveals that (partial) Halo CMEs exhibit large aspect ratios $(0.5-1)$ due to their spatial expansion. In contrast the aspect ratio for classical CMEs spreads over a large range $(0.15$ - 0.75) and their half angle spreads over $5^{\circ}$ to $60^{\circ}$. CMEs with a small angular width tend to a smaller aspect ratio $(0.1-0.45)$. This is the case for 'Ring', 'jet-like', 'Fish' and 'Laurel wreath' like CMEs.

For further details the reader is referred to Sections 6 and 7.3.

\section{What are the applications for 3-D GCS modeling of a CME?}

The 3-D parametrisation of a CME is used in the field of space weather forecast. The GCS longitude and latitude helps to assess whether a CME hits Earth or not. The CME's angular expansion can be derived from the GCS angular width (and aspect ratio). Hence the CME's cross-section for an interaction with Earth can be estimated. And this helps to judge whether the Earth would be strucked centrally or by the CME's flank. The CME's initial speed can be determined with height-time plots derived from multiple GCS fits. The initial speed is required (amongst other factors) for the calculation of the CME arrival time and arrival speed at Earth or L1, respectively. Further information can be found in Section 7.4.

\subsection{Outlook}

Within the scope of this work further questions occurred and later on improved fitting methods were published. Also new approaches for enhanced CME models were discussed. The following issues constitute very interesting research projects for the future.

The source region of a CME can provide hints to CME properties like its geometry or orientation. Cremades and Bothmer (2004) found strong correlations between the characteristics of photospheric source regions and the associated CMEs. A question for further studies is, to what extent does the CME's geometry depend on the CME's associated photospheric source region? The source region's expansion in longitude and latitude could depict an influencing factor to the CME expansion. The distance between the GCS foot points and therefore the GCS angular width in face-on (and edge-on) view could depend on these source region characteristics. The dependence between the tilt angle of source region's neutral line and the CME orientation (GCS tilt angle) can be analysed as suggested by Cremades and Bothmer (2004).

The examination of the magnetic field structure of the CME's source region constitutes another challenge for upcoming research topics. To what extent does the source region's magnetic field structure and intensity influence the CME properties? What are the source region differences for small and large-scale CMEs (e.g. in terms of the GCS half angle, aspect ratio)? The magnetic structure of the source region can be compared in detail to the GCS flux rope position and especially to the position of the foot points. These investigations can help to enhance the geometric GCS model with a magnetic field structure as suggested by Cremades and Bothmer (2004). The cause of a distorted leading edge of CMEs is not yet clarified. And CMEs with a non circular 
front cannot be analysed with the present Graduated Cylindrical Shell model. How can the GCS model be improved to describe CMEs with a non-circular leading edge? In past discussions with scientists some ideas came up for a detailed mathematical approach with trigonometric functions for a 3-D model which allows to adjust a non circular curved front to distorted CMEs. This approach provides the possibility to enhance the Graduated Cylindrical Shell model for sophisticated analyses of more complex CME geometries. 


\section{A Appendix}

\section{A.1 The IDL Code for Data Processing}

The IDL routines which are used for processing the FITS data are presented in this section. The procedure for modeling a CME with the GCS IDL routines is described in chapter 5.3.3.

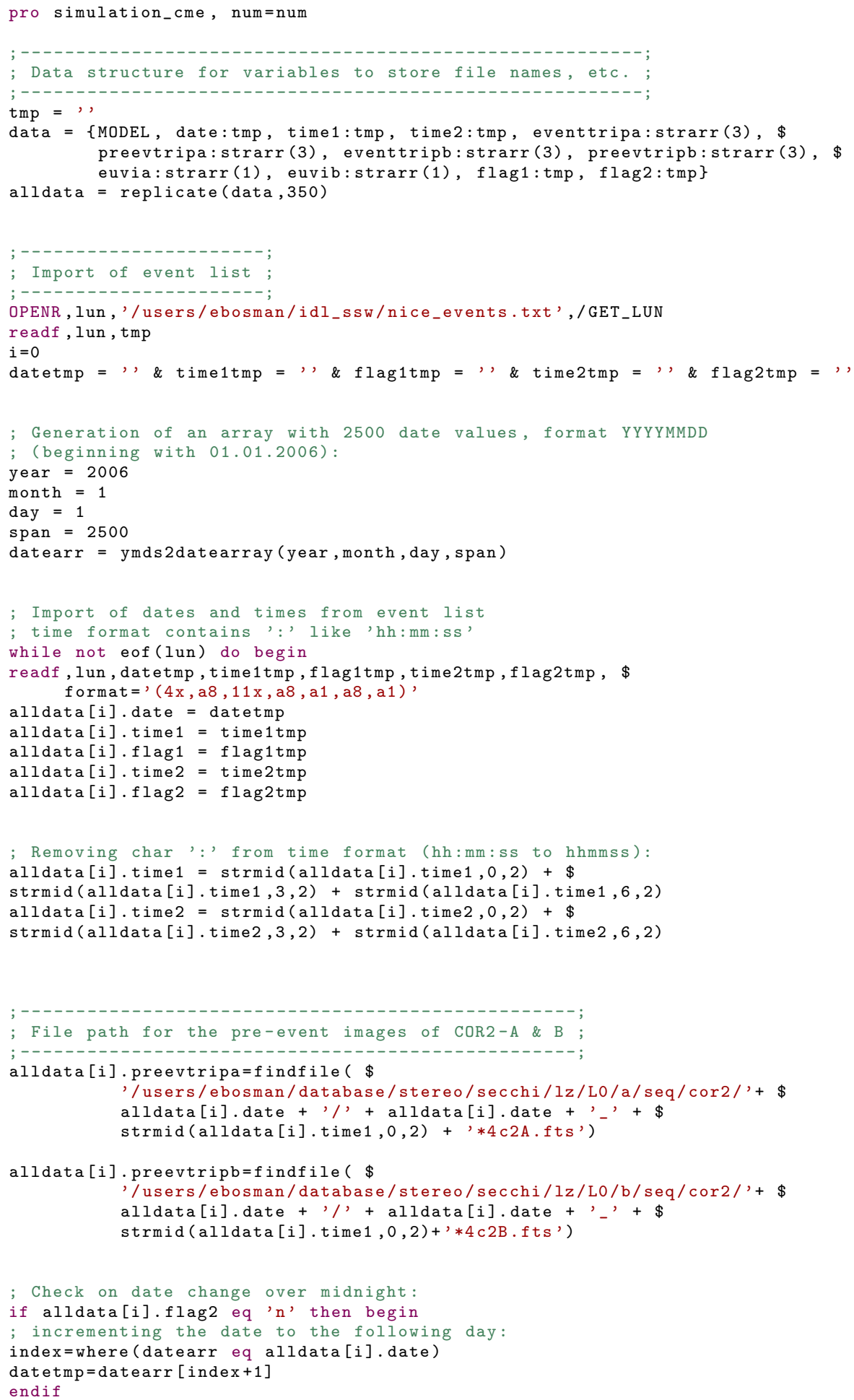




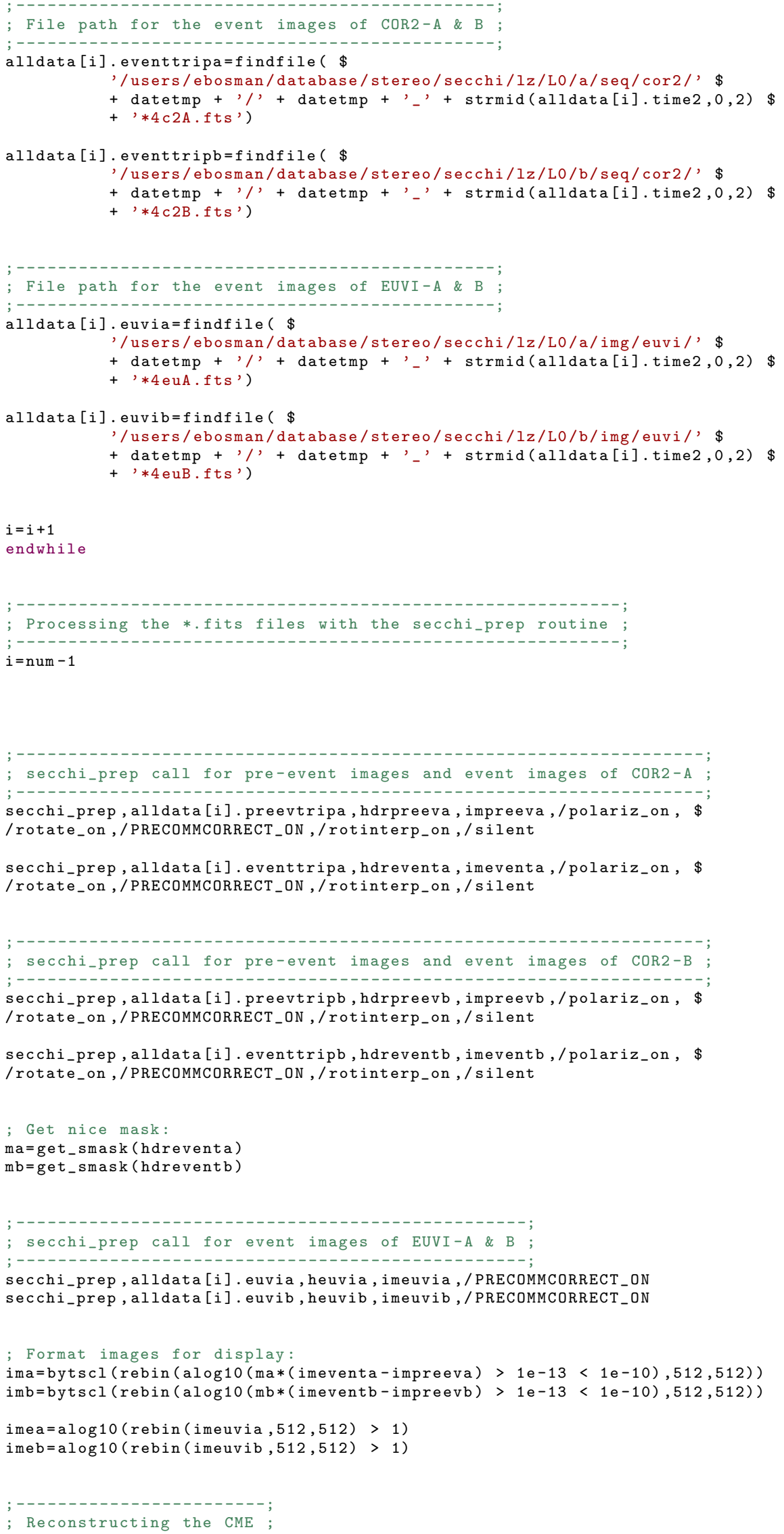




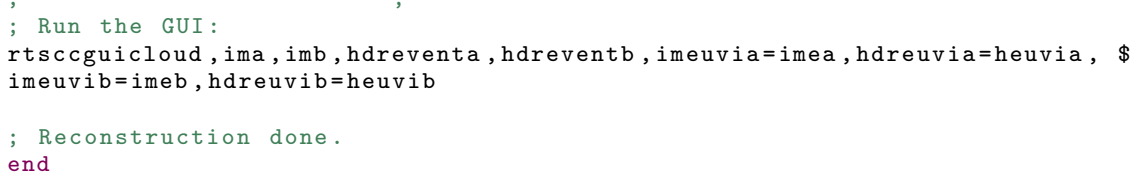

\section{A.2 Selected CME Classes and the Flux Rope Model}

The CME classes and their analyses with synthetic coronagraph images was discussed in Section 7.3 for four classes. The remaining CME classes, each with a CME shown in white-light and with the associated synthetic coronagraph images, are provided in this section.

\section{A.2.1 CME Class 02 - Diffuse CME}
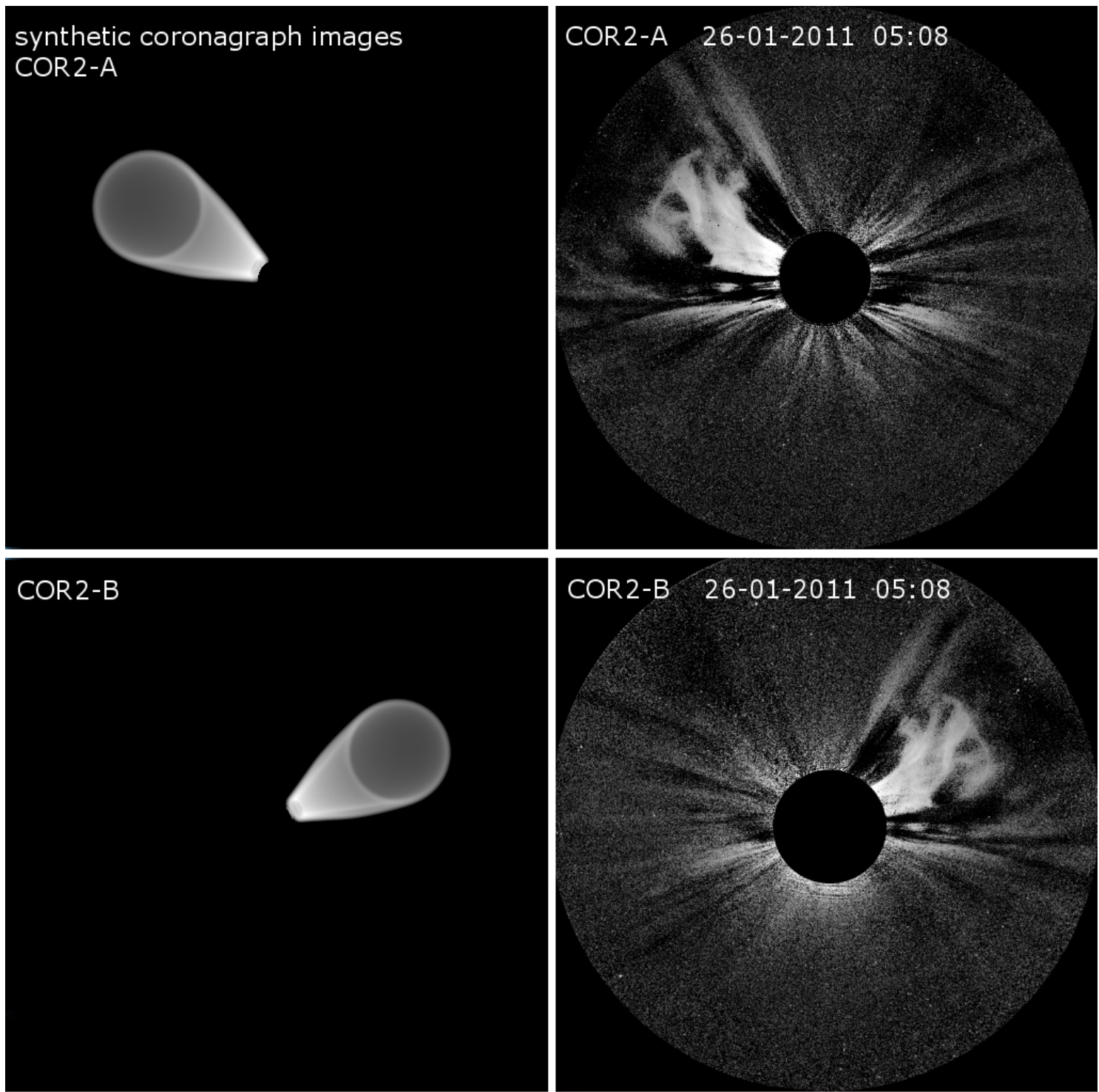

Fig. 77: Right: A CME classified as 'diffuse CME' observed on January 1st, 2011 by STEREO/SECCHI/COR2-A and -B. Left: The corresponding synthetic coronagraph images from calculations with the GCS modeling technique. 


\section{A.2.2 CME Class 03 - Partial Halo CME}
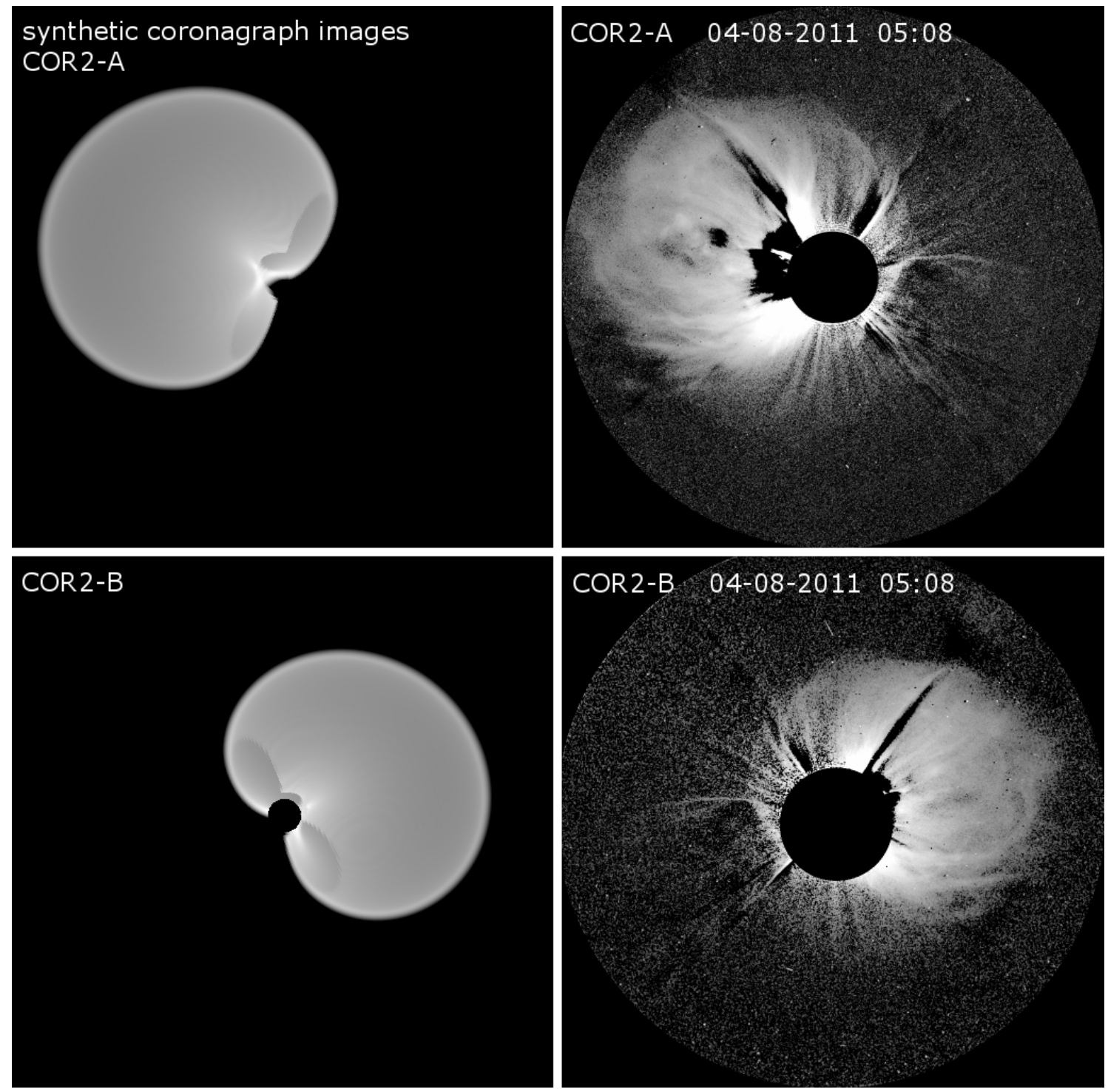

Fig. 78: Right: A CME classified as 'partial Halo CME' (asymmetric) observed on August 4th, 2011 by STEREO/SECCHI/COR2-A and -B. Left: The corresponding synthetic coronagraph images from calculations with the GCS modeling technique. 


\section{A.2.3 CME Class 04 - 'Ring' type CME}

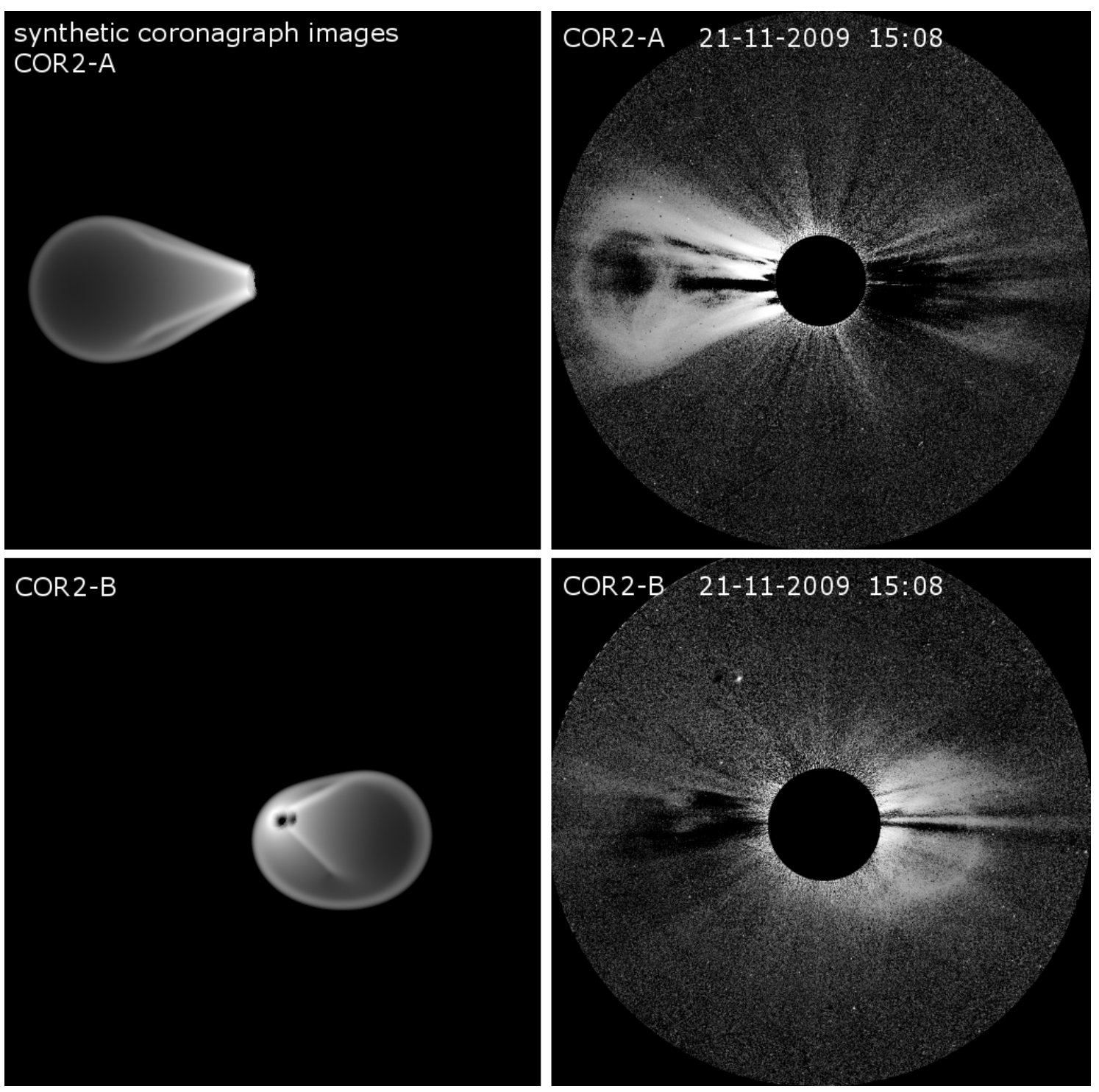

Fig. 79: Right: A CME classified as 'Ring type CME' observed on November 21th, 2009 by STEREO/SECCHI/COR2-A and -B. Left: The corresponding synthetic coronagraph images from calculations with the GCS modeling technique. 


\section{A.2.4 CME Class 05 - 'Jet-like' CME}
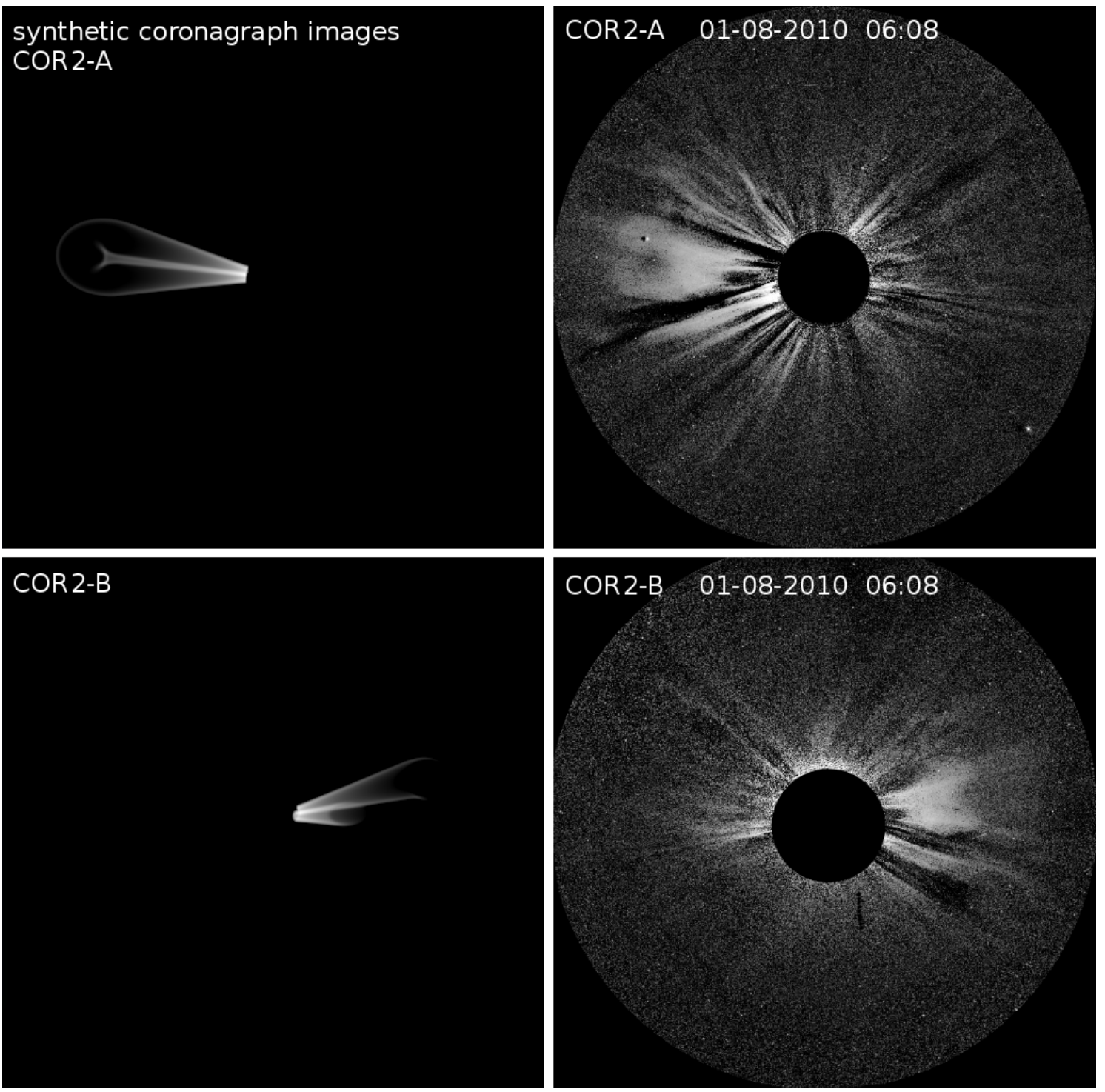

Fig. 80: Right: A CME classified as 'jet-like' CME observed on August 1st, 2010 by STEREO/SECCHI/COR2-A and -B. Left: The corresponding synthetic coronagraph images from calculations with the GCS modeling technique. 


\section{A.2.5 CME Class 08 - Distorted CME}
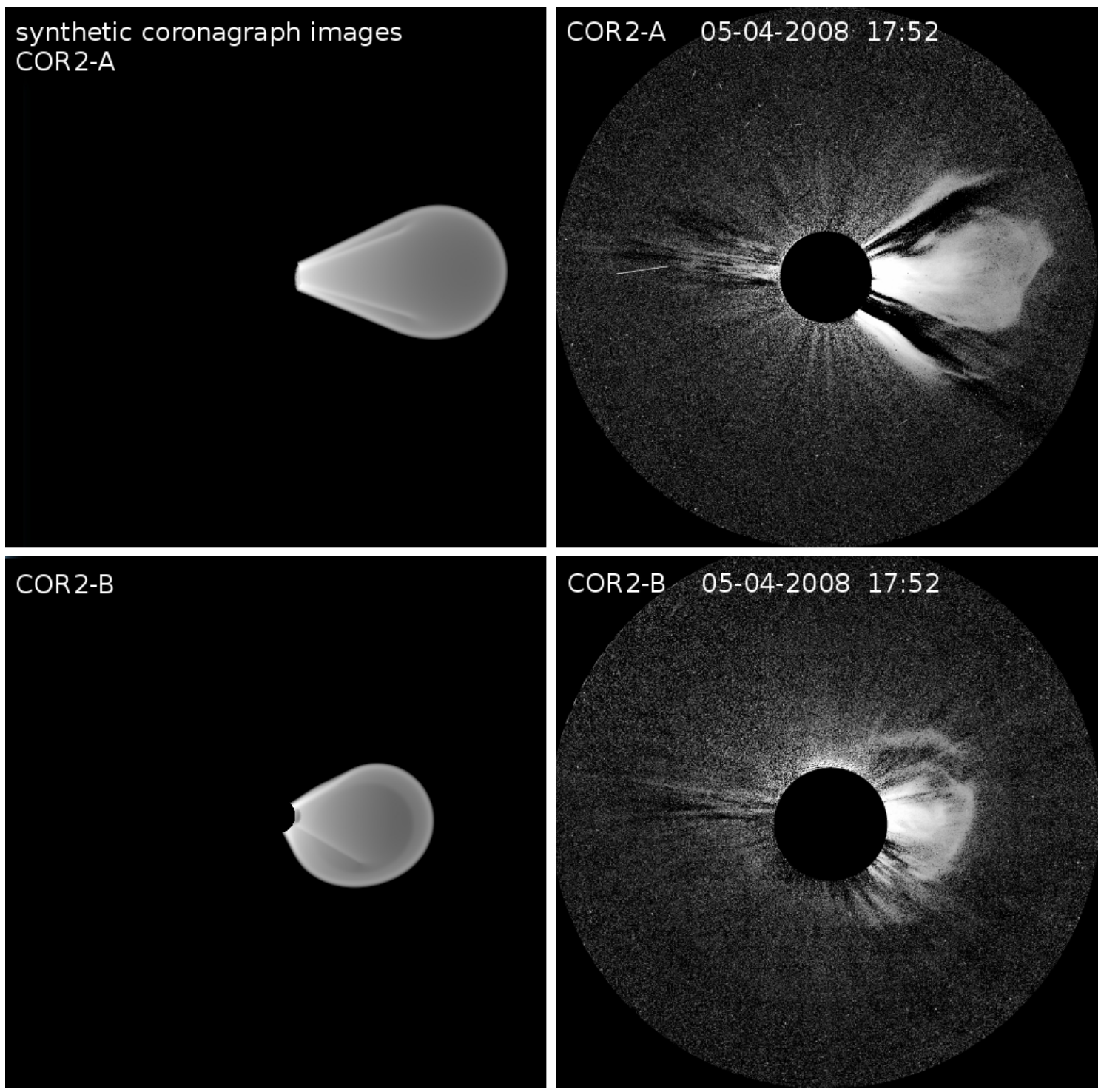

Fig. 81: Right: A CME classified as distorted CME observed on April 5th, 2008 by STEREO/SECCHI/COR2-A and -B. Left: The corresponding synthetic coronagraph images from calculations with the GCS modeling technique. 


\section{A.2.6 CME Class 10 - Non-rated CME}
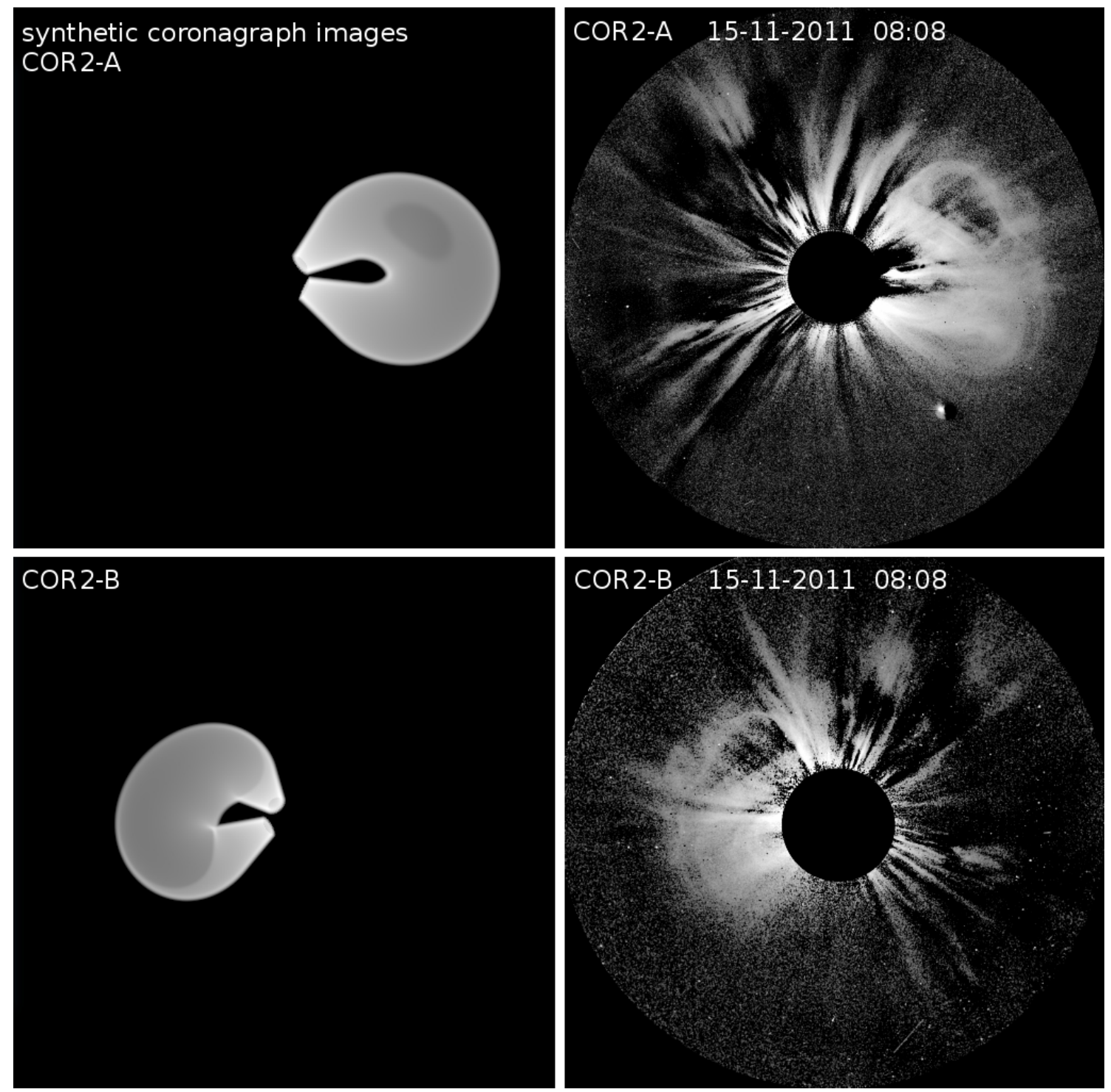

Fig. 82: Right: A non-rated CME observed on November 15th, 2008 by STEREO/SECCHI/COR2-A and -B. Left: The corresponding synthetic coronagraph images from calculations with the GCS modeling technique.

\section{A.3 GCS Aspect Ratio}

Several views of a demo GCS flux rope illustrate in Figure 83 the meaning of the aspect ratio parameter. In red is shown the radius 'a' for the circular cross section at the leading edge's apex within the tube section part. In light blue is presented the distance ' $r$ ' ranging from solar center to the center of the mentioned cross section. 


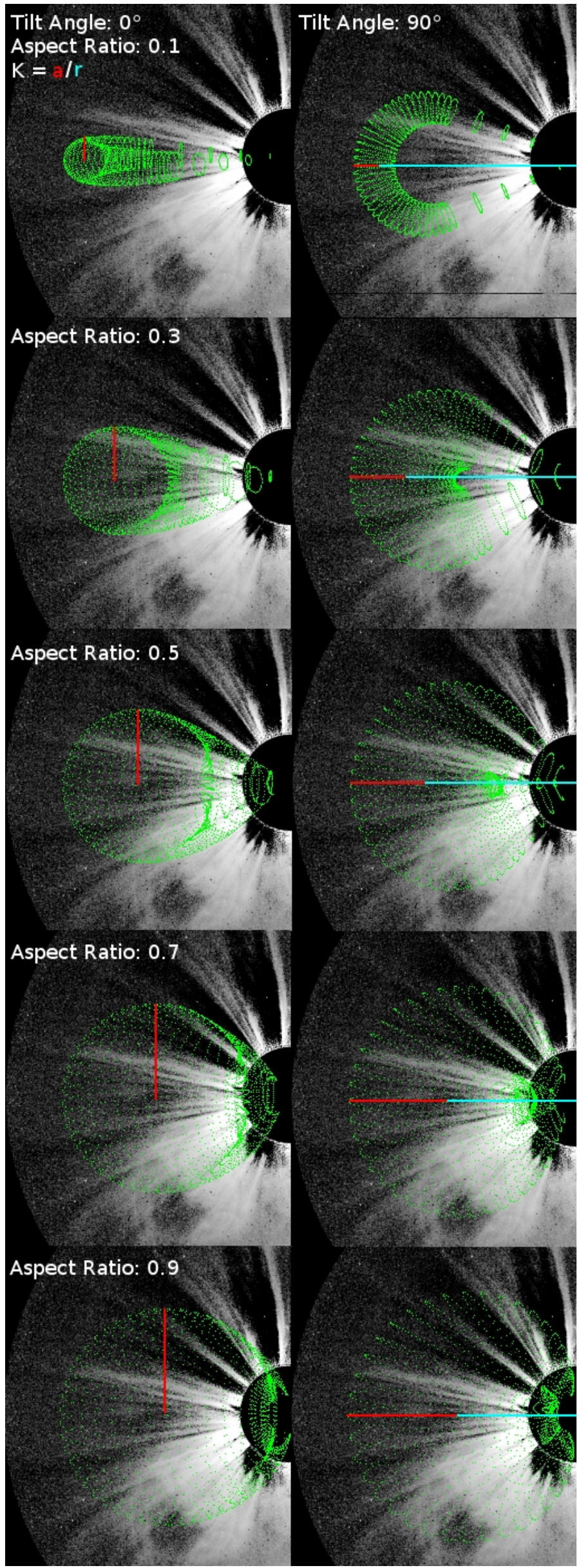

Fig. 83: These several sights of a demo GCS flux rope illustrate the meaning of the aspect ratio parameter. 


\section{A.4 The 'Best-of' CME List}

Tab. 13: 'Best-of' CME list with 264 CMEs detected with STEREO/SECCHI/COR2- A/B for the time period from January 2007 until December 2011. No indicates the CME number in the overall COR2 list which is available at www. affects-fp7.eu/cme-database. Date and time corresponds to the observation time with COR2-A and -B, respectively. $n$ stands for next day. $P A$ denotes the position angle corresponding to the COR2 field of view with $0^{\circ}$ pointing to the solar north and increasing counter-clockwise. The Flags indicate special features of a CME event $(\mathrm{b}=$ backside CME as seen from Earth, $\mathrm{pH}=$ partial Halo, $\mathrm{H}=$ Halo $\mathrm{CME}, \mathrm{d}=\mathrm{CME}$ with distorted leading edge, $\mathrm{n}=$ no fit available, $\mathrm{m}=$ missing data, $\mathrm{p}=$ pretty $\mathrm{CME}$ ). The last column comprises the separation angle between spacecraft STEREO-A and -B.

The 'Best-of' CME list is also available at www . affects-fp7.eu/cme-database.

\begin{tabular}{|c|c|c|c|c|c|c|c|}
\hline No & $\begin{array}{c}\text { Date } \\
\mathrm{y}-\mathrm{m}-\mathrm{d}\end{array}$ & $\begin{array}{c}\text { Time(A) } \\
\text { h:m:s }\end{array}$ & $\begin{array}{c}\mathrm{PA}(\mathrm{A}) \\
{[\mathrm{deg}]}\end{array}$ & $\begin{array}{c}\text { Time(B) } \\
\text { h:m:s }\end{array}$ & $\begin{array}{c}\mathrm{PA}(\mathrm{B}) \\
{[\mathrm{deg}]}\end{array}$ & Flag & $\begin{array}{c}\text { Sep.Angle } \\
\text { [deg] }\end{array}$ \\
\hline 5 & 20070124 & $15: 02: 40$ & 90 & $15: 02: 46$ & 90 & & 0.5 \\
\hline 7 & 20070130 & $11: 02: 40$ & 270 & $12: 02: 48$ & 270 & & 0.6 \\
\hline 20 & 20070312 & 00:02:40 & 90 & 00:03:01 & 90 & & 1.7 \\
\hline 25 & 20070331 & 09:52:30 & 270 & $09: 52: 58$ & 270 & & 3.0 \\
\hline 35 & 20070509 & $04: 22: 30$ & 90 & $04: 53: 14$ & 90 & & 7.2 \\
\hline 38 & 20070515 & $19: 22: 30$ & 45 & $20: 23: 17$ & 45 & & 8.1 \\
\hline 49 & 20070604 & $21: 22: 30$ & 270 & $03: 23: 24 n$ & 270 & & 11.3 \\
\hline 50 & 20070607 & $20: 22: 30$ & 225 & $21: 23: 25$ & 225 & & 11.8 \\
\hline 60 & 20070708 & $20: 22: 30$ & 90 & $21: 23: 32$ & 90 & & 17.5 \\
\hline 71 & 20070821 & 08:52:30 & 270 & 10:53:33 & 270 & & 26.3 \\
\hline 78 & 20071008 & $17: 22: 30$ & 270 & $16: 53: 22$ & 270 & & 35.2 \\
\hline 83 & 20071104 & $13: 52: 20$ & 45 & $20: 23: 02$ & 45 & & 39.0 \\
\hline 86 & 20071116 & $10: 52: 20$ & 225 & $12: 52: 57$ & 225 & & 40.4 \\
\hline 92 & 20071231 & $01: 22: 20$ & 135 & $01: 52: 42$ & 135 & & 44.0 \\
\hline 93 & 20080102 & $10: 22: 20$ & 90 & $11: 22: 41$ & 90 & & 44.1 \\
\hline 95 & 20080122 & $23: 52: 20$ & 180 & $23: 52: 38$ & 135 & & 44.9 \\
\hline 96 & 20080129 & $00: 22: 20$ & 270 & $00: 22: 37$ & 270 & & 45.1 \\
\hline 100 & 20080212 & $07: 52: 20$ & 225 & $09: 22: 36$ & 225 & & 45.6 \\
\hline 104 & 20080223 & $20: 52: 20$ & 45 & $17: 52: 37$ & 45 & & 45.9 \\
\hline 105 & 20080227 & $12: 52: 20$ & 270 & $10: 22: 37$ & 270 & & 46.0 \\
\hline 107 & 20080317 & $15: 52: 20$ & 270 & $12: 52: 40$ & 270 & & 46.8 \\
\hline 111 & 20080325 & $19: 22: 20$ & 90 & 19:52:42 & 90 & & 47.2 \\
\hline 113 & 20080405 & $16: 22: 20$ & 270 & $16: 52: 45$ & 270 & & 47.8 \\
\hline 121 & 20080426 & $14: 52: 20$ & 45 & $15: 52: 53$ & 315 & & 49.5 \\
\hline 125 & 20080517 & $10: 52: 20$ & 90 & $12: 23: 01$ & 90 & & 51.8 \\
\hline 130 & 20080601 & $23: 22: 20$ & 90 & - & - & & 53.9 \\
\hline 141 & 20080731 & $06: 22: 20$ & 270 & - & - & & 64.3 \\
\hline 145 & 20080908 & $02: 22: 20$ & 270 & 03:53:22 & 90 & b & 71.9 \\
\hline 156 & 20081017 & $09: 52: 20$ & 270 & $10: 53: 14$ & 90 & b & 79.1 \\
\hline 158 & 20081026 & $23: 52: 20$ & 270 & $22: 23: 11$ & 270 & & 80.7 \\
\hline 162 & 20081103 & $01: 22: 20$ & 45 & 01:53:09 & 315 & & 81.8 \\
\hline 167 & 20081113 & $16: 22: 20$ & 225 & $15: 23: 06$ & 135 & b & 83.3 \\
\hline 172 & 20081123 & $22: 22: 20$ & 270 & $04: 23: 02 n$ & 270 & & 84.6 \\
\hline 175 & 20081208 & $17: 22: 20$ & 315 & - & - & & 86.4 \\
\hline 176 & 20081212 & $08: 22: 20$ & 90 & $08: 22: 55$ & 270 & & 86.7 \\
\hline 179 & 20081227 & $06: 52: 20$ & 45 & $10: 52: 50$ & 315 & & 88.1 \\
\hline 186 & 20090114 & $06: 22: 00$ & 90 & $11: 22: 00$ & 270 & & 89.4 \\
\hline 188 & 20090117 & - & - & $14: 22: 24$ & 90 & & 89.6 \\
\hline 195 & 20090210 & 20:52:00 & $270 / 90$ & $01: 52: 19 \mathrm{n}$ & $225 / 90$ & $\mathrm{~b}$ ? & 90.9 \\
\hline 198 & 20090218 & $09: 22: 00$ & 90 & $15: 22: 19$ & $45-0-315$ & & 91.2 \\
\hline 207 & 20090316 & $14: 22: 00$ & $90 / 270$ & $12: 22: 19$ & $90 / 270$ & $\mathrm{~b}$ ? & 92.2 \\
\hline 208 & 20090318 & $00: 22: 00$ & 90 & $07: 22: 19$ & 315 & & 92.2 \\
\hline 213 & 20090326 & $09: 22: 00$ & 270 & $19: 22: 20$ & 135 & b & 92.6 \\
\hline 217 & 20090410 & $19: 22: 00$ & 270 & - & - & & 93.3 \\
\hline 226 & 20090423 & $00: 22: 00$ & $90 / 315$ & $00: 22: 26$ & 90 & $\mathrm{~b} ?$ & 94.1 \\
\hline 228 & 20090502 & $23: 22: 00$ & 270 & $20: 22: 28$ & 90 & $\mathrm{~b}$ & 94.8 \\
\hline 234 & 20090521 & - & - & $19: 22: 35$ & 135 & & 96.5 \\
\hline 243 & 20090613 & 13:52:00 & 90 & $14: 52: 43$ & 270 & & 99.2 \\
\hline 244 & 20090615 & 02:52:00n & 90 & $21: 22: 43$ & 270 & & 99.5 \\
\hline 245 & 20090616 & $16: 52: 00$ & 270 & $16: 52: 44$ & 270 & & 99.6 \\
\hline
\end{tabular}




\begin{tabular}{|c|c|c|c|c|c|c|c|}
\hline No & $\begin{array}{c}\text { Date } \\
\mathrm{y}-\mathrm{m}-\mathrm{d}\end{array}$ & $\begin{array}{c}\text { Time(A) } \\
\text { h:m:s }\end{array}$ & $\begin{array}{l}\mathrm{PA}(\mathrm{A}) \\
{[\mathrm{deg}]}\end{array}$ & $\begin{array}{c}\text { Time(B) } \\
\text { h:m:s }\end{array}$ & $\begin{array}{c}\mathrm{PA}(\mathrm{B}) \\
{[\mathrm{deg}]}\end{array}$ & Flag & $\begin{array}{l}\text { Sep.Angle } \\
\text { [deg] }\end{array}$ \\
\hline 259 & 20090723 & $06: 52: 49$ & 90 & 08:22:00 & 270 & & 105.3 \\
\hline 262 & 20090804 & $18: 22: 00$ & 90 & $18: 22: 49$ & 270 & & 107.5 \\
\hline 263 & 20090808 & $16: 52: 00$ & 90 & $01: 52: 49$ & 90 & & 108.1 \\
\hline 264 & 20090811 & $14: 22: 49$ & 90 & $20: 22: 00$ & 270 & & 108.7 \\
\hline 265 & 20090813 & 03:22:00 & 270 & - & - & & 109.0 \\
\hline 267 & 20090819 & 03:08:15 & 270 & 05:09:04 & 90 & b & 110.1 \\
\hline 271 & 20090903 & $12: 08: 15$ & 90 & 05:09:04 & 270 & & 112.9 \\
\hline 288 & 20091031 & $05: 08: 15$ & 90 & 05:09:04 & 270 & & 123.6 \\
\hline 291 & 20091108 & $05: 08: 15$ & 270 & 06:09:04 & 45 & b & 124.9 \\
\hline 293 & 20091115 & $13: 08: 15$ & 270 & - & - & & 126.1 \\
\hline 299 & 20091121 & $08: 08: 15$ & 90 & 11:09:04 & 270 & & 127.0 \\
\hline 307 & 20091216 & 03:08:15 & 90 & 04:08:58 & 270 & $\mathrm{~h}$ & 130.4 \\
\hline 325 & 20100201 & $23: 08: 15$ & $135-270$ & 22:09:04 & 225 & & 135.3 \\
\hline 333 & 20100211 & 21:08:15 & 90 & 23:08:41 & 270 & & 136.0 \\
\hline 337 & 20100214 & 08:08:15 & 45 & 05:08:40 & 270 & & 136.1 \\
\hline 347 & 20100225 & 01:08:15 & 135 & 02:08:38 & 225 & & 136.8 \\
\hline 350 & 20100228 & $18: 08: 15$ & 315 & 19:08:37 & 45 & $\mathrm{~b} ?$ & 137.0 \\
\hline 351 & 20100301 & 09:08:15 & $135-180$ & 08:08:37 & 225 & & 137.0 \\
\hline 359 & 20100309 & 08:08:15 & 270 & $12: 08: 36$ & 90 & b & 137.4 \\
\hline 365 & 20100314 & $13: 08: 15$ & 225 & $14: 08: 36$ & 90 & b & 137.7 \\
\hline 369 & 20100319 & 20:08:15 & 90 & 21:08:35 & 270 & & 138.0 \\
\hline 370 & 20100320 & 09:08:15 & 270 & $11: 08: 35$ & $315-135$ & $\mathrm{~b}$ ? & 138.0 \\
\hline 373 & 20100326 & $16: 08: 15$ & 90 & $17: 08: 35$ & 270 & & 138.3 \\
\hline 376 & 20100329 & 12:08:15 & 270 & 14:08:35 & 90 & b & 138.4 \\
\hline 377 & 20100330 & $12: 08: 15$ & $135 / 315$ & 14:08:35 & $45 / 225$ & b & 138.5 \\
\hline 379 & 20100403 & 11:08:15 & 135 & $12: 08: 35$ & 225 & $\mathrm{~h}$ & 138.6 \\
\hline 383 & 20100408 & 09:08:15 & 90 & $07: 08: 36$ & 270 & & 138.9 \\
\hline 386 & 20100411 & 09:08:15 & 225 & 11:08:36 & 135 & b & 139.0 \\
\hline 387 & 20100412 & 10:08:15 & 225 & $13: 08: 36$ & 135 & b & 139.1 \\
\hline 389 & 20100413 & $15: 08: 15$ & 45 & $14: 08: 36$ & 315 & & 139.1 \\
\hline 391 & 20100419 & 05:08:15 & 135 & $04: 08: 36$ & 225 & & 139.4 \\
\hline 392 & 20100419 & $23: 08: 15$ & 45 & $00: 08: 37 \mathrm{n}$ & 315 & & 139.4 \\
\hline 404 & 20100506 & 01:08:15 & 180 & 02:08:39 & 225 & & 140.4 \\
\hline 415 & 20100523 & 00:08:15 & 270 & 00:08:43 & 90 & b & 141.5 \\
\hline 416 & 20100523 & $21: 08: 15$ & 90 & $22: 08: 43$ & 270 & $\mathrm{~h}$ & 141.6 \\
\hline 418 & 20100524 & $17: 08: 15$ & 90 & $17: 08: 43$ & 270 & $\mathrm{~h}$ & 141.7 \\
\hline 426 & 20100604 & $16: 08: 15$ & 270 & $16: 08: 46$ & 90 & b & 142.6 \\
\hline 429 & 20100612 & $16: 08: 15$ & 45 & 18:08:49 & 0 & & 143.4 \\
\hline 432 & 20100615 & 03:08:15 & $225 / 45$ & 04:08:49 & $135 / 0$ & b & 143.6 \\
\hline 434 & 20100616 & $19: 08: 15$ & 90 & 20:08:50 & 270 & & 143.8 \\
\hline 435 & 20100619 & 03:08:15 & 315 & 04:08:51 & 45 & b & 144.0 \\
\hline 437 & 20100620 & $22: 08: 15$ & 90 & $23: 08: 15$ & 270 & & 144.2 \\
\hline 444 & 20100703 & $07: 08: 15$ & 270 & 08:08:55 & 90 & b & 145.6 \\
\hline $445 \mathrm{a}$ & 20100703 & $13: 08: 15$ & $180 / 90$ & $14: 08: 55$ & $225 / 315$ & & 145.7 \\
\hline $445 b$ & 20100703 & - & - & - & - & - & \\
\hline 448 & 20100705 & 21:08:15 & 315 & 20:08:56 & 45 & b & 145.9 \\
\hline 449 & 20100706 & 08:08:15 & $90 / 360$ & 09:08:56 & 270 & & 146.0 \\
\hline 464 & 20100801 & 04:08:15 & 90 & 05:09:04 & 270 & & 149.6 \\
\hline $465 a$ & 20100801 & 09:08:15 & 90 & 10:09:04 & 315 & & 149.6 \\
\hline $465 \mathrm{~b}$ & 20100801 & 09:54:00 & 45 & 10:09:04 & 315 & & 149.6 \\
\hline 470 & 20100807 & 20:08:15 & $90 / 225$ & 21:09:05 & 270 & $\mathrm{~h}$ & 150.6 \\
\hline 471 & 20100808 & $17: 08: 15$ & 225 & 18:09:06 & 135 & b & 150.7 \\
\hline 474 & 20100814 & $12: 08: 15$ & 135 & 13:09:07 & $225-270$ & $\mathrm{~h}$ & 151.7 \\
\hline 476 & 20100815 & $14: 08: 15$ & 45 & 16:09:07 & 315 & & 151.8 \\
\hline 479 & 20100818 & $06: 08: 15$ & 45 & 07:09:08 & 315 & & 152.3 \\
\hline 500 & 20100911 & $06: 08: 15$ & $45 / 225$ & 07:09:12 & $315 / 135$ & $\mathrm{~b}$ ? & 156.4 \\
\hline 511 & 20101001 & 05:08:15 & 315 & 06:09:14 & 45 & $\mathrm{~b}$ & 160.0 \\
\hline 515 & 20101006 & 10:08:15 & 90 & 14:09:14 & 270 & & 160.9 \\
\hline 516 & 20101007 & $15: 08: 15$ & 0 & 16:09:14 & 0 & & 161.1 \\
\hline 519 & 20101011 & 05:08:15 & 90 & 06:09:14 & 270 & & 161.8 \\
\hline 525 & 20101026 & $15: 08: 15$ & $135 / 315$ & 16:09:14 & $225 / 0$ & & 164.6 \\
\hline 528 & 20101028 & $16: 08: 15$ & 225 & $15: 09: 13$ & 90 & b & 164.9 \\
\hline 546 & 20101116 & $14: 08: 15$ & 135 & 15:09:11 & 225 & & 168.3 \\
\hline 551 & 20101124 & 09:08:15 & 135 & 10:09:10 & 270 & & 169.6 \\
\hline 552 & 20101126 & 00:08:15 & 225 & 01:09:10 & 135 & b & 169.9 \\
\hline 558 & 20101202 & $13: 08: 15$ & 270 & 16:09:09 & 90 & b & 171.0 \\
\hline
\end{tabular}




\begin{tabular}{|c|c|c|c|c|c|c|c|}
\hline No & $\begin{array}{c}\text { Date } \\
\mathrm{y}-\mathrm{m}-\mathrm{d}\end{array}$ & $\begin{array}{c}\text { Time(A) } \\
\text { h:m:s }\end{array}$ & $\begin{array}{c}\mathrm{PA}(\mathrm{A}) \\
{[\mathrm{deg}]}\end{array}$ & $\begin{array}{c}\text { Time(B) } \\
\text { h:m:s }\end{array}$ & $\begin{array}{c}\mathrm{PA}(\mathrm{B}) \\
{[\mathrm{deg}]}\end{array}$ & Flag & $\begin{array}{c}\text { Sep.Angle } \\
\text { [deg] }\end{array}$ \\
\hline 561 & 20101206 & $00: 08: 15 n$ & 135 & $23: 09: 08$ & 225 & & 171.7 \\
\hline $566 \mathrm{a}$ & 20101212 & 08:08:15 & $45 / 135$ & 10:09:06 & $225 / 315$ & $\mathrm{pH}$ & 172.5 \\
\hline $566 \mathrm{~b}$ & 20101212 & - & - & - & - & - & - \\
\hline $568 \mathrm{a}$ & 20101214 & $18: 08: 15$ & 45 & 19:09:06 & 315 & & 172.9 \\
\hline $568 \mathrm{~b}$ & 20101214 & - & - & - & - & - & - \\
\hline 574 & 20101223 & $12: 08: 15$ & 90 & 13:09:04 & 270 & d & 174.2 \\
\hline 576 & 20101226 & 09:08:15 & 225 & 10:09:03 & 135 & $\mathrm{~b}$ & 174.7 \\
\hline 587 & 20110109 & $23: 08: 15$ & 45 & $01: 08: 59 n$ & 315 & & 176.7 \\
\hline 590 & 20110112 & $10: 08: 15$ & $90-270$ & $11: 08: 58$ & $90-270$ & $\mathrm{pH}$ & 177.0 \\
\hline 595 & 20110118 & $05: 08: 15$ & 315 & $05: 08: 57$ & 45 & $\mathrm{n}$ & 177.8 \\
\hline 597 & 20110120 & $12: 08: 15$ & 225 & $14: 08: 57$ & 135 & $\mathrm{~b}$ ? & 178.1 \\
\hline 603 & 20110124 & $04: 08: 15$ & 90 & $05: 08: 55$ & 270 & & 178.5 \\
\hline 606 & 20110126 & $02: 08: 15$ & 45 & 03:08:54 & 315 & & 178.7 \\
\hline 608 & 20110128 & $02: 08: 15$ & 315 & $02: 08: 53$ & 45 & $\mathrm{pH}, \mathrm{b}$ & 179.0 \\
\hline 612 & 20110130 & 10:08:15 & $135 / 45$ & 10:08:53 & $225 / 315$ & & 179.2 \\
\hline 614 & 20110201 & $21: 08: 15$ & 315 & $22: 08: 52$ & 45 & & 179.4 \\
\hline 615 & 20110202 & 03:08:15 & 315 & 03:08:52 & 45 & $\mathrm{~b}$ & 179.5 \\
\hline 620 & 20110210 & $03: 08: 15$ & 270 & 04:08:50 & 90 & $\mathrm{~b}$ & 179.5 \\
\hline 622 & 20110214 & $18: 08: 15$ & 90 & 19:08:48 & 270 & & 179.1 \\
\hline 623 & 20110215 & $02: 08: 15$ & 90 & 03:08:48 & 270 & & 179.1 \\
\hline 624 & 20110216 & $05: 08: 15$ & 45 & 04:08:48 & 315 & & 179.0 \\
\hline 628 & 20110224 & $10: 08: 15$ & 180 & 11:08:46 & 90 & $\mathrm{pH}$ & 178.3 \\
\hline 631 & 20110226 & $17: 08: 15$ & 315 & $17: 08: 45$ & 45 & $\mathrm{mb}$ & 178.1 \\
\hline 635 & 20110303 & $04: 08: 15$ & 135 & $06: 08: 44$ & 225 & & 177.7 \\
\hline 636 & 20110303 & $23: 08: 15$ & $45-90$ & 23:08:44 & $270-315$ & $\mathrm{n}$ & 177.7 \\
\hline 645 & 20110307 & $15: 08: 15$ & 45 & $15: 08: 43$ & 315 & $\mathrm{mn}$ & 177.4 \\
\hline 646 & 20110307 & $20: 08: 15$ & 45 & 21:08:43 & 315 & $\mathrm{~h}$ & 177.3 \\
\hline 647 & 20110308 & $05: 08: 15$ & 360 & 05:08:43 & 360 & hn? & 177.3 \\
\hline 648 & 20110308 & $21: 08: 15$ & 180 & $22: 08: 42$ & 180 & $\mathrm{pH} ?$ & 177.2 \\
\hline 651 & 20110312 & $02: 08: 15$ & 225 & 03:08:42 & 135 & $\mathrm{~b}$ & 177.0 \\
\hline 653 & 20110314 & 09:08:15 & 45 & 09:08:41 & 315 & & 176.8 \\
\hline 655 & 20110316 & $20: 08: 15$ & 45 & $20: 08: 41$ & 315 & & 176.7 \\
\hline 656 & 20110317 & $13: 08: 15$ & 225 & 13:08:41 & 135 & $\mathrm{~b}$ & 176.6 \\
\hline 661 & 20110321 & 03:08:15 & 315 & 03:08:40 & 45 & $\mathrm{pH}, \mathrm{b} ?$ & 176.4 \\
\hline 665 & 20110323 & $19: 08: 15$ & 315 & 19:08:39 & 45 & $\mathrm{~b}$ & 176.2 \\
\hline 667 & 20110324 & $13: 08: 15$ & 315 & $13: 08: 39$ & 45 & bn? & 176.2 \\
\hline 668 & 20110325 & 08:08:15 & $90 / 270$ & 08:08:39 & $270 / 90$ & & 176.1 \\
\hline 669 & 20110326 & $06: 08: 15$ & 315 & $07: 08: 39$ & 45 & $\mathrm{~h} ? \mathrm{~b}$ ? & 176.1 \\
\hline 673 & 20110328 & $17: 08: 15$ & 315 & 17:08:39 & 45 & $\mathrm{~b}$ & 176.0 \\
\hline 675 & 20110329 & $21: 08: 15$ & 360 & $21: 08: 38$ & 360 & $\mathrm{~h}$ & 175.9 \\
\hline 679 & 20110403 & 01:08:15 & 135 & 01:08:38 & 225 & & 175.6 \\
\hline 680 & 20110404 & $04: 08: 15$ & 90 & 05:08:38 & 270 & & 175.5 \\
\hline 681 & 20110404 & $04: 08: 15$ & 315 & $05: 08: 38$ & 45 & $\mathrm{~b}$ & 175.5 \\
\hline 682 & 20110407 & $07: 08: 15$ & 90 & 08:08:37 & 270 & & 175.4 \\
\hline 683 & 20110407 & $12: 08: 15$ & 225 & $13: 08: 37$ & 135 & & 175.4 \\
\hline 684 & 20110407 & $22: 08: 15$ & 270 & $22: 08: 37$ & 90 & & 175.4 \\
\hline 689 & 20110412 & $01: 08: 15$ & 45 & 01:08:37 & 315 & & 175.1 \\
\hline 696 & 20110417 & $02: 08: 15$ & 45 & $02: 08: 37$ & 315 & & 174.9 \\
\hline 697 & 20110417 & $16: 08: 15$ & 270 & $16: 08: 36$ & 90 & $\mathrm{~b}$ ? & 174.9 \\
\hline 703 & 20110424 & $20: 08: 15$ & 315 & $20: 08: 36$ & 45 & $\mathrm{~b}$ ? & 174.5 \\
\hline 706 & 20110427 & 08:08:15 & 315 & 08:08:36 & 45 & $\mathrm{~b}$ & 174.4 \\
\hline 711 & 20110501 & $17: 08: 15$ & 45 & $17: 08: 36$ & 315 & & 174.1 \\
\hline 716 & 20110506 & $10: 08: 15$ & $270-90$ & $10: 08: 36$ & $270-90$ & $\mathrm{pH}, \mathrm{b} ?$ & 173.9 \\
\hline 721 & 20110511 & $04: 08: 15$ & 90 & 04:08:37 & 270 & & 173.6 \\
\hline 723 & 20110512 & 18:08:15 & $225 / 45$ & $21: 08: 37$ & $135 / 315$ & $\mathrm{~m}$ & 173.6 \\
\hline 732 & 20110518 & $19: 08: 15$ & 360 & $20: 08: 37$ & 360 & $\mathrm{~h}$ & 173.3 \\
\hline 733 & 20110519 & 05:08:15 & 45 & 06:08:37 & 315 & & 173.2 \\
\hline 735 & 20110520 & $22: 08: 15$ & 270 & $22: 08: 37$ & 90 & $\mathrm{~b}$ & 173.2 \\
\hline 743 & 20110529 & $12: 08: 15$ & 90 & 12:08:39 & 270 & $\mathrm{mh}$ & 172.6 \\
\hline 748 & 20110601 & 04:08:15 & 90 & 06:08:39 & 270 & & 172.5 \\
\hline 750 & 20110601 & $18: 08: 15$ & 90 & 19:08:39 & 270 & & 172.5 \\
\hline 751 & 20110602 & 08:08:15 & 90 & 08:08:39 & 270 & & 172.4 \\
\hline 753 & 20110603 & $05: 08: 15$ & 135 & 11:08:39 & 225 & & 172.3 \\
\hline 754 & 20110604 & $07: 08: 15$ & 360 & 07:08:40 & 360 & $\mathrm{~b}$ & 172.3 \\
\hline 755 & 20110604 & $22: 08: 15$ & 360 & $22: 08: 40$ & 360 & $\mathrm{~b}$ & 172.3 \\
\hline 756 & 20110605 & $06: 08: 15$ & 45 & 07:08:40 & 315 & & 172.2 \\
\hline
\end{tabular}




\begin{tabular}{|c|c|c|c|c|c|c|c|}
\hline No & $\begin{array}{c}\text { Date } \\
\mathrm{y}-\mathrm{m}-\mathrm{d}\end{array}$ & $\begin{array}{c}\text { Time(A) } \\
\text { h:m:s }\end{array}$ & $\begin{array}{c}\mathrm{PA}(\mathrm{A}) \\
{[\mathrm{deg}]}\end{array}$ & $\begin{array}{c}\text { Time(B) } \\
\text { h:m:s }\end{array}$ & $\begin{array}{c}\mathrm{PA}(\mathrm{B}) \\
{[\mathrm{deg}]}\end{array}$ & Flag & $\begin{array}{c}\text { Sep.Angle } \\
\text { [deg] }\end{array}$ \\
\hline 757 & 20110606 & 08:08:15 & $90 / 180$ & 09:08:40 & $270 / 180$ & & 172.1 \\
\hline 758 & 20110607 & $07: 08: 15$ & 135 & $07: 08: 40$ & 225 & & 172.1 \\
\hline 760 & 20110608 & 08:08:15 & 315 & 08:08:40 & 45 & $\mathrm{~b}$ & 172.0 \\
\hline 762 & 20110609 & 09:08:15 & 0 & 10:08:40 & 0 & & 171.9 \\
\hline 769 & 20110612 & 18:08:15 & 360 & 20:08:41 & 360 & $\mathrm{~h}$ & 171.7 \\
\hline 770 & 20110613 & 05:08:15 & 270 & 05:08:41 & 90 & $\mathrm{db}$ & 171.7 \\
\hline 772 & 20110614 & 08:08:15 & 90 & 08:08:41 & 270 & & 171.6 \\
\hline 776 & 20110620 & 19:08:15 & 270 & 19:08:43 & 90 & $\mathrm{~b}$ & 171.2 \\
\hline 777 & 20110621 & 03:08:15 & 90 & 03:08:43 & 270 & & 171.1 \\
\hline 789 & 20110704 & $16: 54: 00$ & 225 & $19: 54: 32$ & 135 & & 170.0 \\
\hline 794 & 20110709 & 00:54:00 & 90 & $00: 54: 33$ & 270 & $\mathrm{~d}$ & 169.5 \\
\hline 796 & 20110709 & $21: 54: 00$ & 315 & $21: 54: 33$ & 45 & $\mathrm{~b}$ & 169.5 \\
\hline 797 & 20110710 & $13: 54: 00$ & 0 & $13: 54: 33$ & 0 & & 169.4 \\
\hline 809 & 20110720 & $12: 54: 00$ & 315 & $12: 54: 36$ & 45 & b & 168.4 \\
\hline 810 & 20110721 & $17: 54: 00$ & 225 & $20: 54: 36$ & 135 & & 168.3 \\
\hline 812 & 20110724 & $17: 54: 00$ & 45 & $19: 54: 37$ & 315 & $\mathrm{n}$ & 168.0 \\
\hline 813 & 20110725 & $17: 54: 00$ & 270 & $17: 54: 38$ & 90 & $\mathrm{~b}$ & 167.9 \\
\hline 817 & 20110728 & $17: 54: 00$ & 225 & $19: 54: 38$ & 135 & & 167.5 \\
\hline 818 & 20110802 & $06: 54: 00$ & 90 & $06: 54: 40$ & 270 & $\mathrm{mn}$ & 167.0 \\
\hline 821 & 20110803 & $13: 54: 00$ & 90 & $13: 54: 40$ & 270 & & 166.8 \\
\hline 822 & 20110804 & 04:54:00 & $45-90$ & $04: 54: 40$ & $270-315$ & $\mathrm{pH}$ & 166.7 \\
\hline 823 & 20110804 & 06:54:00 & 315 & $06: 54: 40$ & 45 & $\mathrm{~b}$ & 166.7 \\
\hline 831 & 20110811 & $11: 54: 00$ & $45-90$ & $11: 54: 43$ & $270-315$ & $\mathrm{~h}$ & 165.8 \\
\hline 832 & 20110812 & 08:54:00 & 45 & $08: 54: 43$ & 315 & $\mathrm{mn}$ & 165.7 \\
\hline 834 & 20110813 & $10: 54: 00$ & 315 & $10: 54: 43$ & 45 & $\mathrm{~b}$ & 165.6 \\
\hline 836 & 20110816 & 08:54:00 & 270 & $08: 54: 44$ & 90 & $\mathrm{mn}$ & 165.2 \\
\hline 841 & 20110824 & 00:54:00 & 270 & $00: 54: 46$ & 90 & $\mathrm{~b} ?$ & 164.1 \\
\hline 844 & 20110827 & $15: 54: 00$ & 315 & $15: 54: 47$ & 45 & $\mathrm{~b}$ & 163.7 \\
\hline 845 & 20110828 & 07:54:00 & 315 & $07: 54: 47$ & 45 & $\mathrm{~b} ?$ & 163.5 \\
\hline 851 & 20110902 & 07:54:00 & $225 / 90$ & $10: 54: 49$ & $135 / 270$ & & 162.8 \\
\hline 861 & 20110906 & $22: 54: 00$ & 45 & $22: 54: 50$ & 315 & & 162.2 \\
\hline 865 & 20110907 & $18: 54: 00$ & 270 & $19: 54: 50$ & 90 & $\mathrm{n}$ & 162.0 \\
\hline 867 & 20110907 & $23: 54: 00$ & 45 & $23: 54: 50$ & 315 & & 162.0 \\
\hline 870 & 20110908 & $22: 54: 00$ & 315 & $22: 54: 50$ & 45 & $\mathrm{pH}$ & 161.9 \\
\hline $871 \mathrm{a}$ & 20110909 & 07:54:00 & $90 / 45$ & 09:54:50 & $270 / 315$ & & 161.7 \\
\hline $871 b$ & 20110909 & - & - & - & - & - & 161.7 \\
\hline 873 & 20110910 & 03:54:00 & 45 & $03: 54: 51$ & 315 & & 161.6 \\
\hline 874 & 20110910 & $10: 54: 00$ & 90 & $11: 54: 51$ & 270 & & 161.6 \\
\hline 879 & 20110913 & $23: 54: 00$ & 45 & $23: 54: 52$ & 315 & $\mathrm{p}$ & 161.1 \\
\hline 881 & 20110914 & 21:54:00 & 90 & $23: 54: 52$ & 270 & & 160.9 \\
\hline 890 & 20110921 & $22: 54: 00$ & 360 & $22: 54: 53$ & 360 & h & 159.8 \\
\hline 891 & 20110922 & 11:54:00 & 360 & $11: 54: 53$ & 360 & $\mathrm{~h}$ & 159.7 \\
\hline 897 & 20110924 & $13: 54: 00$ & 360 & $13: 54: 54$ & 360 & $\mathrm{~h}$ & 159.3 \\
\hline 898 & 20110924 & 19:54:00 & $45-135$ & $19: 54: 54$ & $225-315$ & $\mathrm{n} ?$ & 159.3 \\
\hline 900 & 20110925 & 05:54:00 & 135 & $05: 54: 54$ & 270 & $\mathrm{n}$ & 159.2 \\
\hline 901 & 20110925 & 08:54:00 & $135 / 0$ & $10: 54: 54$ & $225 / 0$ & & 159.2 \\
\hline $906 a$ & 20110929 & $19: 54: 00$ & $225 / 315$ & $19: 54: 55$ & $45 / 135$ & & 158.5 \\
\hline $906 \mathrm{~b}$ & 20110929 & 19:54:00 & $225 / 315$ & $19: 54: 55$ & $45 / 135$ & & 158.5 \\
\hline 907 & 20110930 & 01:54:00 & 315 & $01: 54: 55$ & 45 & $\mathrm{~b}$ & 158.3 \\
\hline 911 & 20111001 & 21:54:00 & 360 & $21: 54: 55$ & 360 & $\mathrm{~h}$ & 158.2 \\
\hline 919 & 20111005 & $13: 54: 00$ & 135 & $16: 54: 56$ & 225 & & 157.5 \\
\hline 923 & 20111010 & 04:54:00 & 270 & $04: 54: 57$ & 90 & $\mathrm{~b}$ & 156.6 \\
\hline 928 & 20111014 & $12: 54: 00$ & 270 & $12: 54: 57$ & 90 & $\mathrm{~b}$ & 155.9 \\
\hline 939 & 20111021 & $14: 54: 00$ & 135 & $21: 54: 58$ & 225 & $\mathrm{mn}$ & 154.7 \\
\hline 940 & 20111022 & 00:24:00 & 45 & $00: 24: 58$ & $315-0$ & $\mathrm{mn}$ & 154.5 \\
\hline 941 & 20111022 & $10: 54: 00$ & $315-90$ & $11: 54: 58$ & $315-90$ & & 154.5 \\
\hline 950 & 20111026 & $12: 54: 00$ & 90 & $11: 54: 58$ & 270 & $\mathrm{~m}$ & 153.8 \\
\hline 957 & 20111028 & 21:54:00 & 225 & $21: 54: 59$ & 135 & $\mathrm{~b}$ & 153.4 \\
\hline 962 & 20111031 & $16: 54: 00$ & 45 & $17: 54: 59$ & 315 & & 152.9 \\
\hline 967 & 20111103 & $22: 54: 00$ & 270 & $22: 54: 59$ & 90 & $\mathrm{~b}$ & 152.4 \\
\hline 968 & 20111104 & 01:54:00 & 270 & 02:54:59 & 90 & & 152.2 \\
\hline 969 & 20111105 & 02:54:00 & 45 & $03: 54: 59$ & 315 & & 152.0 \\
\hline 971 & 20111108 & 00:54:00 & 45 & $00: 54: 59$ & 315 & & 151.5 \\
\hline 974 & 20111109 & 09:54:00 & 135 & 09:54:59 & 225 & & 151.3 \\
\hline 975 & 20111109 & $13: 54: 00$ & 45 & $13: 54: 59$ & 315 & $\mathrm{~h}$ & 151.3 \\
\hline 980 & 20111112 & 19:54:00 & 360 & $20: 54: 59$ & 360 & $\mathrm{~h}$ & 150.7 \\
\hline
\end{tabular}




\begin{tabular}{rccccccr}
\hline No & $\begin{array}{c}\text { Date } \\
\text { y-m-d }\end{array}$ & $\begin{array}{c}\text { Time(A) } \\
\text { h:m:s }\end{array}$ & $\begin{array}{c}\text { PA(A) } \\
{[\mathrm{deg}]}\end{array}$ & $\begin{array}{c}\text { Time(B) } \\
\text { h:m:s }\end{array}$ & $\begin{array}{c}\text { PA(B) } \\
{[\mathrm{deg}]}\end{array}$ & Flag & $\begin{array}{c}\text { Sep.Angle } \\
{[\mathrm{deg}]}\end{array}$ \\
\hline 981 & 20111113 & $18: 54: 00$ & $270-315$ & $18: 54: 59$ & $45-90$ & $\mathrm{~b}$ & 150.5 \\
983 & 20111114 & $20: 54: 00$ & 135 & $21: 54: 59$ & 225 & & 150.4 \\
985 & 20111115 & $05: 54: 00$ & 270 & $06: 54: 59$ & 90 & $\mathrm{~b}$ & 150.2 \\
1002 & 20111122 & $22: 54: 00$ & 45 & $22: 54: 59$ & 0 & & 148.9 \\
1007 & 20111126 & $07: 54: 00$ & 90 & $07: 54: 59$ & 315 & $\mathrm{~h}$ & 148.2 \\
1013 & 20111129 & $23: 54: 00$ & 225 & $00: 54: 59$ & 135 & $\mathrm{~b}$ & 147.6 \\
1017 & 20111202 & $19: 54: 00$ & 0 & $20: 54: 58$ & 0 & & 147.1 \\
1024 & 20111207 & $05: 54: 00$ & 315 & $05: 54: 58$ & 45 & & 146.2 \\
1027 & 20111208 & $12: 54: 00$ & 180 & $16: 54: 58$ & 225 & $\mathrm{mn}$ & 146.0 \\
1050 & 20111221 & $03: 54: 00$ & 225 & $03: 54: 56$ & 135 & $\mathrm{~b}$ & 143.7 \\
1053 & 20111223 & $03: 54: 00$ & $315 / 225$ & $03: 54: 56$ & 90 & $\mathrm{~b} ?$ & 143.4 \\
1058 & 20111225 & $01: 54: 00$ & $45-90$ & $01: 54: 55$ & 315 & & 143.0 \\
1061 & 20111226 & $11: 54: 00$ & $45-90$ & $12: 54: 55$ & $270-315$ & & 142.9 \\
1067 & 20111229 & $07: 54: 00$ & 45 & $04: 54: 55$ & 315 & & 142.4 \\
1068 & 20111229 & $17: 54: 00$ & 45 & $17: 54: 55$ & 45 & $\mathrm{~h}$ & 142.4 \\
\hline
\end{tabular}

One event was found in the synoptic movies subsequently, it was labelled with $465 \mathrm{~b}$ and the previously found event 465 with $465 \mathrm{a}$.

\section{A.5 The GCS Modeling Results List}

Tab. 14: The fit results of the 241 modelled CME events which are listed in Table 13. No indicates the CME number in the overall COR2 list, the six following parameters are the same as introduced in Section 5.2 and summarised in Table 7. The column next to last denotes the time stamp of the COR2 images used for fitting. The Flags indicate special features of a CME event $(\mathrm{h}=$ Halo $\mathrm{CME}, \mathrm{n}=$ nice fit, $\mathrm{d}=\mathrm{CME}$ with distorted leading edge, $\mathrm{c}=$ classical typical CME and fit, $\mathrm{m}=$ missing FITS files).

\begin{tabular}{|c|c|c|c|c|c|c|c|c|}
\hline No & $\begin{array}{c}\phi \\
{[\mathrm{deg}]}\end{array}$ & $\begin{array}{c}\theta \\
{[\mathrm{deg}]}\end{array}$ & $\begin{array}{c}\gamma \\
\text { [deg] }\end{array}$ & $\begin{array}{c}\mathrm{h}_{\text {front }} \\
\mathrm{r}_{\odot} \\
\end{array}$ & $\begin{array}{l}\kappa \\
- \\
\end{array}$ & $\begin{array}{c}\alpha \\
{[\operatorname{deg}]}\end{array}$ & $\begin{array}{l}\text { Time stamp } \\
\text { y-m-d h-m-s }\end{array}$ & $\begin{array}{r}\text { Flag } \\
- \\
\end{array}$ \\
\hline 5 & 3.355 & 6.149 & 90.000 & 21.500 & 0.204 & 15.652 & 20070124-18:02:40 & \\
\hline 7 & 195.653 & -2.236 & 7.826 & 13.000 & 0.238 & 17.330 & 20070130-14:02:40 & \\
\hline 20 & 163.231 & 1.678 & -7.828 & 11.000 & 0.241 & 24.595 & 20070312-11:02:40 & \\
\hline 25 & 83.848 & 5.031 & 7.826 & 12.143 & 0.327 & 27.112 & $20070331-16: 52: 30$ & \\
\hline 35 & 88.322 & 1.118 & -17.330 & 13.143 & 0.425 & 10.061 & $20070509-11: 22: 30$ & \\
\hline 38 & 39.132 & 13.415 & 51.986 & 12.857 & 0.345 & 28.229 & 20070516-00:52:30 & \\
\hline 49 & 319.752 & -10.062 & 1.678 & 13.571 & 0.453 & 27.950 & $20070605-08: 52: 30$ & \\
\hline 50 & 240.372 & -11.740 & -10.062 & 13.500 & 0.259 & 17.049 & 20070608-02:52:30 & \\
\hline 60 & 54.781 & -8.384 & 6.709 & 10.357 & 0.225 & 24.595 & 20070708-23:52:30 & \\
\hline 71 & 24.595 & -13.415 & 32.981 & 12.500 & 0.238 & 13.136 & 20070821-13:52:30 & \\
\hline 78 & 40.244 & 9.502 & 0.560 & 14.214 & 0.299 & 12.578 & 20071009-00:22:30 & \\
\hline 83 & 309.690 & 12.298 & -27.392 & 14.071 & 0.296 & 18.447 & $20071104-22: 22: 00$ & \\
\hline 86 & 323.104 & -14.535 & 5.591 & 11.643 & 0.324 & 18.447 & 20071116-15:22:00 & \\
\hline 92 & 239.256 & -20.686 & -12.298 & 15.000 & 0.680 & 5.590 & 20071231-03:22:20 & \\
\hline 93 & 249.318 & -8.384 & 12.857 & 13.714 & 0.425 & 9.503 & 20080102-13:22:00 & \\
\hline 95 & 226.955 & -25.155 & -65.405 & 17.214 & 0.391 & 9.223 & 20080123-06:52:00 & \\
\hline 96 & 52.546 & 2.795 & 5.031 & 12.072 & 0.225 & 10.341 & 20080129-07:22:20 & \\
\hline 100 & 213.541 & -26.273 & 3.913 & 12.143 & 0.367 & 17.888 & 20080212-15:22:00 & \\
\hline 104 & 205.715 & 19.006 & 25.155 & 15.214 & 0.238 & 11.739 & 20080224-05:52:00 & \\
\hline 105 & 0.000 & 2.236 & 3.913 & 15.143 & 0.274 & 15.932 & $20080227-15: 22: 00$ & \\
\hline 107 & 71.554 & 6.709 & 35.217 & 10.929 & 0.201 & 7.826 & $20080317-17: 52: 00$ & \\
\hline 111 & 197.888 & -6.709 & -7.828 & 12.572 & 0.493 & 21.522 & $20080325-20: 52: 00$ & \\
\hline 113 & 260.492 & 2.236 & -17.330 & 12.857 & 0.357 & 8.663 & $20080405-17: 52: 20$ & \\
\hline 121 & 203.479 & 5.591 & 14.535 & 15.143 & 0.219 & 13.975 & $20080426-17: 52: 00$ & \\
\hline 125 & 263.851 & -11.740 & 0.000 & 12.786 & 0.379 & 11.739 & $20080517-12: 22: 00$ & \\
\hline 130 & 49.194 & -3.353 & 0.000 & 14.500 & 0.388 & 21.242 & $20080602-09: 22: 00$ & \\
\hline 141 & 173.293 & 11.740 & 0.000 & 13.571 & 0.311 & 16.211 & 20080731-23:52:01 & \\
\hline 145 & 90.558 & 0.000 & 6.149 & 16.214 & 0.364 & 7.268 & 20080908-11:22:00 & \\
\hline 156 & 270.558 & -10.062 & -42.484 & 17.500 & 0.419 & 5.870 & $20081017-16: 22: 00$ & \\
\hline
\end{tabular}




\begin{tabular}{|c|c|c|c|c|c|c|c|c|}
\hline No & $\begin{array}{c}\phi \\
{[\mathrm{deg}]}\end{array}$ & $\begin{array}{c}\theta \\
{[\mathrm{deg}]}\end{array}$ & $\begin{array}{c}\gamma \\
{[\mathrm{deg}]}\end{array}$ & $\begin{array}{c}\mathrm{h}_{\text {front }} \\
\mathrm{r}_{\odot} \\
\end{array}$ & $\begin{array}{l}\kappa \\
- \\
\end{array}$ & $\begin{array}{c}\alpha \\
{[\mathrm{deg}]}\end{array}$ & $\begin{array}{l}\text { Time stamp } \\
\mathrm{y}-\mathrm{m}-\mathrm{d} \text { h-m-s }\end{array}$ & $\begin{array}{r}\text { Flag } \\
- \\
\end{array}$ \\
\hline 158 & 8.942 & -0.560 & 0.000 & 13.786 & 0.361 & 12.857 & 20081027-05:22:00 & \\
\hline 162 & 229.194 & 15.091 & 0.000 & 16.857 & 0.253 & 12.019 & 20081103-07:22:00 & \\
\hline 167 & 276.149 & -9.506 & -44.161 & 18.214 & 0.357 & 6.988 & 20081113-21:52:00 & \\
\hline 172 & 17.888 & -0.560 & 2.795 & 18.357 & 0.299 & 9.783 & 20081124-21:22:00 & \\
\hline 175 & 247.079 & 11.180 & 15.653 & 13.143 & 0.268 & 19.006 & 20081209-02:52:00 & \\
\hline 176 & 70.434 & 5.031 & 50.870 & 16.071 & 0.268 & 10.061 & 20081212-14:22:00 & \\
\hline 179 & 199.004 & 16.211 & -43.603 & 13.500 & 0.262 & 7.826 & 20081227-12:22:00 & \\
\hline 186 & 326.459 & 2.236 & -44.721 & 13.857 & 0.250 & 18.447 & 20090114-12:22:00 & \\
\hline 195 & 346.583 & -6.709 & 2.795 & 12.357 & 0.219 & 10.621 & 20090211-05:22:00 & \\
\hline 198 & 202.360 & 11.180 & -1.118 & 12.929 & 0.296 & 24.595 & 20090218-16:22:00 & \\
\hline 207 & 133.045 & -2.236 & -4.471 & 13.500 & 0.256 & 24.037 & 20090316-18:22:00 & \\
\hline 208 & 220.248 & 11.740 & 40.806 & 12.643 & 0.222 & 11.739 & 20090318-11:52:00 & \\
\hline 213 & 292.918 & -4.471 & -44.721 & 13.286 & 0.234 & 31.025 & 20090326-23:22:00 & \\
\hline 226 & 38.012 & -2.236 & -20.124 & 14.714 & 0.311 & 8.944 & 20090423-08:22:00 & \\
\hline 228 & 195.653 & 2.795 & -39.130 & 16.714 & 0.259 & 13.136 & 20090503-05:22:00 & \\
\hline 234 & 355.529 & -13.415 & 3.913 & 15.286 & 0.287 & 6.149 & 20090522-01:52:00 & \\
\hline 245 & 302.980 & -8.944 & 3.913 & 12.714 & 0.238 & 11.460 & 20090616-21:22:00 & \\
\hline 259 & 226.955 & -6.151 & 0.000 & 13.286 & 0.265 & 13.975 & 20090723-11:52:00 & \\
\hline 262 & 222.480 & -2.795 & 0.000 & 13.429 & 0.287 & 12.019 & 20090804-23:22:00 & \\
\hline 264 & 321.988 & -11.740 & -26.833 & 15.429 & 0.308 & 20.404 & 20090811-22:52:00 & \\
\hline 267 & 176.645 & 5.591 & -44.161 & 12.572 & 0.247 & 13.416 & 20090819-09:08:15 & \\
\hline 288 & 156.521 & -2.795 & -36.335 & 12.072 & 0.404 & 12.857 & 20091031-08:08:15 & \\
\hline 291 & 177.764 & 7.826 & -16.211 & 12.714 & 0.348 & 7.268 & 20091108-11:08:15 & \\
\hline 299 & 168.818 & -2.236 & 5.591 & 13.714 & 0.394 & 13.695 & 20091121-15:08:15 & \\
\hline 307 & 238.136 & 7.267 & -6.151 & 11.714 & 0.314 & 22.361 & 20091216-08:08:15 & \\
\hline 325 & 39.132 & -18.446 & 15.091 & 16.786 & 0.277 & 23.198 & 20100201-21:08:15 & \\
\hline 333 & 219.132 & -15.653 & -22.919 & 11.714 & 0.234 & 16.491 & 20100211-23:08:15 & \\
\hline 337 & 197.888 & 11.180 & -27.392 & 12.214 & 0.330 & 17.888 & 20100214-05:08:15 & \\
\hline 347 & 339.876 & -24.037 & -19.566 & 13.071 & 0.336 & 18.167 & 20100225-00:08:15 & \\
\hline 350 & 144.223 & 36.335 & 1.118 & 11.714 & 0.517 & 28.229 & 20100228-18:08:15 & \\
\hline 351 & 19.008 & -19.008 & 5.031 & 14.714 & 0.397 & 18.167 & 20100301-07:08:15 & \\
\hline 359 & 48.074 & 12.857 & -13.415 & 13.143 & 0.333 & 18.726 & 20100309-11:08:15 & \\
\hline 365 & 19.008 & -8.384 & 2.236 & 13.786 & 0.376 & 11.180 & 20100314-13:08:15 & \\
\hline 369 & 106.211 & -11.740 & -6.709 & 13.714 & 0.385 & 18.726 & 20100319-20:08:15 & \\
\hline 370 & 205.715 & 12.857 & -24.037 & 12.214 & 0.597 & 40.528 & 20100320-09:08:15 & \\
\hline 373 & 21.244 & -1.118 & -39.690 & 15.000 & 0.244 & 9.783 & 20100326-17:08:15 & \\
\hline 376 & 126.335 & 1.678 & 25.715 & 11.857 & 0.388 & 22.640 & 20100329-12:08:15 & \\
\hline 377 & 253.789 & -28.510 & -16.771 & 15.000 & 0.410 & 14.533 & 20100330-14:08:15 & \\
\hline 379 & 258.260 & -26.273 & -1.118 & 13.643 & 0.419 & 16.491 & 20100403-12:08:15 & \\
\hline 383 & 195.653 & -1.678 & 8.383 & 14.928 & 0.367 & 16.211 & 20100408-08:08:15 & \\
\hline 386 & 326.459 & -15.093 & 23.477 & 12.214 & 0.394 & 16.771 & 20100411-08:08:15 & \\
\hline 387 & 266.087 & -25.155 & -29.630 & 13.429 & 0.244 & 9.783 & 20100412-11:08:15 & \\
\hline 389 & 159.876 & 35.217 & -21.803 & 14.786 & 0.499 & 17.888 & 20100413-15:08:15 & \\
\hline 391 & 91.678 & -21.242 & 12.857 & 13.357 & 0.514 & 15.932 & 20100419-04:08:15 & \\
\hline 392 & 86.087 & 26.271 & -27.950 & 15.286 & 0.622 & 10.901 & 20100420-00:08:15 & \\
\hline 404 & 251.554 & -24.597 & 90.000 & 15.500 & 0.665 & 11.460 & 20100506-02:08:15 & \\
\hline 415 & 125.215 & -0.560 & -32.422 & 12.072 & 0.336 & 11.739 & 20100523-00:08:15 & \\
\hline 416 & 320.868 & 7.826 & -9.506 & 12.857 & 0.480 & 13.975 & 20100523-22:08:15 & \\
\hline 418 & 304.099 & -3.913 & -31.304 & 11.929 & 0.520 & 20.123 & 20100524-17:08:15 & \\
\hline 426 & 319.752 & 2.236 & 28.510 & 14.429 & 0.333 & 26.553 & 20100604-16:08:15 & \\
\hline 429 & 336.521 & 30.186 & -40.810 & 16.286 & 0.327 & 6.149 & 20100612-18:08:15 & \\
\hline 432 & 228.074 & -30.186 & -7.267 & 14.571 & 0.351 & 24.317 & 20100615-05:08:15 & \\
\hline 434 & 326.459 & 2.795 & -7.828 & 14.000 & 0.262 & 24.595 & 20100616-21:08:15 & \\
\hline 435 & 98.381 & 20.124 & -23.477 & 13.214 & 0.311 & 18.726 & 20100619-04:08:15 & \\
\hline 437 & 305.219 & 10.622 & 2.236 & 13.714 & 0.213 & 15.652 & 20100621-03:08:15 & \\
\hline 444 & 346.583 & 10.062 & -22.919 & 14.928 & 0.428 & 14.255 & 20100703-08:08:15 & \\
\hline $445 \mathrm{a}$ & 205.715 & -26.273 & 1.118 & 18.072 & 0.284 & 15.373 & 20100703-14:08:15 & \\
\hline $445 b$ & 80.492 & 14.535 & -16.211 & 13.929 & 0.182 & 15.092 & 20100703-14:08:15 & \\
\hline 448 & 320.868 & 40.806 & -40.250 & 12.643 & 0.434 & 13.975 & 20100705-21:08:15 & \\
\hline 449 & 134.161 & -5.031 & -21.242 & 14.000 & 0.268 & 9.783 & 20100706-10:08:15 & \\
\hline 464 & 102.856 & 7.267 & 36.335 & 12.000 & 0.127 & 11.739 & 20100801-06:08:15 & \\
\hline $465 \mathrm{a}$ & 79.380 & 13.415 & -67.640 & 14.000 & 0.729 & 23.198 & 20100801-10:08:15 & \\
\hline $465 \mathrm{~b}$ & 135.281 & 32.981 & -15.653 & 4.857 & 0.271 & 16.211 & 20100801-10:08:15 & \\
\hline 470 & 324.223 & -1.118 & -32.422 & 21.857 & 0.539 & 8.663 & 20100807-21:08:15 & \\
\hline 471 & 200.124 & -22.919 & 2.795 & 12.857 & 0.431 & 23.478 & 20100808-18:08:15 & \\
\hline 474 & 353.293 & -5.031 & 3.353 & 14.357 & 0.816 & 31.025 & 20100814-12:08:15 & \\
\hline
\end{tabular}




\begin{tabular}{|c|c|c|c|c|c|c|c|c|}
\hline No & $\begin{array}{c}\phi \\
{[\mathrm{deg}]}\end{array}$ & $\begin{array}{c}\theta \\
{[\mathrm{deg}]}\end{array}$ & $\begin{array}{c}\gamma \\
{[\mathrm{deg}]}\end{array}$ & $\begin{array}{c}\mathrm{h}_{\text {front }} \\
\mathrm{r}_{\odot} \\
\end{array}$ & $\begin{array}{l}\kappa \\
-\end{array}$ & $\begin{array}{c}\alpha \\
{[\mathrm{deg}]}\end{array}$ & $\begin{array}{l}\text { Time stamp } \\
\text { y-m-d h-m-s }\end{array}$ & $\begin{array}{r}\text { Flag } \\
-\end{array}$ \\
\hline 476 & 310.806 & 32.981 & -25.155 & 15.214 & 0.373 & 21.522 & 20100815-15:08:15 & \\
\hline 479 & 348.818 & -6.709 & -43.043 & 16.071 & 0.717 & 12.019 & 20100818-07:08:15 & \\
\hline 500 & 264.967 & 22.360 & 2.795 & 13.214 & 0.579 & 19.565 & 20100911-07:08:15 & \\
\hline 511 & 176.645 & 42.484 & -1.118 & 14.714 & 0.299 & 22.919 & 20101001-05:08:15 & \\
\hline 515 & 280.620 & 21.802 & -4.471 & 9.429 & 0.345 & 20.404 & 20101006-10:08:15 & \\
\hline 516 & 8.942 & 45.279 & 23.477 & 13.214 & 0.357 & 37.174 & 20101007-15:08:15 & \\
\hline 519 & 225.839 & 6.709 & -41.366 & 13.571 & 0.523 & 35.496 & 20101011-06:08:15 & \\
\hline 525 & 69.318 & -25.715 & -40.810 & 13.357 & 0.545 & 11.739 & 20101026-14:08:15 & \\
\hline 528 & 205.715 & -17.888 & 2.795 & 10.572 & 0.487 & 35.217 & 20101028-15:08:15 & \\
\hline 546 & 86.087 & -25.715 & -2.795 & 12.857 & 0.382 & 19.286 & 20101116-15:08:15 & \\
\hline 551 & 22.360 & -21.803 & 22.919 & 13.786 & 0.397 & 22.361 & 20101124-09:08:15 & \\
\hline 552 & 157.640 & -30.746 & 29.068 & 14.214 & 0.305 & 5.870 & 20101125-23:08:15 & \\
\hline 561 & 163.231 & -34.659 & 45.839 & 15.214 & 0.407 & 16.491 & 20101207-00:08:15 & \\
\hline $566 \mathrm{a}$ & 214.657 & -13.975 & 11.180 & 20.500 & 0.404 & 24.875 & 20101212-09:08:15 & \\
\hline $566 \mathrm{~b}$ & 88.322 & 33.539 & -54.223 & 19.143 & 0.164 & 3.354 & 20101212-11:08:15 & \\
\hline $568 \mathrm{a}$ & 172.174 & 29.628 & -36.895 & 13.143 & 0.382 & 12.578 & 20101214-18:08:15 & \\
\hline $568 \mathrm{~b}$ & 169.938 & 50.310 & -40.250 & 13.643 & 0.357 & 13.695 & 20101214-18:08:15 & \\
\hline 574 & 349.938 & -13.975 & -15.653 & 13.357 & 0.462 & 10.341 & 20101223-12:08:15 & \\
\hline 576 & 180.000 & -29.068 & 6.709 & 12.786 & 0.613 & 15.373 & 20101226-09:08:15 & \\
\hline 587 & 200.124 & 21.240 & -35.217 & 16.571 & 0.262 & 8.385 & 20110110-06:08:15 & \\
\hline 590 & 349.938 & -16.211 & 2.236 & 15.857 & 0.339 & 58.416 & 20110112-15:08:15 & \\
\hline 597 & 121.864 & -21.803 & -32.981 & 17.500 & 0.290 & 7.268 & 20110120-23:08:15 & \\
\hline 603 & 4.471 & -8.384 & -39.690 & 23.357 & 0.148 & 6.988 & 20110124-10:08:15 & \\
\hline 606 & 272.790 & 29.628 & 0.000 & 11.143 & 0.364 & 25.714 & 20110126-05:08:15 & \\
\hline 608 & 48.074 & 44.161 & -74.347 & 10.643 & 0.407 & 36.895 & 20110128-05:08:15 & \\
\hline 612 & 182.236 & -12.298 & -20.124 & 17.000 & 0.262 & 11.739 & 20110130-20:08:15 & \\
\hline 614 & 0.000 & 3.913 & 0.000 & 13.500 & 0.425 & 33.540 & 20110202-02:08:15 & \\
\hline 615 & 0.000 & 21.802 & 6.709 & 11.428 & 0.364 & 16.491 & 20110202-07:08:15 & \\
\hline 620 & 237.020 & -11.180 & 7.267 & 13.786 & 0.259 & 21.522 & 20110210-11:08:15 & \\
\hline 622 & 0.000 & 6.709 & 12.298 & 14.571 & 0.277 & 23.198 & 20110215-00:08:15 & \\
\hline 623 & 8.942 & -6.151 & -36.895 & 12.929 & 0.330 & 28.509 & 20110215-04:08:15 & \\
\hline 624 & 339.876 & 31.304 & 29.628 & 13.357 & 0.388 & 20.683 & 20110216-08:08:15 & \\
\hline 628 & 141.984 & -51.988 & -20.124 & 14.357 & 0.477 & 35.217 & 20110224-15:08:15 & \\
\hline 631 & 86.087 & 25.715 & -22.919 & 15.929 & 0.413 & 10.901 & 20110226-23:08:15 & \\
\hline 635 & 159.876 & -22.360 & -19.566 & 13.214 & 0.299 & 12.857 & 20110303-10:08:15 & \\
\hline 646 & 32.422 & 39.690 & -5.031 & 16.000 & 0.951 & 55.901 & 20110307-21:08:15 & \\
\hline 647 & 15.653 & 3.913 & 0.000 & 18.286 & 0.800 & 11.739 & 20110308-07:08:15 & $\mathrm{h}$ \\
\hline 648 & 0.000 & -43.603 & 14.535 & 13.786 & 0.557 & 67.081 & 20110309-00:08:15 & \\
\hline 651 & 177.764 & -17.330 & 8.384 & 14.357 & 0.321 & 20.404 & 20110312-06:08:15 & $\mathrm{n}$ \\
\hline 653 & 335.401 & 36.895 & -35.777 & 16.928 & 0.256 & 51.988 & 20110314-15:08:15 & \\
\hline 655 & 36.893 & 43.603 & -39.690 & 14.643 & 0.723 & 14.814 & 20110316-23:08:15 & \\
\hline 656 & 122.980 & -23.477 & -23.477 & 15.071 & 0.530 & 23.198 & 20110317-15:08:15 & \\
\hline 661 & 51.426 & 22.360 & -68.758 & 16.143 & 0.330 & 63.167 & 20110321-04:08:15 & $\mathrm{d}$ \\
\hline 665 & 78.260 & 35.217 & -5.591 & 11.786 & 0.302 & 21.802 & 20110324-00:08:15 & \\
\hline 667 & 61.492 & 19.566 & 0.000 & 11.714 & 0.453 & 19.286 & 20110324-15:08:15 & \\
\hline 668 & 234.781 & -6.709 & 0.000 & 12.572 & 0.385 & 24.037 & 20110325-18:08:15 & \\
\hline 669 & 30.182 & 15.091 & 0.000 & 11.643 & 0.404 & 26.553 & 20110326-09:08:15 & \\
\hline 673 & 0.000 & 48.074 & 29.628 & 12.714 & 0.287 & 23.478 & 20110328-20:08:15 & \\
\hline 675 & 76.025 & -2.236 & 0.000 & 16.786 & 0.825 & 20.123 & 20110329-22:08:15 & $\mathrm{h}$ \\
\hline 679 & 86.087 & -47.516 & 21.802 & 12.786 & 0.388 & 34.379 & 20110403-06:08:15 & $\mathrm{n}$ \\
\hline 680 & 86.087 & 7.826 & -16.771 & 12.857 & 0.262 & 19.565 & 20110404-06:08:15 & \\
\hline 681 & 237.020 & 31.304 & 20.124 & 15.143 & 0.336 & 16.771 & 20110404-11:08:15 & $\mathrm{c}$ \\
\hline 682 & 58.133 & -17.330 & 0.000 & 13.429 & 0.588 & 50.870 & 20110407-12:08:15 & \\
\hline 683 & 190.062 & -36.335 & -30.746 & 13.071 & 0.530 & 15.373 & 20110407-14:08:15 & \\
\hline 684 & 195.653 & 3.913 & 29.628 & 15.000 & 0.336 & 22.919 & 20110408-03:08:15 & \\
\hline 689 & 25.711 & 30.746 & -3.913 & 12.143 & 0.619 & 38.571 & 20110412-06:08:15 & d \\
\hline 696 & 240.372 & 21.802 & 16.211 & 15.857 & 0.213 & 10.901 & 20110417-10:08:15 & \\
\hline 697 & 127.454 & 6.149 & 7.267 & 12.500 & 0.382 & 25.714 & 20110417-17:08:15 & \\
\hline 703 & 358.880 & 25.155 & -27.392 & 13.286 & 0.311 & 17.888 & 20110425-02:08:15 & \\
\hline 706 & 0.000 & 40.808 & -24.037 & 13.143 & 0.487 & 19.565 & 20110427-11:08:15 & \\
\hline 711 & 84.967 & 31.304 & 15.653 & 12.286 & 0.490 & 25.994 & 20110501-23:08:15 & \\
\hline 716 & 306.331 & 4.471 & 0.000 & 17.572 & 0.665 & 50.870 & 20110506-12:08:15 & $\mathrm{hn}$ \\
\hline 723 & 101.740 & -30.746 & -22.360 & 13.929 & 0.342 & 17.888 & 20110513-02:08:15 & \\
\hline 732 & 316.397 & -1.678 & -16.211 & 14.786 & 0.760 & 43.323 & 20110518-21:08:15 & $\mathrm{hn}$ \\
\hline 733 & 298.508 & 21.802 & 10.062 & 21.857 & 0.151 & 15.092 & 20110519-09:08:15 & \\
\hline 735 & 23.479 & 21.240 & 0.000 & 12.214 & 0.333 & 25.714 & 20110521-02:08:15 & \\
\hline
\end{tabular}




\begin{tabular}{|c|c|c|c|c|c|c|c|c|}
\hline No & $\begin{array}{c}\phi \\
{[\mathrm{deg}]}\end{array}$ & $\begin{array}{c}\theta \\
{[\mathrm{deg}]}\end{array}$ & $\begin{array}{c}\gamma \\
{[\mathrm{deg}]}\end{array}$ & $\begin{array}{c}\mathrm{h}_{\text {front }} \\
\mathrm{r}_{\odot} \\
\end{array}$ & $\begin{array}{l}\kappa \\
- \\
\end{array}$ & $\begin{array}{c}\alpha \\
{[\mathrm{deg}]}\end{array}$ & $\begin{array}{l}\text { Time stamp } \\
\mathrm{y}-\mathrm{m}-\mathrm{d} \text { h-m-s }\end{array}$ & $\begin{array}{r}\text { Flag } \\
- \\
\end{array}$ \\
\hline 743 & 34.654 & -6.709 & 13.415 & 17.214 & 0.634 & 53.105 & 20110529-15:08:15 & \\
\hline 748 & 41.364 & -13.975 & 1.118 & 12.857 & 0.182 & 15.373 & 20110601-11:08:15 & \\
\hline 750 & 60.368 & -4.471 & 5.031 & 12.929 & 0.431 & 23.758 & 20110601-22:08:15 & \\
\hline 751 & 34.654 & -7.828 & 36.895 & 15.071 & 0.253 & 23.198 & 20110602-10:08:15 & \\
\hline 753 & 27.950 & -18.446 & 8.944 & 13.571 & 0.302 & 20.404 & 20110604-00:08:15 & $\mathrm{n}$ \\
\hline 754 & 139.752 & 6.149 & -24.037 & 16.500 & 0.705 & 21.242 & 20110604-08:08:15 & \\
\hline 755 & 140.868 & 16.211 & -44.161 & 21.286 & 0.914 & 36.615 & 20110604-23:08:15 & \\
\hline 756 & 335.401 & 47.516 & 79.938 & 14.143 & 0.376 & 33.540 & 20110605-11:08:15 & \\
\hline 757 & 97.268 & -32.422 & 32.420 & 13.357 & 0.370 & 11.460 & 20110606-13:08:15 & \\
\hline 758 & 33.541 & -13.975 & -5.031 & 13.071 & 0.600 & 10.341 & 20110607-08:08:15 & \\
\hline 760 & 165.467 & 34.099 & -25.715 & 12.072 & 0.253 & 12.578 & 20110608-14:08:15 & \\
\hline 762 & 278.384 & 57.019 & 54.223 & 13.143 & 0.530 & 27.950 & 20110609-17:08:15 & \\
\hline 769 & 355.529 & 7.826 & -44.161 & 19.500 & 0.702 & 31.584 & 20110613-00:08:15 & hn \\
\hline 770 & 27.950 & -7.828 & 77.141 & 16.286 & 0.216 & 21.802 & 20110613-06:08:15 & $\mathrm{d}$ \\
\hline 772 & 202.360 & -0.560 & 36.335 & 16.928 & 0.284 & 57.299 & 20110614-12:08:15 & $\mathrm{n}$ \\
\hline 776 & 0.000 & 2.795 & -4.471 & 14.071 & 0.247 & 22.081 & 20110620-22:08:15 & \\
\hline 777 & 148.694 & 12.857 & -8.944 & 13.786 & 0.317 & 26.553 & 20110621-05:08:15 & $\mathrm{n}$ \\
\hline 789 & 111.802 & -38.012 & -24.037 & 10.643 & 0.474 & 5.590 & 20110705-02:08:15 & \\
\hline 794 & 277.268 & -14.535 & 1.678 & 14.071 & 0.431 & 23.198 & 20110709-03:08:15 & $\mathrm{d}$ \\
\hline 796 & 100.620 & 30.186 & -12.857 & 12.714 & 0.588 & 43.323 & 20110710-01:08:15 & $\mathrm{m}$ \\
\hline 797 & 196.765 & 41.364 & 1.118 & 15.071 & 0.308 & 17.888 & 20110710-17:08:15 & $\mathrm{n}$ \\
\hline 809 & 332.050 & 36.335 & -22.919 & 15.071 & 0.345 & 13.136 & 20110720-21:08:15 & \\
\hline 810 & 334.282 & -30.746 & -24.597 & 12.214 & 0.453 & 23.478 & 20110722-01:08:15 & \\
\hline 813 & 240.372 & -7.828 & -40.810 & 14.357 & 0.588 & 25.993 & 20110725-21:08:15 & d \\
\hline 817 & 177.764 & -32.422 & -15.653 & 13.929 & 0.462 & 14.814 & 20110729-01:08:15 & \\
\hline 821 & 324.223 & 24.037 & -40.250 & 12.572 & 0.520 & 19.845 & 20110803-15:08:15 & \\
\hline 822 & 328.691 & 21.802 & 60.372 & 13.786 & 0.794 & 62.888 & 20110804-05:08:15 & \\
\hline 823 & 119.628 & 30.186 & -6.151 & 14.429 & 0.351 & 10.061 & 20110804-11:08:15 & \\
\hline 831 & 282.852 & 2.236 & 19.566 & 16.571 & 0.782 & 27.392 & 20110811-13:08:15 & \\
\hline 834 & 320.868 & 19.006 & 40.246 & 15.429 & 0.373 & 10.621 & 20110813-15:08:15 & \\
\hline 841 & 209.070 & -13.975 & 0.000 & 13.214 & 0.456 & 25.155 & 20110824-04:08:15 & cnd \\
\hline 844 & 143.107 & 29.628 & 12.298 & 14.571 & 0.487 & 9.783 & 20110828-00:08:15 & \\
\hline 845 & 120.740 & 46.957 & -39.690 & 13.500 & 0.397 & 39.410 & 20110828-11:08:15 & \\
\hline 851 & 127.454 & -32.981 & 23.477 & 13.857 & 0.563 & 9.503 & 20110902-17:08:15 & \\
\hline 861 & 245.963 & 34.099 & 26.833 & 14.928 & 0.416 & 35.496 & 20110907-01:08:15 & \\
\hline 867 & 226.955 & 31.864 & 3.353 & 13.929 & 0.450 & 15.932 & 20110908-02:08:15 & \\
\hline 870 & 0.000 & 54.223 & -90.000 & 11.357 & 0.357 & 44.441 & 20110909-00:08:15 & \\
\hline $871 \mathrm{a}$ & 201.244 & -2.795 & 15.653 & 13.429 & 0.370 & 19.286 & 20110909-13:08:15 & $\mathrm{n}$ \\
\hline $871 b$ & 233.665 & 56.459 & -35.217 & 11.000 & 0.247 & 11.739 & 20110909-13:08:15 & \\
\hline 873 & 178.880 & 47.516 & -39.690 & 14.500 & 0.480 & 10.901 & 20110910-07:08:15 & \\
\hline 874 & 192.298 & -16.211 & 25.715 & 13.643 & 0.428 & 34.938 & 20110910-13:08:15 & \\
\hline 879 & 131.926 & 20.684 & -5.591 & 12.929 & 0.465 & 12.299 & 20110914-03:08:15 & $\mathrm{n}$ \\
\hline 881 & 130.806 & -6.151 & 29.068 & 14.357 & 0.296 & 12.578 & 20110915-03:08:15 & \\
\hline 890 & 119.628 & 4.471 & 15.653 & 18.429 & 0.533 & 18.726 & 20110922-00:08:15 & $\mathrm{h}$ \\
\hline 891 & 280.620 & 3.353 & -24.037 & 20.214 & 0.659 & 18.726 & 20110922-12:08:15 & \\
\hline 897 & 278.384 & 5.591 & -54.783 & 16.928 & 0.720 & 26.833 & 20110924-14:08:15 & \\
\hline 898 & 289.566 & 32.420 & -12.298 & 15.143 & 0.250 & 21.242 & 20110924-21:08:15 & \\
\hline 901 & 33.541 & -25.155 & 22.919 & 15.571 & 0.357 & 6.428 & 20110925-12:08:15 & $\mathrm{m}$ \\
\hline $906 \mathrm{a}$ & 111.802 & 24.597 & 16.771 & 14.500 & 0.385 & 9.783 & 20110929-23:08:15 & \\
\hline $906 \mathrm{~b}$ & 53.665 & -38.572 & 46.395 & 15.500 & 0.216 & 32.143 & 20110929-23:08:15 & \\
\hline 907 & 67.082 & 45.839 & 31.304 & 14.928 & 0.548 & 14.814 & 20110930-07:08:15 & $\mathrm{c}$ \\
\hline 911 & 136.393 & 8.383 & 21.240 & 20.286 & 0.665 & 38.292 & 20111001-23:08:15 & \\
\hline 919 & 153.169 & -30.746 & -8.384 & 14.000 & 0.317 & 8.385 & 20111005-23:08:15 & \\
\hline 923 & 304.099 & 12.857 & 0.000 & 13.429 & 0.447 & 23.758 & 20111010-11:08:15 & $\mathrm{n}$ \\
\hline 928 & 308.570 & 3.353 & 79.378 & 19.286 & 0.462 & 24.037 & 20111014-15:08:15 & \\
\hline 941 & 59.256 & 44.721 & -6.151 & 16.571 & 0.585 & 45.839 & 20111022-13:08:15 & \\
\hline 950 & 306.331 & 10.062 & 15.091 & 13.643 & 0.490 & 10.341 & 20111026-15:08:15 & \\
\hline 957 & 43.603 & -23.477 & -1.678 & 14.500 & 0.394 & 10.901 & 20111029-01:08:15 & \\
\hline 962 & 206.831 & 30.746 & 3.913 & 14.214 & 0.317 & 15.652 & 20111031-21:08:15 & $\mathrm{n}$ \\
\hline 967 & 360.000 & -13.415 & -46.957 & 11.214 & 0.631 & 31.584 & 20111104-00:08:15 & \\
\hline 968 & 54.781 & 6.709 & -79.940 & 18.286 & 0.689 & 55.062 & 20111104-04:08:15 & $\mathrm{h}$ \\
\hline 969 & 199.004 & 55.901 & -57.578 & 13.286 & 0.425 & 15.932 & 20111105-10:08:15 & \\
\hline 971 & 130.806 & 38.012 & 10.062 & 12.929 & 0.391 & 13.975 & 20111108-03:08:15 & \\
\hline 974 & 34.654 & -27.950 & -8.944 & 14.429 & 0.437 & 26.833 & 20111109-13:08:15 & $\mathrm{n}$ \\
\hline 975 & 60.368 & 19.006 & 13.975 & 14.571 & 0.880 & 35.777 & 20111109-15:08:15 & \\
\hline 980 & 318.632 & 8.383 & 44.721 & 19.429 & 0.729 & 22.640 & 20111112-23:08:15 & $\mathrm{h}$ \\
\hline
\end{tabular}




\begin{tabular}{rrrrrrrrr}
\hline No & \multicolumn{1}{c}{$\phi$} & \multicolumn{1}{c}{$\theta$} & $\begin{array}{c}\gamma \\
{[\mathrm{deg}]}\end{array}$ & \multicolumn{1}{c}{$\begin{array}{c}\mathrm{h} \text { front } \\
{[\mathrm{deg}]}\end{array}$} & $\begin{array}{c}c \\
\mathrm{r}_{\odot}\end{array}$ & \multicolumn{1}{c}{$\begin{array}{c}\alpha \\
{[\mathrm{deg}]}\end{array}$} & $\begin{array}{c}\text { Time stamp } \\
\text { y-m-d h-m-s }\end{array}$ & $\begin{array}{r}\text { Flag } \\
-\end{array}$ \\
\hline 981 & 197.888 & 12.298 & -63.727 & 15.571 & 0.416 & 25.994 & $20111113-21: 08: 15$ & $\mathrm{~d}$ \\
983 & 41.364 & -34.099 & -4.471 & 14.143 & 0.483 & 9.783 & $20111115-00: 08: 15$ & $\mathrm{~d}$ \\
985 & 215.777 & 5.591 & 69.316 & 12.072 & 0.333 & 34.098 & $20111115-08: 08: 15$ & \\
1002 & 354.409 & 25.715 & -72.112 & 16.214 & 0.382 & 10.341 & $20111123-01: 08: 15$ & \\
1007 & 282.852 & 13.975 & -41.366 & 14.429 & 0.634 & 44.161 & $20111126-09: 08: 15$ & \\
1013 & 53.665 & -32.422 & -25.155 & 15.357 & 0.351 & 24.037 & $20111130-02: 08: 15$ & \\
1017 & 210.182 & 58.696 & 77.701 & 13.929 & 0.517 & 8.663 & $20111203-01: 08: 15$ & \\
1024 & 247.079 & 26.271 & 64.845 & 14.071 & 0.311 & 19.565 & $20111207-09: 08: 15$ & \\
1050 & 103.975 & -17.888 & 39.130 & 11.714 & 0.425 & 25.994 & $20111221-05: 08: 15$ & \\
1053 & 20.120 & 15.091 & 43.043 & 14.071 & 0.376 & 15.652 & $20111223-09: 08: 15$ & $\mathrm{n}$ \\
1058 & 195.653 & 21.240 & 34.099 & 15.786 & 0.277 & 17.888 & $20111225-05: 08: 15$ & \\
1061 & 188.942 & 22.919 & 36.895 & 12.286 & 0.364 & 15.932 & $20111226-14: 08: 15$ & \\
1067 & 133.045 & 40.246 & 41.926 & 14.000 & 0.238 & 21.802 & $20111229-11: 08: 15$ & \\
1068 & 243.727 & 7.267 & -36.335 & 15.286 & 0.837 & 17.888 & $20111229-19: 08: 15$ & $\mathrm{hn}$ \\
\hline
\end{tabular}

\section{A.6 The CME Online Database}

As mentioned in this thesis an online CME database was developed during the AFFECTS project time and financed by this project. The database contains the introduced CME lists, namely the 'Overall CME list', the 'Best-of CME list' and the 'GCS modeling results list' which provides the numerical results. Furthermore coronagraph images of each CME from the 'GCS results list' are provided together with images which show the CME's GCS fit. In addition a download link to the STEREO/SECCHI/COR2 synoptic movie for each CMEs is presented. This allows to view the CME propagation in the COR2 field of view in a video clip. With this the database provides a very practicable access and overview to all analysed CME events via a website ${ }^{32}$. Figure 84 presents the images of a GCS modelled CME from the 'GCS modeling results list'. The website presents a brief introduction to the CME database and in which scientific context and project framework it has been developed. Links to other CME catalogues and involved scientists are mentioned as well as information to further reading. The database section comprises all event lists. Each entry of an CME event in the 'GCS modeling Results list' links to a new webpage with the graphical material as shown in Figure 84 and with additional detailed information. In order to keep the tables user-friendly the columns can be (de)activated to get a clear presentation of the desired data. Filter functions allow to select only events of a certain range in time or for a defined range in speed.

The CME database and website was developed and implemented by Sabyasachi Gosh and Julius Achenbach and is online for public access since 2014, www . affects-fp7. eu/cme-database. 


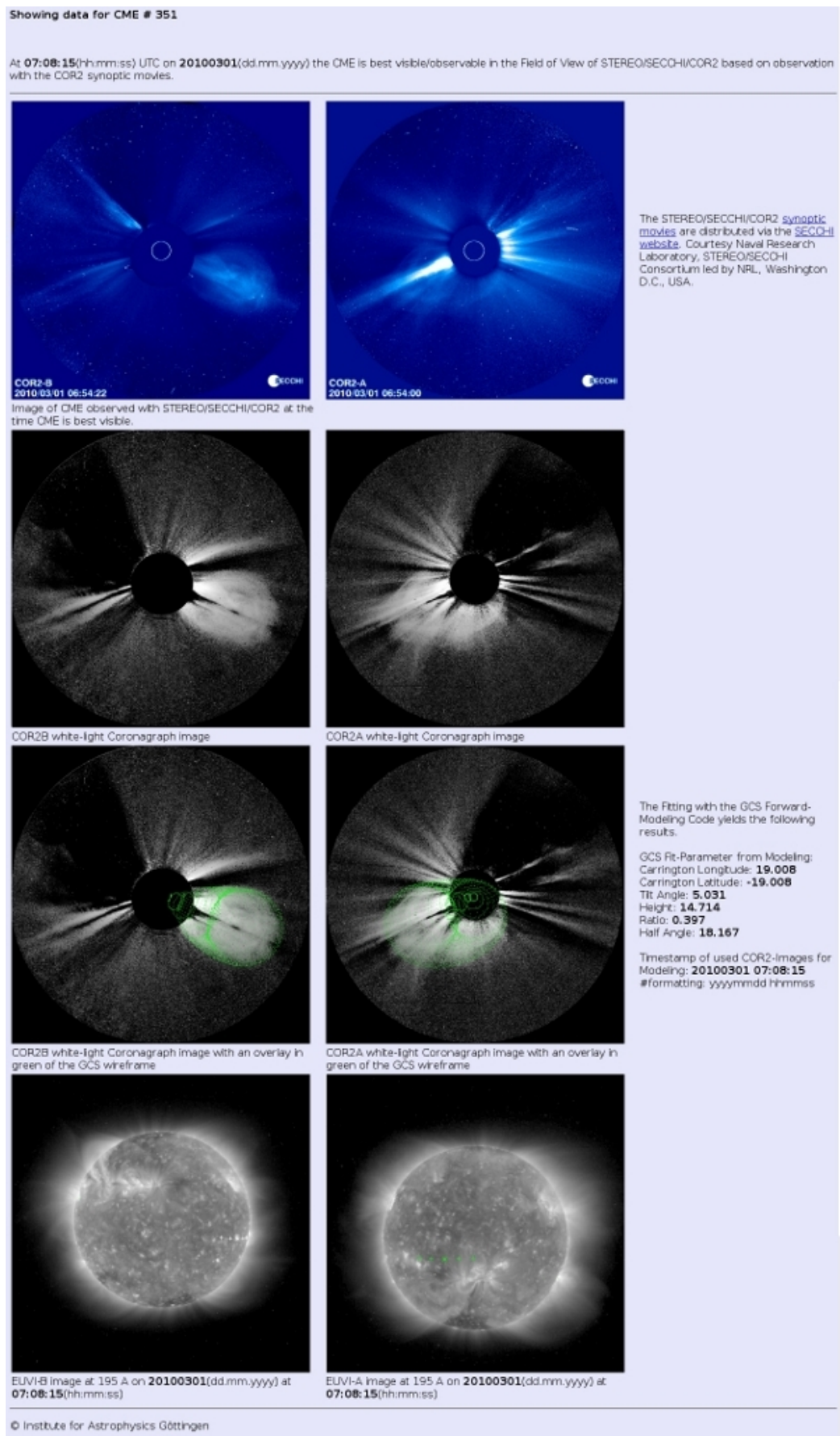

Fig. 84: An overview is provided for each CME of the 'GCS modeling results' list. Top row: Coronagraph images and the corresponding COR2 synoptic movie (Download link) show the CME. Second row: The same with IDL processed COR2 white-light coronagraph images without and (third row) with an overlay of the GCS fit (green). This fit corresponds to the numerical GCS results of the presented CME. The results of the fit are listed right hand to the images. Finally the EUVI images present the GCS footpoint line for comparison with possible source region indicators. 


\section{A.7 Acronyms}

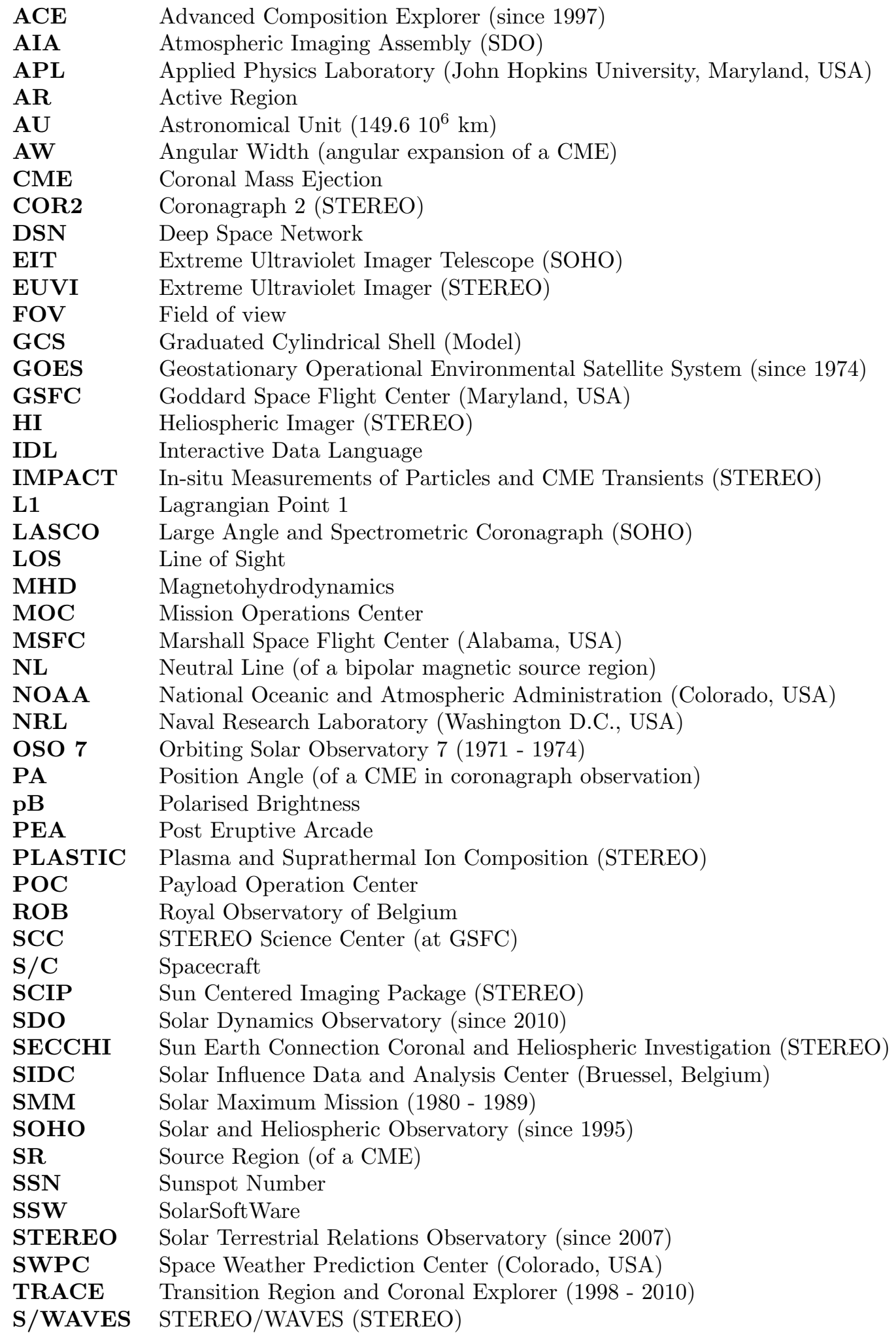




\section{B References}

[1] Aschwanden, J.M. (2006): Physics of the Solar Corona - An Introduction with Problems and Solutions, Springer, Berlin.

[2] Belvedere, G., Proctor, M.R.E. and Lanzafame, G. (1991): The latitude belts of solar activity as a consequence of a boundary-layer dynamo, Letters of Nature, 350, 481-483.

[3] Billings, D.E. (1966): A Guide to the Solar Corona, Academic Press, New York.

[4] Bohlin, J.D. and Garrison, L.M. (1974): Numerical calculation of Thomson scattering from inhomogeneous models of the corona, and application to streamers of the 1970 and 1972 eclipses, Solar Physics., 38, 165-179.

[5] Bosman, E., Bothmer, V., Nistico, G., Vourlidas, A., Howard, R.A. and Davies, J.A. (2012): Three-Dimensional Properties of Coronal Mass Ejections from STEREO/SECCHI Observations, Solar Physics, 281, 167-185.

[6] Bothmer, V. and Schwenn, R. (1997): The structure and origin of magnetic clouds in the solar wind, Annales Geophysicae, 16, 1-24.

[7] Bothmer, V. (2006): The solar atmosphere and space weather, Solar System Update, 1-53.

[8] Bothmer, V. and Daglis, I.A. (2007): Space Weather - Physics and Effects, Springer Praxis Publishing.

[9] Bothmer, V. (2010): SOTERIA-WP3 Report - Deliverables 3.2, http://soteria-space.eu/ doc/reports/SOTERIA_D3_2.pdf.

[10] Bougeret, J.L., Goetz, K., Kaiser, M.L., Bale, S.D., Kellogg, P.J., Maksimovic, M., Monge, N., Monson, S.J., Astier, P.L., Davy, S., Dekkali, M., Hinze, J.J., Manning, R.E., AguilarRodriguez, E., Bonnin, X., Briand, C., Cairns, I.H., Cattell, C.A., Cecconi, B., Eastwood, J., Ergun, R.E., Fainberg, J., Hoang, S., Huttunen, K.E.J., Krucker, S., Lecacheux, A., MacDowall, R.J., Macher, W., Mangeney, A., Meetre, C.A., Moussas, X., Nguyen, Q.N., Oswald, T.H., Pulupa, M., Reiner, M.J., Robinson, P.A., Rucker, H., Salem, C., Santolik, O., Silvis, J.M., Ullrich, R., Zarka, P. and Zouganelis, I. (2008): S/WAVES: The Radio and Plasma Wave Investigation on the STEREO Mission, Space Science Reviews, 136, 487-528.

[11] Brueckner, G.E., Howard, R.A., Koomen, M.J., Korendyke, C.M., Michels, D.J., Moses, J.D., Socker, D.G., Dere, K.P., Lamy, P.L., Llebaria, A., Bout, M.V., Schwenn, R., Simnett, G.M., Bedford, D.K. and Eyles, C.J. (1995): The Large Angle Spectroscopic Coronagraph (LASCO) - Visible light coronal imaging and Spectroscopy, Solar Physics, 162, 357-402.

[12] Cargill, P.J. (2004): On the aerodynamic Drag force acting on interplanetary coronal mass ejections, Solar Physics, 221, 135-149.

[13] Chen, J. and Garren, D.A. (1993): Interplanetary magnetic clouds: topology and driving mechanism, Geophysical Research Letters, 20, 2319-2322.

[14] Chen, J. (1996): Theory of prominence eruption and propagation: interplanetary consequences, Journal of Geophysical Research, 101, 27499-27520.

[15] Chen, J., Howard, R.A., Brueckner, G.E., Santoro, R., Krall, J., Paswaters, S.E., St. Cyr, O.C., Schwenn, R., Lamy, P., and Simnett, G. M. (1997): Evidence of an erupting magnetic flux rope: LASCO Coronal Mass Ejection of 1997 April 13, The Astrophysical Journal, 490, 191194. 
[16] Chen, J., Santoro, R.A., Krall, J., Howard, R.A., Duffin, R., Moses, J.D., Brueckner, G.E., Darnell, J.A. and Burkepile, J.T. (2000): Magnetic geometry and dynamics of the fast coronal mass ejection of 1997 September 9, The Astrophysical Journal, 533, 481-500.

[17] Chen, P.F. (2011): Coronal Mass Ejections: Models and Their Observational Basis, Living Reviews Solar Physics, 8, 1. URL http://www.livingreviews.org/Irsp-2011-1 (cited on 18.12.2015).

[18] Cremades, H. and Bothmer, V. (2004): On the three-dimensional configuration of coronal mass ejections, Astronomy \& Astrophysics, 422, 307-322.

[19] Cremades, H. and Bothmer, V. (2005): Geometrical properties of coronal mass ejections, Proceedings IAU Symposium, 226, 48-54.

[20] Cremades, H. and St. Cyr, O.C. (2007): Coronal mass ejections: Solar cycle aspects, Advances in Space Research, 40, 1042-1048.

[21] Crifo, F., Picat, J.P. and Cailloux, M. (1983): Coronal transients-loop or bubble, Solar Physics, 83, 143-152.

[22] Davies, J.A., Harrison, R.A., Perry, C.H., Moestl, C., Lugaz, N., Rollett, T., Davis, C.J., Crothers, S.R., Temmer, M., Eyles, C.J. and Savani, N.P. (2012): A self-similar expansion model for use in solar wind transient propagation studies, The Astrophysical Journal, $\mathbf{7 5 0}$, 23.

[23] Davis, C.J., Davies, J.A., Lockwood, M., Rouillard, A.P., Eyles, C.J. and Harrison, R.A. (2009): Stereoscopic imaging of an Earth-impacting solar coronal mass ejection: A major milestone for the STEREO mission, Geophysical Research Letters, 36, L08102.

[24] De Koning, C.A., Pizzo, V.J. and Biesecker, D.A. (2009): Calculating CME velocity in nearreal-time using geometric and polarimetric techniques, AAS/Solar Physics Division Meeting, 41,841 .

[25] Delaboudiniere, J.-P., Artzner, G.E., Brunaud, J., Gabriel, A.H., Hochedez, J.F., Millier, F., Song, X.Y., Au, B., Dere, K.P., Howard, R.A., Kreplin, R., Michels, D.J., Moses, J.D., Defise, J.M., Jamar, C., Rochus, P., Chauvineau, J.P., Marioge, J.P., Catura, R.C., Lemen, J.R., Shing, L., Stern, R.A., Gurman, J.B., Neupert, W.M., Maucherat, A., Clette, F., Cugnon, P. and Van Dessel, E.L. (1995): EIT: Extreme-Ultraviolet Imaging Telescope for the SOHO Mission, Solar Physics, 162, 291-312.

[26] Demoulin, P. and Priest, E.R. (1989): How to form a dip in a magnetic field before the formation of a solar prominence, Hvar Observatory Bulletin, 13, 261-268.

[27] Domingo, V., Fleck, B. and Poland, A.I. (1995): The SOHO Mission: An overview, Solar Physics, 162, 1-37.

[28] Eddy, J.A. (1974): A Nineteenth-century Coronal Transient, Astronomy and Astrophysics, 34, 235-240.

[29] Einaudi, G. and Van Hoven, G. (1983): The stability of coronal loops: Finite-Length and pressure-profile limits*, Solar Physics, 88, 163-177.

[30] ESA SSA Team (2013): Space Situational Awareness - Space Weather System Requirements Document, $S S A-S W E-R S-R D-0001,2013-07-09$, http://ssa-be-vm-fe-09p.ssa.esa.int/ DOCS/SSA-SWE/SSA-SWE-RS-RD-0001_i1r4.pdf.

[31] Fisher, R.R. and Munro, R.H. (1984): Coronal transient geometry. The flare-associated event of 1981 March 25, The Astrophysical Journal, 280, 428.

[32] Forbes, T.G. (2000): A review on the genesis of coronal mass ejections, Journal of Geophysical Research, 105, 23153-23166. 
[33] Frazin, R.A. and Janzen, P. (2002): Tomography of the solar corona. II. robust, regularized, positive estimation of the three-dimensional electron density distribution from LASCO-C2 polarized white-light images, The Astrophysical Journal, 570, 408-422.

[34] Galvin, A.B., Kistler, L.M., Popecki, M.A., Farrugia, C.J., Simunac, K.D.C., Ellis, L., Möbius, E., Lee, M.A., Boehm, M., Carroll, J., Crawshaw, A., Conti, M., Demaine, P., Ellis, S., Gaidos, J.A., Googins, J., Granoff, M., Gustafson, A., Heirtzler, D., King, B., Knauss, U., Levasseur, J., Longworth, S., Singer, K., Turco, S., Vachon, P., Vosbury, M., Widholm, M., Blush, L.M., Karrer, R., Bochsler, P., Daoudi, H., Etter, A., Fischer, J., Jost, J., Opitz, A., Sigrist, M., Wurz, P., Klecker, B., Ertl, M., Seidenschwang, E., Wimmer-Schweingruber, R.F., Koeten, M., Thompson, B. and Steinfeld, D. (2008): The Plasma and Suprathermal Ion Composition (PLASTIC) Investigation on the STEREO Observatories, Space Science Reviews, 136, 437-486.

[35] Gibson, S.E. and Fan, Y. (2006): Coronal prominence structure and dynamics: A magnetic flux rope interpretation, Journal of Geophysical Research, 111, A12103, doi:10.1029/2006JA011871.

[36] Golub, L. and Pasachoff, J. (2010): The Solar Corona, Cambridge University Press.

[37] Gopalswamy, N., Lara, A., Yashiro, S. and Howard, R.A. (2003): Coronal Mass Ejections and solar polarity reversal, The Astrophysical Journal, 598, 63-66.

[38] Gopalswamy, N., Lara, A., Yashiro, S., Nunes, S. and Howard, R.A. (2003): Coronal mass ejection activity during solar cycle 23, Proceedings ISCS 2003 Symposium, ESA SP-535, 403-414.

[39] Gopalswamy, N. (2006): Coronal Mass Ejections of Solar Cycle 23, Journal of Astrophysics and Astronomy, 27, 243-254.

[40] Gopalswamy, N., Yashiro, S., Michalek, G., Stenborg, G., Vourlidas, A., Freeland, S. and Howard, R. (2009): The SOHO/LASCO CME Catalog, Earth, Moon and Planets, 104, 295313.

[41] Gosling, J.T., Hildner, E., MacQueen, R.M., Munro, R.H., Poland, A.I. and Ross, C.L. (1974): Mass ejections from the Sun: A view from Skylab, Journal of Geophysical Research, 79, 45814587.

[42] Hale, G.E., Ellerman, F., Nicholson, S.B. and Joy, A.H. (1919): The magnetic polarity of sun-spots, The Astrophysical Journal, 49 153-186.

[43] Harrison, R.A. (1995): The nature of solar flares associated with coronal mass ejection, Astronomy \& Astrophysics, 304, 585-594.

[44] Hathaway, D.H. (2010): The Solar Cycle, Living Reviews Solar Physics, 7, 1. URL http: //www. livingreviews.org/lrsp-2010-1 (cited on 11.07.2016).

[45] Hirayama, T. (1974): Theoretical Model of Flares and Prominences / I: Evaporating Flare Model, Solar Physics, 34, 323-338.

[46] Hood, A.W. and Priest, E.R. (1979): Kink instability of solar coronal loops as the cause of solar flares, Solar Physics 64, 303-321.

[47] House, L.L., Wagner, W.J., Hildner, E., Sawyer, C. and Schmidt, H.U. (1981): Studies of the corona with the Solar Maximum Mission coronagraph/polarimeter, The Astrophysical Journal, 244, L117-L121.

[48] Howard, R.A., Sheeley, N.R., Koomen, M.J. and Michels, D.J. (1985): Coronal Mass Ejections: 1979 - 1981, Journal of Geophysical Research, 90, 8173-8191. 
[49] Howard, R.A., Plunkett, S. and Rich, N. (2004): Data Management Plan for the Sun Earth Connection Coronal and Heliospheric Investigation (SECCHI), Naval Research Laboratory, E.O. Hulburt Center for Space Research, http://secchi.nrl.navy.mil/dps/Data_ Management_Plan_30June04.pdf.

[50] Howard, R.A. (2006): A Historical Perspective on Coronal Mass Ejections, Geophysical Monograph Series, 165, 7-13.

[51] Howard, R.A., Moses, J.D., Vourlidas, A., Newmark, J.S., Socker, D.G., Plunkett, S.P., Korendyke, C.M., Cook, J.W., Hurley, A., Davila, J.M., Thompson, W.T., St Cyr, O.C., Mentzell, E., Mehalick, K., Lemen, J.R., Wuelser, J.P., Duncan, D.W., Tarbell, T.D., Wolfson, C.J., Moore, A., Harrison, R.A., Waltham, N.R., Lang, J., Davis, C.J., Eyles, C.J., Mapson-Menard, H., Simnett, G.M., Halain, J.P., Defise, J.M., Mazy, E., Rochus, P., Mercier, R., Ravet, M.F., Delmotte, F., Auchere, F., Delaboudiniere, J.P., Bothmer, V., Deutsch, W., Wang, D., Rich, N., Cooper, S., Stephens, V., Maahs, G., Baugh, R., McMullin, D. and Carter, T. (2008): Sun Earth Connection Coronal and Heliospheric Investigation (SECCHI), Space Science Reviews, 136, 67-115.

[52] Howard. T. (2011): Coronal Mass Ejections: An Introduction, Astrophysics and Space Science Library, Springer, New York.

[53] Hudson, H.S., Lemen, J.R., St. Cyr, O.C., Sterling, A.C. and Webb, D.F. (1998): X-Ray coronal changes during halo CMEs, Geophysical Research Letters, 25, 2481-2484.

[54] Hundhausen, A.J., Sawyer, C.B., House, L., Illing, R.M.E. and Wagner, W..J. (1984): Coronal mass ejections observed during the Solar Maximum Mission: Latitude distribution and rate of occurrence, Journal of Geophysical Research: Space Physics, 89, 2639-2646.

[55] Illing, R.M.E. and Hundhausen, A.J. (1985): Observation of a coronal transient from 1.2 to 6 solar radii, Journal of Geophysical Research, 90, 275-282.

[56] Inhester, B. (2006): Stereoscopy basics for the STEREO mission, arxiv.org, http://arxiv . org/abs/astro-ph/0612649v1, ADS Bibliographic Code 2006astro.ph.12649I.

[57] Kahler, S.W. and Webb, D.F. (2007): V arc interplanetary coronal mass ejections observed with the Solar Mass Ejection Imager, Journal of Geophysical Research, 112, 9103.

[58] Kaiser,M.L., Kucera,T.A., Davila,J.M., St. Cyr,O.C., Guhathakurta,M. and Christian,E. (2008): The STEREO mission: An introduction, Space Science Reviews, 136, 5-16.

[59] Kivelson, M.G. and Russell, C.T. (1995): Introduction to Space Physics, Cambridge University Press.

[60] Krall, J. and St. Cyr, O.C. (2006): Flux-rope Coronal Mass Ejection geometry and its relation to observed morphology, The Astrophysical Journal, 652, 1740-1746.

[61] Kuperus, M. and Raadu, M.A. (1974): The Support of Prominences Formed in Neutral Sheets, Astrononmy and Astrophysics, 31, 189.

[62] Lawrence, J.D. (1972): A Catalog of Special Plane Curves, Dover Publications, Mineola, N.Y., pp. 4-5, 121-123, 145, 151, 184.

[63] Liewer, P.C., Dejong, E.M., Hall, J.R., Braswell, S.J., Thompson, W.T. and Howard, R. (2007): Stereoscopic analysis of CME-related coronal activity using STEREO/SECCHI observations, American Geophysical Union, Fall Meeting 200\%, Abstract \#SH41B-02.

[64] Liu, Y., Davies, J.A., Luhmann, J.G., Vourlidas, A., Bale, S.D. and Lin, R.P. (2010): Geometric triangulation of imaging observations to track coronal mass ejections continuously out to 1 AU, The Astrophysical Journal Letters, 710, 82-87. 
[65] Low, B.C. and Hundhausen, J.R. (1995): Magnetostatic structures of the solar corona. II. The magnetic topology of quiescent prominences, The Astrophysical Journal, 443, 818-836.

[66] Low, B.C. (2001): Coronal mass ejections, magnetic flux ropes, and solar magnetism, Journal of Geophysical Research, 106, 25141-25163.

[67] Lugaz, N. (2010): Accuracy and limitations of fitting and stereoscopic methods to determine the direction of coronal mass ejections from heliospheric imagers observations, Solar Physics, 267, 411-429.

[68] Luhmann, J.G., Curtis, D.W., Schroeder, P., McCauley, J., Lin, R.P., Larson, D.E., Bale, S.D., Sauvaud,J.-A., Aoustin, C., Mewaldt, R.A., Cummings, A.C., Stone, E.C., Davis, A.J., Cook, W.R., Kecman, B., Wiedenbeck, M.E., von Rosenvinge, T., Acuna, M.H., Reichenthal, L.S., Shuman, S., Wortman, K.A., Reames, D.V., Mueller-Mellin, R., Kunow, H., Mason, G.M., Walpole, P., Korth, A., Sanderson, T.R., Russell, C.T. and Gosling, J.T. (2007): STEREO IMPACT Investigation Goals, Measurements, and Data Products Overview, Space Science Reviews, 136, 117-184.

[69] Martin, S.F. (1986): Recent observations of the formation of filaments, Coronal and Prominence Plasmas, ed. A. Poland, pp.73-80, NASA CP-2422.

[70] McClintock, B.H. and Norton, A.A. (2013): Recovering Joy's Law as a Function of Solar Cycle, Hemisphere, and Longitude, Solar Physics, 287, 215-227.

[71] Michels, D.J., Howard, R.A., Koomen, M.J. and Sheeley, Jr.,N.R. (1980): Satellite observations of the outer corona near sunspot maximum, Radio Physics of the Sun, 439.

[72] Mierla, M., Inhester, B., Antunes, A., Boursier, Y., Byrne, J.P., Colaninno, R., Davila, J., de Koning, C.A., Gallagher, P.T., Gissot, S., Howard, R.A., Howard, T.A., Kramar, M., Lamy, P., Liewer, P.C., Maloney, S., Marqu, C., McAteer, R.T.J., Moran, T., Rodriguez, L., Srivastava, N., St. Cyr, O.C., Stenborg, G., Temmer, M., Thernisien, A., Vourlidas, A., West, M.J., Wood, B.E. and Zhukov, A.N. (2010): On the 3-D reconstruction of coronal mass ejections using coronagraph data, Annales Geophysicae, 28, 203-215.

[73] Millward, G., Biesecker, D., Pizzo, V. and de Koning, C.A. (2013): An operational software tool for the analysis of coronagraph images: Determining CME parameters for input into the WSA-ENLIL heliospheric model, Space Weather, 11, 57-68.

[74] Millward, G. and Steenburgh, R. (2013): Instructions on installing and using the SWPC CME Analysis Tool (CAT), Software Manual published at NOAA/SWPC, ftp://198.118. 248.137/solarsoft/packages/swpc_cat/doc/SWPC_CAT_README.pdf.

[75] Mitchell, W.M. (1916): The history of the discovery of the solar spots, Popular Astronomy, $\mathbf{2 4}, 82$.

[76] Munro, R.H., Gosling, J.T., Hildner, E., MacQueen, R.M., Poland, A.I. and Ross, C.L.: 1979, The association of coronal mass ejection transients with other forms of solar activity, Sol. Phys., 45377.

[77] Moore, R.L. and LaBonte, B.J. (1980): The filament eruption in the 3B flare of July 29, 1973: Onset and magnetic field configuration, Solar and Interplanetary Dynamics, IAU Symposia, 91, 207-210.

[78] Moore, R.L., Sterling, A.C., Hudson, H.S. and Lemen, J.R. (2001): Onset of the Magnetic Explosion in Solar Flares and Coronal Mass Ejections, The Astrophysical Journal, 552, 833848.

[79] Moran, T.G., Davila, J.M. and Thompson, W.T. (2010): Three-dimensional polarimetric coronal mass ejection localization tested through triangulation, The Astrophysical Journal, $\mathbf{7 1 2}, 453-458$. 
[80] Mouschovias, T.C. and Poland, A.I. (1978): Expansion and broadening of coronal loop transients - a theoretical explanation, The Astrophysical Journal, 220, 675-682.

[81] Munro, R.H., Gosling, J.T., Hildner, E., MacQueen, R.M., Poland, A.I. and Ross, C.L. (1979): The association of coronal mass ejection transients with other forms of solar activity, Solar Physics, 61, 201-215.

[82] Neupert, W.M. (1988): A search for the origins of a possible coronal mass ejection in the low corona, Solar and stellar coronal structure and dynamics; Proceedings of the Ninth Sacramento Peak Summer Symposium, 528-539.

[83] National Oceanic and Atmospheric Administration, Space Weather Prediction Center (2012): Users Guide to the Preliminary Report and Forecast of Solar Geophysical Data, www.swpc. noaa.gov/weekly/Usr_guide.pdf.

[84] Odstrcil, D. (2003): Modeling 3-D solar wind structure, Advances in Space Research, 32 (4), $497-506$

[85] Parenti, S. (2014): Solar Prominences: Observations, Living Reviews Solar Physics, 11, 1. URL http://dx.doi.org/10.12942/Irsp-2014-1 (cited on 16.04.2016).

[86] Parker, E.N. (1958): Dynamics of the interplanetary gas and magnetic fields, The Astrophysical Journal, 128, 664-676.

[87] Reames, D.V. (1999): Particle acceleration at the Sun and in the heliosphere, Space Science Reviews, 90, 413-491.

[88] Robbrecht, E., Patsourakos, S. and Vourlidas, A. (2009): No Trace left behind: STEREO Observation of a Coronal Mass Ejection without low-coronal signatures, The Astrophysical Journal, 701, 283-291.

[89] Rouillard, A.P., Davies, J.A., Forsyth, R.J., Rees, A., Davis, C.J., Harrison, R.A., Lockwood, M., Bewsher, D., Crothers, S.R., Eyles, C.J., Hapgood, M. and Perry, C.H. (2008): First imaging of corotating interaction regions using the STEREO spacecraft, Geophysical Research Letters, 35, L10110.

[90] Rust, D.M. and Webb, D.F. (1977): Soft X-Ray observations of large-scale coronal active region brightenings, Solar Physics, 54, 403-417.

[91] Rust, D.M. and Kumar, A. (1994): Helical magnetic fields in filaments, Solar Physics, 155, 69-97.

[92] Savani, N., Owens, M.J., Rouillard, A.P., Forsyth, R.J. and Davies, J.A. (2010): Observational evidence of a coronal mass ejection distortion directly attributable to a structured solar wind, The Astrophysical Journal Letters, 714, 128-132.

[93] Scherrer, P.H., Bogart, R.S., Bush, R.I., Hoeksema, J.T., Kosovichev, A.G., Schou, J., Rosenberg, W., Springer, L., Tarbell, T.D., Title, A., Wolfson, C.J. and Zayer, I. (1995): The Solar Oscillations Investigation - Michelson Doppler Imager, Solar Physics, 162, 129-188.

[94] Schwenn, R. (2006): Space Weather: The Solar Perspective, Living Reviews Solar Physics, 3, 2. URL http://www.livingreviews.org/lrsp-2006-2 (cited on 16.12.2015).

[95] Sheeley, Jr.,N.R., Howard, R.A., Michels, D.J. and Koomen, M.J. (1980): Solar observations with a new earth-orbiting coronagraph, Solar and Interplanetary Dynamics, 91, 55-59.

[96] Sheeley, Jr.,N.R., Howard, R.A., Koomen, M.J., Michels, D.J., Schwenn, R., Mühlhäuser, K.H. and Rosenbauer, H. (1985): Coronal mass ejections and interplanetary shocks, Journal of Geophysical Research, 90, 163-175. 
[97] Sheeley, Jr.,N.R., Wang, Y.-M., Hawley, S.H., Brueckner, G.E., Dere, K.P., Howard, R.A., Koomen, M.J., Korendyke, C.M., Michels, D.J., Paswaters, S.E., Socker, D.G., St. Cyr, O.C. and Wang, D. (1997): Measurements of flow speeds in the corona between 2 and $30 \mathrm{r}_{\odot}$, The Astrophysical Journal, 484, 472-478.

[98] Sheeley, N.R., Walters, J.H., Wang, Y.-M. and Howard, R.A. (1999): Continuous tracking of coronal outflows: Two kinds of coronal mass ejections, Journal of Geophysical Research, 104, $24739-24767$.

[99] Sheeley, N.R., Herbst, A.D., Palatchi, C.A., Wang, Y.-M., Howard, R.A., Moses, J.D., Vourlidas, A., Newmark, J.S., Socker, D.G., Plunkett, S.P., Korendyke, C.M., Burlaga, L.F., Davila, J.M., Thompson, W.T., St Cyr, O.C., Harrison, R.A., Davis, C.J., Eyles, C.J., Halain, J.P., Wang, D., Rich, N.B., Battams, K., Esfandiari, E. and Stenborg, G. (2008): Heliospheric images of the solar wind at Earth, The Astrophysical Journal, 675, 853-862.

[100] Shi, T., Wang, Y., Wan, L., Cheng, X., Ding, M. and Zhang, J. (2015): Predicting the arrival time of coronal mass ejections with the graduated cylindrical shell and drag force model, The Astrophysical Journal, 806, 271-279.

[101] Sterling, A.C., Hudson, H.S., Thompson, B.J. and Zarro, D.M. (2000): Yohkoh SXT and SOHO EIT observations of sigmoid-to-arcade evolution of structures associated with halo coronal mass ejections, The Astrophysical Journal, 532, 628-647.

[102] Stix, M. (2002): The Sun - An Introduction, Springer, (2nd Edition).

[103] Subramanian, P. and Dere, K.P. (2001): Source Regions of Coronal Mass Ejections, The Astrophysical Journal, 561, 372-395.

[104] Svestka, Z., Farnik, F., Hudson, H.S. and Hick, P. (1998): Large-scale active coronal phenomena in Yohkoh SXT images, Solar Physics, 182, 179-193.

[105] Thernisien, A.F.R., Patel, N.S., Howard, R.A., Marque, C. and Vourlidas, A. (2004): Raytracing Software for the Simulation of the Solar K-Corona, American Geophysical Union, Fall Meeting 2004, Abstract \#SH21B-0404.

[106] Thernisien, A.F.R., Howard, R.A. and Vourlidas, A. (2006): Modeling of Flux Rope Coronal Mass Ejections, The Astrophysical Journal, 652, 763-773.

[107] Thernisien, A.F.R., Vourlidas, A. and Howard, R.A. (2009): Forward Modeling of Coronal Mass Ejections Using STEREO/SECCHI Data, Solar Physics, 256, 111-130.

[108] Thernisien, A.F.R. (2011): Implementation of the Graduated Cylindrical Shell Model for the three-dimensional reconstruction of Coronal Mass Ejections, The Astrophysical Journal Supplement Series, 194, 33.

[109] Thernisien, A.F.R., Vourlidas, A. and Howard, R.A. (2011): CME reconstruction: PreSTEREO and STEREO era, Journal of Atmospheric and Solar-Terrestrial Physics, 73, 11561165.

[110] Thompson, W. (2004): STEREO Science Operations Plan, STEREO Science Center, NASA, http://stereo-ssc.nascom.nasa.gov/publications/stereo_sop.pdf.

[111] Török, T. and Kliem, B. (2003): The evolution of twisting coronal magnetic flux tubes, Astronomy \& Astrophysics, 406, 1043-1059.

[112] Tripathi, D., Bothmer, V. and Cremades, H. (2004): The basic characteristics of EUV posteruptive arcades and their role as tracers of coronal mass ejection source regions, Astronomy \& Astrophysics, 422, 337-349.

[113] Van Ballegooijen, A. and Martens, P. (1989): Formation and eruption of solar prominences, The Astrophysical Journal, 343, 971-84. 
[114] Vourlidas, A., Subramanian, P., Dere, K.P. and Howard, R.A. (2000): Large-Angle Spectrometric Coronagraph measurements of the energetics of Coronal Mass Ejections, The Astrophysical Journal, 534, 456-467.

[115] Vourlidas, A., Wu, S.T., Wang, A.H., Subramanian, P. and Howard, R.A. (2003): Direct detection of a coronal mass ejection-associated shock in large angle and spectrometric coronagraph experiment white-light images, The Astrophysical Journal, 598, 1392-1402.

[116] Vourlidas, A. and Ontiveros, V. (2009): A review of coronagraphic observations of shocks driven by coronal mass ejections, arxiv.org, http://arxiv.org/abs/0908.1996v1.

[117] Vourlidas, A., Colaninno, R., Nieves-Chinchilla, T. and Stenborg, G. (2011): The first observation of a rapidly rotating coronal mass ejection in the middle corona, The Astrophysical Journal Letters, 733, L23.

[118] Vourlidas, A., Lynch, B.J., Howard, R.A. and Li, Y. (2013): How many CMEs have flux ropes? Deciphering the signatures of shocks, flux ropes, and prominences in coronagraph observations of CMEs, Solar Physics, 284, 179-201.

[119] Wagner, W.J. (1984): Coronal mass ejections, Annual Review of Astronomy $\mathscr{G}$ Astrophysics, 22, 267-289.

[120] Webb, D.F. and Hundhausen, A.J. (1987): Activity associated with the solar origin of coronal mass ejections, Solar Physics, 108, 383-401.

[121] Xie, H., Ofman, L. and Lawrence, G. (2004): Cone model for halo CMEs: Application to space weather forecasting, Journal of Geophysical Research, 109, A03109.

[122] Xue, X.H., Wang, C.B. and Dou, X.K. (2005): An ice-cream cone model for coronal mass ejections, Journal of Geophysical Research, 110, A08103.

[123] Yan, Y., Deng, Y., KarlickÂA'y, M., Fu, Q., Wang, S. and Liu, Y. (2001): The Magnetic Rope Structure and Associated Energetic Processes in the 2000 July 14 Solar Flare, The Astrophysical Journal Letters, 551, L115-L119.

[124] Yashiro, S., Gopalswamy, N., Michalek, G., St. Cyr, O.C., Plunkett, S.P., Rich, N.B. and Howard, R.A. (2004): A catalog of white light coronal mass ejections observed by the SOHO spacecraft, Journal of Geophysical Research, 109, (A07105).

[125] Yurchyshyn, V., Yashiro, S., Abramenko, V., Wang, H. and Gopalswamy, N. (2005): Statistical distributions of speeds of coronal mass ejections, The Astrophysical Journal, 619, 599-603.

[126] Zhao, X., Plunkett, S.P. and Lui, W. (2002): Determination of geometrical and kinematical properties of halo coronal mass ejections using the cone model, Journal of Geophysical Research, 107 (A8), 1223. 


\section{Acknowledgements}

First I would like to thank my supervisor Volker Bothmer for his scientific support, advice and funding of my position. The work on several projects allowed me to participate in conferences and to meet international colleagues. Furthermore I thank Ansgar Reiners and Stefan Dreizler who gave support as additional members of my advisory committee. Special thanks go to Ansgar Reiners and Jörg Büchner who reviewed my thesis as referees.

It was very enriching over the past years to work together with my office mates. I had inspiring and motivating discussions with Malte Venzmer, Adam Pluta and Jonas Hesemann about solar physics, Star Wars and Star Trek. Thanks for the pleasant time also outside the office. I want to thank Niclas Mrotzek, Johannes Hinrichs, Jens Rodmann and Laura Volpes for the good collaboration in our space weather working group and our joint experiences on business trips.

It was very valuable for me to meet new and inspiring colleagues on international business trips and to see their research institutes in Bruessel, Washington, Boulder and Graz ;). At this point thanks are due to Manuela Temmer, Angelos Vourlidas, Arnauld Thernisien and Rodney Viereck. They made it possible to work together on papers and to visit the Naval Research Laboratory (Washington D.C.) and the Space Weather Prediction Center at Boulder, Colorado. This would not be possible without the funding from FP7 projects organised by Volker Bothmer.

The CME online database, which was introduced within the AFFECTS project, was developed by Sabyasachi Gosh and Julius Achenbach. I thank both for the great work. My colleagues Malte Venzmer, Jens Rodmann and Ulf Seemann gave support with proof-reading my thesis. Special thanks to them! Finally I want to thank my family, dojo and friends for their encouragement. May the force be with you.

The research leading to these results has received funding from the DLR project Stereo/Corona and the European Community's Seventh Framework Programme (FP7) under the grant agreement of projects SOTERIA, AFFECTS, eHeroes and HELCATS. 



\section{Declaration}

I hereby declare that the thesis submitted is my own unaided work. All direct or indirect sources used are acknowledged as references. This paper was not previously submitted to another examination board and has not been published.

Göttingen,

Eckhard Bosman 



\title{
Curriculum Vitae
}

\author{
Dipl.-Phys. $\quad$ Eckhard Bosman \\ Geburtstag: $\quad 13.11 .1980$ \\ Geburtsort: Köln \\ Staatsangehörigkeit: Deutsch
}

\begin{tabular}{ll}
\hline $10 / 2014-12 / 2016$ & Promotionsstudent, Dissertation \\
$05 / 2014-09 / 2014$ & HELCATS, EU FP7 project \\
$03 / 2014-04 / 2014$ & eHeroes, EU FP7 project \\
$01 / 2013-02 / 2014$ & AFFECTS, EU FP7 project \\
$01 / 2012-12 / 2012$ & Stereo/Corona, DLR \\
$11 / 2009-12 / 2011$ & SOTERIA, EU FP7 project \\
$2009-2014$ & wissenschaftlicher Mitarbeiter und Promotionsstudent \\
& am Institut für Astrophysik, Georg-August-Universität \\
& Göttingen \\
$2001-2008$ & Studium der Physik, Georg-August-Universität Göttingen \\
& Abschluss: Diplom-Physiker \\
$2000-2001$ & Wehrdienst \\
$1993-2000$ & Gymnasium Alfeld (Leine) \\
& Abschluss: Allgemeine Hochschulreife
\end{tabular}

